\title{
DSCLAMIN
}

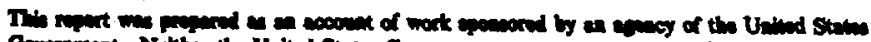

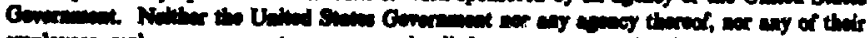

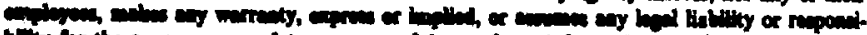

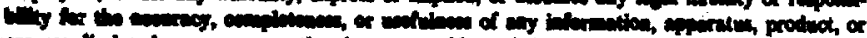

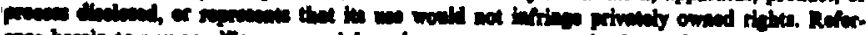

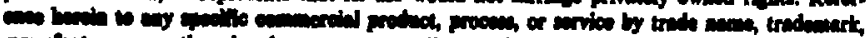

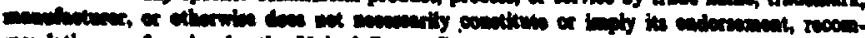

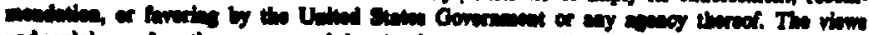

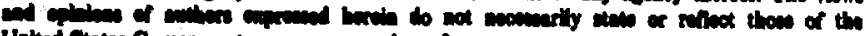

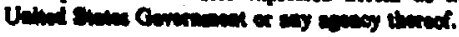

\section{Fragmentation of Suddenly Heated Liquids}

James Allen Blink

(Doctor of Philosophy)

Manuscript date: March 1985

\section{LAWRENCE LIVERMORE LABORATORY \\ University of California - Livermore, California $\bullet 94550$}

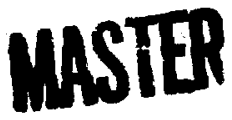

A vailable from: National Technica! Information Service - U.S. Department of Commerce 52as Port Royal Road - Springfietd, VA 22161 -\$20.00 per copy - (Microliche \$4.50) 


\section{FRAGMENTATION OF SUODEMLY HEATED LIQUIDS}

\section{By}

\section{JAMES NLEN BLINK \\ M.S. (Georgia Institute of Technology) 1971}

\section{DISSERTATIO}

Submitted in partial satisfaction of the requirements for the degree of DOCTOR OF PHILOSOPHY

in

Engineering

in the

GRADUATE DIYISION

of the

UNIVERSITY OF CALIFORNIA

DAYIS

\section{Approved:}

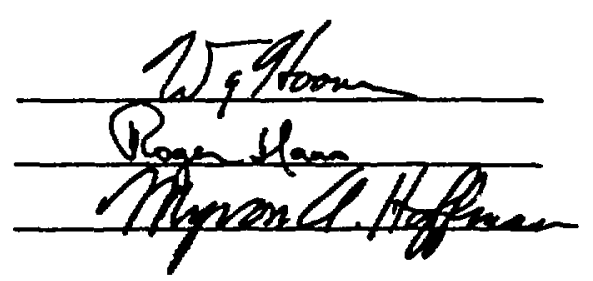

Comittee in Charge 
Abstract

Fragmentation of free liquids in Inertial confinement Fusion reactors could determine the upper bound on reactor pulse rate. The $x$-ray ablated materials must cool and recondense to allow driver beam propagation. The increased surface area caused by fragmentation w/ll enthence the cooling and condensation rates.

Relaxation from the suddenly heated state will move a liquid into the negative pressure region under the liquid-vapor P-V dome. The IIthiun equation of state was used to demonstrate that neutron-induced vaporization uses only a minor fraction of the added heat, much less thall would be required to drive the expansion. A $77 x$ expansion of the lithlim is required before the rapid vaporization process of spinodal decomposition could begin, and nucleation and growth are too slow to contribute to the expansion.

An energy minimization procedure determined the fragment slze that produces the least amount of non-fragment-center-of-mass energy, i.e., the sum of the surface and dilational kinetic energies. Considerable fragmentation is predicted in the high energy density HYLIFE reactor, with minimum fragmentation in the low energy density Pulse*Star, EAGL, and HIBALL reactors.

A two-dimensional molecular dynamics (MD) code was developed to test the model at a microscopic scale for the Lennard-Jones (LJ) fluid. The two LJ constains, and $\varepsilon$, were chosen to represent lithium. The LJ properties were generated from equatlon of state data 
and UD calculations. A 2-D calculation most closely models a jet, permits easy graphical inspection of the results, end allows consideration of lerger radius systems. (Surface tension is a strong function of the system radius at this scale.) MD calculations were run using five levels of heating and four system sizes. The numerical experiments were consistent with the model: fragment size (In atoms) $\sim$ (system slze) $^{2 / 3}$ and (initlal pressure) $)^{-20 / 3}$. The larger fragment sizes were within en order of manitude of the predictions. Additional analysis of two Italien three dimensional Mo experiments also agreed with the model. 
$-1 x-X-$

Dedication

To ny wife, Frolida Del Papa Blink, without whose love and encouregment this dissertation would not have been completed. 


\section{Acknowledgments}

Particular thenks go to ny wife, Freida, who encouraged this work and helped ebsorb the pressures it genarated. Davien Millard and Loulse Harlen deserve spocial thanks for their tireless work lij typing much of the final manuscript. In addition, Karen Hogua, Susen Belghley, Nancy Barnes, and Audrey DeRodeff typed portlons of the first draft.

John Emmett, Lewrence LIvermore National Leboratory's Assoclate Director for Lasers, encouraged the to begin the PhD program, and John Holzrichter, his Deputy for Inertial Confinenent Fusion, urged me to finish In a timely fashion. MIke Monsler and Bill Hogan, my direct supervisors during this study, were instrumental in providing the tine and computer resources needed in the study. Wy Energy and Military Applications Group colleagues, Wayne Meler, John Pitts, George Lasche, and in particular, Jack Hovingh, were excellent sounding boards for my preliminary results.

Hy thesis comnittee, Mike Hoffman, Roger Haas, and particularly the chalman, Bill Hoover, devoted many hours to inspire and review wy work. Invariably, Bill's casual observations and suggestlons would prove true -- after I spent several weeks studying and running computer simulations of then. These suggestions tended to focus my work without leading me step-by-step, and I will always be grateful to Bill for using this wonderful teaching technique.

Thanks are also du to several groups of people: Pete Keller, 
Kelly O'Halr, Giles Peterson, Bill warren, and Jeff Rowe (all of LLM) wo helped modernize my computation models and computer grephics; John Blunden of Luk who made a movie of one of my computer simulations; Glorla Martinez of LLML tho quickly obtalned needed references; Dave Young and Lew Glem of LLN and John Barker and Farid Abrchen of IBN Lebs who offered noleculer dynemics and equation of state advice; $G$. Jacuccl, Y. Pancharipande and A. Vicentini of the University of Ililnols wo provided coples of two of their molecular dynemlcs calculations; Ollle Parker and Ullyses Falls of LLML who supervised preparation of my Lllustrations; and the Veteren's Adninistration which providod financial support under the GI BIll. Special thenks also go to Nate Hof fman of ETEC who kept reminding we that this dissertation slobject is of vital importance to Inertial Confinement Fusion reactor design. 
Fragmentation of Suddenly Hoated Liquids

by James A. Blink

Teble of Contents

Choter

Pan No.

l. Introduction $\ldots \ldots \ldots \ldots \ldots \ldots \ldots \ldots \ldots \ldots \ldots \ldots \ldots \ldots, 1$

2. Importance of Liquid fragmentation to Inartial ....... 5 Fusion Reactor Desion

3. Liquid Fragmentation in Atomizer Nozzles ........... 19

4. Rapid Vaporization as a Fragmentation Machanism $\ldots . . .21$ 4.1. Lithium Phase Diagram $\ldots \ldots \ldots \ldots \ldots \ldots \ldots \ldots . . \ldots 23$

4.2. Two-Dinensional Lennard-Joneslum Phase Diagran . 33

4.3. Thermodynamic Paths Followed on the P-V

Dlagrem and After Isochorlc Hating ......... 56

4.4. Spinodal Decomposition .................. 62

4.5. Nucleation and Growth ................... 68

4.6. Evaluation of the Raplos: porlzation Approsch to Liquid fragmentation $\ldots \ldots \ldots \ldots \ldots \ldots \ldots \ldots . . \ldots 9$ 
5. Force and Energy Balance Models for Fragmentation .... 81

5.1. Balancing the Isochoric-Heating Pressure by

Surface Tension $\ldots \ldots \ldots \ldots \ldots \ldots \ldots \ldots \ldots \ldots . . .63$

5.2. Balancing the Post-Relaxation Dynamic Pressure

by Surface Tension $\ldots \ldots \ldots \ldots \ldots \ldots \ldots \ldots \ldots, 84$

5.3. Minimizing the Sum of the Surface Energy and

the Comoving Dilational Kinetic Energy (The

Grady-Glenn Model) $\quad \ldots \ldots \ldots \ldots \ldots \ldots \ldots \ldots \ldots \ldots$

5.3.1. Model Derivation in Two and Three

Dimensions $\ldots \ldots \ldots \ldots \ldots \ldots \ldots \ldots \ldots, 86$

5.3.2. Effect of Considering the Velocity

Distribution Rather Than the Average

Disassembly Velocity in Model $3 . . \ldots . .99$

5.3.3. Application to Several ICF Reactors .... 100

5.3.4. Application to Small Lennard-Jonesium

Systems $\ldots \ldots \ldots \ldots \ldots \ldots \ldots \ldots \ldots \ldots . \ldots 103$

5.4. Evaluation of the Force and Energy Balance

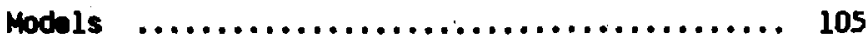

6. Molecular Dymemics Experiment Dovelopment .......... 107

6.1. Prevlous Molecular Dynamics Modeling of Liquids . 108

6.2. Solving the Equations of Motion ............ 111

6.3. The Unsuitebility of the Parabolic Potential ... 116

6.4. The Lennard-Jones (6-12) Potentlal .......... 125 
Cheoter

6.5. Establishing the Initial Density Starting with

a Zero Temperature Solid .................. 135

6.6. Tenperature Modlfication and Isochoric Heat

Addition $\ldots \ldots \ldots \ldots \ldots \ldots \ldots \ldots \ldots \ldots \ldots \ldots, 141$

6.7. Graphlc Output $\ldots \ldots \ldots \ldots \ldots \ldots \ldots \ldots \ldots \ldots \ldots . \ldots \ldots$

6.8. Tabular Output $\ldots \ldots \ldots \ldots \ldots \ldots \ldots \ldots \ldots \ldots \ldots \ldots, 157$

6.8.1. Data Summrized Over All Atoms ....... 158

6.8.2. Data Sumarized By Cluster .......... 160

6.9. Computational Efficiency $\ldots \ldots \ldots \ldots \ldots \ldots \ldots \ldots, 163$

7. Two-Dimensional Molecular Dynamic Experiment

Results $\ldots \ldots \ldots \ldots \ldots \ldots \ldots \ldots \ldots \ldots \ldots \ldots \ldots \ldots, 171$

7.1. Partition of Isochoric Heating Energy Between

Kinetlc and Potentlal Energles $\ldots \ldots \ldots \ldots \ldots \ldots, 172$

7.2 Material Propertles $\ldots \ldots \ldots \ldots \ldots \ldots \ldots \ldots \ldots, 175$

7.2.1. The Gronelsen Parameter $\ldots \ldots \ldots \ldots \ldots 176$

7.2.2. Wave Speed $\ldots \ldots \ldots \ldots \ldots \ldots \ldots \ldots \ldots \ldots, 186$

7.2.3. Surface Tension $\ldots \ldots \ldots \ldots \ldots \ldots \ldots \ldots . . . . .196$

7.3. Holecular Dynamics Experiment Results ........ 218

7.3.1. Two Dinensional Molecular Dynenics

Resuits $\ldots \ldots \ldots \ldots \ldots \ldots \ldots \ldots \ldots \ldots \ldots .219$

7.3.2. Three Dimensional Molecular Dynamics

Results $\ldots \ldots \ldots \ldots \ldots \ldots \ldots \ldots \ldots \ldots . \ldots \ldots$ 
Choter

Pen No.

7.4. Comparison of the Molecular Dynmics Results

to the Models Derived in Chapter Five ........ 245

7.4.1. Comparison of the Two Dimensional

Molecular Dynemics Rasults to the

Hydrodymanlc Modols ................ 249

7.4.2. Comparison of the Three Dimenstonal

Molecular Dymemics Results to Modal 3 .. 268

7.5. Vepor Pressure Formation as a Compating

Phenomenon $\ldots \ldots \ldots \ldots \ldots \ldots \ldots \ldots \ldots \ldots \ldots \ldots \ldots \ldots . \ldots \ldots 2$

8. Conclusions and Recommendations for Further Research ... 276

Reforences

281

Nomenclature

Appendix A. Executlive Summary 297 


\section{FICUnes}

Fioure

Itt.ls

F1g. 2.1. The Lill HIIIE resctor concept. ............. 7

F1g. 2.2. iins of interest for the $1.5 \mathrm{~Hz}$ H.IFE reactor. . 9

Flg. 2.3. The WIsconsin HIENLL roactor concept. ........... 10

FIg. 2.4. The LMK Modifled watted will reactor concept. ... 11

Fig. 2.5. The Buchtel Eace recetor concept. ............. .12

F1y. 2.6. The LUK Pulse*Star reactor concept. ,.......... 14

F1g. 2.7. Combined neutron and gemmerapesition in the HMLFE Ilthium jet array as a function of time. .. 15

F1g. 2.8. Nestron and gemma energy deposition profile in the HMLIFE IIthium jat array.

Fig. 4.1. Soft-sphere fit (smooth curves) to axperimental Lithlum tharmodinamic data (points). .......... 26

Fig. 4.2. Equilibrium and nonequilibrium 1sotherms on a schomatle P-Y diagren.

Fig. 4.3. Illustration of Maxwell's equal area rule for determining the coexistence curve. 30

Fig. 4.4. P-Y diagren for lithlim. The sixteen 1sotherms ore at $50^{\circ} \mathrm{C}$ intervals.

Fig. 4.5. The Lennard-Jones 6-12 interatomic potontlal and assoclated force function truncated at $I=2.50 \ldots$. 
Fig. 4.6. Phase dizgram for two-dimensional Lennard-Jonesium (density-temperature plane). .............. 36

Flo. 4.7. Phase diagrem for two-dimensional Lennard-Jonesium (temperature-pressure plane).

Fig. 4.8. Compressibility factor contours for 2-D

Lemard-Jonesium.

Fig. 4.9. Pressure 1sotherms from Barker's equation and

Henderson's Monte Carlo data.

Fig. 4.10. Pressure 1sochores from Barker's equation and

Henderson's Monte Carlo data.

Fig. 4.11. Specific heat from Henderson's Monte Carlo

energy date.

Fig. 4.12. Unit cell for a hexagonal-close-packed lattlce. .

Fig. 4.13. Lattice energy per atom for several variations

of the Lennard-Jones 6-12 potential.

Fig. 4.14. P-y diegram of the heating and initlal expansion

of a HMLIFE innar Jat.

Fig. 4.15. P-Y diagram showing isentropic expension to the spinodal line.

Fig. 4.16. In spinodal decomposition, there is no energy barrier to small phase separations. 63

F1g. 4.17. Free body diagrem of a critical nucleus. 69

F10. 4.18. Free energy as a function of system size. 72 
Fig. 4.19. Free eneroy per atom as a function of system size for constent and size-dependent surface tension. .

Fig. 4.20. If no large nuclol are present, high euportieat is possible before bolling begins.

Flg. 4.21. Bubble growth slows with tim.

Fig. 5.1. Geomatry used in the Gredy-Giem energy minimization model.

Fig. 5.2. Grady's model solects a fragment size that minimizes the sum of the surface plus fragment dilational energy densities.

Fig. 5.3. Comparison of Grady's model and laboratory experiments.

Fig. 5.4. Glenn's calculated velocity profile for uniformly heated spheres.

Fig. 5.5. Glenn's calculated velocity divergence $(-p / \rho)$ and analytic approximation.

Fig. 5.6. Fragment size correction factor for the Grady-Glenn model.

Fig. 6.1. For smell fregments, the surfece energy is lower then prodicted by constant $\gamma$, loeding to smiler fregments then expected. 
Fig. 6.2. Velocity distribution function used to randomly generate the initiel velocities. ............ 114

Fig. 6.3. The IIthium parabolic potential energy function and its associated force function.

Fig. 6.4. Initial conditions for the calculations that

tested the molecular dynanics model against

the published phase diegrem. ............... 126

Fig. 6.5. Time evolution of a solid. ................ 127

Fig. 6.6. Tim evolution of as. ga............... 128

Fig. 6.7. Tim ovolution of a liguid. ................ 130

Flg. 6.8. Time evolution of a solid-vapor state. ........ 131

F1g. 6.9. Time evolution of a liquid-vapor state. ........ 132

Fig. 6.10. Tine evolution of a solid-liquid state. ........ 133

Fig. 6.11. Circularization for a 271 atom system. ........ 136

F1g. 6.12. Circularization for a 2611 atom system. ....... 137

Fig. 6.13. Temparature oscillation during the initial 6000 step equilibration (18.6 ps).

Fig. 6.14. Configuration at the end of 6000 equilibration tim steps (18.6 ps).

F10. 6.15. Final configuration after 1000 isothermal time steps ( 4.4 ps) at temperatures of 0.42 and $0.45 \ldots 146$

F10. 6.16. Final configuration after 1000 isothermal time steps ( 4.4 ps) at temperatures of 0.5 and $0.53 \ldots 147$ 
Elore

It.le

Fig. 6.17. Final configuration after 1000 isotharmi the

steps ( 4.4 ps) at a temporature of 0.55 .

148

Fig. 6.18. Configuration of a 721 atom systom equilibrated to a temperature of 1.153 for 3000 t1me steps (11 ps). . 149

Fig, 6.19. Potential eneroy per atcon as a function of tiles

for seven equllibration runs.

Fig. 6.20. Final configuration of 721 atom system that

froze during temperature equllibration.

Fig. 6.21. Step time dependence on list radius for 2611

atom system.

Fig. 6.22. List update interval for a 2611 atom system. . . . 168

Fig. 6.23. List size for a 2611 atom system. ............ 169

Fig. 7.1. Increase in potential and kinetic energies in the $p=0.6$ molecular dymamics runs after the temperature is increased and maintained at a higher value.

Fig. 7.2. Pressures from the nolecular dynanics runs, Bar' as 's theoretical and monte Carlo calculations, and itenderson's Monte Carlo calculstions. 178

Fig. 7.3. Groneisen paremeter from the Monte Carlo date of Henderson and Barker. 
Fionre

Itile

Pane:

Fig. 7.4. Cumulative Grünelsen paraneter defined by the pressure and energy differences between the heated state and the saturated liquid at the $\operatorname{sem}$ density. 1.82

Fig. 7.5. Prassure as a function of time for two constant volume and temperature molecular dynamics calculations.

Fig. 7.6. Configurational plus kinetic energy per aton for two 2611 atom liquids confined by walls. 184

FIg. 7.7. Sound speed computed from Henderson's ${ }^{24}$ Monte Carlo pressure and energy data and from Barker's pressure equation. 188

Fig. 7.8. Density and cumulative density profiles 1.4 ps

after renoval of the wall from a liquid equilibrated at $\mathrm{kT} / \mathrm{c}=0.65$.

Fig. 7.9. Average wave speed for each of the molecular dynemics runs. 192

Fig. 7.10. Wave fronc tracks and particle velocity magnitudes for converging expension and compression waves. .. 194

Fig. 7.11. Cones of particles interacting with a reference atom for a plerrar interfece, large drop, and a smali drop.

Fig. 7.12. Three-dimensional surface tension as a function of tcmperature and drop size. 
Fioure

Title

Parest

F1g. 7.13. Geometry used to calculate $\lambda_{\min }$............. 202

F1g. 7.14. Relationship between the bond and the surface. ... 204

F1g. 7.15. Instanteneous atom positions and time averagod density and pressure profiles. $\ldots \ldots \ldots \ldots \ldots \ldots, 205$

Flg. 7.16. Two dimensional volume element used to derive the relationship between $P_{N}, 2 P_{N} / \partial r$, and $P_{T} \ldots \ldots \ldots \ldots 207$

F1g. 7.17. Hypothatical strip used to determine the force balence that speclfies the surface tension. ...... 208

F10. 7.18. Two dimensional surface tension from molecular dynamics calculations and from scaled three dimensional values. 214

Fig. 7.19. Potential energy per atom as a function of drop size at times long after fragmentation. 217

Fig. 7.20. Initial conditions for the two dimensional molecular dynamics fragmentation calculations. 221

Fig. 7.21. Snapshots of the atonic configurations at four

times during the fragnentation process of a 14491 atom system $(T \sim 1.0)$.

Fig. 7.22. Thermal and translational temperature evolution in the 2-D fragnentation runs.

Fig. 7.23. Thermal temperature evolution of the largest cluster in the 2-D fragmentation runs. ......... 226

Fig. 7.24. Fractional size of the largest cluster in the 2-D fraymentation runs. 
Fig. 7.25. Normallzed size of the largest cluster. 228

F1g. 7.26. Bonds per non-monomer atom in the 2-D fragmentation runs.

F1g. 7.27. Pressure-density paths followed by two runs. .... 231

Fig. 7.28 Fregment size distribution for the 14491 atom run.. 233

F1g. 7.29 Integrated population distributions for three runs. 234 F10. 7.30. Initial temperaturas and densitios for the two

Italien 3-D molecular dymenics fragmentation calculations. 243

F10. 7.31. Evolution of two pressurlzed cubes. ........... 244 F15. 7.32. Ohserved and predicted fragment sizes as a function of initial pressure. 250

Fig. 7.33. Observed and predicted fragment sizes as a function of system size. 251

F1g. 7.34. Ratio of the observed to the predicted fragnent sizes for thirteen 2-D molecular dynamics runs. .. 254 F1g. 7.35. The ratio of the observed to the predicted fragment size, plotted as a function of the observed size... 256

F1g. 7.36. The ratio of the observed to the predicted fregnent size, plotted as a function of the observed size, for the 14491 atom run.

Fig. 7.37. Ratio of the observed to the predicted fregent trenslational temperatures for thirteen 2-0 molecular dymanics runs. 
Fioure

Iitle

Fig. 7.38. The ratio of the observed to the predicted freguent translational temperatures, plotted as a function of the observed size.

Fig. 7.39. The ratio of the observed to the predicted fragnent trenslational temperatures, plotted as a function of the observed size (14491 atoms). 262

Flg. 7.40. Trenslational temperatures scaled to model 3

for the 13 molecular dynemics runs. 265

Fig. 7.4l. The ratio of the observed to the predicted fragment size, plotted as a function of the observed size, for the two 3-D molecular dynamics fragmentation runs.

Fig. 7.42. The ratio of the observed to the predicted fragment translational temperatures, plotted as a function of the observed size, for two 3-D moiecular dynemics fragmentation runs. 271

Fig. 7.43. Mononer population increase and resulting liquid cooling for the lsatl atom run. 273

Fig. A.1. P-Y diagram of the heating and expension of a lithium jet. 302

Fig. A.2. Inllial conditions for the 13 fragmentation runs. . 305 Fig. A.3. Two dimensional surface tension. 309 
Eloure

Fig. A.4. Coserved and predicted fragment sizes as a function of initial pressure.

Fig. A.5, Coserved and predicted fragment sizes as a funclion of system size. 313

Fig. A.6. Snapshots of the atomic conflgurations at four times during the fragmentation process of a 14491 atom system $(T \sim 1.0)$. 315

Fig. A.7. Thermal and translational temperature evolutiun in the 2-D fragmentation runs. 316

Flg. A.8. Normalized size of the largest cluster. 317

Fig. A.9. Pressure-density paths followed by two runs. .... 319

F1g. A.10 Integrated population distributions for three runs. 321 Fig. A.11. Translational temperatures for the 13 runs. 323 
Table 2.1. Fluid properties at $500^{\circ} \mathrm{C} . \quad \ldots \ldots \ldots \ldots \ldots \ldots \ldots, 16$

Table 2.2. Isochoric heating in soverul ICF reactors. $\ldots . \ldots, 18$

Table 4.1. Lithlum data. $, \ldots \ldots \ldots \ldots \ldots \ldots \ldots \ldots \ldots \ldots, \ldots, 27$

Table 4.2. Coeffictents in Barker's pressure equation and resulting pressures at $\rho=0.6 . \ldots \ldots \ldots \ldots \ldots, 37$

Table 4.3. Overlapping points and inconsistencies in the compressibility factor $(P / p k T)$ data. $\ldots \ldots \ldots \ldots \ldots, 40$

Table 4.4. Compressibility factor data. ............... 42

Table 4.5. Equations derived from Barker's pressure equation and Henderson's Monte Carlo data. ............. 49

Table 4.6. SI ve? lies of the reduced units. ................ 53

Table 4.7. Thermodynanic values of the inner HYLIFE jets before and after heating. $\ldots \ldots \ldots \ldots \ldots \ldots \ldots \ldots, 61$

Table 5.1. Predicted fragmentation in several ICF reactors. . 101

Table 6.1. Potential per atom (parabolic potential). ....... 124

Table 6.2. Computational efficiency measures during fragmentation runs on various sized systems. ..... 170 
Table 7.1. Calculation of the sound speed for a density of 0.6 and temperatures of $0.55,0.7$ and $1.0 . \ldots .189$

Table 7.2. Initial conditions for the frapmentation runs. ... 220 Teble 7.3. Constants used in Ree's ${ }^{64}$ pressure equation. .... 240 Table 7.4. Tharmodynamic and geonetric quantitles at the start of the 3-D fragmentation runs.

Table 7.5. Times at which the fragment sizes and trenslatlonal temperatures were compared to the predicted values. 247

Table 7.6. Observed and predicted fragnent fractional sizes for the largest fragment in each 2-D molecular dynamics run.

Teble 7.7. Observed and predicted fragment translational temperatures for the largest fragnent in each 2-D molecular dynamics run. 264

Tuble 7.8. Observed and predicted fragment sizes and trenslational temperatures for the largest fragments in the 3-D molecular dynamics runs.

Teble 7.9. Monomer population and energy for the 13 two dimensional molecular dynemics runs. 275

Teble A.1. Inltial conditions and results from the 2-0 runs. . 306 
Chapter 1: Introduction

In several inertial confinament fusion (ICF) reactor concepts, the fusion neutrons are suddenly deposited into liquid metals having free surfaces within the vacuum chember. The neutron enargy deposition is so fast that the liquid has little opportunity to expend during the deposition time: hence, the deposition is effectively at constent volume, or 1sochoric. In this study, energy deposition is considered "isochoric" If the deposition time is less than the sound wave transit time of the liquid region. The suddenly increased temperature and energy content of the liquid produces a sudden increase in pressure. Since the free surfaces are exposed to effectively zero pressure, tensile rellef waves propagate inward from these free surfaces. Tension eventually causes liquids to cavitate, and this cavitation leads to fragmentation in liquids :ith available expansion volume. The fragments have velocities due to acceleration by the pressure difference across the tensile wave.

Fragmentation of liquid metals inside ICF reactor vessels has two impacts on reactor desion. First, the fragnents eventually collide with the structure, causing a pressure load which can be est Imated from average fragment velocities predicted by continuum mechanics computer codes. (However, fragnent velocity distributions could cause an additional erosion froblem from the higher-then-average-velocity fragments). Second, the fragment size deternines the liquid surface area avalleble to interect thermally with suporheated gas or plasma produced by the absorption of the short-renged fusion $x$ rays and debris. Since the cooling and condensation times depend on the 
cooling surface area, it is possible that the maximum chamber pulse rate will be set by the degree of fregmentation.

The Electric Power Research Institute (EFRI) and the ICF reactor dasion commenity have identified fragmentation as the top priority ICF reactor desion experiment. ${ }^{1}$ Although an EPRI funded report ${ }^{2}$ surveying potential experiments concluded that several energy addition techniques are feasible for lsochorlc heating experiments, the high exporiment costs w1ll cause delays. Further, each technique has flews, including competing physical effects, difficult diagnostics, or Insufficient energy for full-scale simulations. This study develops the models needed for experiment design and valldates them at the microscopic level using molecular tynamics computer simulations. The results of this study should Increase the credibllity of ICF reactor desions, prepare the way for laboratory (macroscopic) simulations of liquid fragmentation, and ald in the desion of an ICF Engineering Test Reactor in which full scale testing of the models will occur.

Liquid fragmentation was approached fron two viowpoints before this study began. In the plrst, the expansion of the fluid into the non-equilibrium reglin under the ilquid-vapor two-phase dome was considored to be resolved by rapid phase separation back to the saturation lines: Further, the phise change was considered the source of the energy driving the expansion and conseqcent vaporization. The secend viempoint wa purely hydrodynemic. Although the expension was ales eseannd to proceed into the tenslle region (negative pressure) une the liquid-vepor tio-phase dome, no 1madiate phase separation we comisered. Instead, the lyquid we expscted to frecture, with

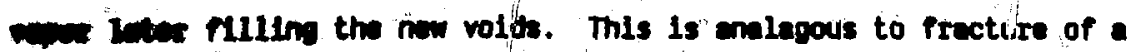


solid. The furpose of shis thesis is to expend these two viempoints, to use numerical experimints to justify a choice hotweripoprecties, and to recomend a method of calculating l:quid fregmentation in ICF reactors.

In Chapter Two, several ICF reactor dosions are described, end the degree of isochoric heating is est lmated for oach desion. In cheptor Three, the literature on liquid fregmentation in atomizer nozzles is reviewed and found to be unsuitable for prediction of respones to 1sochoric hoating. In Chapter Four, rupid vaporization is considured as a driving mechanism foc fragmentation. The pliase diagran for lithium is presented, and thermodynemic faths followed during and after the sudden heating are estlmated. Then, the alternative phase separation mechanisms of spinodal decomposition and of nucleation and growth are considered. Because the hydroctynemic expension of the liquid is faster than the vapor bubble growth time, nucleation and growth can not urive the fragmentatior. For lithium, the is ntropic spinodal line carl not be reached until the fluid expands by about $77 x$, and by that time, the (hydrodynamic) fragmentation process should be well advanced. Also, the energy used in phese change (assuming the vespor is In thermal equilibrium with the heated liquid) is much less tisan the expansion energy, and thus carnot be expected to be the driving mechanism for the expansion.

In Chapter Five, force balance and onergy minimization moveis (hydrodynomic models) of liquid fragmentation are doveloped and applied to the various ICF reacior desif .. Because these models do not consider the dynemic process of fragmentation, mumorical experiments were undertaken to study the fromomentation dynmics ess to 
test the predictions of the energy minimization model. These experiments used the tectnique of molecular dynanics to follow the evolution of up to several thousend liquid atoms following sudden hating. For simplicity, ard to better model the quasi-two-dimensional expension of en ICF reactor jet colum, the numrical experiments were performed in two dimensions using the Lennard-Jones (LJ) 6-12 interatonic potential. The phase diagran for this potential is shown in.Chepter Four; the two LJ constants are set to match lithium properties in the sume section. The energy minimization model is epplied to the LJ fluld in Chapter Five. The mumerical experiment tectniques are developed in Chapter Six and epplied in Chepter Seven. Chapter Seven also includes the significant result of this thesis: a comparison between the energy minimization model prodictions and the molecular dynemics experinents, with coments on the scalability of the model to reactor situations.

In Chepter Eight, the thesis is briefly sumarized and further mumerical simulations end laboratory experiments are recommended. Finilily, Appendix A is a draft journal article reporting on the results of the thesis. As such, it serves as an executive sumary, and it should probebly be read before proceeding to the remainder of the thesis. 
Chapter 2: Importance of Llquid Fregmentation to Inortinl Fusien Reactor Desion

All inertial confinement fusion (ICF) reactor desions are cyclic due to the pulsed nature of en Inertial fusion energy source. Bafore - fusion pulse cen be initiated by a laser or ion been, the chember pressure must be low cnough to permit beem propegation: $10 \mathrm{~Pa}$ $\left(-10^{-1}\right.$ torr $)$ for lasers and $10^{-2} \mathrm{~Pa}\left(-10^{-4}\right.$ torr $)$ for havy ion besws. In this chapter, the cyclic sequence of ovents is 1llustrated for on example reactor, several other reactors are described, and the degree of isochoric heating is calculated for each dasign.

The minimum chember pressure required for beem propegation is well above the vapor pressures of lithilum and $\mathrm{LI}_{17} \mathrm{Pt}_{83}$ (a 17 atonic percent lithlum, 83 atomic percent lead eutectic alloy), the two lithium bearing liquids that cen breed enough tritium to replace the tritlum burned in the plasma." At a working temperature of $500^{\circ} \mathrm{C}$,

* Tritium is burned in the plasma by the D-T reaction,

$$
{ }^{2} \mathrm{H}+{ }^{3} \mathrm{H} \rightarrow 4_{\mathrm{He}}+\mathrm{n}+17.6 \mathrm{MeV}
$$

Tritium is bred in the reactor lithium by two reactions,

$$
\begin{aligned}
& 6 \mathrm{LI}+\mathrm{n} \rightarrow{ }^{4} \mathrm{He}+{ }^{3} \mathrm{H}+4.8 \mathrm{maV} \\
& 7_{\mathrm{LI}}+\mathrm{n} \rightarrow{ }^{4} \mathrm{He}+{ }^{3} \mathrm{H}+\mathrm{n}-2.4 \mathrm{mVV}
\end{aligned}
$$

The $6_{\mathrm{Li}}$ reaction is most probable for thermalized neutrons, and $6_{\mathrm{LI}}$ is $7.4 x$ of natural 11 thium. The ${ }^{2} \mathrm{~L}$ reaction requires fast neutrons (E $2.4 \mathrm{MeV}$ ), and ${ }^{7} L 1$ is $92.6 \%$ of natural 1ithiun. 
lithium and $\mathrm{LI}_{17} \mathrm{~Pb}_{83}$ have vepor pressures of $0.4 \mathrm{~Pa}\left(3 \times 10^{-3}\right.$ torr) and $2 \times 10^{-3} \mathrm{~Pa}\left(1.5 \times 10^{-5}\right.$ torr $)$, respectively. It is highly adventapeous to utilize one of these liquids between the pulsed fusion plasm end the reactor structure since $\sim 1 / 3$ of the fusion enargy pulse is in the form of lons and $x$-rays wich are absorbed in a very thin layer. Absorption of these energy pulses directly in the structure produces eblation and unacceptable fatlgue loads unless the structure is very large ( $10 \mathrm{~m}$ radius). Conversely, absorption of the short-rande fusion energy pulses in liquid protects the structure. Furthermore, if the liquid layer thickness is $0.5 \mathrm{~m}$, sufficlent neutron shielding is provided to allow a $5 \mathrm{~m}$ radius structure to survive for the 30 year plant $11 \mathrm{fe}$, a distinct advantage over other fusion reactor designs which require replacement of reactor structures overy several years using remote maintenance equipment. Absorption of the ions and $x$-rays in liquid vaporizes and lonizes the thin absorbing layer which then causes vaporization of still more liquid by rapid thermal radiation heat transfer. The resulting gas density is much higher than the beem propagation limit, and it must be reduced by vacuum pumping or cooling and recondensation before the naxt driver pulse. If the bulk liquld fragnents, the Increased surface area reduces the cooling and condensation tines.

The HMIFE concept ${ }^{3}$ (High Yield Lithium cooled Inertial Fusion Enorigy converter) is used as en exemple throughout this report. HMIFE we chosen baceuse of Its extremely high deposited neutron enerey denily; if the HYIFE Iiquid doesn't fragment adequately, most ICF ranctor liquids also would not. The HMIFE gocmetry is shown in 
Fig. 2.1. The LNK HIIFE reactor concept. The lithium jet array absorbs most of the fusion energy, breeds tritium to replece tritium burned in the plasma, and shlelds the steel reactor structure from the fusion neutrons. The Jet array is completely repleced betwen fusion pulses.

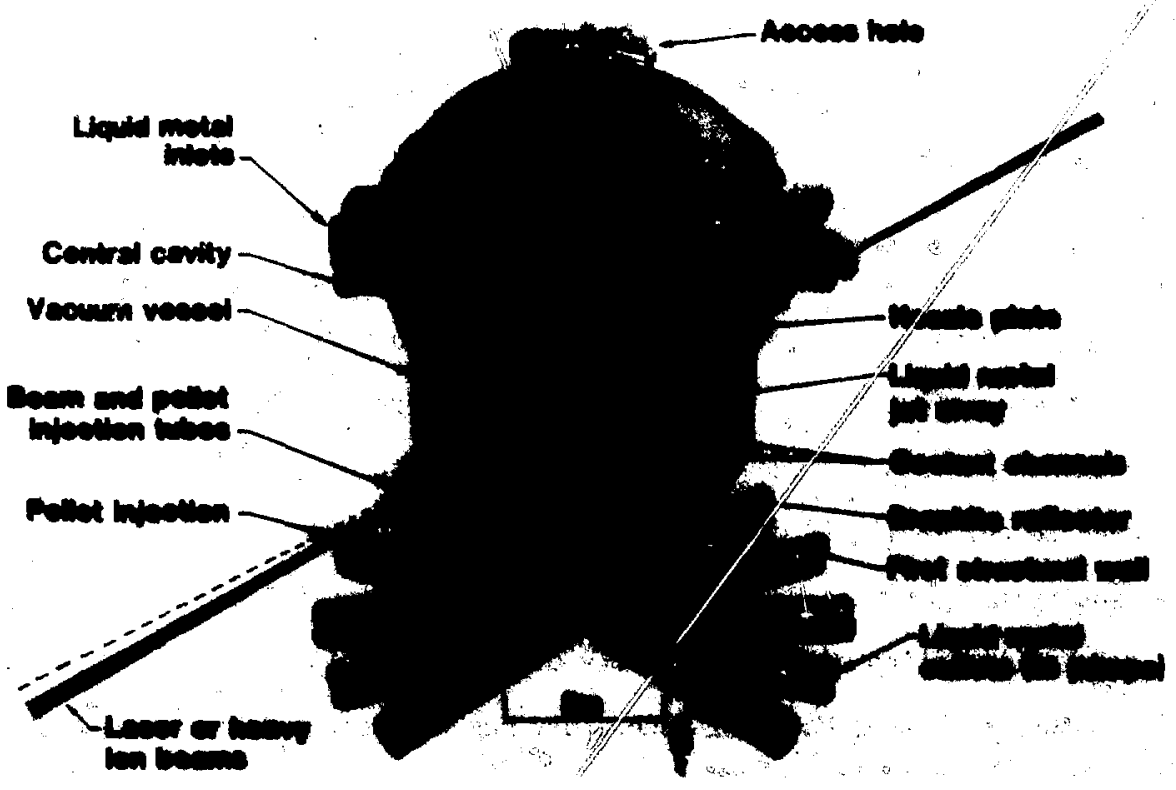


frealy through the $5 \mathrm{~m}$ radius chamer at radil between 0.5 and $2.0 \mathrm{~m}$. The fusion yield of $1800 \mathrm{~mJ}$ is pt:sed at $1.5 \mathrm{~Hz}$. The sequence of events in one pulse cycle of HYIFE is shown in Fig. 2.2.

The high yield in HILIFE coupled with the small liquid metal redius leads to extremly high energy density. The requirement to inject completely new jots every $2 / 3$ soconds implies a very high flow rate, $\sim 72 \mathrm{~m}^{3} / \mathrm{s}\left(1.1 \times 10^{6} \mathrm{gpm}\right)$. To reduce the flow rate, the University of wisconsin proposed in thoir HIBALL design' (FIg 2.3) that the liquid jets be loosely confined in woven SiC socks such that the liquid transit time through the chanber is increased to the optimum determined by the thermal cycle $(\sim 10 \mathrm{~s})$. Since the socks must contain the isochoric heating, the pulsed energy density in the liquid was reduced by increasing the liquid radius to between 5 and $7 \mathrm{~m}$ and by decreasing the yield to $400 \mathrm{~mJ}$. The reactor power was maintained by correspondingly increasing the pulse rate to $5 \mathrm{~Hz}$. (Subsequent Wisconsin calculations have limited the pulse rate to $3.3 \mathrm{~Hz}$ due to the cooling and condensation times ${ }^{10}$.) The eutectic $\mathrm{LI}_{17} \mathrm{~Pb}_{\mathrm{B} 3}$ liquid was used to reduce liquid matal fire hazards, but the increased fluid density (from 485 to $9320 \mathrm{~kg} / \mathrm{m}^{3}$ ) increased the pumping power and made satisfaction of seismic safety criteria for plping very difficult.

The Lus Alemos Mational Laboratory has proposed a Modifled Wetted wall desion (Mwir) ${ }^{11}$ (Fig. 2.4) in wich a 1 to 8-cm-thick lithium film flows on a $2 \mathrm{~m}$ radius wall. The reactor has $150 \mathrm{~mJ}$ fusion pulses at $10 \mathrm{~Hz}$. The Bachtel Group has proposed the EAGL reactor concept ${ }^{12}$ (Fid. 2.5) in wich light Ion beams propagate to the infected fusion

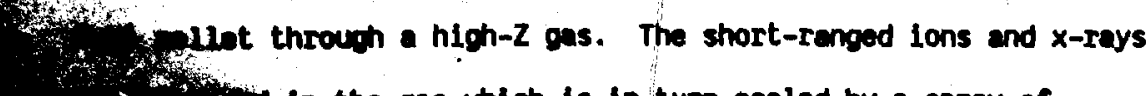
in the gas wilch is in turn cooled by a spray of 
Fig. 2.2. Times of interest for the $1.5 \mathrm{~Hz}$ mIfE reactor. The ges motion, liquid motion, and cooling times wre estimated from calculations in References 4 through 8.

-15 ms Deuterium-tritium fuel pellet enters the reaction chamber

-30 ns Pollet is 5 in from the chamber conter and the laser beems enter the chamber

$-15 \mathrm{~ns}$ Laser beem contacts the fuel pellet

- Implosion complete, fusion begins

10 ps Fusion burn complete

2 ns $X$-rays begin to ablate lithium

1 us Neutron absorption complete

20 us $X$-ray and thermally ablated lithiun reaches maximum compression

$40 \mu \mathrm{s}$ Jet fragmentation due to neutron absorption begins

60 us Re-expanding lithium gas reaches the fragmenting jets

300 us Expanding lithium gas clears the fragmenting jets

400 us Lithium gas impacts wall

$35 \mathrm{~ms}$ Lithlum liquid impacts wall

$250 \mathrm{~ms}$ Chamber pressure reduced to $10 \mathrm{~Pa}\left(10^{-1}\right.$ torr $)$

630 ms New lithium jets established

652 ms Next fusion fuel pellet enters the chamber

$666+$ ms Next laser bean enters the chanber

$666+$ ins Next fusion begins 
F1g. 2.3." The Wisconsin HIBALL reactor concept. The woven SiC tubes contain $\mathrm{LI}_{17} \mathrm{~Pb}_{83}$ that ebsorbs the fusion energy, breeds the tritium, and shields the reactor structure. The SIC reduces the Ilquid flow rate to the value required by the thermal cycle.

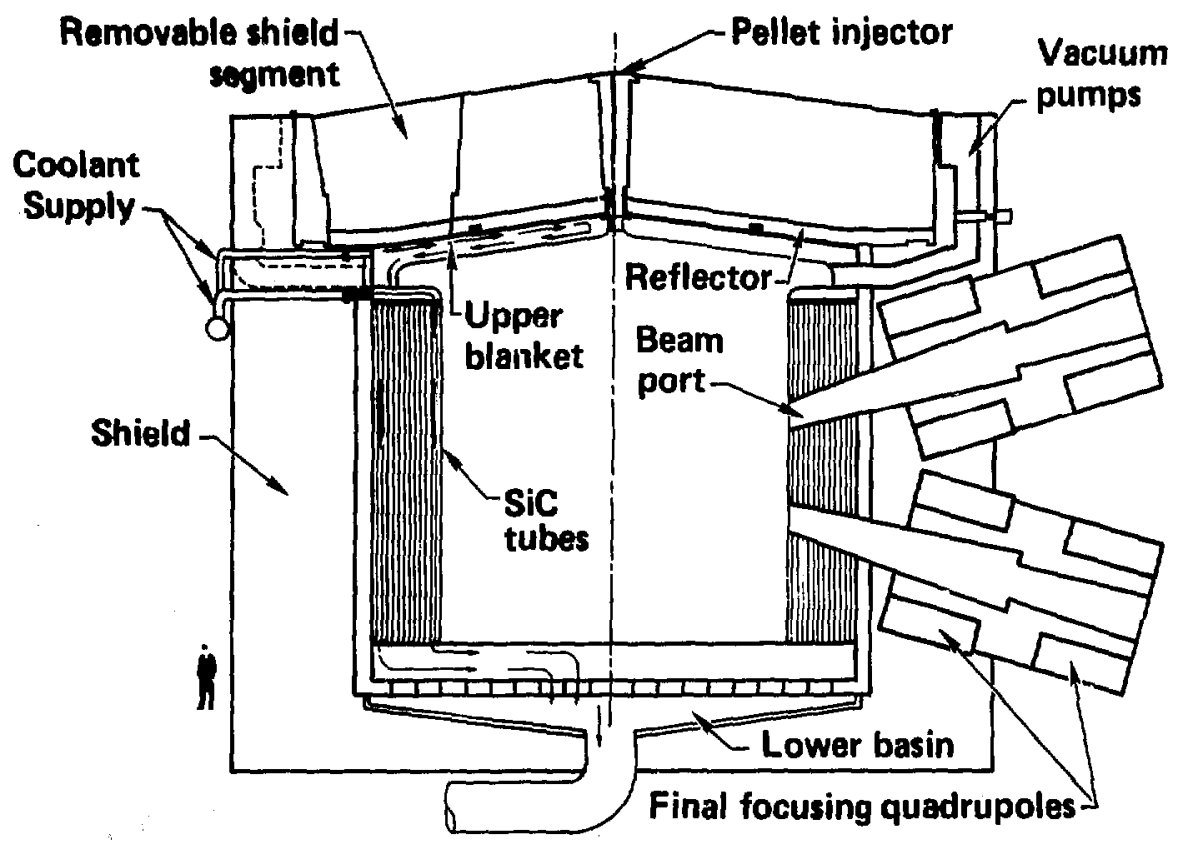


Fig. 2.4. The Lak Modified wetted Wall reactor concept. The liquid lithium film absorbs the short-ranged lons and $x$-rays, but it does not protect the inner structural wall from neutron damage. The majority of the neutron energy is absorbed behind the wall in the lithlum blanket.

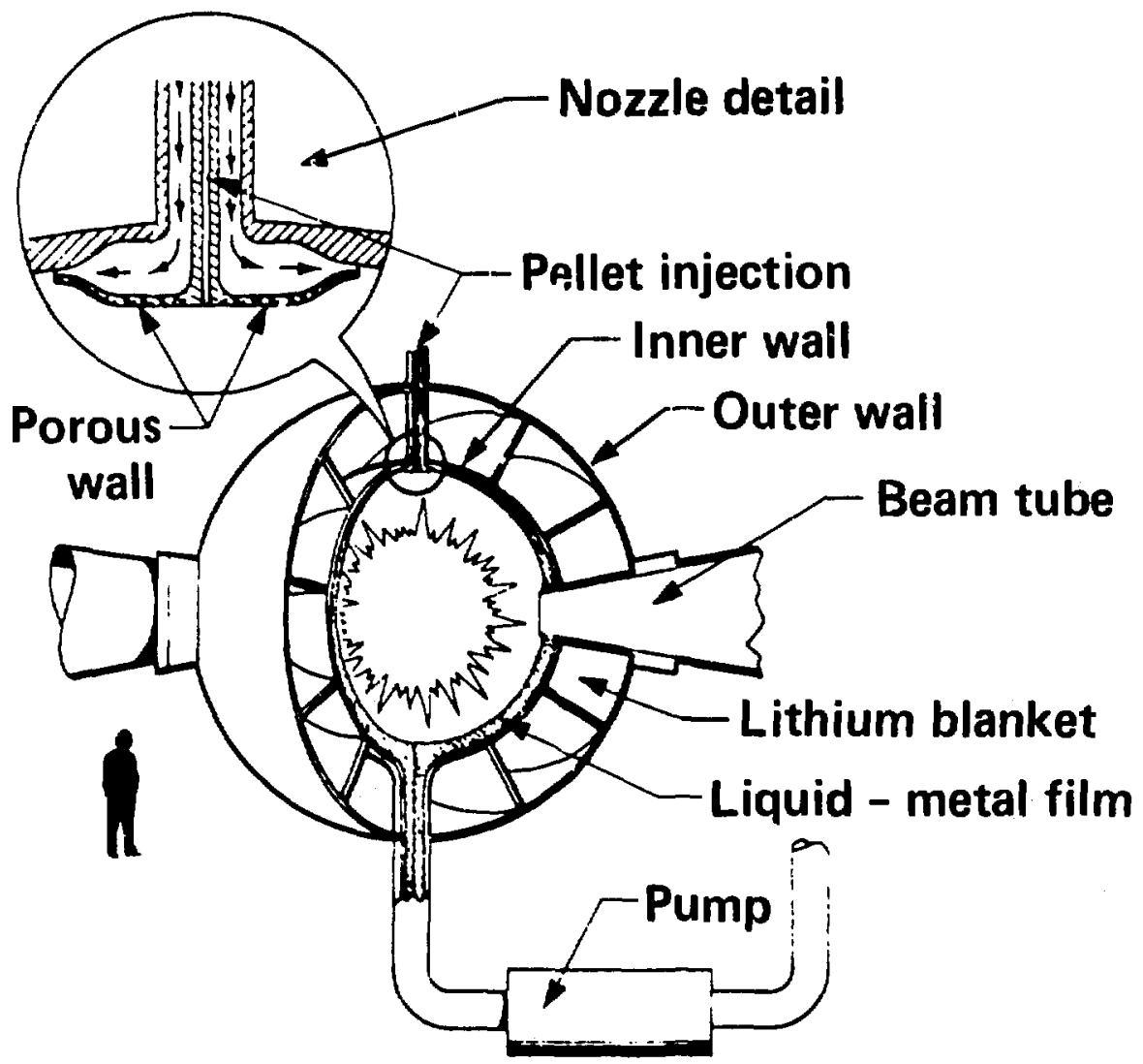


Fig. 2.5. The Bechtel EAGLE reactor concept. The chamber gas absorbs the short-ranged lons and x-rays, and the lithium spray cools the gas. The majority of the neutron energy is absorbed behind the wall in the blanket nodules.

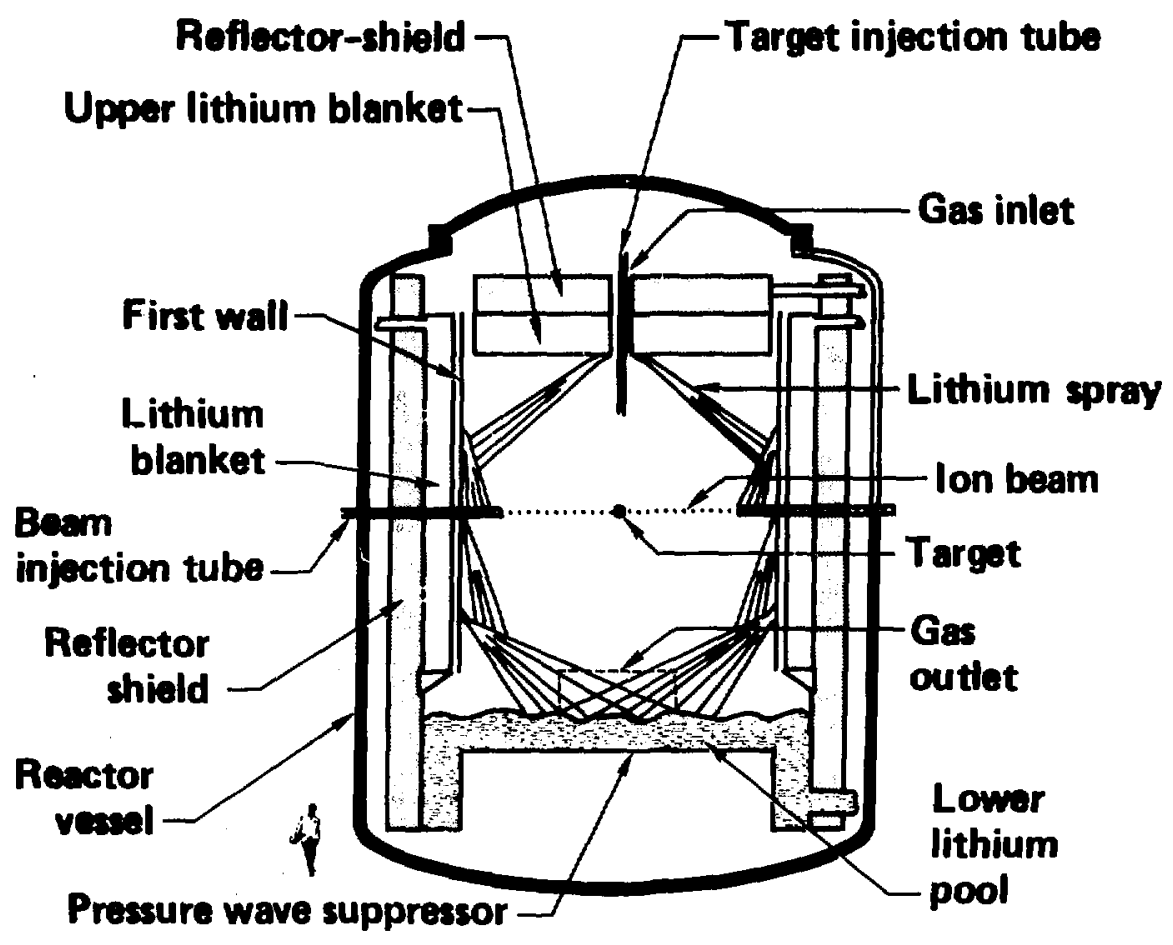


0.5-min-diemeter lithium droplets at radil of $3 \mathrm{~m}$ to the $4-m$-radius wall. The fusion yield of $300 \mathrm{MJ}$ is pulsed at $3 \mathrm{~Hz}$. LLW has proposed the Pulse"Star ${ }^{13}$ reactor concept (Fig. 2.6) in wich a spray of $1 \mathrm{~L}_{17} \mathrm{~Pb}_{83}$ absorbs the fusion neutrons. The spray is confined between a $4 \mathrm{~m}$ radius screen and a $5.5 \mathrm{~m}$ radius belljar. The wetted screen absorbs the lons and $x$-rays and the spray absorbs the neutrons. The belljar separates the spray-vacuum region from the outer $\mathrm{LI}_{17} \mathrm{~Pb}_{83}$ pool. Use of a pool eliminates solsmic desion difficulty associated with high density liquid plpe loops. The fusion yield is $600 \mathrm{MJ}$ at $5 \mathrm{~Hz}$.

In some of these reactor concepts, the liquid metal residence time is less than the interpulse time; in others, it is many times longer. The liquid metal geometries include free spheres, free cylindrical jets, cylindrical jets confined by porous tubes (with some liquid film on the exterior surface), and liquid film on the inside surface of a sphere. Two different liquid metals have been suggested, and the fusion ylelds vary greatly. Thus, any analytic or empirical theory of 1sochoric heating-induced fragmentation must span a large parameter space.

Neutron transport computer codes have been used to calculate the time and space dependent neutron energy deposition levels in several of these reactors. Flgure 2.7 shows the temporal neutron energy deposition profile for the HYI.IFE reactor. The deposition time in any particular region is -0.1 to 1 us since the profile was spatlally integrated over the entire blanket. Similar results are expected for the other reactor geometries. The time for relief of the Induced pressure is the liquid thickness (or diameter) divided by the sound 
Fig. 2.6. The UMK Pulse*Star reactor concept. The wetted screen absorbs the $x$-rays and lons. The spray absorbs the neutrons from 50 pulses during its transit through the reactor.

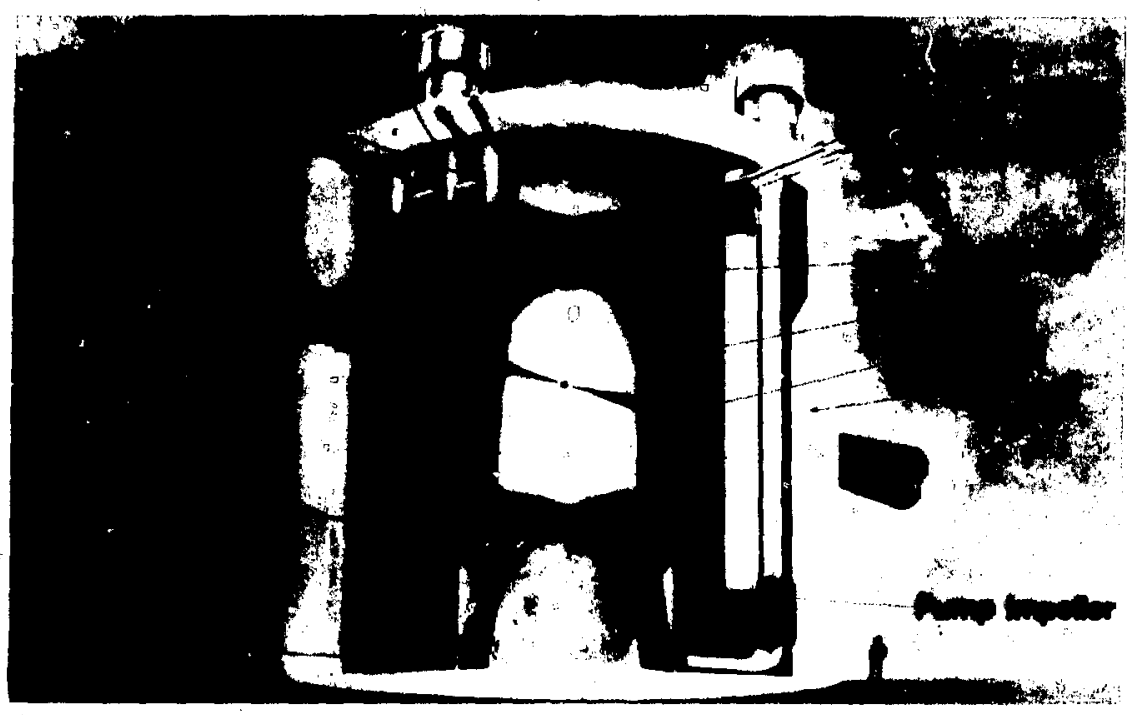


F1g. 2.7. Combined neutron and game energy deposition in the HMIFE lithium jet array as a function of time.

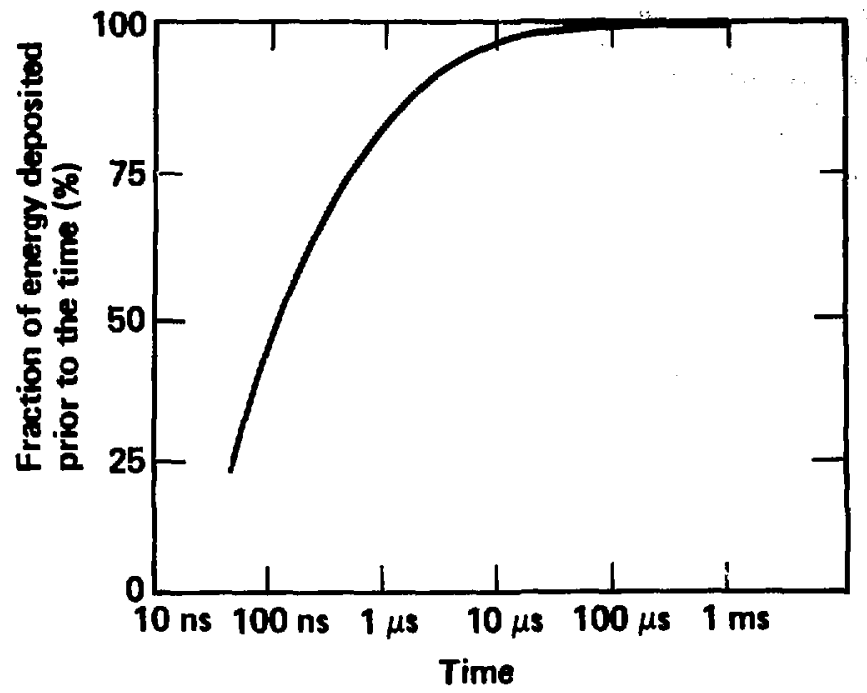

Fig. 2.8. Neutron and gamna energy deposition profile in the HYI.IFE lithium jet array.

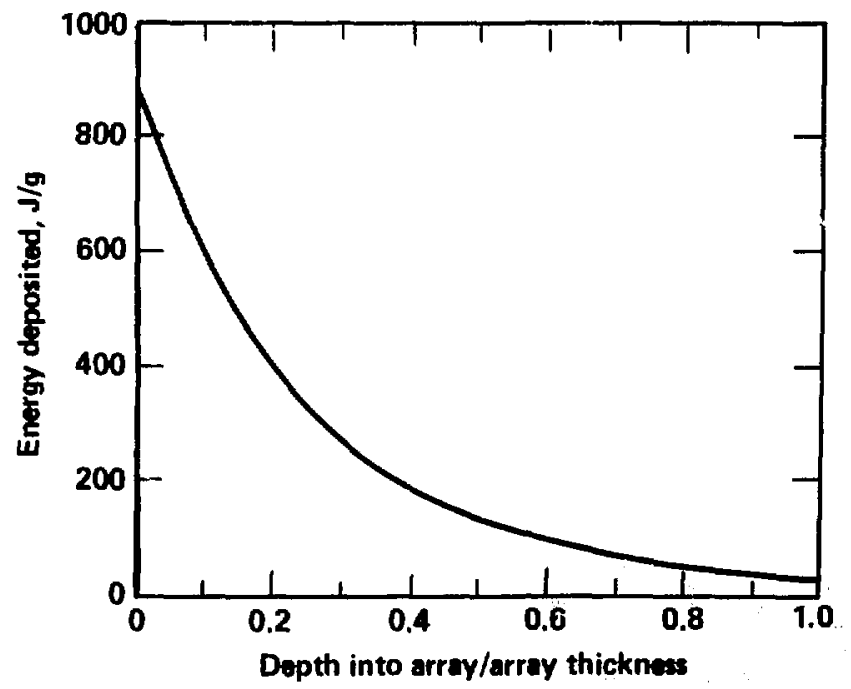


spead. As shown in Table 2.1, the sound speeds, c, of lithlum and $\mathrm{LI}_{17} \mathrm{Ph}_{83}$ are 4.5 and $1.8 \mathrm{~km} / \mathrm{s}$, respectively. The healing is "isoctioric" if

$$
\frac{2 \mathrm{R}}{C}=2 \tau>\tau_{\text {dep }}=0.1 \mathrm{usec} .
$$

That 1s, for isochorlc hoating, the minimun radius of the liquid is

$$
R_{m L n}=\left\{\begin{array}{l}
0.25 \mathrm{~mm} \text { for } \mathrm{LI} \\
0.1 \mathrm{mim} \text { for } \mathrm{LL}_{17} \mathrm{~Pb}_{83}
\end{array}\right.
$$

Table 2.1. Fluid properties at $500^{\circ} \mathrm{C}$.

Property

Symbol

Sound speed

Mass density

Gröneisen parameter

Surface tension

Vapos pressure c

c $\mathrm{m} / \mathrm{s}$

$P_{m}$

$\Gamma$

$Y$

$P_{\mathbf{g}}$
Unlts

$\mathrm{m} / \mathrm{s}$

$\mathrm{kg} / \mathrm{m}^{3}$

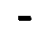

$\mathrm{J} / \mathrm{m}^{2}$

$\mathrm{Pa}$

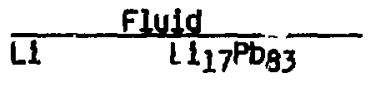

Fluid

$\mathrm{II}_{17} \mathrm{~Pb}_{83}$

1800

9.320

2.0

0.435

0.002

Floure 2.8 shows the spatial neutron energy deposition profile in the HMIFE reactor. The neutron energy deposition drops to $35 \%$ of the peck value ecross the inner 20-cm-diemeter jets, and is a factor of $\sim 15$ lowr in the outer jets. The leading edge value of $\sim 800$ kJ/kg cen be usod to calculate the pressure rise from the Groneisen equation of state, 
where $\rho_{m}$ is the mass density (485 and $9320 \mathrm{~kg} / \mathrm{m}^{3}$ for lithlim and $1.1_{17} \mathrm{~Pb}_{83}, \Gamma$ is the Grinelsen parameter $(1.0$ and 2.0 enr 11 thilin and I. $\left.1_{17} \mathrm{~Pb}_{83}\right)$, and $e$ is the speciflc energy deposited in J/kg. For HM IFE, the peak neutron induced pressure is then $400 \mathrm{MPa}$ or $4 \mathrm{kbar}$. The neutron energy deposition per unit volume scales roughly with $\left(\phi_{V} Y / R_{L}^{2}\right)$ where $Y$ is fusion yield, $R_{h}$ is the blankei radius, wid $p_{V}$ is the volume fraction of absorbing material. To scaie from lithium ti $\mathrm{LI}_{17} \mathrm{~Pb}_{83}$, the volumatric deposition is increased by another factor of $\sim 6$. This scaling and the published meutron trensport results for the other reactor geometries were usad tosalculate the induced pressures and rellef times for each geometry (Table 2.2). The jump-off velocity, $v$, of the expanding liquid can be calculated by considering the pressure drop across a moving rarefaction wave. Ahitad of the wave, the fluid is at rest. In the frame of reference of the wave, it has a velocity of $(-c)$. Behind the wave, the fluid has a velocity of $(v-c)$ in this frame. The momentum conservation equation is

$$
\begin{aligned}
& \Delta P+\left(-p_{m} c\right)(v-c)=0+\left(-p_{m} c\right)(-c) \\
& \Delta P=P_{m} c V,
\end{aligned}
$$

since the mass fl.ux is $\left(-\rho_{m} c\right)$. For HYLIFE, the $400 \mathrm{MPa}$ pressure results in a $180 \mathrm{~m} / \mathrm{s}$ Jump-off velocity. Table 2.2 also shows the Jump-off velocitles for the other reactor concepts. 
Table 2.2. Isochoric heating in several ICF reactors.

\begin{tabular}{|c|c|c|c|c|c|c|}
\hline & $\underset{\text { Jot }}{\text { Inner }}$ & Outer & $\begin{array}{c}\text { HIBALL } \\
\text { Inner } \\
\text { Jatt }\end{array}$ & $\begin{array}{l}\text { Modified } \\
\text { Wetted } \\
\text { Wall }\end{array}$ & EACLE & Pulse*Star \\
\hline $\begin{array}{l}\text { Yield } \\
\text { (MJ) }\end{array}$ & 1800 & 1800 & 400 & 150 & 300 & 600 \\
\hline $\begin{array}{l}\text { Pulse } \\
\text { Rate } \\
\text { (Hz) }\end{array}$ & 1.5 & 2.5 & 5 & 10 & 3 & 5 \\
\hline Fluid & LI & LI & $1.1_{17} \mathrm{~Pb}_{83}$ & $3 \mathrm{LI}$ & Li. & $\mathrm{Li}_{17} \mathrm{~Pb}_{83}$ \\
\hline $\begin{array}{l}\text { Thickness } \\
\text { (cm) }\end{array}$ & 20 & 20 & 3 & $1-8$ & 0.05 & 1 \\
\hline $\begin{array}{l}\text { Relief } \\
\text { Time } \\
\text { (us) }\end{array}$ & 44 & 44 & 17 & $2-18$ & 0.1 & 6 \\
\hline $\begin{array}{l}\text { Specific } \\
\text { Energy } \\
(J / g)\end{array}$ & 800 & 50 & 0.5 & 3.5 & 3.1 & 1 \\
\hline $\begin{array}{l}\text { Pressure } \\
\text { (MPE) }\end{array}$ & 400 & 25 & 9 & 1.8 & 1.9 & 19 \\
\hline $\begin{array}{l}\text { Jupp-off } \\
\text { Yelocity } \\
(m / s)\end{array}$ & 180 & 11 & 0.5 & 0.8 & 0.9 & 1.1 \\
\hline
\end{tabular}

+ Ionoring SIC sock 
Chapter 3: Liquid Fragmentation in Atomizer Nozzles

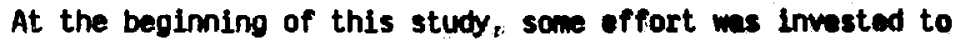
determine if violent fragmentation of liquids has tean studied in other applications. One significant body of literature studies the breakup of liquid jets into fine droplets as in diesel. engine fuel injectors. In this chapter, the literature is brlofly sumarlzed and evaluated to determine Its applicabslity to breekup of liquids in Inertial confinement fusion reactors.

In diesel engine fuel injectors, breakup of the fuel jet into snall fragments is desirable to promote ignition and uniform combustion. There is extensive literature on this subject; for example, references 14 through 16 and the references cited therein. Typical jets are injected with a driving pressure of $\sim 20 \mathrm{MPa}$ into a chamber pressure of $\sim 3 \mathrm{MPa}$. As the jet velocity is increased, four distinct breakup modes are observed ${ }^{15}$. At low velocity, surface tension breaks the jot into a series of spheres with dieneters somewhat larger than the jet diameter (the Rayleigh breakup regime). At higher velocity, the inertial effect of the surrounding gas accelerates this breakup into drops, still with diameters of the same order as the jet diameter (first wind-induced breakup regime). At still higher velocities, the surrounding gas creates short-wavelength three-dinensional waves on the jet surface mich causes breakup downstreem of the nozzle into a spray of fine droplets (second wind-induced breakup regine.) Finally, at very high velocities, the jet breaks up at the nozzle exit into a diverging spray of very small droplets (the atomization regime). Typical atomization droplet sizes 
from mon size nozzles are tens of microns ${ }^{14}$.

The various theories of atomization ${ }^{16}$ invoke several possible mechenisms including aerodynamic interactions, llquid turbulence, velocity profile rearrangement, supply pressure oscilllations and cavitation. Experiments ${ }^{15,16}$ have shown that only cavitation within the nozzle is capable of producing atomization in the absence of the other phonomona. However, the possibility that a combination of two or more of the other mechanisms could cause atomlzation was not ellminated by the experiments.

It does not eppear that the atonization literature can be used to prodict isochoric heating-induced breakup. The theory is uncertaln, and the literature is largely empirical. In addition, the boundary conditions are different, and the correspondence of inlet nozzle conditlons to isachoric heating heating conditions is not easily determined. 
Chepter 4: Repid Veporization as a Fragnentation mochenism

At the begining of this study, two potential ceusas of liquid fregmentation in inertial fusion reactors wre proposed." One, initially proposed by Lewis Glem of LLK, sugaested that the isochorically heated liquid would expend homogeneously due to the tensile relief wave propagating inward from the free surface. The tension would fracture the liquid into fragments and vold. The volds would gradually fill with vapor, either from the vapor originally surrounding the liquid in the reactor or from the free surfaces of the newly created fragnents.

The second mechanism, initlally proposed by Vijay Kulkarny of TRW, suggested that the homogeneously expanding liquid would quickly reach a state under the liquid-vapor two phase dome where rapid phase change could occur, probably by the process of spinodal deconposition. Then, the growing vapor reglons would accelerate the remaining liquid which would be in the fragments.

This chapter explores the latter (rapid phase change) mechanism. I.Ithlum in the HYLIFE reactor is used as an example. The known lithium equation of state is used to construct a phase diagran. The thermodynemic paths followed during isochoric heating and subsequent homogeneous expansion into the netastable region under the two phase .Liquid-vapor done are determined. The two possible phase chenge mechanisms to restore equilibrium, spinodal decomposition and nucleation and growth, are reviewed. Finally, rapid phase change is evaluated as a cause of fragmentation. The conclusion is that both phase change mechanisms will be too slow to cause the fregmentation. 
In view of this conclusion, hydrodynamic modeling and molecular dynaics simulations were initlated; these are described in Chapters Five, Six, and Seven. The simulations used a simplified fluld (6-12 Lennard-Joneslum) in two dimonsions. The known phase diagrem for this fluld is also summrized in this chapter, and analytic equations are fit to the Lennard Jones equation of state data. 


\subsection{Lithilu Phase Diagram}

In 1977, Young reported an equation-of-state for lithium. 17 The equation of state was used in Glemn's AFTON code ${ }^{18}$ calculations ${ }^{4-8}$ of the HYLIFE inertial fusion reactor hydrodynamics. This section summarizes Young's davelopment of the equation of state. The soft sphere model of Hoover, et. al. ${ }^{19}$ is used. This model is based on Monte Carlo calculations for particles interacting with repulsive pair potentials of the form

$$
\phi(r)=+\left(\lambda_{I} / r\right)^{\xi}, 4 \quad 5 \quad 12
$$

where $\lambda_{I}$ is the interatomic spacing at which the potential is $t$.

The Helmholtz free energy for Young's equation of state is

$$
\begin{aligned}
A & =N_{n} k T\left[-\ln \left(\frac{V_{e}^{*}}{N \lambda_{d}^{3}}\right)+C_{n} \rho_{y}^{\xi / 3}\left(\frac{\hbar}{k T}\right)+\frac{(E+4)_{Q_{e}} \rho_{y}^{\xi / 9}\left(\frac{k}{k T}\right)^{1 / 3}}{2}\right. \\
& \left.=p_{y}^{n}\left(\frac{t}{k T}\right)\right]+E_{c o h} .
\end{aligned}
$$

In the brackets, the first term represents the ideal gas free energy, the second term is the static lattice potential energy, the third term is the thermal lattice potential energy correction, and the fourth term is the attractive potential energy. In the first term, $\lambda_{d}$ is the de Broglie wavelength, $h /(2 . m k T)^{1 / 2}$. In the second term, $C_{n}$ is the face centered cubic Madelung constant", and $p_{y}$ is the normalized density, $\left(N_{m} / V\right)\left(R_{r}^{3} / \sqrt{2}\right)$. In the third term, $Q_{e}$ is a multiplier to account for electronic effects on the heat capacity. Since the form of the attractive part of the effective potential in metals is complex and poorly known, a man-fleld term is used as an approximation in the fourth term. The final term outside the 
brackets, $E_{\text {coh' }}$ is the cohesive energy, 1.e., the energy required to convert zero Kelvin lithium to completely separated atoms.

The pressure, energy, and entropy may be found from

$$
\begin{aligned}
& P=-\left(\frac{\partial A}{\partial V}\right)_{N, T} \\
& E=A-T\left(\frac{\partial A}{\partial T}\right)_{N, T} \\
& S=-\left(\frac{\partial A}{\partial T}\right)_{N, T} .
\end{aligned}
$$

The results of the differentiations are

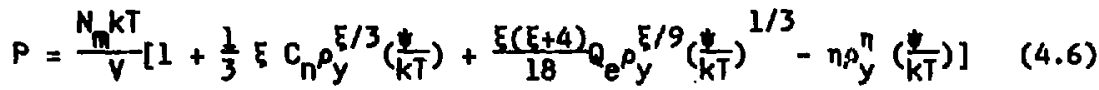

$$
\begin{aligned}
& E=N_{m} k T\left[\frac{3}{2}+C_{n} \rho_{y}^{\xi / 3}\left(\frac{t}{k T}\right)+\frac{(\xi+4)}{6} Q_{e} \rho_{y}^{\xi / 9}\left(\frac{1}{k T}\right)^{1 / 3}-\rho_{y}^{n}\left(\frac{1}{k T}\right)\right]+E_{c a h}(4.7) \\
& S=(E-A) / T
\end{aligned}
$$

Eqs. 4.6 and 4.7 are equivalent to Young's Eqs. 4 and 5.

The parameters $\hbar, \lambda_{r}, \xi, \eta$, and $\eta_{e}$ are adjustable and are chosen by fitting avallable enthalpy, volume, and sound speed 1sobars. Also, $\xi$ is chosen such that $(\xi+2) / 6 \approx r_{0}$, the Groneisen parameter at normal solid volume, as predicted by quasi-harmonic lattice theory. Simllarly, $n$ should be $\sim 1.0$ to agree with van der Waals equation of state and its good fits to molecular fluid data. Finally, $a_{e} \sim 1$ except were electronic corrections are clearly warrented.

Young's best fit values for $L I$ are

$$
E=4, n=0.7, D_{a}=1.30 \text {, }
$$




$$
\begin{aligned}
& t=1.99436 \times 10^{-17} \frac{\mathrm{J}}{\mathrm{atom}} \text { and } \\
& \lambda_{\mathrm{r}}^{3} / \sqrt{2}=1.29408 \times 10^{-31} \mathrm{~m}^{3} / \mathrm{atom} .
\end{aligned}
$$

Comparisons of the equation of state and experimental date are shown in Fig. 4.1 for enthalpy, thermal expension, and sound speed; the agreament is reasonable. The equation of state also produces critical point valuas of $T_{c}=3741 \mathrm{~K}, P_{c}=114 \mathrm{MPa}, V_{c}=5.86 V_{0}$ ' and $Z_{c}=P_{c} V_{c} / N_{p} k T_{c}=0.28$. The predicted Grinaisen constent at $V_{0}$ is 1.0 (comparable to a neasurenent of 0.9 ).

The Independent varlables are $V\left(m^{3} / \mathrm{kg}\right)$ and $T(K)$. Useful $L 1$ data are sumarized in Table 4.1. After inserting the constants, Eqs. 4.2, 4.3, and 4.4 becone

$$
\begin{aligned}
& A\left(\frac{J}{k g}\right)=1198.16 \mathrm{~T}\left[\ln \left(107.573 \mathrm{VT}^{3 / 2}\right)+4.60365 \mathrm{~V}^{-4 / 3} \mathrm{~T}^{-1}\right. \\
& \left.+3.71071 V^{-4 / 9} T^{-1 / 3}-495.542 V^{0.7} T^{-1}\right]+2.303 \times 10^{7} \\
& P\left(\Gamma_{A}\right)=1198.16 \mathrm{TV}^{-1}\left[1+6.13820 \mathrm{~V}^{-4 / 3} \mathrm{~T}^{-1}+1.23690 \mathrm{~V}^{-4 / 9} \mathrm{~T}^{-1 / 3}\right. \\
& \left.-346.8 / 9 y^{-0.7},-1\right] \\
& E\left(\frac{J}{k g}\right)=1198.16 T\left[1.5+4.60365 v^{-.4 / 3} T^{-1}+1.23690 V^{-4 / 9} T^{-1 / 3}\right. \\
& \left.-495.542 v^{-0.7} T^{-1}\right]+2.303 \times 10^{7} .
\end{aligned}
$$

When Eq. 4.10 is plotted as a set of 1sotherns in the P-V plane, a graph s.imilar to Fig. 4.2 results. In the two phase region, the isotherms form Van der Waals loops. These are considered to be analytic continuations of the isotherms into the two phase region; equilibrium isotherms are horizontal in the two phase region. For the critical isotherm, the loop vanishes at the point were both the first 
Fig. 4.1. Soft-sphere fit (smooth curves) to experimental. Iithium thermodynamic data (points). (From Ref. 17.)

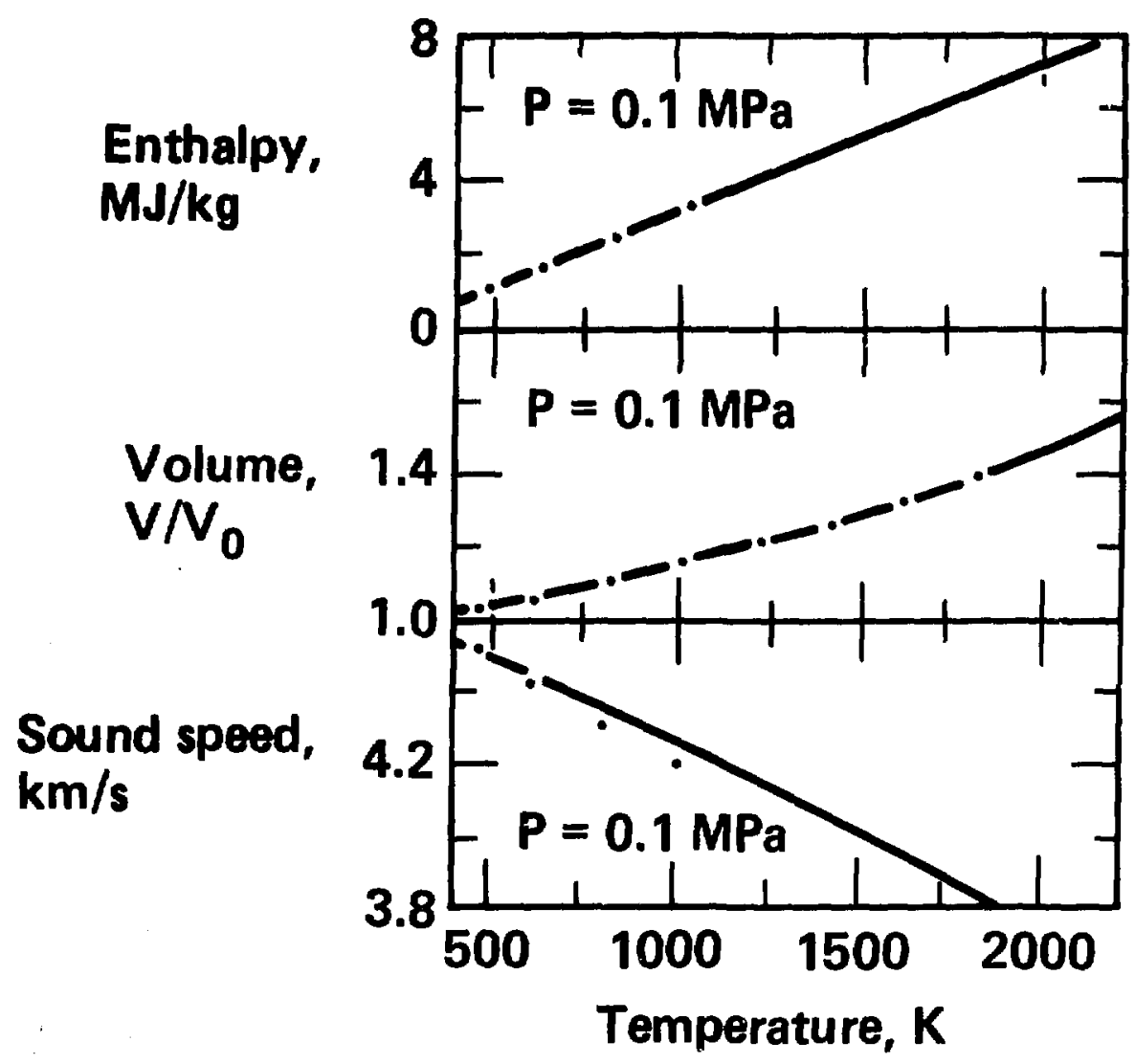


Table 4.1. I.1thium data.

LI atomic weight $=0.006939 \mathrm{~kg} / \mathrm{mol}$

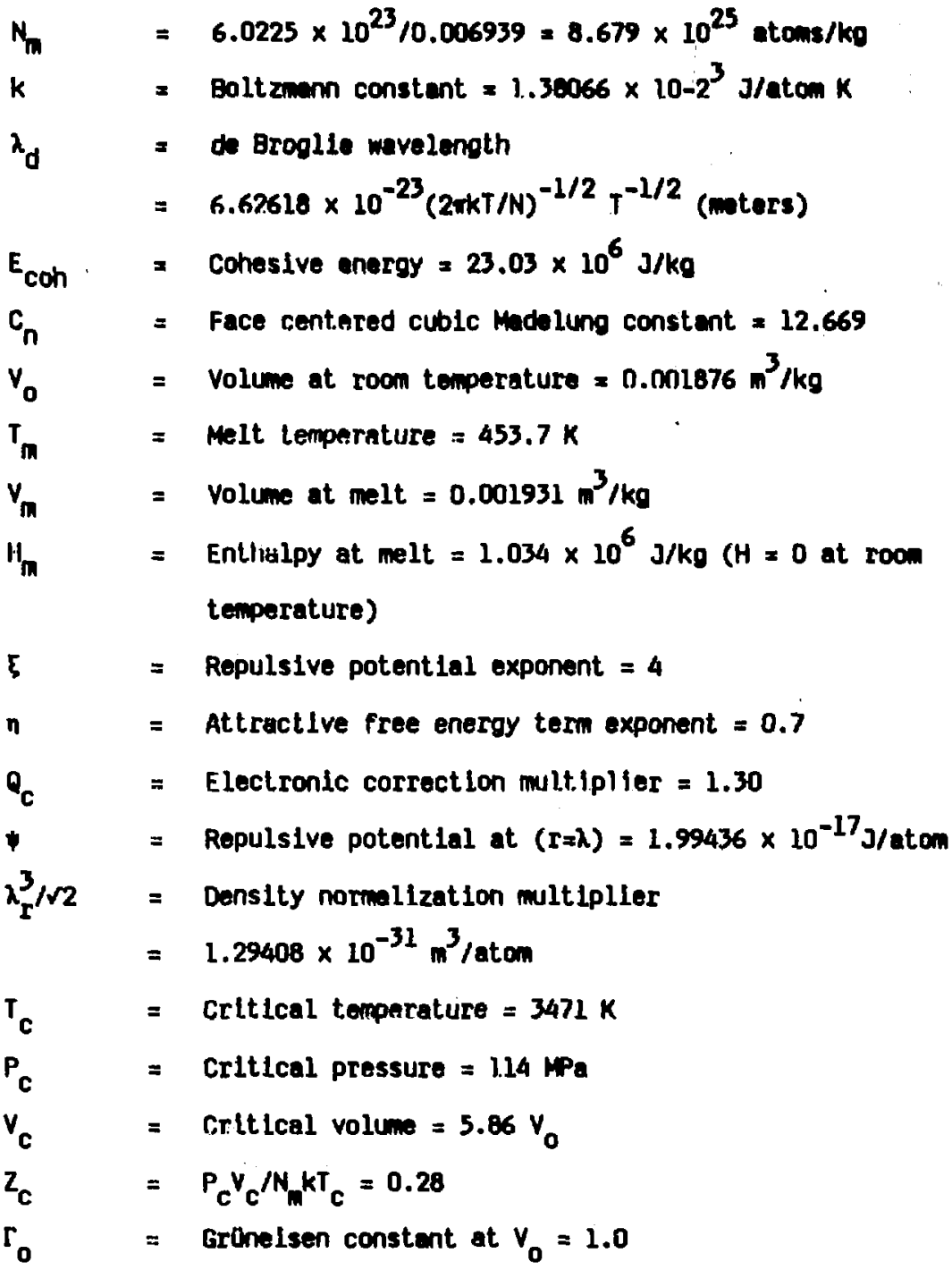


Fig. 4.2. Equilibrium and nonequilibrlum lsotherms on a schematic P-V diegram. Shaded regions approach equilibrium by nucleation and growth. The central unshaded region separates to the isothermal splnodal points by spinodal decomposition. The specific volume scale is highly nonlinear; see Fig. 4.4 for a portion of the l.1thium P-V diagran with a linear abscissa.

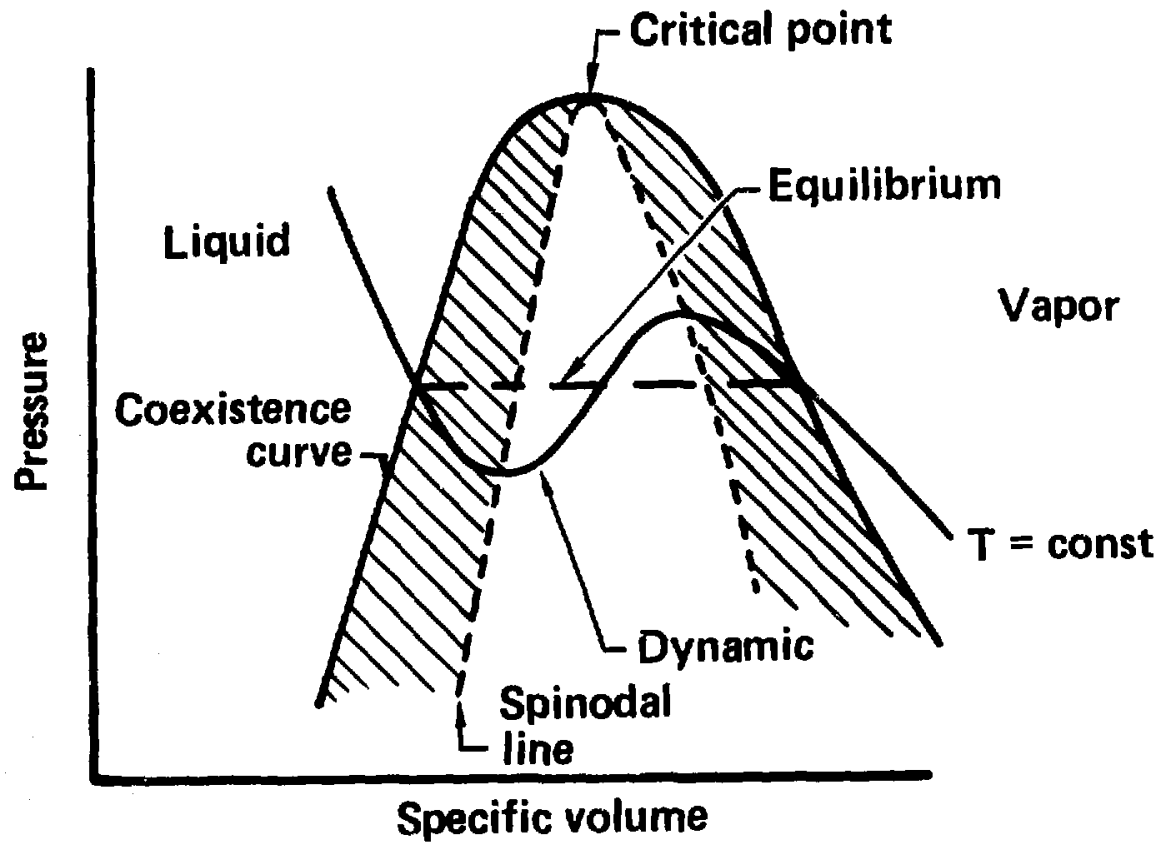

Shaded regions approach equilibrium by nucleation and growth.
Central unshaded region separates to the spinodal points by spinodal decomposition 
and second derivatives, $(2 P / \partial y)_{T}$ and $\left(\partial^{2} P / \partial v^{2}\right)_{T}$, are zero.

Isotherms above the critical temporature have $(2 P / O V)_{T} 0$ everyntere. If a liquid is converted to a gas along a path lying above the critical point, no phase separation is observabis; instead, the fluld changes gradually from a liquid to a gas without. avolution of bubbles. On each lsotherm below the critical temperature, thare are two specific volumes at wich (aP/OV) ${ }_{T}$ vanlshes; these polnts are known as the 1sothermel spinodes. There are analogous isentroplc spinodes where (JP/JV) $\mathrm{S}$ vanishes. The locus of all the spinodes defines the spinodal. IInes. For states between the two splnodal lines, a homogeneous fluid would be mochanically unstable, with (aP/OV) $)_{T} 0$. Hence, If a matiertal is suddenly transformed to a state between the spinodal lines, it is unstable, and it will decompose in a very short period (prohably subnanosecond) to a mixture of the two adjacent spinodes. (See Section 4.4 for a more detalled discussion of spinodal decomposition.) Phase separation would then continue by the slower process of growth unt.ll the two coexisting equitibrium states of liquid and vapor are raached. Similarly, if a material is suddenly transformed to a metastable state between one spinodal line and its assoclated coexistence rurve, phase separation would eventually occur by the sequential processes of mucleation and growth (see Section 4.5).

The saturated liquid and vapor lines (also knom as the coaxistence rurve or the liquid-vapor two-phase-dome) are constructed by computing the two phase area under each isotherm for a range of pressures. For each computation, the pressure axis is renormalfzed such that the 
Fig. 4.3. Iilustration of Maxwell's equal area rule for determining the coexistence curve. For each temperature, the positive and negative areas are equal for only one pressure, the equilibrtum pressuro.

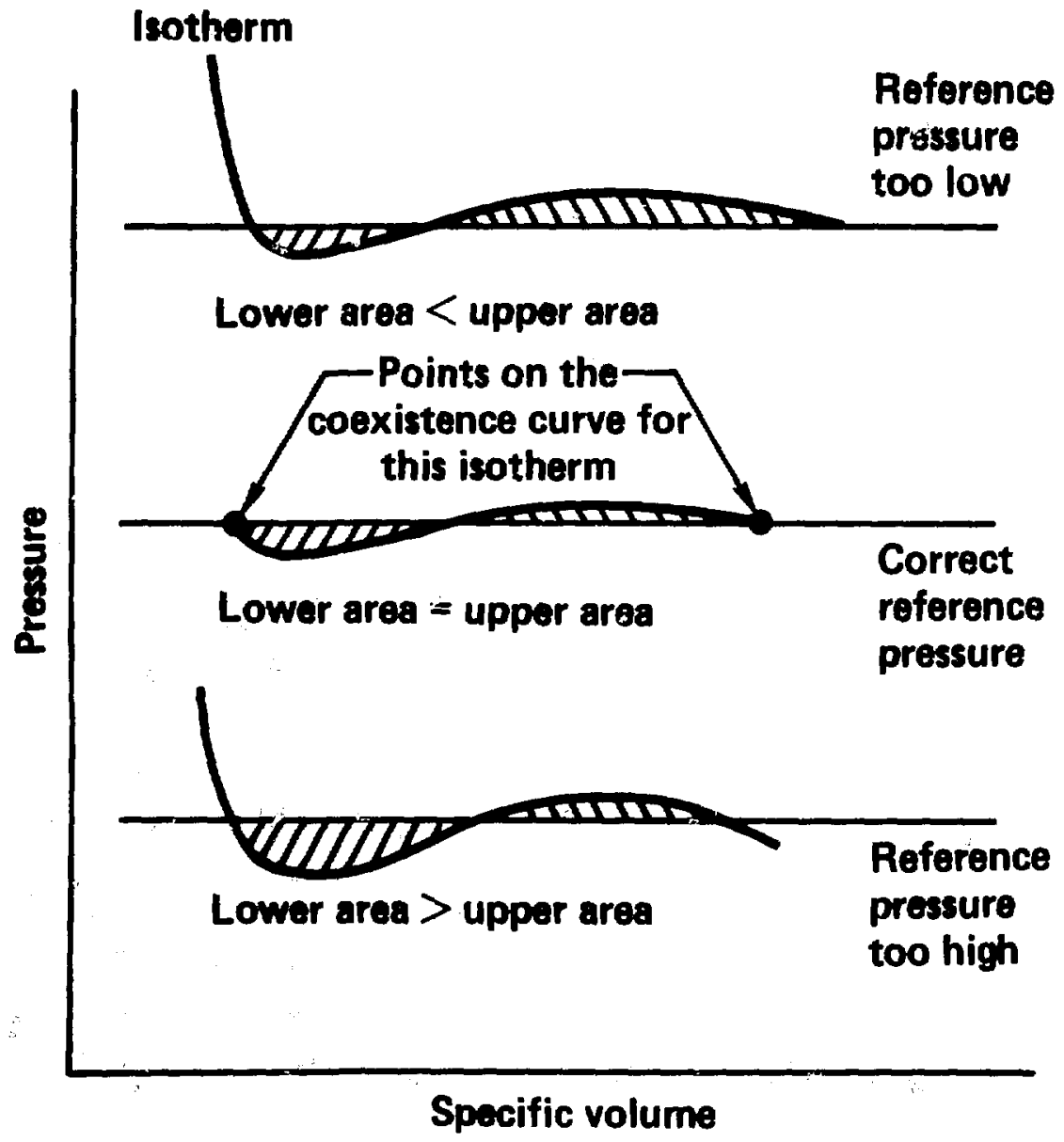


selected pressure is labeled as żero. "For eech lsothem, there is only one pressure where the positive and negitive contributions to the area total to zero; this is the equllibrium pressure (Fig: 4.3). The twn intersections of the 1sotherm and this oquilibrium pressure lis on the salurated liquid and vepor lines. This property of the coexistence curve is known as Maxwell's equal area rule. For the two phases to be In thermal and mechanical oquilibrium, they must be at the sene temperatilire and pressure (to prevent mergy and momentum excharige, respectlvely). They must also be in chemlcal equilibriun to prevent mass exchange.

$$
I d G=0=I V d P-I S d T+\sum \mu_{1} d n_{1}
$$

For phase equilibrlum, each $n_{1}$ is constant, and the last term is zero. Constant temperature is implfed by the lack of energy flow. Thiss,

$$
\int d \mathbf{G}=\mathbf{D}=\mathbf{S} \mathbf{d p} .
$$

If the area is computed relative to the specific volume at which the equilibrium pressure crosses the Isotherm, Eq. 4.4 inolds for the shaded areas in Fig. 4.3.

Figure 4.4 shows a portion of the lithium P-V plane. The isotherms are from $2750^{\circ} \mathrm{C}$ to $3500^{\circ} \mathrm{C}$ in $50^{\circ} \mathrm{C}$ increments. The open square is the critical point. The dashed lines are the spirodal lines and the open circles 11e on the coexistence curve. The coexistence curve pecks at the critical point.

A wathSY 20 macro was written to plat the oquations for pressure, internal energy, and eritropy. A copy of the macro is avatleble 21 . 
Fig. 4.4. P-V diegren for lithium. The isotherms are at $50^{\circ} \mathrm{C}$ intervals. The deshed lines are the lsotherwal spinodals. The left isentropic spinodal passes through $(0.012,-100),(0.02,-50)$, and $(0.03,-20)$ and probebly does not reach the positive pressure reglon. Response to lsochoric hating will probebly be more isentrople than isothormal since there is Insufficient time for heat transfer.

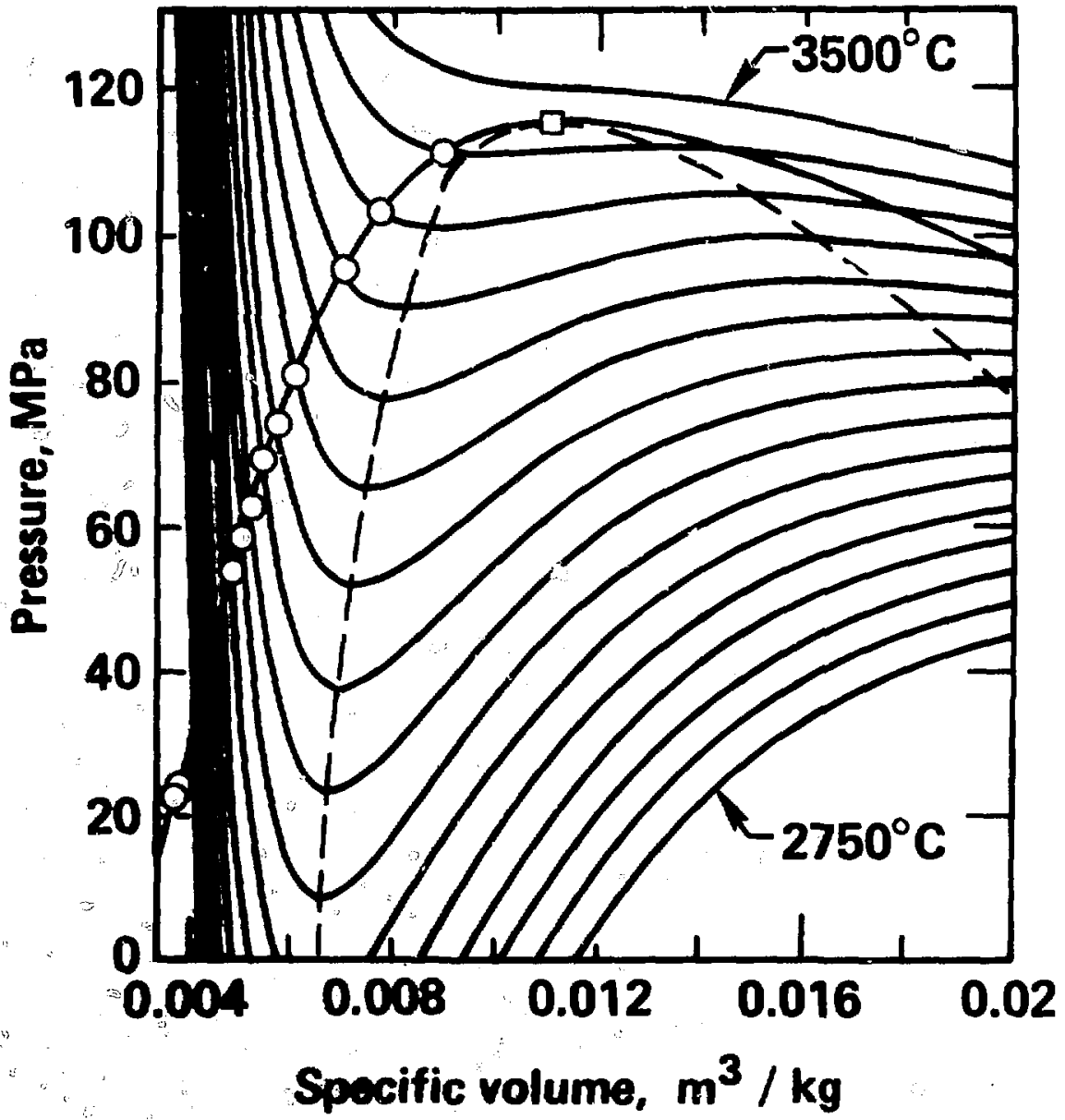


4.2. Two-Dimensional Lennard-Jonesium Phase Diegram

Two-dimensional molecular dynmics simulations will be used in Chapter Seven to test the (hydrodynemic) particle size modals. Two-dimensional simulations are used because they best simulate the quasi-two-dimensional expansion of e cylindrical jet, because thay are easliy ilagnosed graphically, and because they allow consideration of larger radius systems with linited computer resources. The properties of two dimensional Lennard Jones (LJ) materlal are well known, as is discussed in this section. This section also includes a comparison of the LJ equation of state data from several authors, construction of analytic fits to the data, and determination of the two "free" LJ constants for lithium.

A general equation for the $L J$ potential between two partlcles with interparticle separation variable, $r$, is

$$
\phi(r)=\left(\frac{E}{\xi-6}\right)=\left(\frac{E}{6}\right)^{6 /(E-6)}\left[\left(\frac{\sigma}{r}\right)^{E}-\left(\frac{\sigma}{r}\right)^{6}\right] .
$$

Most studies use $\xi$ in the 9 to 15 range; $\xi=12$ is used here. Then,

$$
\frac{\varphi(r)}{c}=4\left[\left(\frac{\sigma}{r}\right)^{12}-\left(\frac{\sigma}{r}\right)^{6}\right] .
$$

Since force is $(-2 \phi / \partial r)$,

$$
|F(r)| /\left(\frac{\varepsilon}{\sigma}\right)=24\left(\frac{\sigma}{r}\right)^{7}\left[2\left(\frac{\sigma}{r}\right)^{6}-1\right] .
$$

These functions are shown in Fig. 4.5. The potential is zero at $I=\sigma$ and $(-\varepsilon)$ at the bond rest length.

Barker ${ }^{22}$ has established a phase diagran for two-dimensional 
F1j. 4.5. The Lernard-Jones 6-12 interatomic potent Lal and assoclated rorce function truncated at $r=2.50$.
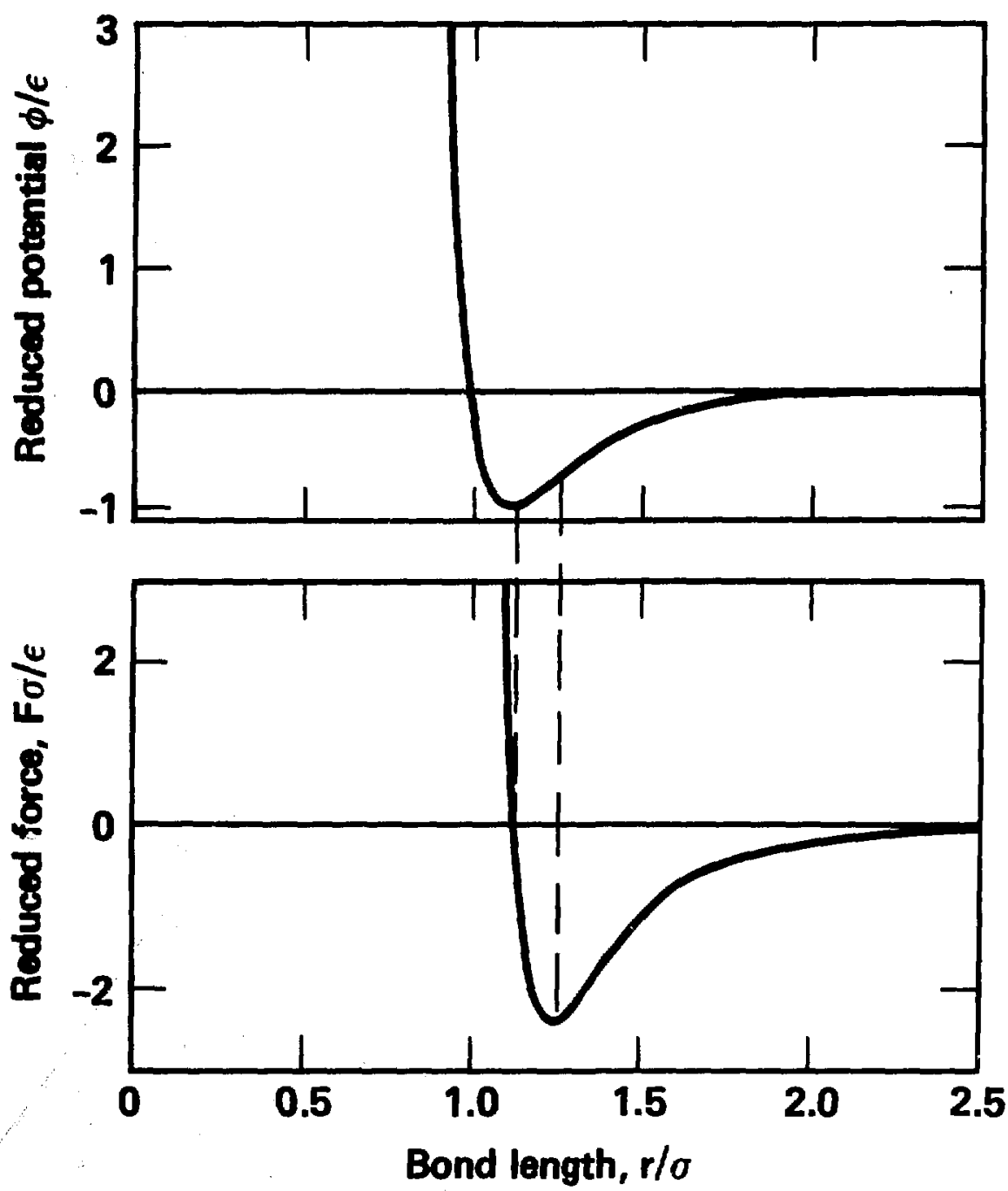
Lennard-Jonesium by using perturbation theory for the fluid phases and a self-consistent cell model for the solid phase. The phase boundarles are shown as solid lines in Flos. 4.6 and 4/7. Mnnte carlo calculations by Abrehan 23 have confirmed Barker's phase boundarles; Abrahan's Monte Carlo résults are shown as dashed lines in Fig 4.7. The dinensionless axes used in these floures $\left(\rho \sigma^{2}, \mathrm{kT} / \mathrm{c}\right.$, and $\mathrm{Po}^{2} / c$ ) allow use of the same phase diagrem for any combination of $\sigma$ and $c$. These constants can be set to matcin macroscopic properties of the chosen materlal.

Barker used the two-dinensional Monte Carlo data to generate Pade-type approximants of the form,

$$
\frac{P}{\rho k T}=\left[1+\sum_{1}^{5} f_{1} \rho^{1}\right] /\left[1+\sum_{1}^{4} g_{j} \rho^{j}\right] .
$$

As before, reduced variables are used, $P=P \sigma^{2} / c, \rho=\rho \sigma^{2}$, and $T=k T / \varepsilon$, with Boltzmann's constant $(k)$ set to 1.0. Barker calculated the values of the coefficients $\left(f_{1}\right.$ through $g_{4}$ ) for temperatures of $0.45,0.55,0.70$, and 1.0 (Table 4.2). The table also Indicates the calculated pressures for a density of 0.6 .

Barker's Monte Carlo pressure data 22 are compared to the Monte Carlo data of Henderson ${ }^{24}$, Phillips ${ }^{25}$, and Tsien ${ }^{26}$, and the molecular dynemics data of Fehder ${ }^{27}$ and Toxvaerd ${ }^{28}$ in Fig. 4.8. The data are plotted as compressibllity factor (P/pkT) contours on a $p-T$ diagram. Because Henderson's ${ }^{24}$ data are the most extensive, they are used as the basis for the contours. Where the other references indicate a shift in a contour, it is shom by dat.ted lines. The contours allow comparison of the references and 
Fig. 4.6. Phase diagram for two-dimensional. Lennard-Jonesium (density-temperature plane).

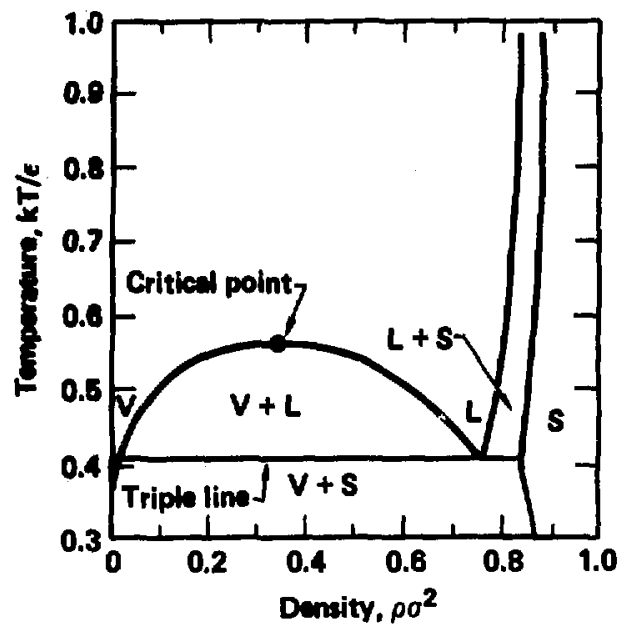

Fig. 4.7. Phase diagrem for two-dimensional I ennard-Jonesium (temperature-pressure plane). The solid lines are from perturbation theory ${ }^{22}$ and the dashed 11 nes are from Monte-Carlo calculations ${ }^{23}$.

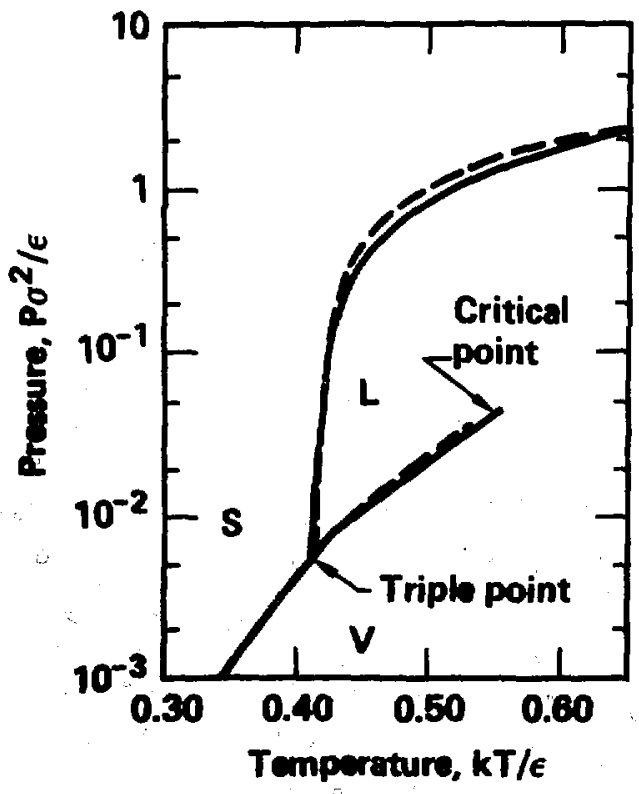


Table 4.2. Coefficients in Barker's pressure equation and his resulting pressures at $p=0.6$. The one Monte Carlo result at this density is also shown.

\section{Iemoarnture}

\begin{tabular}{|c|c|c|c|c|}
\hline & 0.45 & 0.55 & 0.70 & 1.0 \\
\hline$f_{1}$ & -10.5045 & -3.5966 & -2.7141 & -3.2086 \\
\hline$f_{2}$ & 20.7126 & 13.6709 & 16.2753 & 11.7969 \\
\hline$f_{3}$ & -13.0723 & -9.72 .82 & -37.2791 & -18.4305 \\
\hline$f_{4}$ & 2.5803 & 0.5640 & 36.7272 & 17.0168 \\
\hline$f_{5}$ & 0.0 & 0.0 & 0.0 & 0.0 \\
\hline$g_{1}$ & -2.8466 & 1.2134 & 0.0464 & -2.1356 \\
\hline$g_{2}$ & 10.9930 & 15.8445 & 12.0780 & 7.0799 \\
\hline$g_{3}$ & -11.1515 & 9.6091 & -14.2334 & -5.9005 \\
\hline$g_{4}$ & 0.0 & -40.0 & 0.0 & 0.0 \\
\hline$P(e q)$ & -0.108 & 0.132 & 0.356 & 0.936 \\
\hline$P(M C)$ & -0.100 & & & \\
\hline P/pkT(eq) & -0.399 & 0.401 & 0.847 & 1.56 \\
\hline P/pkT(MC) & -0.370 & & & \\
\hline
\end{tabular}


Fig. 4.8. Compressibility factor contours for 2-D Lennard-Jonesium. The solid lines are from Hendarson's data. Dotted lines are alternative contours from the Indicated references.

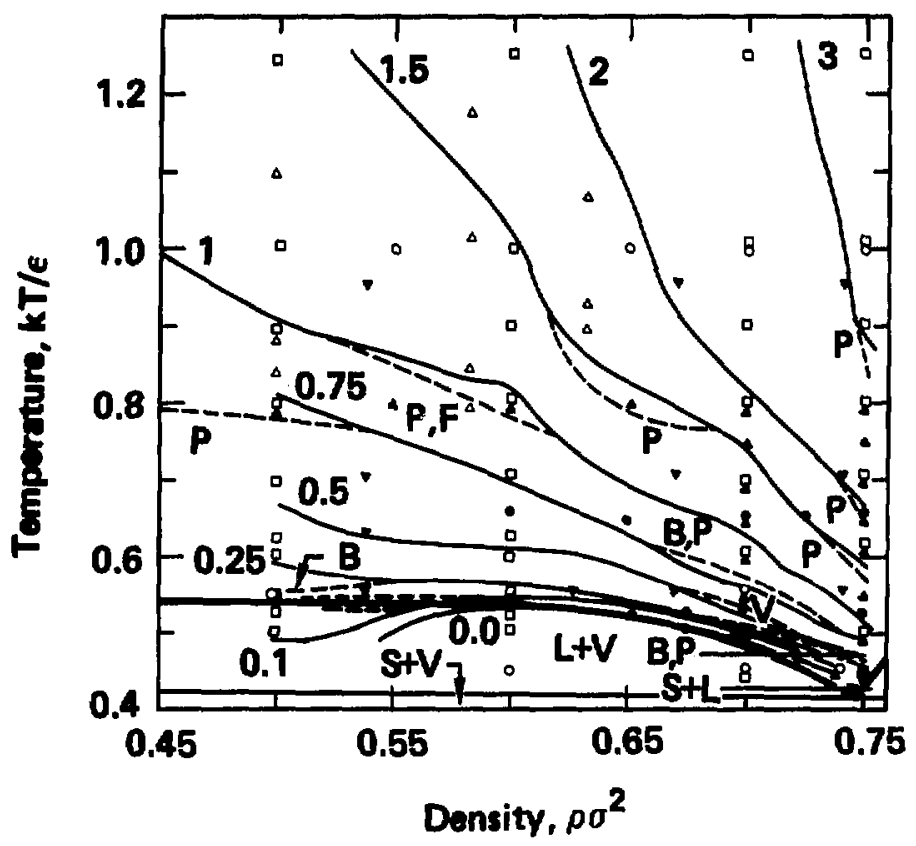

$\square$ Henderson

O Barker (B)

- Toxveard (V)

$\triangle$ Fehder (F)

A Phillips (P)

$\checkmark$ Tsien

$\rho_{\mathrm{c}} \sigma^{2} \sim 0.33$ 
visualization of the data. However, enalytic expressiens for the pressure as a function of temperature and density ware dorived frem the data, rather than the contours.

Points of overlep or inconsistency mong the references are summarized in Table 4.3. At high temperatures, the date are very consistent. At low temperature $(\sim 0.45)$ and hlgh density $(\sim 0.75)$, Barker's ${ }^{22}$ compressiblilty factor is lower then Henderson's ${ }^{24}$. The situation is reversed at a higher tmperature $(0.55)$ and lower density $(0.5)$. In general, Phillips' data ${ }^{25}$ egrese with Barker's rather than Henderson's when all three have comnon points. Phillips' compressibility factor is lower than Barker's at $(T=0.45 ; \rho=0.74)$, lower than Henderson's at $(T=0.8 ; \rho=0.7)$ and higher than Henderson's over a range of conditions $(T=0.6$; $\rho=0.7$ and 0.75$),(T=0.7 ; \rho=0.75)$ and $(T=0.8 ; \rho=0.5$ and $0.6)$. Fehder's data ${ }^{27}$ at $(\rho=0.5 ; T=0.84$ and 0.88$)$ are higher than Henderson's comparable data. Sone of Tsien's data ${ }^{26}$ are higher than expected from the contours generaled from the other data $(T=0.55,0.7$ and $0.95 ; \rho=0.74),(T=0.7 ; \rho=0.67)$ and $(T=0.95 ; \rho=0.54)$. However, other $T$ sien data $(T=0.7$; $\rho=0.54)$ and $(T=0.55 ; \rho=0.67)$ are lower than expected. At two points of overlap $(T=0.65 ; p=0.7$ and 0.75$)$, Toxvaerd's data $^{28}$ are five to ten parcent less than PhLllips' values, but PhIllips' data are five to ten percent higher then Henderson's in a nearby region. Toxvaerd's point at $(T=0.655 ; p=0.6)$ is only $\sim$ 80\% of the value interpolated from Henderson's data.

Barker's Monte Carlo data differ sionificently from his equation at low temperatures. Tsien's data point at $(T=0.55 ; p=0.67)$ is 
Table 4.3 Overlepping points and inconsistencies in the compressiblilty factor (P/pkT) data. Sources: H (Henderson ${ }^{24}$ ), $B$ (Barker $\left.{ }^{22}\right), P$ (Phillips $\left.{ }^{25}\right), V$ (Toxvand $\left.^{28}\right), F$ (Fehdor ${ }^{27}$ ), and $T$ (Tsien 26$)$.

\begin{tabular}{|c|c|c|c|c|c|c|c|}
\hline$T$ & p & H & $B(M C)$ & $\frac{\text { Source }}{B(\Delta q)}$ & $P$ & $v$ & $F$ \\
\hline $\begin{array}{l}0.45 \\
0.45 \\
0.444 \\
0.45 \\
0.45 \\
0.45 \\
0.444 \\
0.5 \\
0.5 \\
0.5 \\
0.525 \\
0.525 \\
0.55 \\
0.55 \\
0.55 \\
0.6 \\
0.6 \\
0.625 \\
0.625 \\
0.655 \\
0.7 \\
0.65 \\
0.654 \\
0.65 \\
0.651 \\
0.7 \\
0.7 \\
0.8 \\
0.838 \\
0.8 \\
0.8 \\
0.80 \\
0.9 \\
1.0 \\
1.0 \\
1.0\end{array}$ & $\begin{array}{l}0.6 \\
0.7 \\
0.7 \\
0.738 \\
0.739 \\
0.75 \\
0.75 \\
0.5 \\
0.7 \\
0.75 \\
0.4 \\
0.5 \\
0.5 \\
0.67 \\
0.7 \\
0.7 \\
0.75 \\
0.5 \\
0.6 \\
0.6 \\
0.6 \\
0.7 \\
0.7 \\
0.75 \\
0.75 \\
0.7 \\
0.75 \\
0.5 \\
0.4993 \\
0.6 \\
0.7 \\
0.4993 \\
0.5 \\
0.55 \\
0.7 \\
0.75 \\
0\end{array}$ & 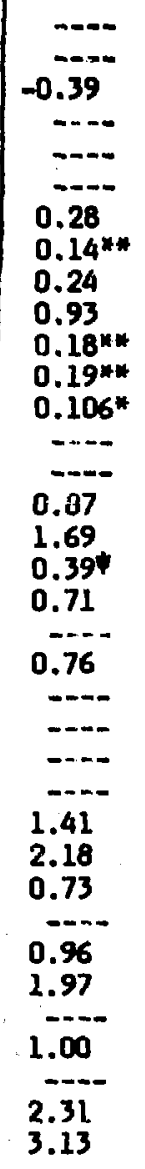 & 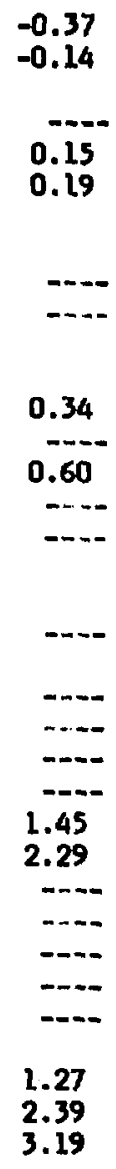 & 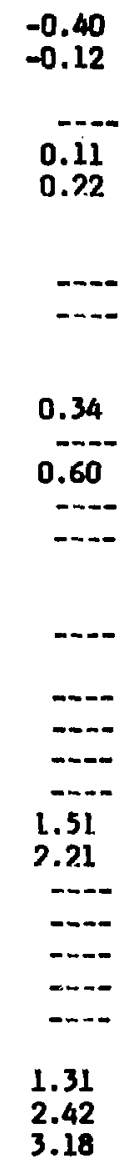 & $\begin{array}{l}: \\
0.03 \\
0.18 \\
0.13 \\
0.85 \\
\\
0.62 \\
0.97 \\
1.75\end{array}$ & $\begin{array}{l}0.59 \\
1.16 \\
1.91\end{array}$ & $\begin{array}{l}0.98 \\
1.08\end{array}$ \\
\hline
\end{tabular}


not betwen the values at brecketing densities. Stailiriy, Henderson's data point at $(T=0.55 ; \rho=0.5)$ does not lie batwen the values at bracketing temperaturas. Finally, Hendarson's compressibility factors at $(T=0.5 ; \rho=0.5)$ and $(T=0.525$; $\rho=0.4$ and 0.5 ) are not negative even though they probebly 11 e within the two phase liquid-vapor region.

The date polnts from the references are listad in Table 4.4 for the density range $0.45-0.76$ and the tomperature range $0.4-1.3$. Because Henderson's data are the most extensive, they were used to construct anslytic equations for the pressure in terms of the density and temperature for the T-p region investigated in the molecular dynamics experiments (described in Chapters Six and Seven). Pressure isntherms are shown in Fig. 4.9 and pressure lsochores are shown in Fig. 4.10. These figures also show Barker's equation in the same region. The specific heat at constant valume, $c_{v}$, is shown in Flg. 4.11 for three densities. The equations for the specific heat and the pressure 1sotherms and isochnres are shown in Table 4.5. As before, reduced units $\left(\rho \sigma^{2}, k T / \varepsilon, P \sigma^{2} / c, E / N c\right)$ are used in Table 4.5. The equations for $(2 P / \partial \rho)_{T}$ vs. $T$ (1sochores) from Henderson's data were generated by fitting a cublc equation in temperature to the $(\partial P / \partial \rho)_{T}$ data point.s individually evaluated from cubic fits of the pressure isotherms. This cumbersome procedure was used since data were needed at only a few densities but many temperatures.

The well depth, c, can be estimated from the heat of veporization. In three dimensions, bout 12 bonds sust be broken to free one atom, and each bond is shared by two atoms. Hence, the heat 
Table 4.4. Compressibllity factor data. Source codes are M (Barker's Monte Carlo), E (Barker's equation), H (Henderson), T (Tsien), $P$ (Phillips), $V$ (Toxveerd), and F (Fehder).

\begin{tabular}{|c|c|c|c|c|c|c|c|c|}
\hline & & & & anre & & & & \\
\hline$p$ & 0.444 & 0.45 & 0.5 & 0.523 & 0.525 & 0.527 & 0.55 & 0.6 \\
\hline 0.5 & -..... & $-0.59 E$ & $0.14 \mathrm{H}$ & $-\cdots--$ & $0.19 H$ & n...- & $\begin{array}{l}0.34 \mathrm{E} \\
0.34 \mathrm{M} \\
0.11 \mathrm{H}\end{array}$ & $0.34 \mathrm{H}$ \\
\hline 0.530 & $-\cdots$ & $-0.32 E$ & ---- & $-\cdots--$ & ---- & $-\cdots-$ & $\begin{array}{l}0.35 E \\
0.08 T\end{array}$ & \\
\hline 0.6 & $-\cdots$ & $\begin{array}{l}-0.40 E \\
-0.37 \mathrm{M}\end{array}$ & $-0.02 \mathrm{H}$ & $-\cdots$ & $-0.02 \mathrm{H}$ & $-\cdots$ & $\begin{array}{l}0.40 \mathrm{E} \\
0.22 \mathrm{H}\end{array}$ & $0.39 \mathrm{H}$ \\
\hline 0.627 & $\cdots$ & $-0.34 E$ & $\cdots$ & -..-- & $-\cdots$ & $\cdots$ & $\begin{array}{l}0.43 E \\
0.30 \mathrm{~T}\end{array}$ & \\
\hline 0.65 & $\cdots$ & $-0.28 E$ & $\ldots$ & ---- & $0.12 \mathrm{~V}$ & 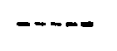 & $0.47 E$ & \\
\hline 0.67 & -...- & $-0.23 E$ & $\cdots$ & $-\cdots-$ & $\cdots$ & --.- & $\begin{array}{l}0.51 E \\
0.231\end{array}$ & \\
\hline 0.675 & ---.- & $-0.2 .1 \mathrm{E}$ & $-\cdots$ & $-\cdots+$ & $0.25 \mathrm{~V}$ & -...- & $0.52 E$ & \\
\hline 0.696 & $-\cdots$ & $-0.14 E$ & $0.07 \mathrm{P}$ & -.-- & -... & --.-- & $0.56 E$ & \\
\hline 0.7 & $-0.39 H$ & $\begin{array}{l}-0.12 E \\
-0.14 \mathrm{M}\end{array}$ & $\begin{array}{l}0.24 \mathrm{H} \\
0.13 P\end{array}$ & $m-\infty$ & $-\cdots$ & $0.46 \mathrm{~V}$ & $\begin{array}{l}0.60 \mathrm{E} \\
0.60 \mathrm{M} \\
0.62 \mathrm{P}\end{array}$ & $\begin{array}{l}0.87 H \\
0.97 P\end{array}$ \\
\hline 0.758 & $-\cdots$ & $\begin{array}{l}0.10 E \\
0.03 P\end{array}$ & $-\cdots$ & -...- &..-- & $-\cdots$ & $0.81 E$ & \\
\hline 0.739 & 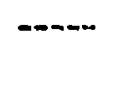 & $\begin{array}{l}0.11 E \\
0.15 N\end{array}$ & -...- & -...- & -... & $--\cdots$ & $0.82 E$ & \\
\hline 0.741 & $\cdots$ & $0.12 E$ & ---- & $\cdots$ & -.... & $-\cdots$ & $\begin{array}{l}0.84 \mathrm{E} \\
1.28 \mathrm{~T}\end{array}$ & \\
\hline 0.75 & 0.204 & $\begin{array}{l}0.22 E \\
0.194 \\
0.189\end{array}$ & $\begin{array}{l}0.93 H \\
0.85 P\end{array}$ & $1.22 \mathrm{~V}$ & -...- & $---\infty$ & $\begin{array}{l}0.93 E \\
1.26 \mathrm{P}\end{array}$ & $\begin{array}{l}1.694 \\
1.75 P\end{array}$ \\
\hline
\end{tabular}


Toble 4.4 (Continued). Compressibility fector L. Sorron cedes ane $M$ (Barker's Monte Carlo), E (Barker's equation), H (Hendereen), $T$ (Tslen), P (Phillips), $V$ (Toxveord), and F (Fehder):

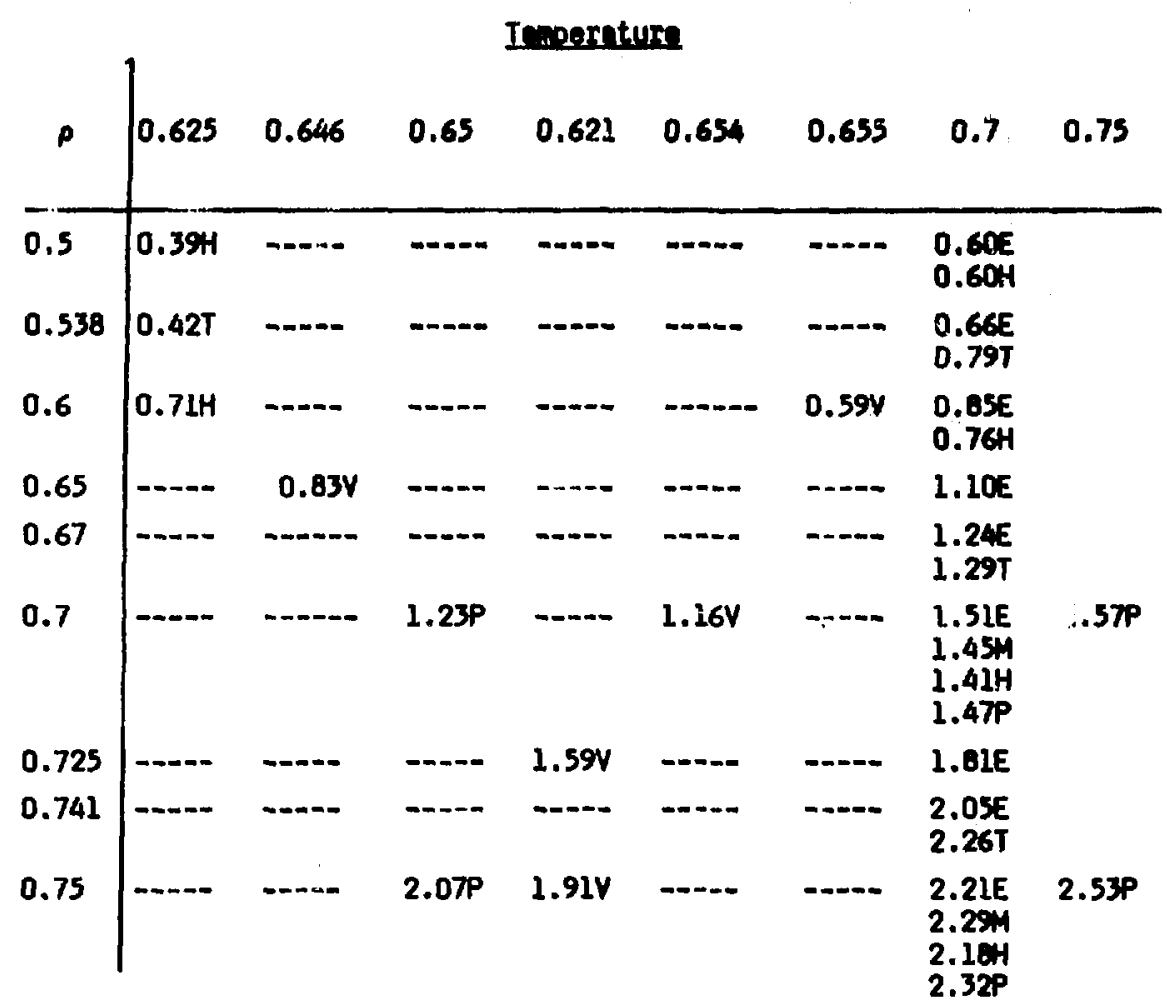


Table 4.4 (Continued). Compressibility factor data. Source codes are M (Barker's Monte Carlo), E (Barker's equation), H (Henderson), $T$ (Tsien), P (Philllips), $V$ (Toxveerd), and $F$ (Fohder).

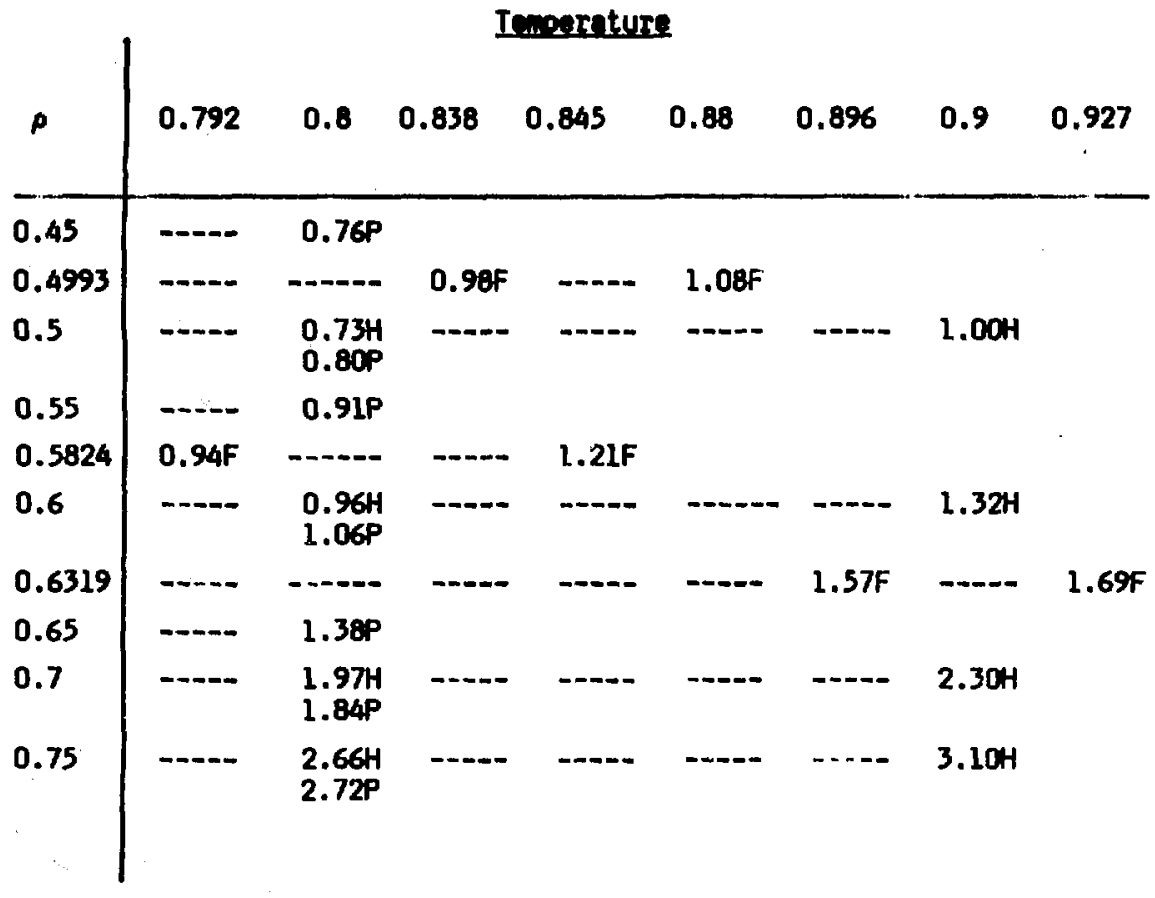


Table 4.4 (Continued). Compressiblifty fector date. Source codse are $M$ (Barker's Monte Carlo), E (Barker's equation), H (Findsrson), T (Tsien), P (Phillips), $V$ (Toxveerd), and F (Fohder).

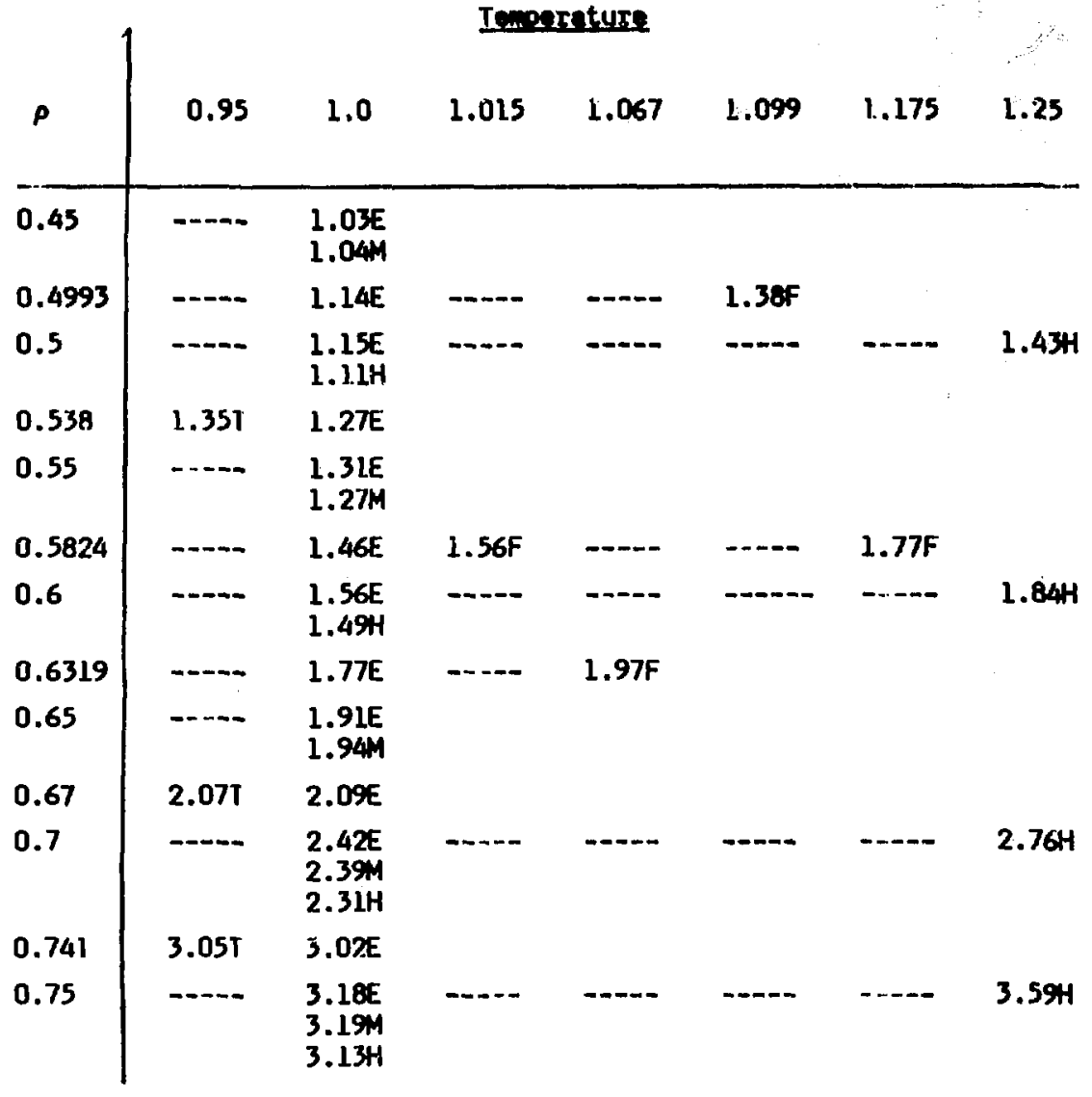


F1g. 4.9. Pressure 1sotherms from Barker's equation and Henderson's Monte Carlo data. Barker's equation is a Pade approximant

(Eq. 4.17). Hendarson's data were used to generate cublc 1sotherms. Higher order flts lead to excessive fluctuations in the derivative, $(a P / \partial p)_{T}$.

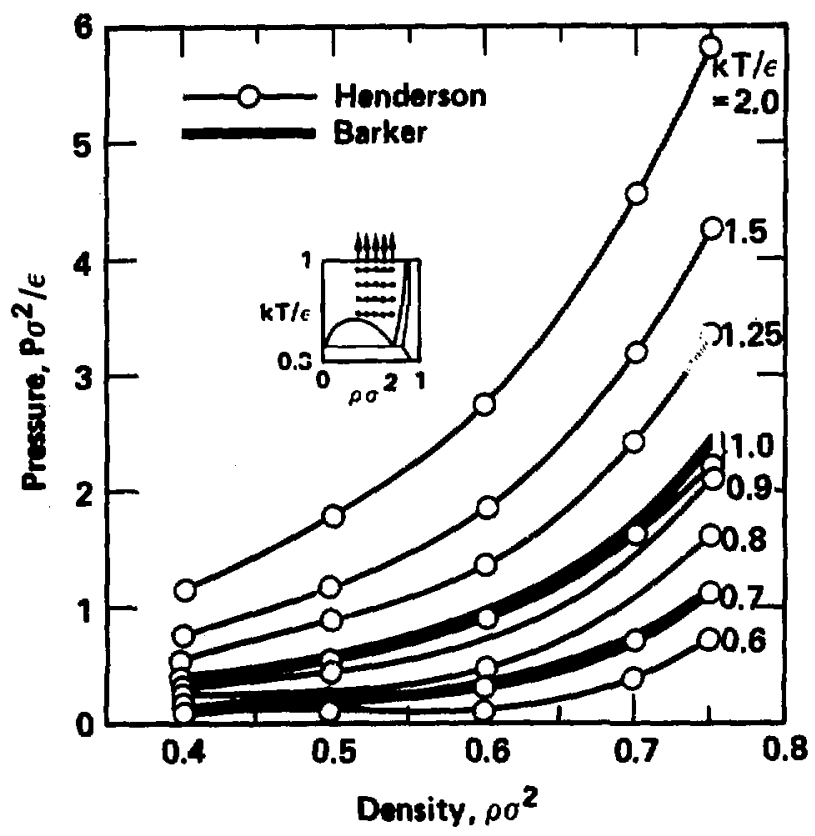


Fig. 4.10. Pressure 1sochores from Barker's equation and Honderson's Monte Carlo data. The single 1sochore from Barker we obtalned by fitting quadratic to the valuse of Barkor's lsothormal equations at $\rho=0.6$. A cublc fit was unsuiteble beceuse it produced excessive

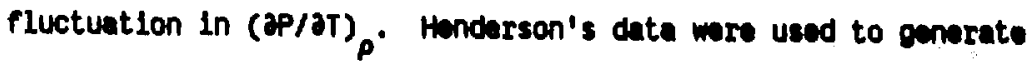
quedratic equations for the isochores.

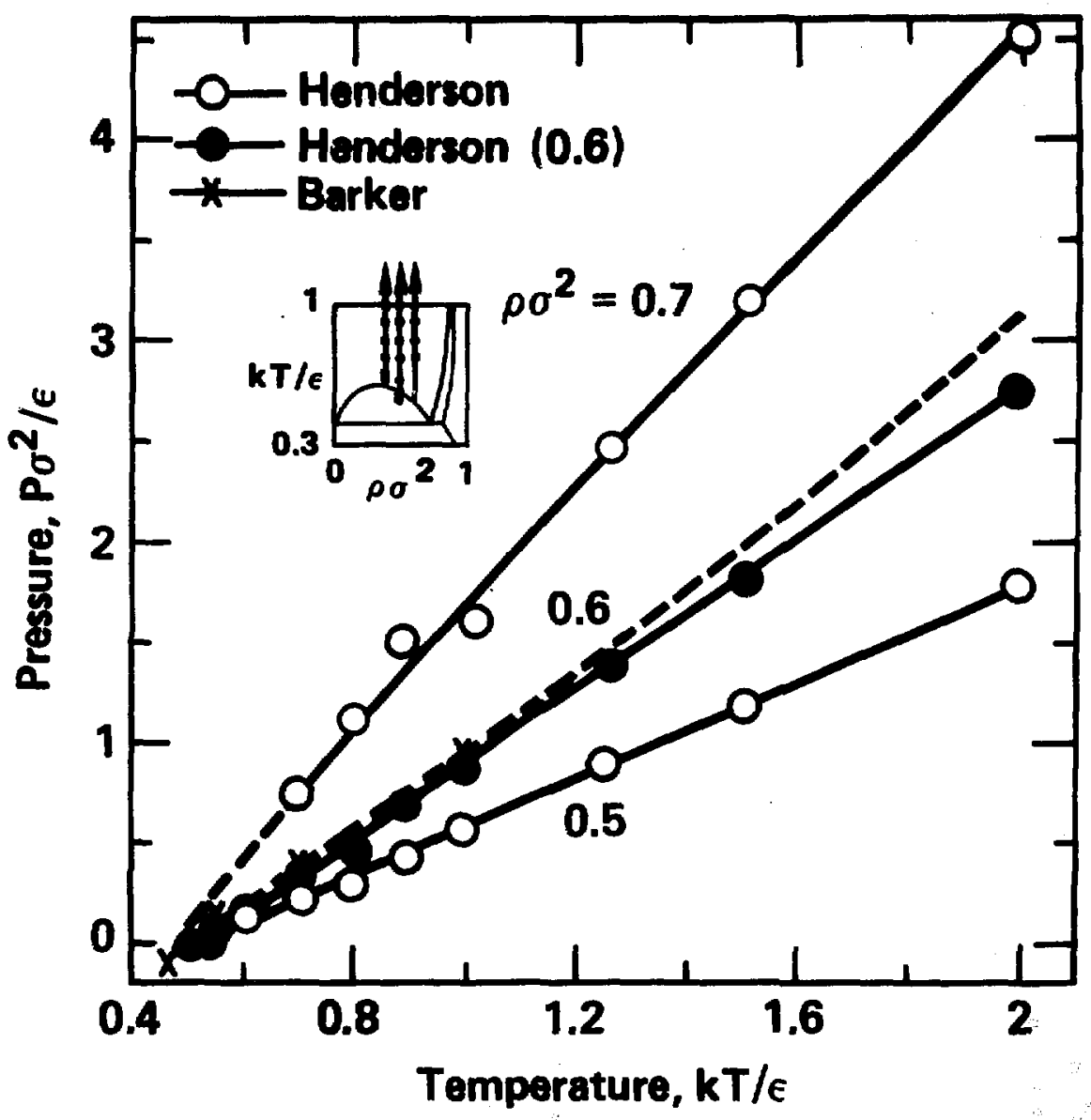


Fig. 4.11. Specific heat from Henderson's Monte Carlo energy data. The curves are based on quadratic fits to the energies at $\rho=0.5$ and 0.6, and on a cublc fit to the energies at $p=0.7$. The curves Include both the kinetlc and the configurational contributions.

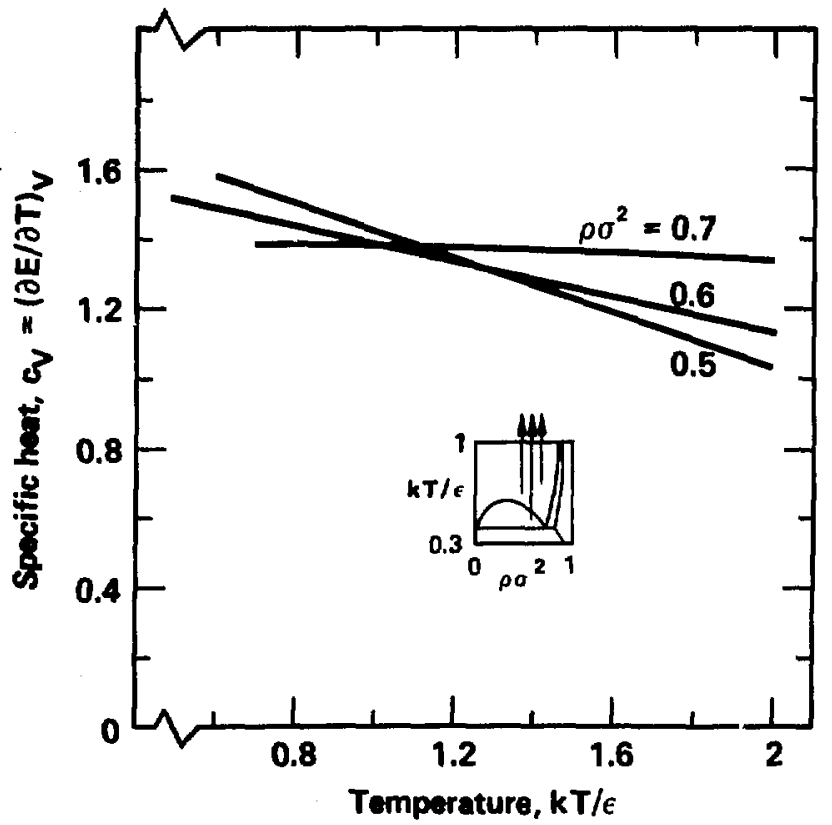


Table 4.5. Equations dorived from Barker's pressure equation and Henderson's Monte Carlo data. Both kinatic and configurational contributions are included.

$E= \begin{cases}-0.9221+1.8171 T-0.19740 T^{2} & , p=0.5 \\ -0.7644+1.6418 T-0.12772 T^{2} & , p=0.6 \\ -0.9661+1.3799 T+0.01102 T^{2}-0.00721 T^{3} & , p=0.7\end{cases}$

$c_{V}=\left(\frac{\partial F}{\partial T}\right)_{V}= \begin{cases}1.0171-0.39480 T & , p=0.5 \\ 1.6418-0.25544 T & p=0.6 \\ 1.3799+0.02204 T-0.021631 T^{2} & , p=0.7\end{cases}$

$\begin{array}{ll}P(1)_{p} \\ \text { Henderson }\end{array}= \begin{cases}-0.66563+1.25280 T-0.01824 T^{2} & , p=0.5 \\ -0.99998+1.91659 T-0.01631 T^{2} & , p=0.6 \\ -1.61384+3.48080 T-0.20996 T^{2} & , p=0.7\end{cases}$

$P\left(T_{P}\right.$

Barker

$$
=-0.81032+1.51861 T+0.22655 T^{2} \quad, p=0.6
$$

$\begin{aligned} & (\partial P / \partial T)_{A} \\ & \text { Henderson }\end{aligned}=\left\{\begin{array}{l}1.25280-0.03648 T \\ 1.91659=0.03262 T \\ 3.48088=0.41992 T\end{array}\right.$

,$p=0.5$

, $\rho=0.6$

$p=0.7$

$(\partial P / \partial T)_{P}=1.51861+0.45309 T$

, $\rho=0.6$

Barker

$\begin{array}{ll}(\partial P / \partial \rho)_{T} \\ \text { Henderson }\end{array}= \begin{cases}-4.66013+8.49535 T-2.3956 T^{2}+0.58100 T^{3} & , \rho=0.5 \\ -6.66520+15.2262 T-4.5570 T^{2}+0.95618 T^{3} & ; \rho=0.6 \\ 0.92390+0.87770 T+13.540 T^{2}-4.21899 T^{3} & , \rho=0.7\end{cases}$

$\underset{\text { Barker }}{(\partial P / \partial \rho)_{T}}=k T\left[\left[\left(1+\sum_{1}^{5}(1+1) f_{1} \rho^{1}\right) /\left(1+\sum_{1}^{4} g_{j} \rho^{j}\right)\right]-\right.$

$$
\left.\left[\left(\rho+\sum_{1}^{5} f_{1} \rho^{1+1}\right)\left(\sum_{1}^{4} j g_{j} \rho^{j-1}\right) /\left(1+\sum_{1}^{4} g_{j} \rho^{j}\right)^{2}\right]\right\}
$$

( $f_{1}$ through $g_{4}$ are listed in Table 4.2) 
of veporization is obout six times the well depth. If just-melted Iithium is taken as the reference point, the heat requitred for veporization is the cohosive energy $(23.03 \mathrm{~mJ} / \mathrm{kg})$ less the heat required to convert room temperature IIthium to Just-melted lithium (1.03 $\mathrm{mJ} / \mathrm{kg})$.

$$
6 c=(23-1.03) \mathrm{MJ} / \mathrm{kg}
$$

or

$$
c=3.667 \frac{\mathrm{mg}}{\mathrm{kg}} / 8.679 \times 10^{25} \frac{\mathrm{tton}}{\mathrm{kg}}=4.225 \times 10^{-20} \mathrm{~J} / \mathrm{atom} .
$$

The zero-potential interatomic separation, $\sigma$, can be found from the llquid density as follows. First, a hexagonal close packed (HCP) structure with a fixed layer spacing corresponding to the dansity of 1 ithium at $500^{\circ} \mathrm{C}$ is assumed. The HCP lattlce has a two-atom unit cell that contains portions of four atoms on each of two base planes, and one atom on an intermediate plane (FIg. 4.12). The Interwadiate plane atom and three base plane atoms form a tetrahedron. The HCP lattice constant, $I_{L}$, is twice the atomic radlus, and the layer spacing is

$$
r_{h}=\left\{r_{L}^{2}-\left[(2 / 3)(\sqrt{3 / 2}) r_{L}\right]^{2}\right\}^{1 / 2}=(2 / 3)^{1 / 2} r_{L} .
$$

Hence, the cell volune per atom is

$$
\begin{aligned}
V & =r_{L}\left[(\sqrt{3} / 2) r_{L}\right]\left[2(\sqrt{2} / \sqrt{3}) r_{L}\right] / 2 \text { atoms } \\
& =(1 / \sqrt{2}) r_{L}^{3} .
\end{aligned}
$$

If the density is decreased by increasing the base bond lengths while maintalning the layer spacing (tetrahodron height),

$$
v=\left[(\sqrt{3} / 2) r_{L}^{2}\right]\left(2 r_{h}\right) / 2=(\sqrt{3} / 2) r_{L}^{2} r_{h}
$$


Fig. 4.12. Unit cell for a hexagonal-close-packed lattice. In the two-dimensional nodel of $500^{\circ} \mathrm{C}$ lithlum, the undistarted three dimensional lattice is matched to the known lithium density. The unit cell is shown with heavy lines. Atoms in the unit cell are indicated with heavy symbols.

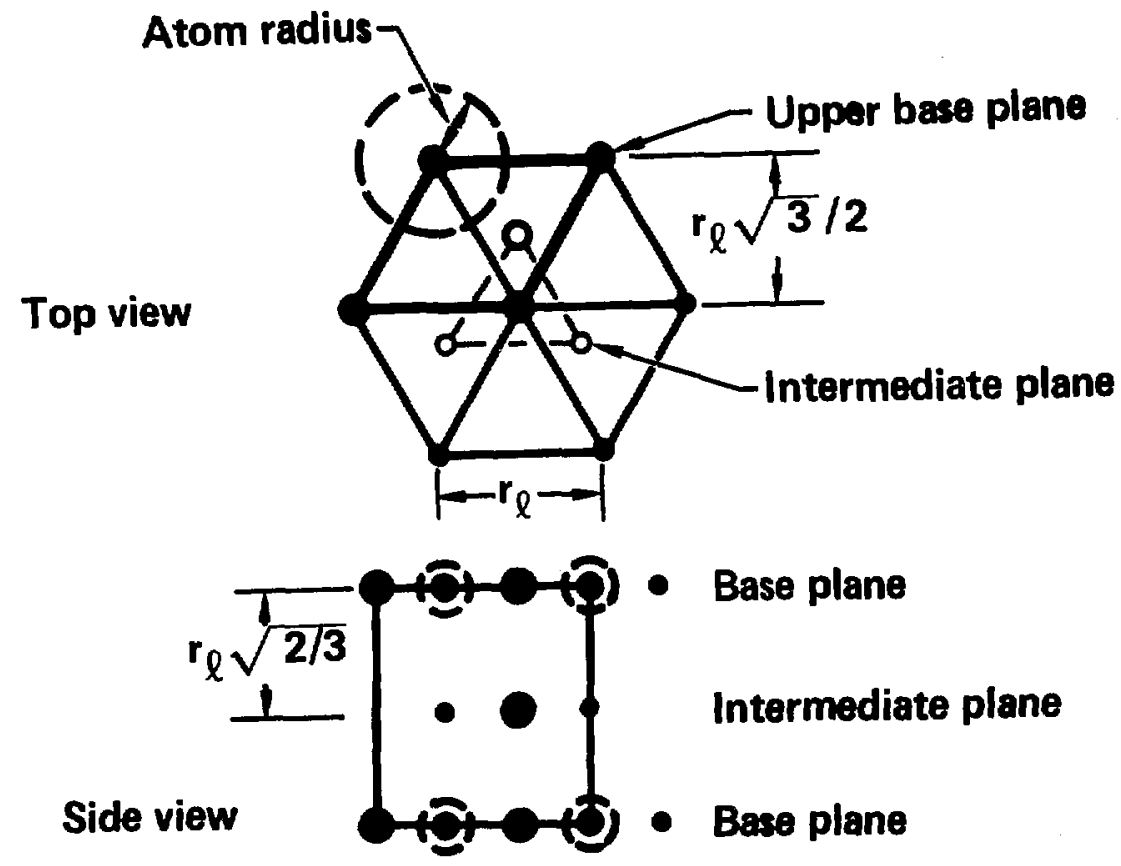


setting $r_{h}=(2 / 3)^{1 / 2} r_{L}$ at $500^{\circ} \mathrm{C}$ lithium density,

$$
\begin{aligned}
& V=\left[\left(8.679 \times 10^{25} \text { atom } / \mathrm{kg}\right)\left(485 \mathrm{~kg} / \mathrm{m}^{3}\right)\right]^{-1}, \\
& r_{h}=2.633 \times 10^{-10} \mathrm{~m}
\end{aligned}
$$

end

$$
r_{0}=r_{L}=3.225 \times 10^{-10_{m}}
$$

At the rest length, $r_{0}$, the potential is minimum and $a_{\phi} / \partial r=0$,

or,

$$
(2 \phi / \partial r)_{r=r_{0}}=0=4 \varepsilon\left[-12 \sigma^{12} / r_{0}^{13}+6 \sigma^{6} / r_{0}^{7}\right] \text {. }
$$

$$
\sigma=(2)^{-1 / 6} r_{0}=2.873 \times 10^{-10_{m}} .
$$

To further simplify the molecular dynamics calculations, it is desirable to use non-dimensionalized quantities to eliminate as many Irrelevant constants as possible. Accordingly, the distance was measured in units of $\sigma$, the energy in units of $\varepsilon$, the mass in units of the lithium atomic mass, and temperature in units of $\varepsilon / k$. These units were used to determine the scales for time, velocity, temperature, force, density and pressure (Table 4.6). In addition, the LJ potential was truncated at $r_{t}=40$ (in early calculations) and $r_{t}=2.50$ (in later calculations). Truncation saves time by Ionoring the large number of very weak bonds. The change in truncation procedure had little effect on the atonlc motion. This is epperent from the lattlce energy per atom for various truncation distences end densities (Fig. 4.13). Therefore, the choice of truncation distence is not critical. Shifting the potential upward by $\phi\left(r_{t}\right)$ moothe the potential by elininating step jumps in lattice 
Table 4.6. SI values of the reduced units.

\section{Quntity}

dislance

energy

mass

velocity

time

temperature

force

densily (2-D)

pressure (2-D)

\section{SI vilue for $500^{\circ} \mathrm{C}$ lithive}

$2.873 \times 10^{-10} \mathrm{m(c)}$

$4.225 \times 10^{-20} \mathrm{~J}(\mathrm{c})$

$1.152 \times 10^{-26} \mathrm{~kg}\left(1 / \mathrm{N}_{\mathrm{m}}\right)$

$1915 \mathrm{~m} / \mathrm{s}\left([\mathrm{c} / \mathrm{m}]^{1 / 2}\right)$

$0.1500 \mathrm{ps}\left(\sigma / v=\sigma(m / c)^{1 / 2}\right)$

$3061 \mathrm{~K}\left(\mathrm{mv}^{2} / \mathrm{k}=\mathrm{c} / \mathrm{k}\right)$

$1.471 \times 10^{-10} \mathrm{~N}(\varepsilon / \sigma)$

$1.212 \times 10^{19}$ atom $/ \mathrm{m}^{2}\left(\sigma^{-2}\right)$

$0.5119 \mathrm{~Pa}\left(c / \sigma^{2}\right)$ 
Fig. 4.13. Lattice onergy per atom for several variations of the Lennard-Jones 6-12 potential. The curve labels indicate the truncation distance. The "shifted" curve indicates a potential that has been slightly increased to elininate a stop increase in potential at the truncation distence.

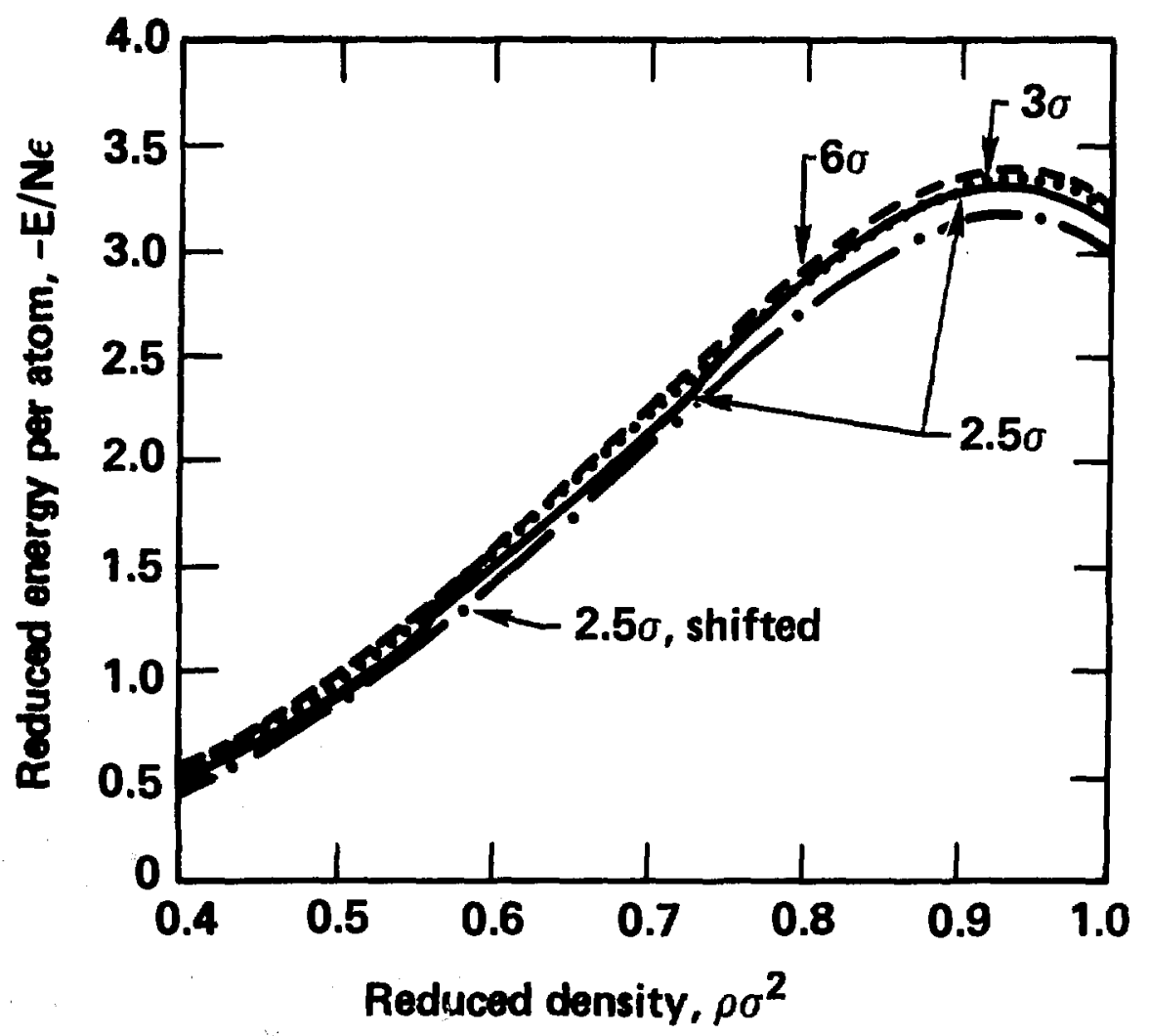


$-55-$

energy at densities that introduce additional atoms within renes of the potential. This shifted potential was used in pRof. 29.

The potential and force functions for the simplified LJ potential

are

$$
\begin{array}{ll}
\phi(r)= \begin{cases}4 r^{-6}\left(r^{-6}-1\right) & , I \leq r_{t} \\
0 & , r>r_{t}\end{cases} \\
\vec{F}_{1 j}=\frac{\vec{X}_{1}-\vec{X}_{1}}{r} \cdot \begin{cases}24 r^{-7}\left(2 r^{-6}-1\right) & , r \leq r_{t} \\
0 & , r>r_{t}\end{cases}
\end{array}
$$

(4.26)

where $r_{t}$ is the truncation distance. 
4.3. Thernodynaic Paths Followed on the P-V Diagram During and After Isochoric Heating

Som insight into the liquid breakup can be obtained by examining the pressure-volum dicigren for 1ithium. Floure 4.14 is a P-V diagrem for IIthium constructed using Young's equation of state (Section 4.1). The floure shows only a portion of the subcooled liquid region. The saturated liquid line is very shallow in thls density range; it is almost Indistingulatibble from the ebscissa. If the plot is extended far to the right, the saturated liquid line eventually rises to the critical pressure of $114 \mathrm{MPa}$ at $0.011 \mathrm{~m}^{3} / \mathrm{kg}\left(91 \mathrm{~kg} / \mathrm{m}^{3}\right)$.

The initial condition, before the fusion pulse, is on the saturated liquid line at $500^{\circ} \mathrm{C}\left(485 \mathrm{~kg} / \mathrm{m}^{3}\right)$. The fusion neutrons isochorically add a maximum of $800 \mathrm{~kJ} / \mathrm{kg}$ (HYLIFE inner jet inner edge). The liquid temperature and pressure quickly rise to $735^{\circ} \mathrm{C}$ and $300 \mathrm{Mpa}$ with no density change. As the relief wave moves into the jet, en adicbatic (isentropic) expansion begins. In an adiabatic expension, energy is conserved, although some of the internal energy cen be converted to kinetic and surface energies. During expansion to the saturation line (and $P=P_{\text {sat }} \sim 0$ ), the liquid temperature falls to $695^{\circ} \mathrm{C}$. During the expension, the internal eneruy falls by $-16 \mathrm{~kJ} / \mathrm{kg}$ from the value after the lsochoric heating. Use of Eqs. 2.3 and 2.4 shows that $\sim 15.2 \mathrm{~kJ} / \mathrm{kg}$ of kinetic energy is produced by the tensile wave, without considering any phase change. Hence, most of the "lost" internal snergy is converted to kinetic energy. The expension cen be continued into the two phase liquid-vepor region on the P-V diegrem to bound the veporization contribution (F10. 4.15). After the isentrope enters this two phase region, it 
Fig. 4.14. P-V diegrem of the heating and initiel axpenaien of a HYIFE Innor jat.

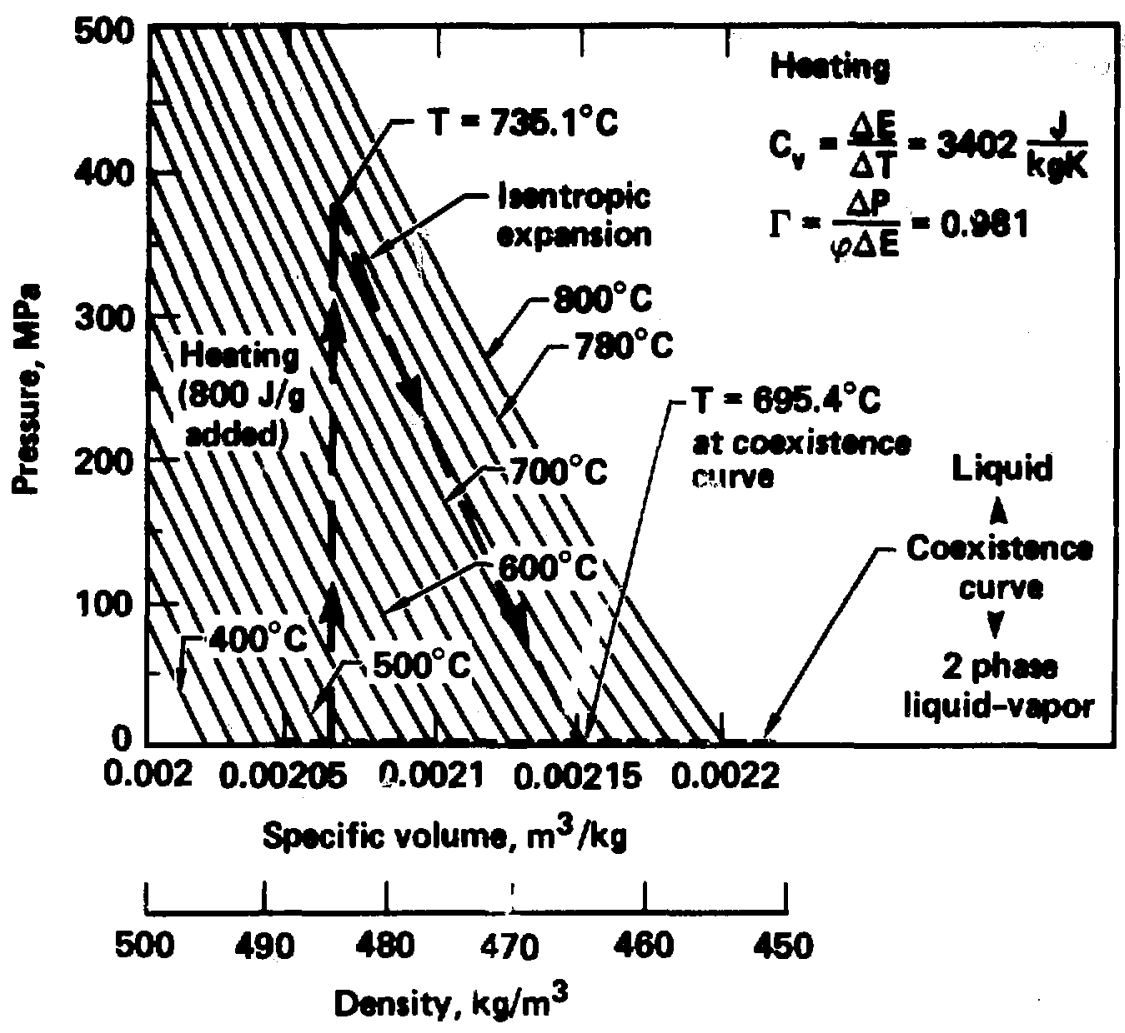


continues to expand to lower, oven negative, pressures. The isentrope Is not continued beyond the isentropic spinodal line where it has zero slope since the fluid is then unstable to phase separation back to the spinodai, probebly on a sub-nanosecond time scale. Batween the coexistence curve and the spinodal, the fluid is metastable, and it oventually separates into saturated liquid and vapor phases (on the coexistence curve) by the sequential processes of nucleation and growth or by veporization from the free surfaces if hydrodynamic fragmentation is faster than nucleation and growth.

If the isentropic expansion continued all the way to the spinodal, the intersection of the isentrope and spinodal would be as shown in the lower right corner of Fig. 4.15. The specific volume would increase by $77 \%$; and the vapor volume would be $\sim 0.0016 \mathrm{~m}^{3} / \mathrm{kg}-11$ quid. If the vepor were in equilibrium with $735^{\circ} \mathrm{C}$ liquid, the vapor pressure would be $\sim 120 \mathrm{~Pa}(0.9$ torr) and the vapor specific volume $\sim 969 \pi^{3} / \mathrm{kg}$-vapor. The quotient of the two specific volumes is $\sim 1.6 \times 10^{-7}$ (160 $\mu g$-vapor/ kg-liquid), an extrenely low veporization fraction. Since vaporization requires $20.7 \mathrm{~mJ} / \mathrm{kg}$-vapor (23.0 $e_{\mathrm{coh}}-1.0 \mathrm{H}_{\text {meli }}-1.3$ specific heat), only $3.4 \mathrm{~J} / \mathrm{kg}$-liquid are used for vaporization. Th1s is about $\$ 400$ times less energy than the kinetic energy produced by the expansion; trierefore, vaporlzation doos not uillize sufficient energy to drive the expansion. Further, the $77 x$ expension required before spinodal decomposition can begin would probably cause fregnentation hydrodynamically. Phase separation from the spinodel will eventually produce saturated liquid plus a mall mount of returated vepor (at sbout the seme temperature as the Intersection of the leantrope and the saturation line: $695^{\circ} \mathrm{C}$ in the 
Fig. 4.15. P.V diagraw showing isentropic expension to the isentricic spinodal line.

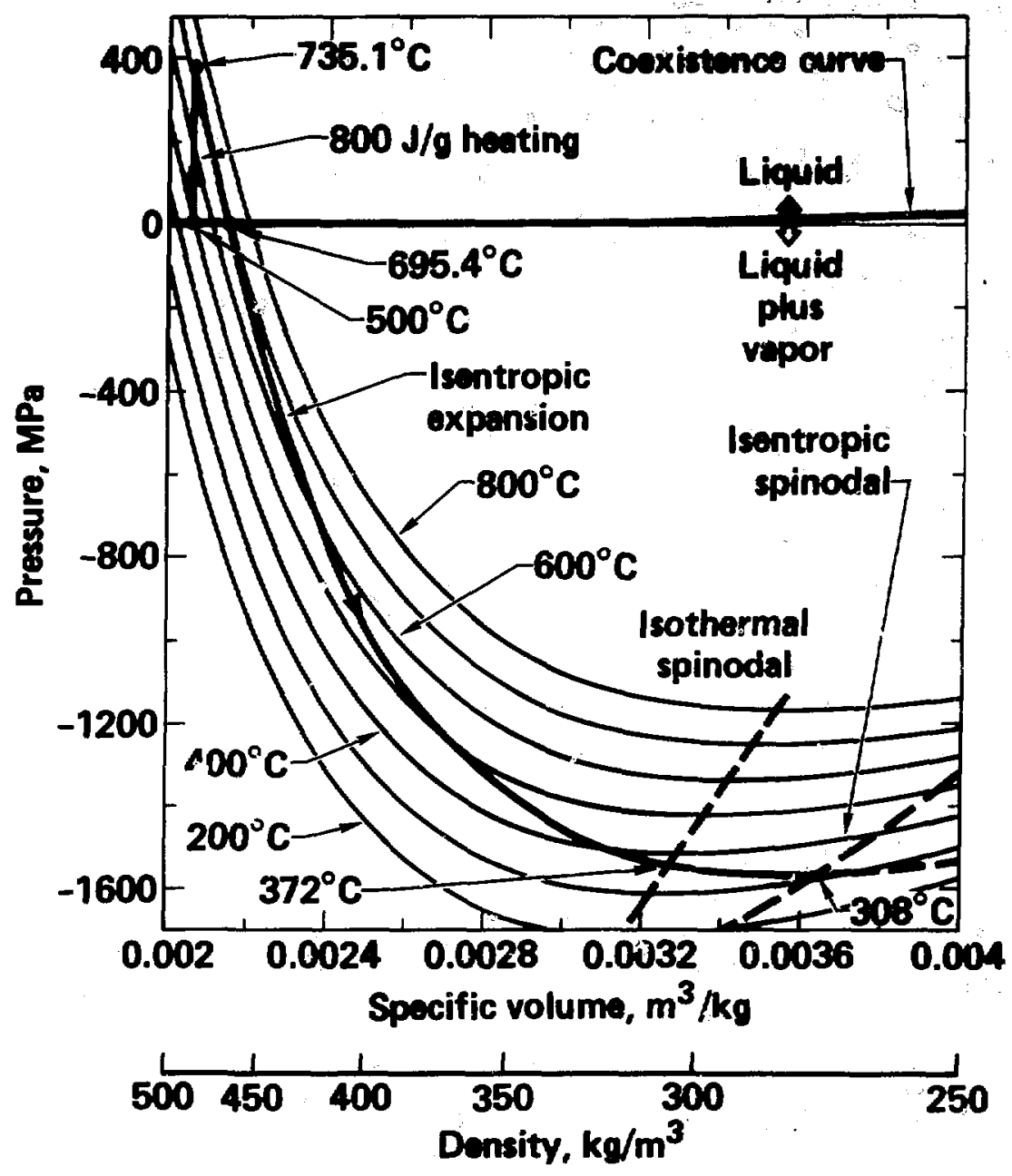


$-60-$

example).

By using the MTHSY macro end Young's equation of state, tharmodynamic valuas were calculated for several states during the hating and expansion (Tebie 4.7 ).

The discussion of phase change as a fragmentation mochanism wi.11 be concluded in Section 4.6, following a more datailed review of spinodal decomposition and of nucleation and growth In Sections 4.4 and 4.5 . 
Table 4.7. Thermodynamic values of the inner HMIFE jats bufore and after heating. These values assum a homogeneous expension without phase separation, and hence surface energy has been ienored.

\begin{tabular}{|c|c|c|c|c|}
\hline & $\begin{array}{c}\text { Initial } \\
\text { Condition }\end{array}$ & $\begin{array}{l}\text { After } \\
\text { Henting }\end{array}$ & $\begin{array}{l}\text { After Expension } \\
\text { to Cooxistence } \\
\text { Cury } \\
\end{array}$ & $\begin{array}{l}\text { After } \\
\text { Expension } \\
\text { to siningdil }\end{array}$ \\
\hline$T\left({ }^{\circ} C\right)$ & 500 & 735.1 & 695.4 & 300 \\
\hline$p\left(n g / m^{3}\right)$ & 484 & 484 & 465 & 274.2 \\
\hline$v\left(m^{3} / \mathrm{kg}\right)$ & 0.00206 & 0.00206 & 0.00215 & 0.00365 \\
\hline$P(M P a)$ & 0.000685 & 380 & 0.00337 & -1559 \\
\hline$E(k J / k g)$ & 2350 & 3149 & 3133 & 4935 \\
\hline$A(k J / k g)$ & -2988 & -4723 & -4429 & 390 \\
\hline$s(J / k g \cdot K)$ & 6905 & 7809 & 7809 & 7809 \\
\hline
\end{tabular}




\subsection{Splnodal Decomposition}

Spinodal decomposition is an extensively researched process. A 1902 literature soarch revealed over 500 publications on the subject. Perhaps the most stralghtforward sumary is contained in Cahn's 1967 Institute of Motals Lecture ${ }^{30}$ wich also cites many references. Reld ${ }^{31}$ has also published a very clear summary art.icle.

The concept of spinodal decomposition dates back to Gibbs in 1871. The concept is usually applied to a multi-component system with concentration being the independent varlabie. However, it is equally appllcable to a single component system with specific volume as the independent variable. The spinodal line is also called the limit of metastability, the superheat limit, and the cavitation limit.

Basically, spinodal decomposition is possible whenever the Gibbs free energy curve has a negative second derivative (Flg. 4.16). For a one-comporient fluld, this implies (JP/JV) $>0$. Consider a small separation into two equal masses of a composition between the spinodes (point 3 in Fig. 4.16). To conserve volume, the abscissa values (specific volumes) of the two constituents (points 2 and 4) must be equidistent from the original composition. Because of the negative second derivative, the decrease of free energy on the left side is larger then the increase on the right side. Since the overall free energy drops, the separation is energetically preferred. Separation continues until the energetics are no longer favarable; 1.e. to the equilibrium lines (points 1 and 5 ).

Cahn has applied the diffusion equation to spinodal decomposition In a two component system (components $A$ and B). 
Fig. 4.16. In spinodal decomposition, there is no energy barrier to small phese separations.

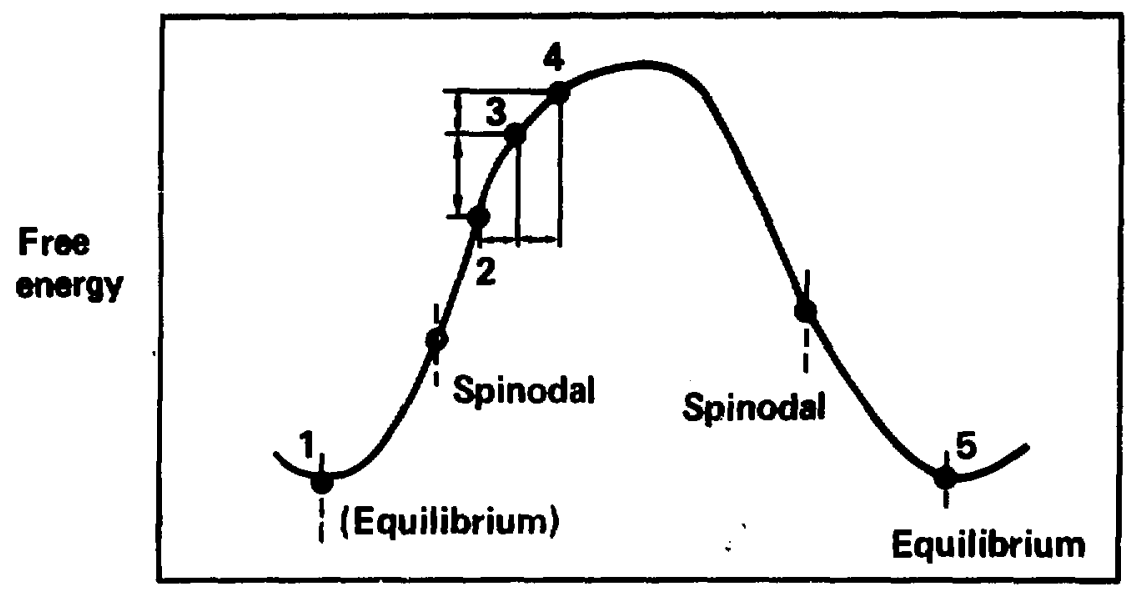

Specific volume or concentration 
Let $\quad \vec{J}=J_{A} \times-J_{B}$

$$
-J=M g\left(\mu_{A}-\mu_{B}\right)
$$

where $M$ is the "dlffusion mobllity" (always positive). The quantity $\left(\mu_{A}-\mu_{B}\right)$ is the free energy change when an "A" atom is reversibly added and " "a" atom is reversibly removed. For a homogeneous system

$$
u_{A}-\mu_{B}=\frac{\partial G}{\partial C_{A}}
$$

Here, $G$ is the Gibbs free energy and the derivative indicates an addition of "A" phase and equal deletion of "B" phase. Conbining the two equations

$$
-J=\left(M \frac{\partial^{2} G}{\partial c_{A}^{2}}\right) \nabla C_{A}
$$

But, by definition

$$
\mathbf{- J}=D_{m} \nabla C_{A}
$$

Hence,

$$
D_{m}=M \frac{\partial^{2} G}{\partial C_{A}^{2}}
$$

Since $M$ is always positive, $D_{n}$ takes its arithmetic sign from the free energy curvature, $\partial^{2} G / 2 c_{A}^{2}$. For spinodal decomposition, there is negative curvature and negative diffusion coefficient. samil concentration (or density) fluctuations tend to grow due to the 
negative diffusion coefficient. In the mucleation and growth region of the P-V diagrem, there is positive curvature and a positive diffusion coefficient. Small density fluctuations tend to die; only large fluctuations (nucle1) which span the free energy peak in Fig. 4.16 can grow.

For a system that is sufficiently inhomogeneous that the inhomogeneity is apparent within the atomic interaction range, the chemical potential is modified.

$$
\mu_{A}-\mu_{P}=\frac{\partial G}{\partial C}-2 K_{S} \nabla^{2} C_{A}
$$

The additional term represents the inhomogeneity effect on the chemical potential wich is a function of the concentration's curvature. The constant $K_{s}$ is positive and a function of the interaction energy and the Interaction distance. When Eq. 4.33 is substituted into Eq. 4.28,

$$
J=M \frac{\partial^{2} G}{\partial C^{2}} \nabla C-2 M k_{s} \nabla^{3} C
$$

By taking the divergence, the diffusion equation for spinodal decomposition is produced,

$$
\frac{\partial C}{\partial t}=\frac{\partial^{2} G}{\partial C^{2}} \nabla^{2} C-2 M K_{s} \nabla^{4} C
$$

As can be seen by back substitution, the diffusion equation has the solution,

where

$$
c-c_{0}=e^{a t} \cos (\vec{\bullet} \vec{r})
$$




$$
\alpha(\vec{\beta})=+H \beta^{2}\left[\frac{\partial^{2} G}{\partial c^{2}}+2 K_{s} \beta^{2}\right]
$$

The second term in the bracket is positive. Outside the spinodal, the curvature is also positive and the amplification factor, $a(\vec{\beta})$ is negative; 1.e, the fluctuations shrink exponentlally with time. Inside the spinodel region, the free energy curvature is negative, leading to positive emplification factors and arowing fluctuations. At large enough frequency $\beta$, the second term in the brackets dominates and the fluctuatlons again die out. Thus, there is a range of fluctuation spatial frequencies that grow in time. One specific spatial frequency grows faster than all others, resulting in a very regular and periodic structure in the decomposing material.

The dominant wavelength is found by maximlzing the bracketed term in Eq. 4.37. Thus

$$
\vec{B}_{\text {dominant }}=-\left(\frac{\partial^{2} G}{\partial c^{2}}\right) / 4 K_{s} .
$$

Cahn estimates dominant wavelengths $\sim 5 \mathrm{~nm}$, and notes that in the early stages both phases are highly connected (rather than isolated bubbles) unless the minority phase is less trien $15 \% .32$

Cann and others have extended the analysis to include crystal enisotropy, coherency stresses, and applied magnetic and elastic flelds. These phenomena add further nonlinear terms to Eq. 4.35, but they only affect the expression for the amplification factor Eq. 4.37; the form of the solution Eq. 4.35 remains unchanged. 30 .

For liquids, the decomposition time is submicrosecond ${ }^{30}$ (less then $10 \mathrm{ps}$ in aef. 33) whlle for sollds nar the molting point it is 
milliseconds. ${ }^{30}$ For cooler solids and glasses, the decomposition tine is long enough to allow experimentation and prectical aplication in materials processing.

For a one component system, flash condensation and homogeneous-nucleation-in-boiling are menifestations of spinodal decomposition. The homogeneous-nucleation-in-boliling process cen be observed by heating a glass container of water in a microwave oven. Because the container is smooth and heat addition is homogaimous, bolling does not originate from cavities at the water-container interface (pool bolling); Instead homogeneous nucleation (spinodal decomposition) begins in the bulk fluid. At this point, the water temperature is much further (perhaps $50^{\circ} \mathrm{C}$ ) above the normal $\mathrm{H}_{2} \mathrm{O}$ bolling point than is the case in pool bolling (which has superheats of less than $\left.5^{\circ} \mathrm{C}\right)^{*}$. Once rapid boiling is underway, the container is removed from the oven, and boiling quickly ceases as the water temperature falls below the spinodal temperature. If a tea bag or other set of nucleation sites is placed in the water before the temperature falls below the pool boiling temperature (about $5^{\circ} \mathrm{C}$ superheat), vigorous phase change will resune, but by nucleation and growth rather than by spinodal decomposition.

* An experiment using a thi mometer with a $225^{\circ} \mathrm{F}$ upper 1 init Indicated no superheat during a rolling boil and at least $13^{\circ} \mathrm{F}$ suparheat after microwave hesting. The equilibrium pressure rise of saturated water between 212 and $225^{\circ} \mathrm{F}$ is $30 \mathrm{kPa}$ ( 4 psi). Since the water was not ejected from the container, the actual pressure rise was neglible, indicating a non-equilibrium state under the two-phase done. 


\subsection{Nucleation and Grouth}

Mucleation and growth are the processes by wich almost all phase changes not involving shock or detonation occur. The references in Section 4.4 bxiefly discuss these processes in contrast to spinodal decomposition. Two additional references containing excellent descriptions of nucleation and growth are Chapter 19 by Gerum ot. al. in that Irensfer in Boiling ${ }^{34}$ and Fronkel's 1946 book Kinetic Thepry of Lioulide (especially Chapters 6 and 7 ). 35

A single phase material that is placed in a density-pressure condition in the metastable region under the two-phase done wi.ll eventually separate into two distinct phases by the processes of nucleation and growth. Nucleation refors to the formation of a minority species nucleus of the critical size. This size can be found from a free body dlagram of a spherlcal vapor bubble (Fig. 4.17). The expansion force due to the internal and external pressures $\left(\pi r_{c}^{2} P_{v}-\pi r_{c}^{2} P_{l}\right)$ must equai the restraining surface force $\left(r 2 \pi r_{c}\right)$. Equating the two forces determines the critical macleus radius, $\mathbf{r}_{c}$.

$$
r_{c}=\frac{2 Y}{P_{y}-P_{l}}
$$

In the equation, $P_{y}$ is the equilibrium vapor pressure at the vapor's temperature, and $P_{l}$ is the surrounding liquid pressure. The aquilibriun is unstable; vapor clusters smaller than the critlcal size collese, and larger clusters grow. This is because the confining on the ver bubble, $P_{l}+2 \gamma / r$, is smaller than the apilituriue vepor pressure for $I$ : $I_{c}$, and the bubble grows. For mextefeal teflus nuclei, the surface forces are so large that they 
Fig. 4.17. Free body diegrem of a critical mucleus. The internal minus external pressure forces are balenced by the surface forces.

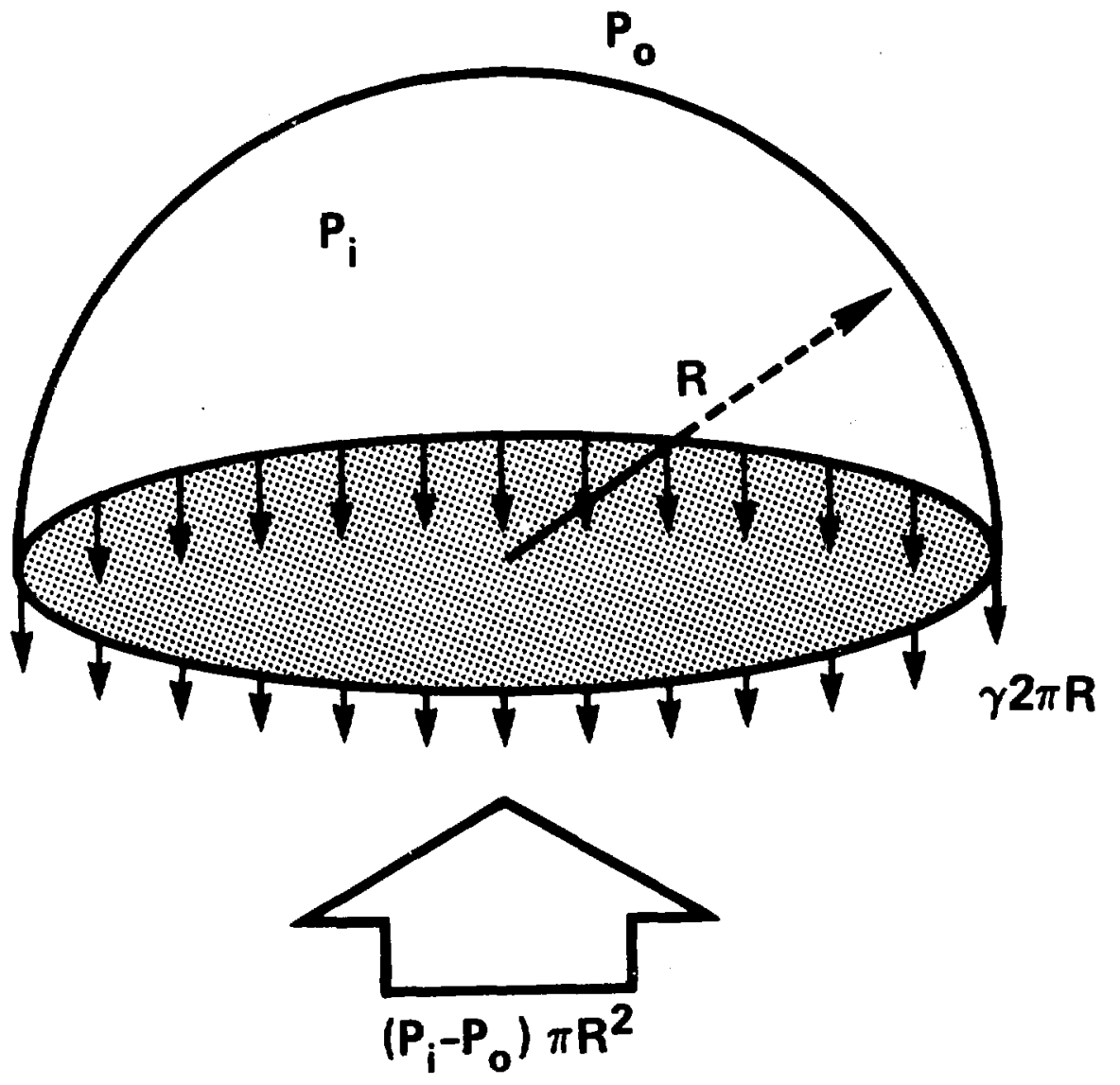


collepse the bubble. In two dimensions, $2 r_{c} P_{v}-2 r_{c} P_{l}=2 \gamma$, or $r_{c}=\gamma /\left(P_{V}-P_{\ell}\right)$.

A second way of thinking about the critical nucleus size is in terms of free energy. The free energy of a drop (or bubble) is approximately the sum of two terms. The first term is the population times the free energy per atom in an infinite system. The second is the surface free energy,

$$
A=N A_{-}+\left(\pi r^{2} \gamma-N T S_{e}\right)
$$

If the brackets, $S_{e}$ is the excess entropy per aton contributed to the systen due to the free surface. Hoover ${ }^{36}$ has shown that for solid crystals with parabolic potentials, this quantity does not vary sharply for systems with more than 100 atoms. To simplify the argument, NTS will be absorbed in the first term. Then

$$
A=\frac{4}{3} \pi r^{3} \rho A_{0}^{*}+\pi r^{2} \gamma
$$

The quentity, $A_{-}^{*}$ is negative and roughly constant $\left(A_{\infty}\right.$ is strictly constant). The surface tension is constant and positive. Enough information has been specified to draw a free energy vs. radius curve (Fig. 4.18). A similar curve is plotted in Ref. 37 for sodium. The curvature at the origin is $2 \pi r$, and the curve peak indicates the critical radius. The free energy at the peak represents an activation mavy for nucleation. Nuclel smaller than the critical radius move to lawer (nore favorable) free energies by shrinking. Nuclei larger then the critical radius lose free onergy by growing; the critical 
radius is an unstable equilibrium point.

The preceding argument intent,ionally avolids providing numbers because, for very small systems, the properties change. As stated earlier, Ref. 36 shows that the excess entropy is a steep function at radil of a few or less. Brodskaya ${ }^{38}$ and Thompson ${ }^{29}$ have both shown that the surface tension falls rapidly from its macroscopic value to zero as the system radius decreases from the 3 to 70 range to zero. This is required by the simple model of Fig. 4.18. In Fig. 4.19, the free energy has been replotted on en energy per atom basis. The high values at small sizes are incorrect. For a single atom, there is no internal energy, and the curve must fall to tha origin as it will if the surface tension falls to zero at the ortigin. Thus, the small radius portion of tine free energy curve is surely distorted, although its shape is probebly correct. Conversely, if the curve is constructed using free energy values measured at radil larger than 60 , using the curvature at $r=0$ to calcu) ${ }^{\circ}$ e the ( $r>60$ ) surface tension is reasonable, with the pi inary erros due to the weak number dependence of axcess entropy at these radil.

According to Gerum ${ }^{34}$, vapor nuclei can form around small (10 $\mathrm{mm}$ or larger) cavities in the container surface (as in pool boiling) or around small ( $<1 \mathrm{~m}$ ) insoluble solid particles. Various types of radiation ( $\alpha, r, n, p$, fission fragments, etc.) have also been suggested as nucleating mechanisms, ${ }^{37}$ elther by depositing the required activation energy in a liquid volume smaller than the critical size, or by cteating a vapor jet that breaks up into discrete regions larger than the critical nucleation size. Kottowski 37 concludes that high energy neutrons in an LMFER would not cause 
Fig. 4.18. Free energy as a function of system size. The maximum defines the critical nucleus size and the nucleation activation energy.

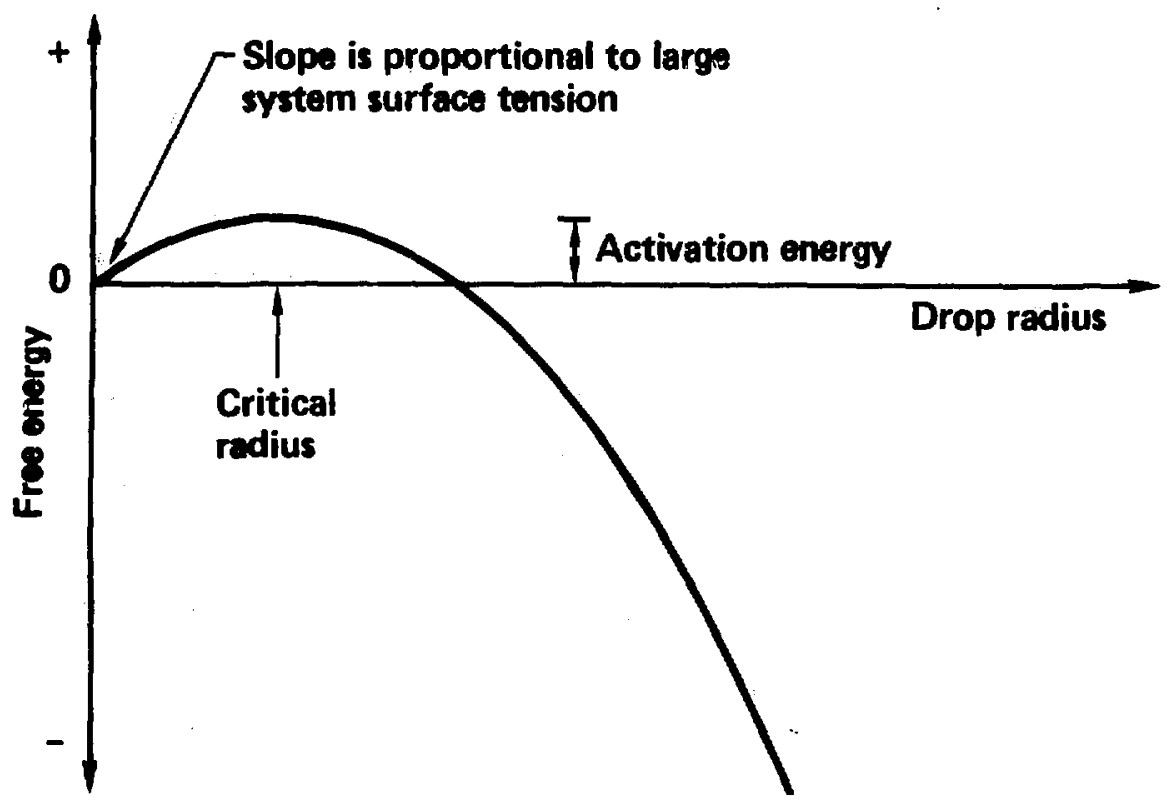


Fig. 4.19. Free energy per atom as a function of systim size for constant and size-dependent surface tension.

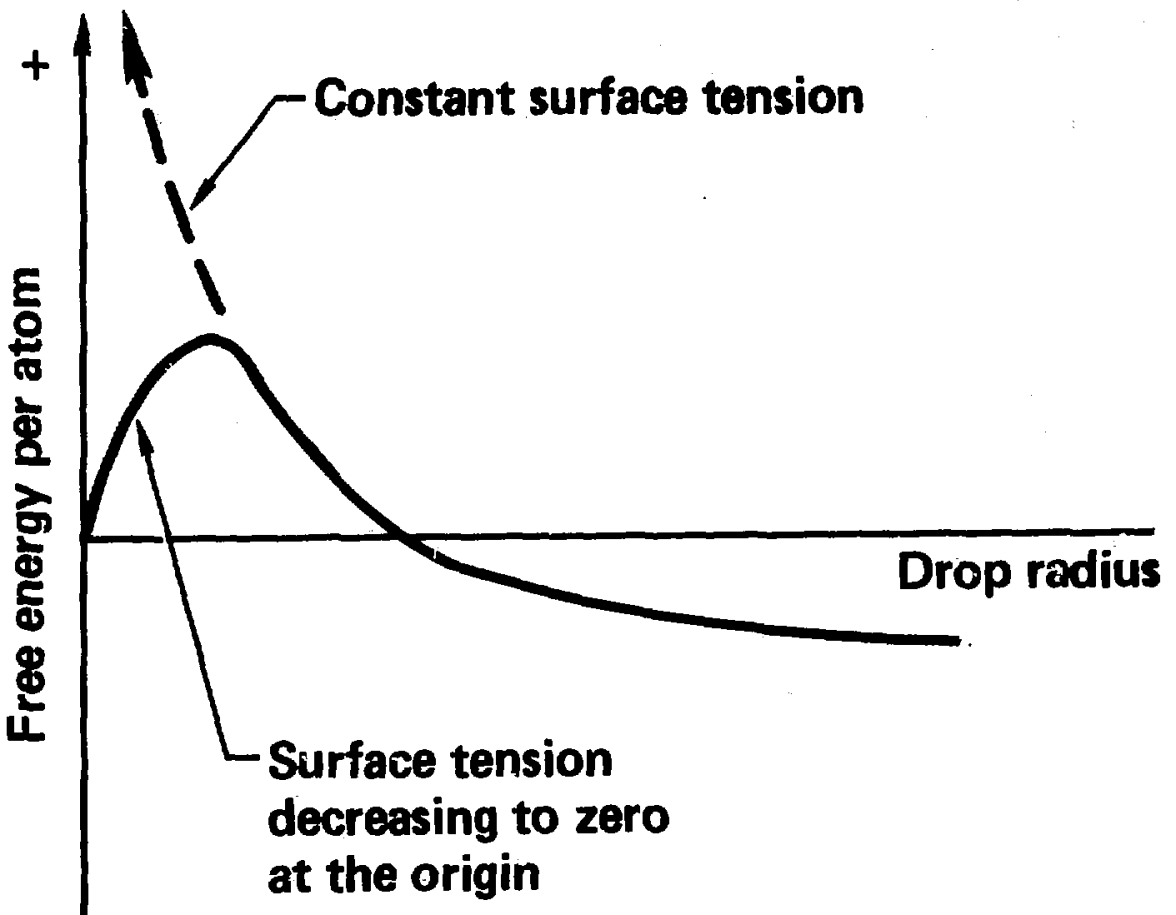


nucleation at $260^{\circ} \mathrm{C}$ superheat in the bulk sodium, but that they could increase the nucleation in wall cavities. Gerum and coworkers ${ }^{34}$ conclude that nucleation from cosmic rays could not account for the moesured superheats in their $\mathrm{H}_{2} \mathrm{O}-\mathrm{SI}$ oll and $\mathrm{CO}_{2}$ experiments.

Frenke ${ }^{35}$ deternined the anount of superheat in a vaporizing liquid as a function of the nucleus size. He began with the clausiusClapayron equation

$$
\frac{d P}{d T}=\frac{H_{V}}{T\left(V_{V}-V_{2}\right)}
$$

The pressure difference between the inside (the vapor pressure) and the outside is $d P=2 \gamma / r_{N}$ where the vapor pressure is at temperature $T$ wich is dT above the saturation temperature. Also, $V_{V} \gg V_{l}$ and $V_{v} \sim k T_{\text {sat }} / P$. Hencel

$$
T-T_{\text {sat }}=\frac{2 Y k T_{\text {sat }}^{2}}{P H_{v} I_{N}}
$$

For large superheat (T-T sat $)$, Eq. 4.42 must first be integrated

$$
\begin{aligned}
& \int \frac{Q P}{P_{l}}=\int \frac{H_{V}}{k T^{2}} d T \\
& \ln \left(\frac{P_{l}+2 Y / r_{N}}{P_{l}}\right)=\frac{H_{Y}}{k}\left(\frac{1}{T_{\text {sat }}}-\frac{1}{T}\right)
\end{aligned}
$$

Thus,

$$
T-T_{\text {sat }}=\frac{k T_{\text {sat }} T}{H_{V}} \ln \left(1+\frac{2 \gamma}{P_{l} I_{N}}\right)
$$

The resulting superheat ( $\left(T-T_{\text {sat }}\right.$ ) is shown in Fig. 4.20 for 
Fig. 4.20. If no large nuclei are present, high supertat is possible before boiling begins.

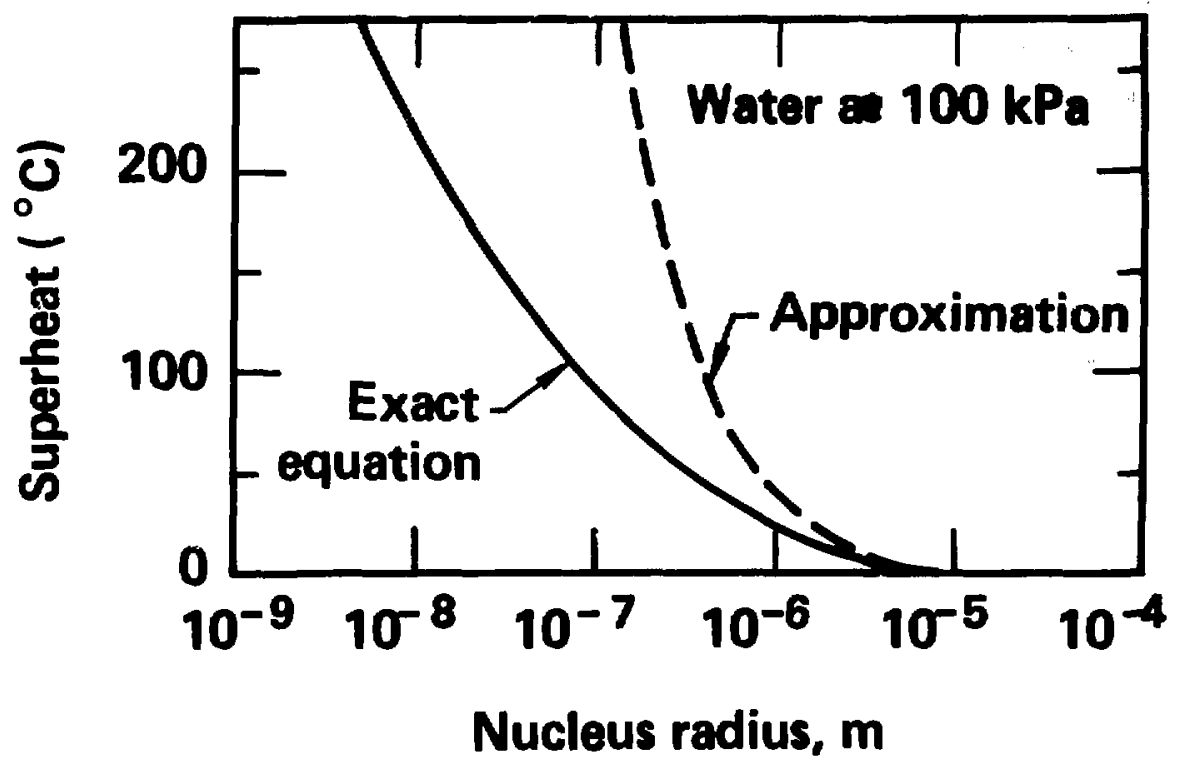


mater at $100 \mathrm{kPa}(1 \mathrm{~atm})$ pressure with the following properties:

$$
\begin{array}{ll}
Y=0.07 \mathrm{~J} / \mathrm{m}^{2} & \text { (surface tension) } \\
H_{V}=7 \times 10^{-20} \mathrm{~J} / \text { molecule } & \text { (heat of veporization) } \\
K=1.36 \times 10^{-23} \mathrm{~J} / \text { molecule-K } & \text { (Boltzmenn's constant) } \\
T_{\text {sat }}=373 \mathrm{~K} & \text { (saturation temperature) }
\end{array}
$$

If no large nuclol ( $r \geq 10 \mathrm{~mm}$ ) oxist, superheats of tens and aven mundreds of degrees are possible. Experiments on water ${ }^{39}$ at $100 \mathrm{kPa}$ have produced superheats of $200-210^{\circ} \mathrm{C}$, and superheats appronching $1000^{\circ} \mathrm{C}$ have been calculated for sodium. 37 The ultimate 1 imit of superheat is reached when the spinodal decomposition regime is entered, 1.e., the system becomes unstuble to small density fluctuations. However, superheat is further linited because the probability of forming smell nuclel $(<\mathrm{nm})$ by density fluctuations is high enough to occur in reasonably short times.

Fluids have been maintained in metastable states by insuring a lack of nucleation sites (for water, at negative pressures up to 27 ma for perlods of severel seconds or more 40,41 , and at negative pressures of 12 MPa for over a week ${ }^{42}$ ). The growth process cannot begin until nuclei larger than the critical size are formed.

Once nucleation has occurred, the vapor bubbles grow to macroscopic size. 39 At early times, -nwth rate is controlled by the inertia of the surrounding liquid, and is described by the Rayleigh equation,

$$
\frac{R_{B}}{d t}=\left[\frac{2}{3} \frac{\left(P_{y}-P_{t}\right)}{P_{l m}}\right]^{1 / 2}
$$

whre $\ln _{\mathrm{m}}$ is the mass density of the liquid. This equation assumes the bubble vepor pressure corresponds to the saturation temperature of 
the bulk liquid. Since the bubble growth velocity, $\alpha_{\mathrm{g}}$ /dt is a constent, the growth rate in this "inertially linited growth region" is independent of tim.

As the growth progresses, the heat, of veporization of the newly created vepor requlres eneroy from the liquid interfece faster then the heat trensfer rate from the bulk liquid. Hence, the interface liquid temperature and the vepor pressure both drop. In this "thermally linited growth reglm", the bubble growth rate is approximated 39 by

$$
\frac{d R_{B}}{d t}=N_{J}\left(\alpha_{t} / t\right)^{1 / 2}
$$

where $a_{t}$ is the thermal diffusivity and

$$
N_{J}=\frac{P_{l m} C_{l}\left(T_{l}^{T}-T_{\text {sat }}\right.}{P_{v} H_{V}}
$$

For the thermally linited growth regine, the pressure Inside the bubble is almost the same as in the bulk liquid, the vapor temperature corresponds to that pressure, and the bubble radius increases with the square root of time. Both regimes are shom in Fig. 4.21.

For $100 \mathrm{kPa}$ mater ${ }^{39}$, the Inertial growth period is < 250 us with velocities $\sim 50 \mathrm{~m} / \mathrm{s}$, and the thermal growth perlod lasts for $\mathrm{ms}$ with velocities of only a few $\mathrm{m} / \mathrm{s}$. These velocities are considerably lower then the expected hydrodynanic olsassembly velocity in the HM.IFE lithlum jets. Thus, bubble growth is too slow to drive the expansion and consequent fragmentation. 
Fig. 4.21. Bubble growth slows with time (from Ref. 39). At early times ( $250 \mu \mathrm{s}$ for $100 \mathrm{kPa}$ water), the surrounding fluid inertia determines the growth rate. At later times, growth is further linited by the rate of heat transfor from the bulk liquid to the interface.

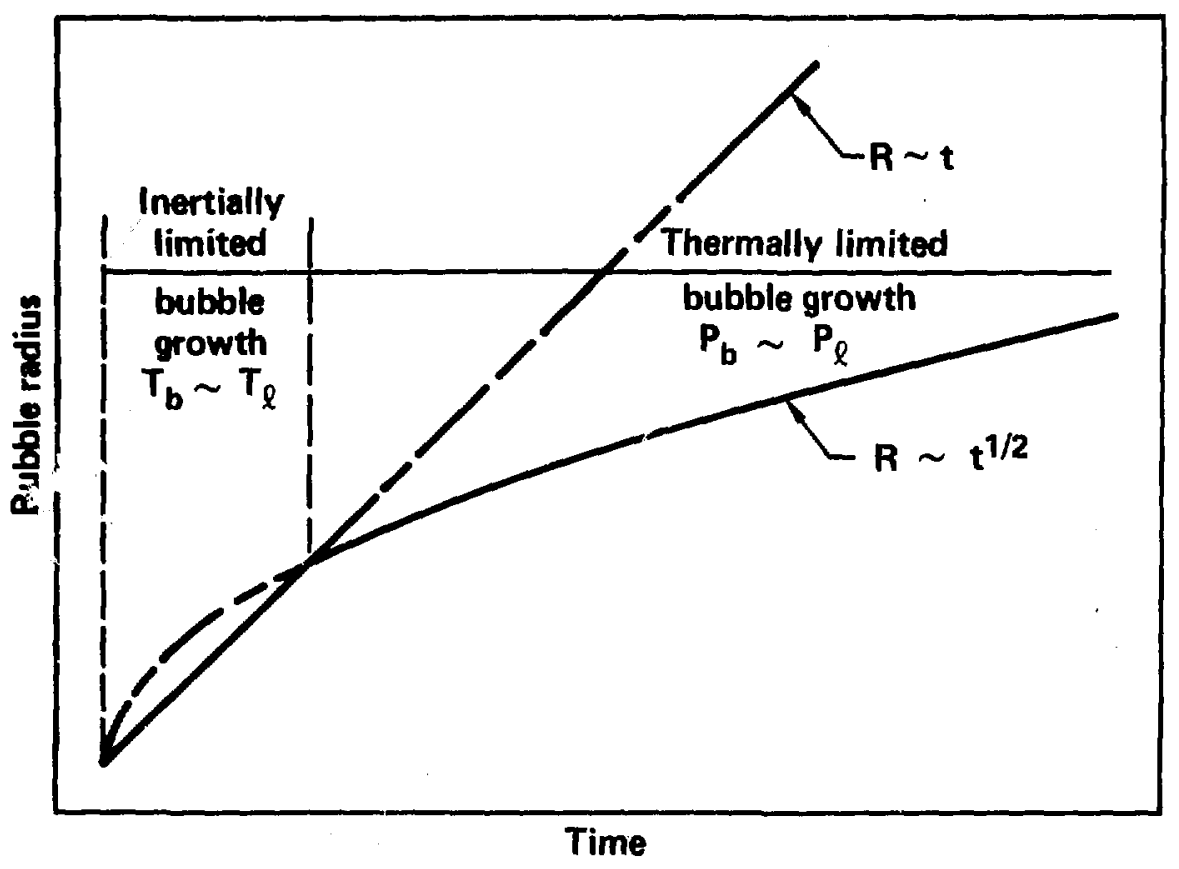


4.6. Evaluation of the Repid Veporizetion Approsech to Liquid Fragmentation

For the $10 \mathrm{~cm}$ radius HMLIFE imer jets, the outmard liquid velocity due to the pressure relief wave is $\sim 100 \mathrm{~m} / \mathrm{s}$ (Table 2.2 ). If the fluid expanded homogeneously until the spinodal line is reached, its specific volume would increase by $\sim 77 \%$. Its radius would increase by $33 \%$, and $180 \mu$ s would elepse during the expansion. When the $\sim 40 \mu$ wave transit tim is added; the tim is still less than the growth time exemples given in Section 4.5 (250 $\mu$ s for the high growth rate initial phase). Hence, nucleation and growth are too slow to drive the expension and consequent fragmentation. If the spinodal line is reached, spinodal decomposition is certainly fast enough, but it has insufficient energy to drive the expansion. Further, hydrodynamic fragmentation would probably occur before the fluid reaches the spinodal line.

It is unlikely that the spinodal line would be reached in a liquid lithium inertial fusion reactor. At the spinode, the liquid tension would be $\sim 1560 \mathrm{MPa}$, much larger than the tensile strength of solid lithium or the tension induced in extremely clean water during carefully controlled experiments. If spinodal decomposition did occur, a characteristic wavelength of coexisting vapor-rich and liquid-rich regions would be -50 , according to simulations ${ }^{43}$ by Abrahem. In an ICF reactor, the entrained gas, target debris, and corrosion products will provide an abundance of potential nucleation sites, allowing a more gradual phase change by the processes of mucleation and growth or by vaporization from the free surfaces of the hydrodynanically fragmented liquid. However, the growth time is 
longer then the liquid's expension time, and vepor growth cennot drive the fragmentation; instead, the expansion leads to fragnentation (as in a solid). The consequent volds equllibrate by fllling with vapor from three potential sources (flow from the vapor originally existing outside the liquid jet or drop, veporlzetion from the new free surfaces, and small vapor bubbles that by move by chance from the fragnent bulk to the now surfeces).

Since rapid phase change does not drive the fragmentation process, at least not for high eneray density reactors like HY.IFE, hydrodynamic models are devised and molecular dynanics simulation are described in the following chapters. 
Chepter 5: Force and Energy Balence Models for Fregantation

Four force and energy bulence models are described in this chapter. The first balances the isochoric heatini pressure by surface tension. The second balences the post-relexation dynemic pressure by surface tension. The third mininizes the sum of the surface energy density and the fraguent non-translational kinetic energy density. (In general, the fregment trenslational kinetic eneroy is much lerger than the non-translational, dilational, kinetic energy.) The fourth model is a modification of the third model in which only the dilational kinetic energy near the fragment surface is considered.

These models differ by balancing different portions of the suddenly added energy against the surface tension. The first model naively requires the surface tension to balance the instantaneously increased pressure. The second model allows pressure rellef and balances only the resulting expansion kinetic energy (dynanic pressure) against the surface tension. The third modal uses only the fraction of the dynamic pressure associated with fragwent dilation; fragment translational energy is properly ignored. Even this model considers only equilibrium situations and round fragnents; thus, experimental verification is required. The fourtin model uses only a portion of the fragment dilational energy.

In this chapter, all four models are described. The first model is revlewed and applied to HYLIFE. Then an Improvement leads to the second model which is also applied to HYLIFE. The previously published derivation of the third model is expanded (In two and three dimensions), and extended to include radially dependent velocity divergence. The third model is also applied to several reector desions, and reformulated 
to allow comparison with the nolecular dynamics results. The fourth nodel is briefly developed as a perturbation of the third model's derivation. Finally, the shortcomings of the models are discussed. 
5.1. Balancing the Isochoric-thating Pressure by Surface Tension An early model (model 1) of fragment size was proposed in 1979. In this model, the pressure increase was balenced gainst the surface tension,

$$
\begin{cases}\Delta P\left(\pi a^{2}\right)=\gamma(2 \pi a) & , 3-D \\ \Delta P(2 a)=\gamma(2) & , 2-D\end{cases}
$$

where $\gamma$ is the surface tension, $a$ is the drop radius, and $\triangle P$ is $p_{m}$ Te as defined by Eq. 2.3. The surface tension, $Y, 1$ is 0.35 and $0.435 \mathrm{~J} / \mathrm{m}^{2}$ for $L 1$ and $L_{17} \mathrm{~Pb}_{83}$, respectively (Table 2.1). Eq. 5.1 reduces to

$$
a=(D-1) Y /\left(p_{m} \Gamma e\right)
$$

where D Indicates 2 or 3 dimensions. For the HYLIFE leading edge, a = $1.8 \mathrm{rm}$, corresponding to a fragment containing 1030 atoms.

The calculation resembles a critical nucleus slze computation except that the internal pressure is determined by the isochoric heating rather than by thermodynanic equilibrium. The model ignores pressure relief in the original liquid drop wich is achieved by generating expansion momentum. Instead, it assums that the original liquid sonetiow fragments such that the added pressure within the liquid can be contained by the surface pressure, $2 \gamma / r$. The calculation also does not predict the original drop size. that would be stable against fragmentation since, if the original drop had radius a, its original internal pressure would be -2y/a before the 1sochoric heating and my/a after heating. 
5.2. Balencing the Post-Relexation Dynamic Pressure by Surface Tension A sacond estimate (nodel 2) of the fragment drop size uses the dynemic pressure $\left(p_{m} v^{2} / 2\right)$ of the expending liquid drop after the 1sochoric-haating pressure has been relleved. Using Eqs. 2.3 and 2.4,

$$
\Delta P=\frac{1}{2} P_{m} v^{2}=\frac{1}{2} P_{m}\left[\frac{P_{m} \Gamma p_{m}}{P_{m} c}\right]^{2}=\frac{1}{2} P_{m}\left(\frac{\Gamma m}{c}\right)^{2} .
$$

Using this pressure in Eq. (5.1).

$$
=\frac{2(0-1) Y}{P_{m}}\left(\frac{c}{f}\right)^{2}
$$

mere $D$ is the number of dimensions. Using the HYLIFE example again a = $90 \mathrm{~nm}$, corresponding to $129 \mathrm{milli}$ on atoms in each fragment. The fragment size is larger than the previous model because the driving pressure is reduced from the isochoric-heating value to the dynemic expansion pressure. The model assumes that the original drop fragments such that each fragnent has an expansion velocity about its center of mass that is identical to the expansion velocily of the orloinal drop. The fragment's surface pressure, 2r/a, resists the dynaic pressure, and the fragwent alternately dilates and contracts until the dymeic dilational pressure is converted to a homogeneous Internal static pressure. The difficulty with this model is that the orlginal expension kinetic energy is partitioned between fragment trwisiation and fragment dilation (in addition to fragment rotation). The nodel lignores the partition, resulting in an cverestimate of the driving pressure and en underestimate of the fregent size. 
$-85-$

5.3. Minimizing the sum of the surface Energy ard the Local dilation Kinetic Energy (The Gredy-Glem Model)

The previous two models ignore the translational and rotation il energies of the fragments. To improve the mods ls, the erection of the kinetic energy (or dynamic pressure) that must belence the surface forces must be estimated. Grady's original effort ${ }^{45}$ was extended by Glom ${ }^{46}$. Their efforts are described end further extended in this four part section. First, the model (model 3) is derived in two and three dimensions. Then, the radially averaged result is extended to include variation with radial position within the original drop. Finally the model is applied to ICF rectors an to the microscopic molecular dynamics simulations. 
5.3.1. Model Derivation in Two and Three Dimensions

Grady uses the fragment size as the independent variable and splits the kinetic energy into the motion of the fragment center of miss ind the dilation about the fragment center of mass (Fig. 5.1). The dilational kinetic energy is added to the surface energy, and the sun is minimized with respect to the fragment size. This energy . minimization procass is similar to the Griffith Theory of Fracture 47 which is famlilar to material sclentists. The surface energy density is

$$
s=\left\{\begin{array}{ll}
\frac{4 \pi x^{2} r}{3} \pi a^{3} & , 3-D \\
\frac{2 \pi a x}{\pi a^{2}} & , 2-D
\end{array}=D r / a\right.
$$

where $D=2$ or 3 for two or three dimensions.

The fragment dilational energy density is

$$
K_{v}= \begin{cases}j^{a}\left(\frac{l}{2} \rho_{m} v^{2}\right) 4 \pi r^{2} d r / \frac{4}{3} \pi a^{3} & , 3-D \\ \int_{0}^{a}\left(\frac{l}{2} \rho_{m} v^{2}\right) 2 \pi r d r / \pi a^{2} & , 2-D\end{cases}
$$

To perform the integration, the dilation velocity must be spacifled as a function of the radius from the fragnent center. This is eccomplished by using the continuity equation in spherical or circuler coordinates with no enoular dependence,

$$
\dot{p}_{m}=\frac{\partial \rho_{m}}{\partial t}=-\nabla \cdot\left(p_{m} \vec{v}\right) \quad, \quad \vec{v} \text { is purely radial }
$$


F1g. 5.1. Geometry used in the Grady-Glem cnergy minimization model. :

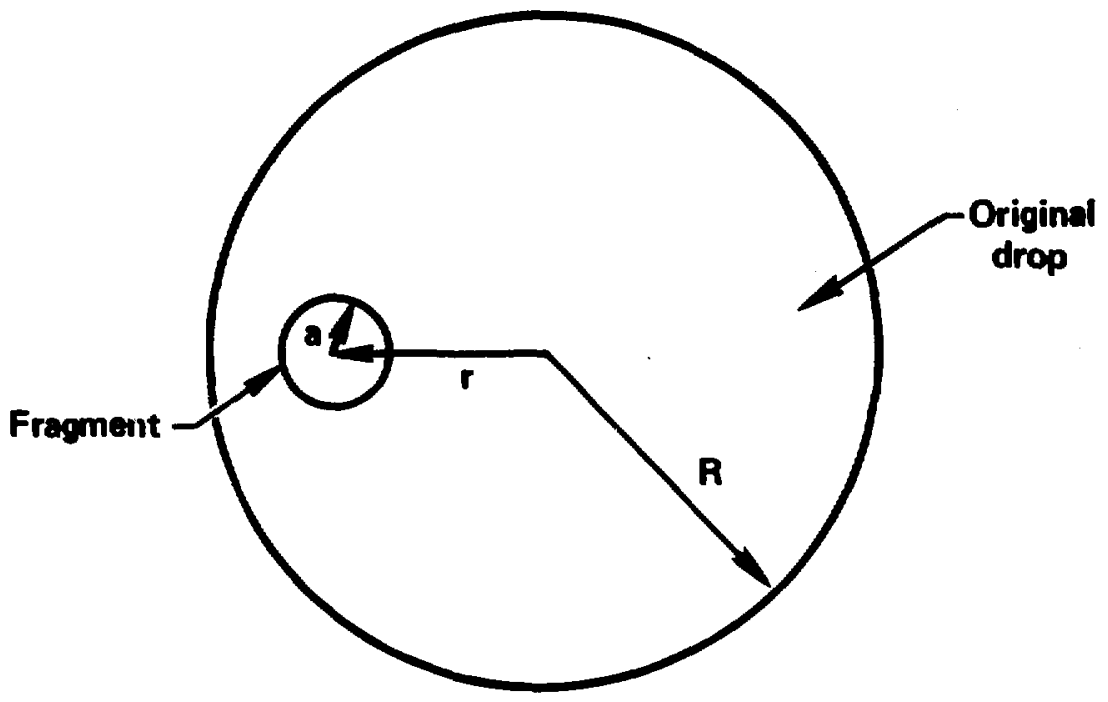


Or

$$
\dot{\rho}_{m}=\left\{\begin{array}{l}
-\frac{1}{r^{2}} \frac{\partial}{\partial r}\left(r^{2} \rho_{m} v\right)=-\frac{\partial}{\partial r}\left(p_{m} v\right)-\frac{2 p_{m} v}{r}, 3-D \\
-\frac{1}{r} \frac{\partial}{\partial r}\left(r \rho_{m} v\right)=-\frac{\partial}{\partial r}\left(\rho_{m} v\right)-\frac{p_{m} v}{r}, 2-D .
\end{array}\right.
$$

For a homogeneousily diverging drop, $\dot{p}_{m}$ is a constent. Then, $\frac{\partial}{\partial r}\left(p_{m} y\right)$ must be a multiple of $p_{m} v / r$. Out $p_{m} v=0$ at $r=0$ by symmatry; hence.

$$
\frac{\partial}{\partial r}\left(\rho_{n} v\right)=\frac{\rho_{n} v}{r}
$$

and

$$
\dot{p}_{m}=-D \frac{P_{m} v}{r}
$$

Using this result, Eq. 5.6 becomes

$$
K_{v}= \begin{cases}\int_{0}^{a} \frac{2}{9} \pi\left(\dot{p}_{m}^{2} / \rho_{m}\right) r^{4} d r / \frac{4}{3} \pi a^{3}, 3-0 \\ \int_{0}^{a} \frac{\pi}{4}\left(\dot{\rho}_{m}^{2} / \rho_{m}\right) r^{3} d r / \pi a^{2} & , 2-0 .\end{cases}
$$

Since it is assumed that $\rho_{m}$ and $\dot{p}_{m}$ don't vary within the Pregment, the integration can be performed,

$$
K_{v}=\left(a \dot{\rho}_{m}\right)^{2} /\left(\mathrm{O}_{2} \mathrm{p}_{m}\right)
$$

where $\mathrm{D}_{2}=20(\mathrm{O}+2)$.

As lllustrated in Fig. 5.2, the sun of the surface energy density and fregent dilational eneroy density is mininized by differentiation,

$$
\frac{2}{2}\left(x_{v}+s\right)=0
$$


F1g. 5.2. Grady's model selects a fregent size that mininizes the sum of the surface plus fregment dilational energy densities.

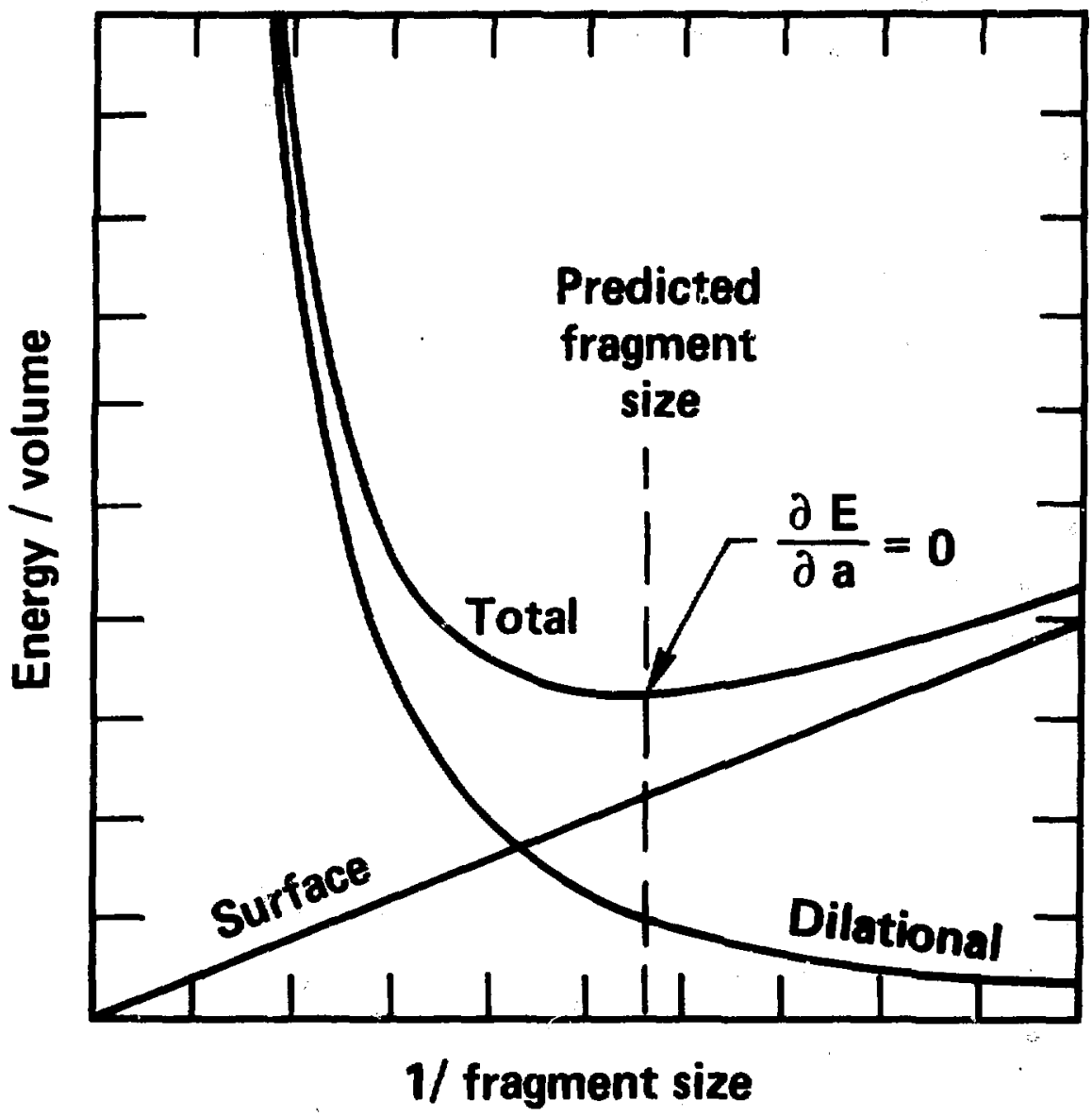


which leads to

$$
0=\frac{2 a \dot{p}_{m}^{2}}{D_{2} P_{m}}-\frac{D_{r}}{a^{2}}
$$

Grady has successfully compared this result to oll shale fragmentation and breakup of explosively loaded pipes (Fig. 5.3). 45

To use Grady's result for ICF reactor desion, the dilation rate, $(\dot{p} / p)$ is requtred. Glem ${ }^{46}$ carrled out a serles of hydrodynamlc calculations of isochorlcally heated (pressurized) spheres. In these calculations, tensile fragmentation was not allowed, and the density varied smoothly. The velocity profile was quite flat for $r / R>0.1$ (Fig. 5.4). Hence, for most of the liquid mass, Eq. 5.9 can be used with $v=v_{s}=[e / c$, the surface (or jump-off) velocity. Then, Eq. 5.13 can be modifled to predict a representative fragment size as a function of initial size (R), energy loading (e), Grüneisen perameter ( $(T)$, sound speed $(c)$, surface tension $(Y)$, and liquid mass density $\left(p_{n}\right)$. The result is

$$
a=\left[\frac{D_{3}}{p_{m}} Y\left(\frac{R c}{r_{e}}\right)^{2}\right]^{1 / 3}
$$

where $D_{3}=D+2$ and $D$ is the number of dimensions.

In most dases, Eq. 5.14 predicts much larger fragnents than models 1 and 2. However, this model includes a new variable, the system size, $R$. If the sphere size is chosen to avoid fragmentation (a $=R$ ), model 3 egrees well with model 2 because then all the kinetic energy is dilationil. For example, with HYIFE loading, the stable (when - R) drop size is $114 \mathrm{~nm}$, within $25 \%$ of the model 2 prediction. The achle drop size prodictions of models 1 and 2 ware 1.8 and $90 \mathrm{~mm}$, 
Fig. 5.3. Comparison of Gredy's model and leboratory experiments (from Raf. 45). Strain rate $15 \dot{p} /(3 p)$ and frecture toughness is $\left.(2 p r c)^{2}\right)^{1 / 2}$.
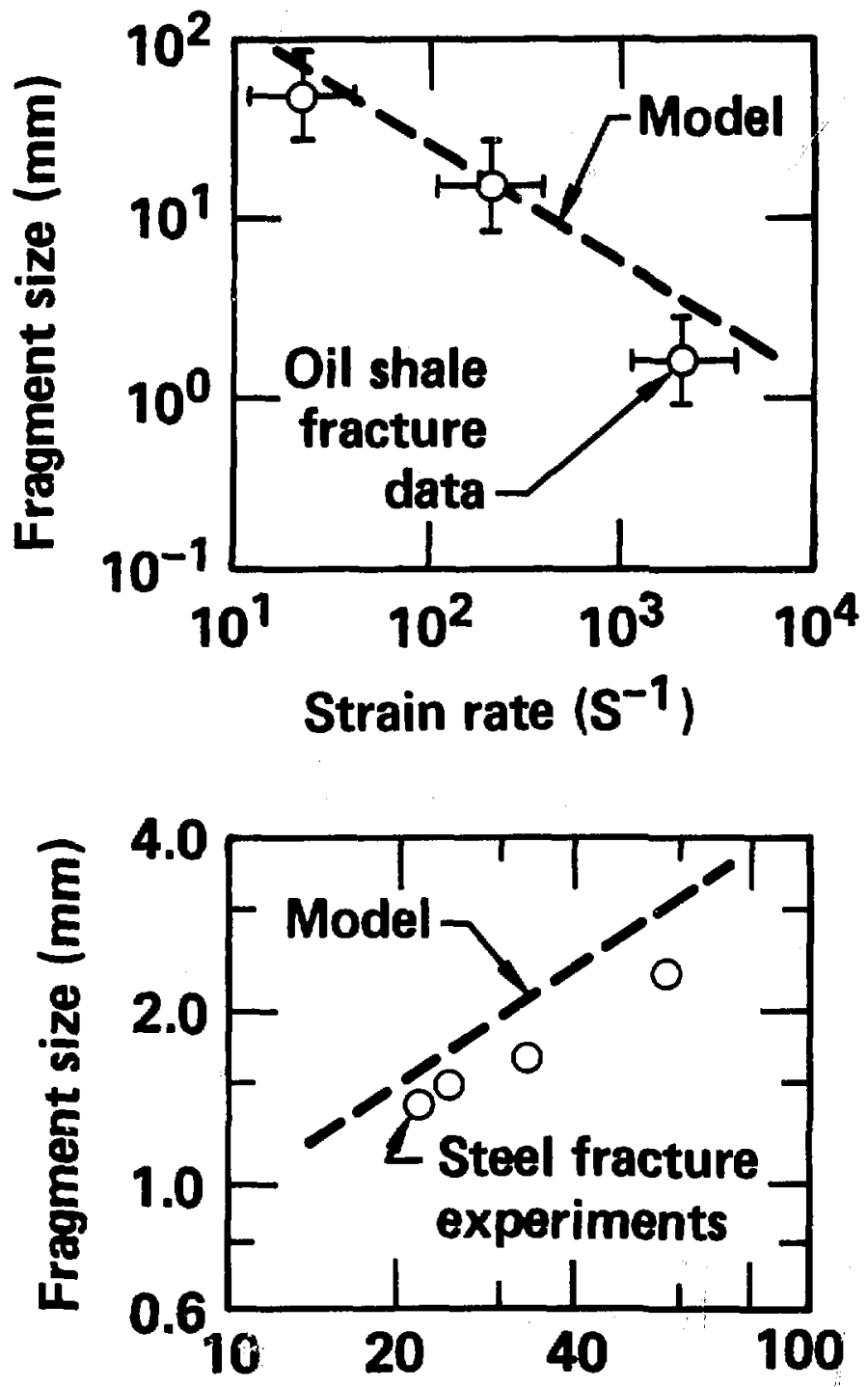

Fracture toughness $-\mathrm{K}_{1 \mathrm{C}}\left(\mathrm{MN} \mathrm{m}^{-3 / 2}\right)$ 
Fig. 5.4. Glem's calculated velocity profile for uniformly heated spheres. The dashed lines are analytic approximations wich are discussed in Section 5.3.2.

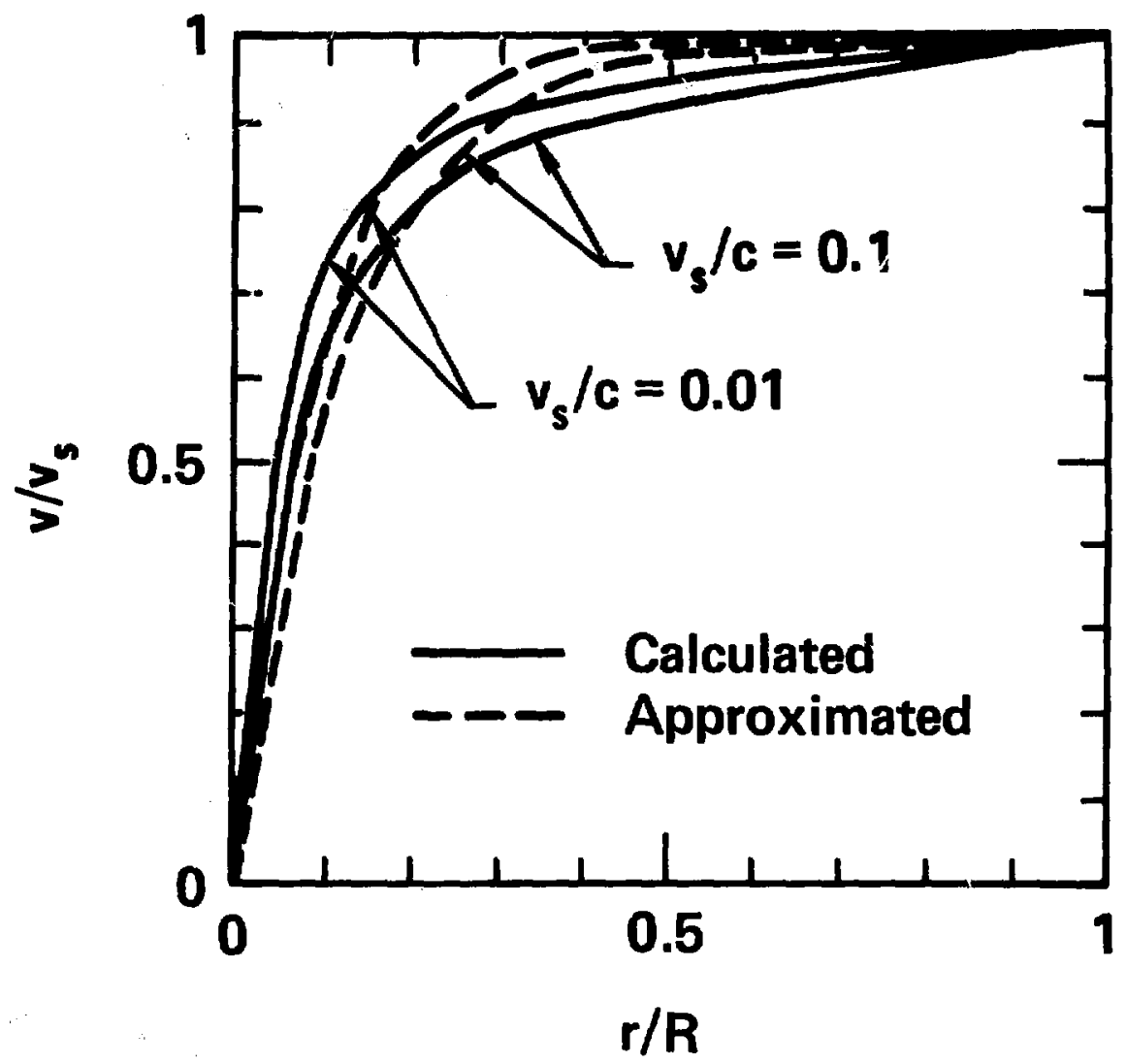


respectively.

The three models balence a decreasing sequence of forces egainst the surface tension. The first model uses thi entire isochoric pressure wile the second model uses the dymemic pressure calculated from the jump-off velocity. Finelly, the third nodel selects only the small portion of the dymanlc pressure confined within the fragment, properly ignoring the portion associated with motion of the fregment as a whole. When the system size and heating level ere such that fragientation is minlmal, models 2 and 3 naturally converge. Similarly, when fragmentation is extensive, nodels 1 and 2 naturally fail.

Another model (model 4) can be derived from model 3 by assuning only the dilational energy contained in the surface region of the fragment must be contained by the surface tension. Equaition 5.6 is integrated only from $(a-\delta)$ to $a$, rather than from 0 to $a$, where $\delta$ is the distance in from the surface beyond wich the dilational energy is ignored. The result of the model is identical in two and three dimensions,

$$
a=\left[\frac{2 y}{p 6}\left(\frac{R f}{\Gamma e}\right)^{2}\right]^{1 / 2}
$$

Model 4 predicts no fragnentation for the HYLIFE example conditions. 
5.3.2. Effect of Considering the Velocity Distribution Rather Than The Average Disassembly Velocity in Model 3

In Section 5.3.1, the fragnentation model (model 3) was derived in two and three dimensions. These derivations used the surface disassembly velocity and constent volocity divergence to predict fraguent size. In this section, the derivation is extended to include the velocity divergence as function of radial position within the orloinal drop rather than a single value. In the derivation, the radial dependence of the velocity divergence is taken from Glenn's hydrodyr:ynic calculations ${ }^{46}$.

Glern approximated ${ }^{46}$ the velocity profile by an exponential expression.

$$
v=v_{s}\left(1-e^{-\mu r / R}\right)
$$

where $v_{s}$ is the surface velocity. As shown in Chapter 2

$$
v_{s}=\Gamma e / c
$$

The approximation (Flg. 5.4) has the correct limiting values at $r=0$ and $I=R$, but is flatter than the calculated velocity profile. The approximation was chosen because it provides a very good fit to the velocity divergence $(-\dot{p} / p)$ as shown in Fig. 5.5. From $r / R=0.1$ to 0.9 , the approximation and the calculation are within $10 \%$ of each other. At $r=0$ and $r=R$, the error is $\sim 20 \%$. The small redfus error is insignificent beceuse the small liquid mass at small redius. The error at the outer redius is evaluated in this section. The constent, $\mu$, is found by/ requiring the velocity divergence 
Fig. 5.5. Glenn's calculated velocity divergence $(-\dot{p} / p)$ and analytic approximaicion.

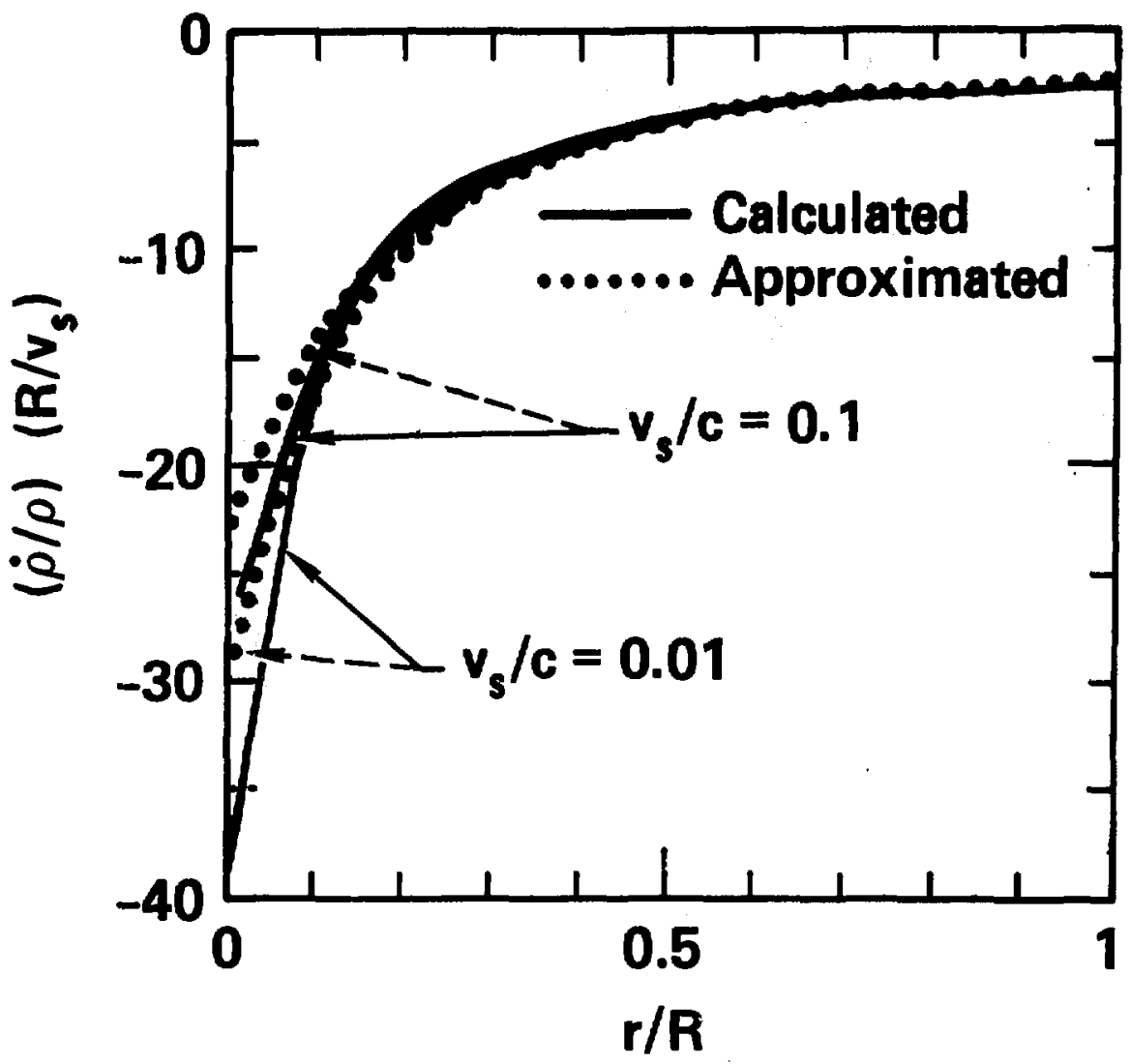


-96-

$(\nabla \cdot \vec{v}=-\dot{\rho} / \rho)$ to watch the hydro-code calculations at $I=0$ and $r=R$.

$$
\begin{aligned}
& -\dot{\rho} / \rho=\nabla \cdot \vec{v}= \begin{cases}\frac{1}{2} \frac{\partial}{\partial r}\left(r^{2} v\right) & , 3-D \\
\frac{1}{r} \frac{\partial}{\partial r}(r v) & , 2-D\end{cases} \\
& = \begin{cases}\frac{v_{s}}{r^{2}} \frac{\partial}{\partial r}\left(r^{2}-r^{2} e^{-\mu r / R}\right) & , 3-D \\
v_{s} \frac{\partial}{\partial r}\left(r-r e^{-\mu r / R}\right) & , 2-D\end{cases} \\
& =\frac{v_{g}}{R}\left[\mu e^{-\mu r / R}+\frac{O_{4} R}{r}\left(1-e^{-\mu r / R}\right)\right]
\end{aligned}
$$

where $D_{4}$ is $D-1$ and $D$ is the number of dimensions.

The two limits are

$$
\left\{\begin{array}{l}
-\dot{R}_{\rho}^{\prime}(r=R)=\frac{v_{S}}{R}\left[O_{4}+\left(\mu-D_{4}\right) e^{-\mu}\right] \\
-\dot{R}_{\rho} I_{(r=0)}=\frac{v_{S}}{R}\left[\mu+D_{4} \mu\right]=\frac{v_{S}}{R}(D \mu)
\end{array}\right.
$$

where lHopital's rule was used to evaluate the second term in the second limit.

The ratio of the two limits is

$$
\left[\left(\left.\dot{e}_{p}\right|_{(r-R)} / \dot{\dot{e}}_{\rho} /(r=0)\right]=\left[D_{4}+\left(\mu-D_{4}\right) e^{-\mu}\right] / D_{\mu} .\right.
$$

For $\gg 1$, the case of interest, the ratio becomes $D_{4} /(D \mu)=$ $(1-1 / D) / \mu$, or $2 /(3 \mu)$ in $3-D$ and $1 /(2 \mu)$ in $2-D$.

$$
w=\left(1-\frac{1}{0}\right)\left[\left.\dot{p}_{p}\right|_{(r=0)} /\left.\dot{e}_{\rho}\right|_{(r-R)}\right] .
$$


For Glenn's 3-D examples, whs 10.4 and 7.9 at mech numbers of 0.01 and 0.1 , respectivaly. The mach numbr, $v_{s} / c$, is much less than one for cases of interest here. The value of $\mu$ can be substituted in Eq. 5.18 to produce a radially-depenient velocity divergence. In Section 5.3.1., this quantity was approxinated as $D_{s} / R$ everymere. For the exponential velocity profile, the surface velocity divergence is $(0-1) v_{s} / R$.

Including the full velocity divergence equation prortuces a correction factor, $v$, in the fragment size equation

$$
a=v\left[\frac{D_{3}}{P_{m}} \gamma\left(\frac{R c}{r e}\right)\right]^{2}
$$

were $\mathrm{D}_{3}=\mathrm{D}+2$. The correction facter, $v$, is a function of the dimensionless position, $r / R$, within the original liquid

$$
v=\left\{\frac{1}{D}\left[\mu e^{-\mu r / R}+\frac{D_{4} R}{r}\left(1-e^{-\mu r / R}\right)\right]\right\}^{-2 / 3}
$$

where $D_{4}=D-1$ and $D$ is the number of dimensions.

The correction factor is plotted in Flg. 5.6 for $\mu=10$. To illustrate the Insensitivity to $\mu$, the curves are also plotted at $\mu=5$ and $\dot{\mu}=20$ (boundaries of the tands). The fragments near the drop center will be up to an order of magnitude smaller then the prediction based on the surface velocity. Based on the 3-0 velocity approximation, the outermost fragments will be up to $\sim 31 \%$ larger than the prediction based on the average velocity divergence. However, the velocity approximation underestimates the velocity divergence at the surface, leading to al overprediction in fregment size. When the actual hydro-code $(-\dot{p} / p)$ value at $I=R$ is 
F10. 5.6. Fragment size correction factor for the Grady-Glenn model. The correction accounts for the fragment's original position within the Liquid drop.

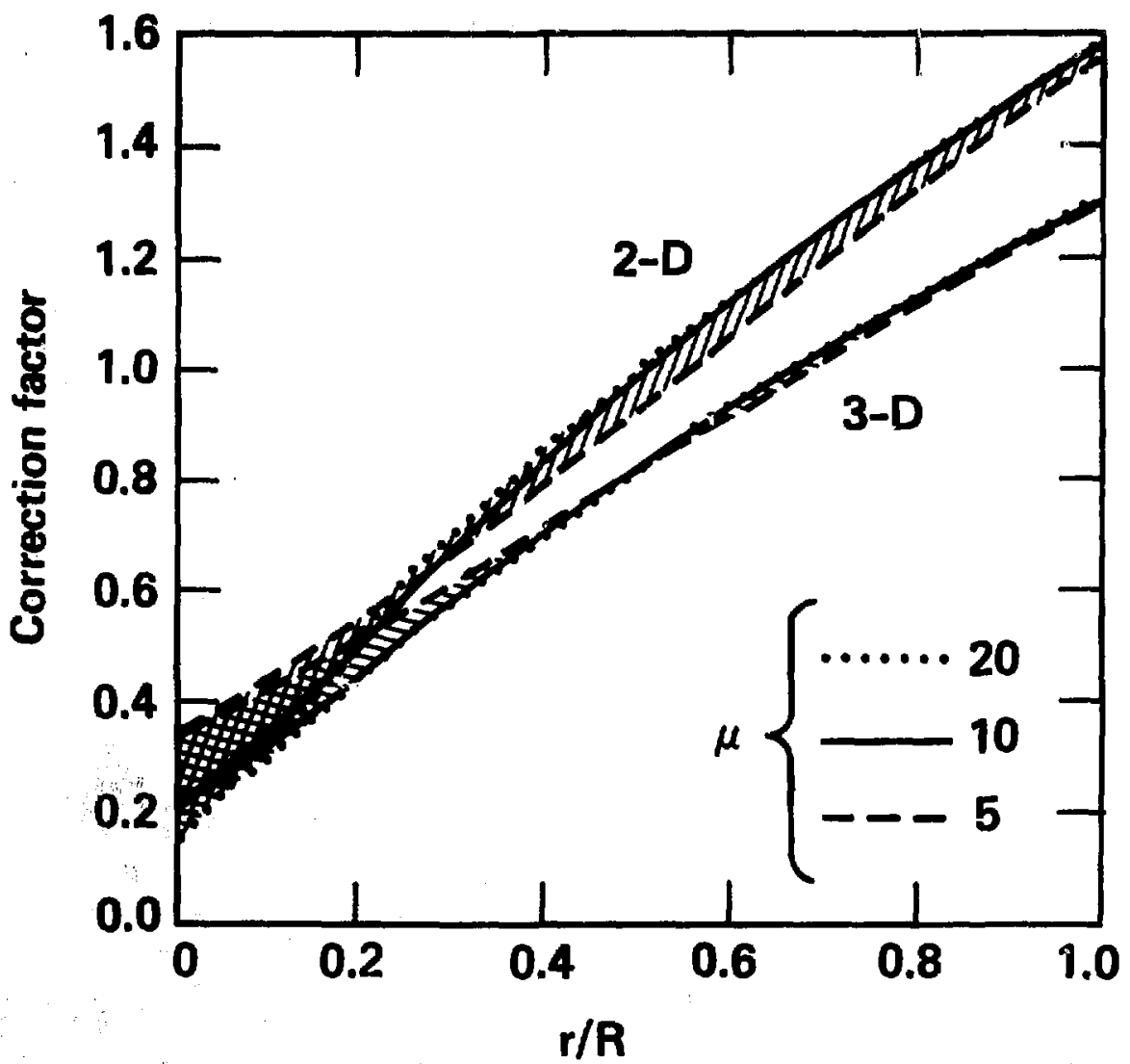


$-99-$

used, the fragment size overprediction from the averaged equation is only 13\%.

Even after this correction, the theory still essive local homogeneity within the fragment (see Eggs. 5.8 and 5.9). Hence, for lite fragment size (a approaching R), the model continues to break down because $\dot{p}$ is not constant in the origins (homogeneous) liquid. 


\subsubsection{Application to Several ICF Reactors}

Toble 5.1 shows the predicted fragment sizes from Eq. 5.14 for the various ICF reactor geomatries described in Teble 2.2. (Simple hydrodynamic calculations of the volocity divergence would also allow redially dependent. fragment size predictions using the mothods of Section 5.3.2.) Table 2.2 also compares the fragment dilational kimatic energy to the fragment translational energy. These values are calculated from the fragment specific kinetic energy (kinetic energy per unit mass),

$$
K_{m}=\frac{1}{2} v^{2}=\frac{1}{2}\left(\frac{\Gamma_{e}}{c}\right)^{2}
$$

and the fragment dilational specific kinetic energy calculated from Eqs. 5.9 and 5.11 with $v=v_{s}$,

$$
\begin{aligned}
& \frac{K_{v}}{p_{m}}=\frac{1}{p_{m}} \frac{\left(a \dot{p}_{m}\right)^{2}}{D_{2} p_{m}} ; \text { and } \dot{p}_{m}=-D p_{m} v_{s} / R \\
& \frac{K_{v}}{p_{m}}=D_{5}\left(\frac{\Gamma e k}{R C}\right)^{2}=D_{6}\left(\frac{Y \Gamma e}{p_{m} R c}\right)^{2 / 3}
\end{aligned}
$$

where $D_{2}=2 D(D+2), D_{5}=D /[2(D+2)]$, and $D_{6}=D(D+2)^{1 / 3} / 2$.

The fragment translational specific kinetic energy is the difference $K_{m}-K_{v} / P_{m} \cdot$ As Toble 5.1 shows, almost all the kinetic energy

is trenslational; coly a small fraction is in dilation of the Pregents.

The Iow energy density reactor concepts are predicted to have 11ttio ar no frementation. For the high energy density HYIFE inner ints, the model (Teble 5.1) predicts fregment diemeters of $\sim 1 \mathrm{x}$ of 
Teble 5.1. Predicted fragmentation in soveral ICF reactars. The fragmentation wes calculated from the formula for unconstrained spheres.

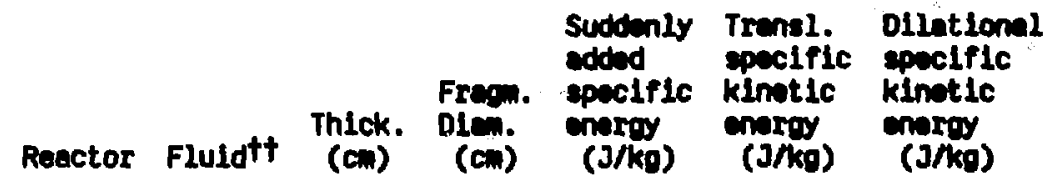

HYI.IFE

Inner jet $\quad$ LI $\quad 20 \quad 0.21 \quad 800,000 \quad 25,800 \quad 1$

HY.IFE

$\begin{array}{lllllll}\text { outer jat Li } & 20 & 1.3 & 50,000 & 61.6 & 0.16\end{array}$

HIBAL.L

(Inner jet, ignoring

LIPb 3

1.8500

0.14

0.013

SiC sock)

Modified

Wetted.

Whilft'

1.14

$2.7 \quad 3500$

0.22

0.081

\begin{tabular}{lcccccc}
\hline EAGLE & LI & 0.05 & $0.14^{\dagger}$ & 3700 & $0^{\dagger}$ & $0.34^{\dagger}$ \\
\hline Pulse*Star & L.LP & 1 & 0.53 & 1000 & 0.58 & 0.042
\end{tabular}

t No breakup predicted by the energy minimization nodel.

tt LIPb is $\mathrm{LI}_{17} \mathrm{~Pb}_{83}$

tft Liquid motion is not in divergling geometry. 
the jet diemeter. The overall HMifE fragmentation will increase the liquid surface area by about two orders of magnitude during the period that the hot gas caused by ablation blows through the jet array (60-300 us after the fusion pulse). After the hot gas blows through the fragmenting array, the fragment transiational energy distribution (higher velocities for the fragments from the inner jets) causes the fragments to coalesce at several ms after the pulse. Then, the coalesced liquid again fragments we to its uniform motion in a diverging geometry. The fragment size distribution during this later period deterwines the condensation rate, at least until homogeneous nucleation begins. The fragnent size distribution during the period the gas transits the array influences the early stages of gas cooling and the structural load imposed when the gas reaches the wall. 


\subsubsection{Application to Sinall Lennard-Jonesicm systems}

The fraginat size predictions of this cheptis have been in tarms of system and fraguent radil. In this section, the models are reformulated into the variables observed in the molecular dyrienics experiments to be discussed in Chepter Seven.

For the moleculer dynmics runs, the fragment data is in torms of population rather then radius.

$$
N_{C}=\rho\left(\frac{D+1}{D}\right)^{D-2} \pi 0^{D}, N=\rho\left(\frac{D+1}{D}\right)^{D-2} \pi R^{D}
$$

The reduced molecular dynamics units allow replacenent of the mass density, $P_{m}$, with the atomic density, p. Finally, the equation of state data (Section 4.2) provides the pressure Increase directly as a function of density and temperature.

$$
P \sim \Delta P=\rho \Gamma e
$$

Thus, the four model predictions become

$$
\begin{aligned}
& N_{c}=\pi\left(\frac{D+1}{D}\right)^{D-2} \rho\left[\frac{(D-1) r}{P}\right]^{D} \\
& N_{c}=\left(\frac{D+1}{D}\right)^{D-2} \rho^{D+1}[2(D-1) \gamma]^{D}\left(\frac{c}{p}\right)^{2 D} \quad \text { (Model 2) } \\
& N_{c}=\left[\pi(D+2)^{D}\left(\frac{D+1}{D}\right)^{D-2} \gamma^{D} \rho^{D+1}\left(\frac{C}{p}\right)^{2 D}\right]^{1 / 3} N^{2 / 3} \\
& N_{c}=\left(\frac{2 \rho Y}{\delta}\right)^{D / 2}\left(\frac{c}{p}\right)^{D} N
\end{aligned}
$$

In two and three dimensions, model 3 is

$$
\begin{aligned}
& N_{c} / N=\left[\frac{16 \pi}{N} r^{2} \rho^{3}\left(\frac{c}{p}\right)^{4}\right]^{1 / 3} \\
& N_{c} / N=\left[\frac{500 \pi}{3 N} r^{3} \rho^{4}\left(\frac{c}{p}\right)^{6}\right]^{1 / 3}
\end{aligned}
$$


The second test of model 3 by the molecular dynamics results is kinetic energy generation. The plenar result (Eq. 5.24) is,

$$
K / N=\frac{l}{2}\left(\frac{\Gamma_{0}}{c}\right)^{2}=\frac{1}{2}\left(\frac{p}{\rho c}\right)^{2}
$$

Glem's $s^{46}$ results show that this equation also approximately applies in spherical geonetry. Howver, the kinetic energy to be measured is the trenslational energy of the fragments; som of the kinetic energy of the jump-off velocity is manifested as dilational energy of the fragments, thus making Eq. 5.34 an upper-bound for the translational energy generation. In two and three dimensions, model 3 (Eqs. 5.24 and 5.25) predicts,

$$
T_{t r}= \begin{cases}\frac{1}{2}\left(\frac{p}{p C}\right)^{2}\left[1-\frac{3}{5}\left(\frac{N}{N}\right)^{2 / 3}\right], 3-D \\ \frac{1}{2}\left(\frac{P}{p C}\right)\left[1-\frac{1}{2}\left(\frac{N}{N}\right)\right] & , 2-D\end{cases}
$$

These equations approach the correct upper bound under extreme fragmentation $\left(N_{c} \rightarrow 0\right)$. However, they do not approach the correct lower bound of zero for no-fragmentation; this is a consequence of the assumption of a homogeneous expansion in model 3.

Becnuse calculation of the surface tension requires use of the molecular dynemics model, numerical predictions are not made until Chepter Seven, after the molecular dynamics model is introduced in Chepter S1x. Also, although the pressure is used directly in Eqs. 5.28 to 5.35 , the specific energy increase, $e$, and the crobeisen . parematar, r, are also evaluated in Chepter Seven to allow comparison of the fictors in the molecular dynamics simulations with the erialogous fectors in the ICF reactor predictions. 


\subsection{Evaluation of the Force and Erergy Balence Models}

The developant of the Grady-Glem model was very exciting because it gave Intuitively reasoneble estiontes of fragment size. However, the model is too simple in that it only considers the lowest final energy state and it only considers sphorical or circular (cylindrical) fragients c The model does not attcmpt to find the path between the initial state and the optimum final state. There could be potential barriers; that 1s, the path between the states might pass through a state of higher eneray. In that case, the opt Lmm state might not be reached during the time of interest.

Isochorically heated drops could begin to break up in two ways. In the first, the growing relief wave could cause cavitation at some depth into the drop, and an onlon skin fragment would result. Since the skin area increases as it expands outward, it will stretch and break, perhaps by some surface instability mechanism. The drop size might then be of order the onion skin thickness. (This mode is expected for films on surfaces, such as the Mwil or the liquid on the outsides of the HIBALL socks.) The second breakup mode would be to fragnent the drop into wedges with radial fracture lines (see Fig. 7.21 for snapshots during breakup). If the wedges are longer than they are wide, they could either collapse into spheres, or they could fragment further due to velocity gradients or surface waves. Breakup by either of these Initial modes could change the local kinetic energy density and, thus, require revised calculations using the Grady-Glem model. Or, they could lead to further non-spherical breakup.

Because the circumferential strain is ideritical to the radial 
$-106-$

strain in a homogeneous expansion, neither the on lon skin nor the wodge modes are expected to dominate (and neither mode does dominate In the molecular dynamics experiments - see Fig. 7.21). 
Chapter 6: Molecular Dynanics Experiment Dovelopment

In Chapter Five, models were developed to predict fregment size and velocity distributions. Grady has tested modal 3 for solid fragnentation, no other tests havo been attcmpted. In this chapter, a molecular dynemics (MD) technique is developed; it will be used to test models 1 to 4 at a microscopic scale in Chapter Saven.

In Section 6.1, previous MD calculations are summrized. In Section 6.2, the equations of motion are formulated in fintte difference form. In Section 6.3, the nodel is applied using a parabolic (harmonic) potential energy function, and the fallure of this potential energy function is explained. In Section 6.4, the Lennard-Jones potential is used to test the mo model. In Section 6.5, a new startup procedure is developed to produce an equilibrium circular system. This procedure begins with a zero-temperature expanded hexagonal crystal. Surface tension implodes and melts the crystal and a moving repulsive wall confines the melted atoms in a circle of the approprlate size. (Without the wall, the system would fragment before the desired initlal conditions are reached.) Then, by repeatedly adjusting the velocities, the desired initial (equilibrium) temperature is established. The resulting configuration and velocities are used to initiate the fragmentation problem with no restraining wall. (Renoval of the wall is necessary to allow expansion and fragmentation.) In Sectlons 6.6 and 6.7 , graphic and tabular output from the molecular dynamics calculations are described. Finally, in Section 6.8, mmory utilization and computational efficiency are fiscussad. 


\subsection{Previous Molecular Dynemles Modeling of Liquids}

Molecular dynamics allows study of flulds at the atomic level.

Once a potential energy function is specified, the equations of motion for all the atoms can be solved and the motion determined as a function of time. Solutior of the equations is straightforward. However, even with the ultra-fast CRAY-1 computer, only a few thousand atoms can be considered. For exemple, in this study, 14491 atoms were followed for a reduced time of $\sim 110$ (17 ps) at an expenditure of - 10.5 hours of CRAY CPU time. Abraham ${ }^{48}$ has followed over 161,000 atoms for $\sim 200$ ps at an expenditure of $\sim 70$ hours of IBM/370-3081 CPU time, yet even this large effort only considered $-10^{-17} \mathrm{~g}$ of material. Thus, macroscopic systems cannot be directly simulated.

Fortunately, study of small systems has shown that macroscopic propertles can be calculated at microscoplc scale. ${ }^{49,50}$ Most molecular dynamics calculations avoid consideration of artificial surfaces around the microscoplc calculation reglon by using periodic boundary conditions. This method works well if the wavelength of the phenamenon of Interest does not exceed the size of the periodic system, the usual case. Reference 51 includes a list of molecular dynamics publications, most of which use perlodic boundary conditions. Hoover ${ }^{49,50}$ has provided several clear examples of the usefulness of molecular dynemics calculations using as few as two atoms with perlodic boundary conditions.

A much smaller number of nolecular dynamics studies have considered finite systems with actual surfaces. Brodskaya and Rusanov ${ }^{30,52}$ studied three-dimensional systems with 32 to 500 atoms for periods of $\sim 50$ ps. They found that the surface tension 
increases from zero to its macroscopic (assmptotic) value as the drop radius increases to $-3.5 \%$. More recently, Thompson, at al. 29 studied three-dimensional systems with 54 to 2048 atoms for much longer periods (70 times nore time steps). They found that although the drop configuration is non-spherical at eny instent, the tim averaged conflguration is spherlcal. Thompson's study also showed that for drop radil greater than 7.50 , there is less then $3 \%$ orror in the Internal pressure due to assumption of constant surface tension and replacenent of the radius of tension by the slightly larger equimolar radius (1.e. replacing $P_{\text {inside }}{ }^{-P_{\text {outside }}}=2 \gamma_{s} / R_{s}$ by $2 \gamma_{-}\left(R_{e}\right)$, and that there is negliglble error for radil $>100$.

For ICF reactor situations, macroscoplc drops are expected to fragnent into smaller macroscopic drops; in this regime, the surface tension is independent of system size. The molecular dynemics simulations should ideally be conducted in a system large enough that the properties are size independent, but computer limitations do not permit such a constraint. In this study, the molecular dynemics calculations used systens with initial radil of $\sim 10 \sigma, 200,40 \sigma$ and $90 \%$. For the smaller fragments from these systems, the predicted surface energy (based on constant $Y$ ) is too large, raising the predicted fragment size above the correct value (Fig. 6.1).

One should also note that the size dependence of surface tension also affects the critical nucleus size. Since the surface forces are smaller than predicted by constant $Y$ for critical muclel with radil less then 7\%, the unstable equilibrium occurs at a higher than expected size, and homogeneous nucleation will be slower then anticlpated (see Fig. 4.19). 
F1g. 6.1. For small fragments, the surface energy is lower than predicted by constent $r$, leading to smaller fragments than expected.

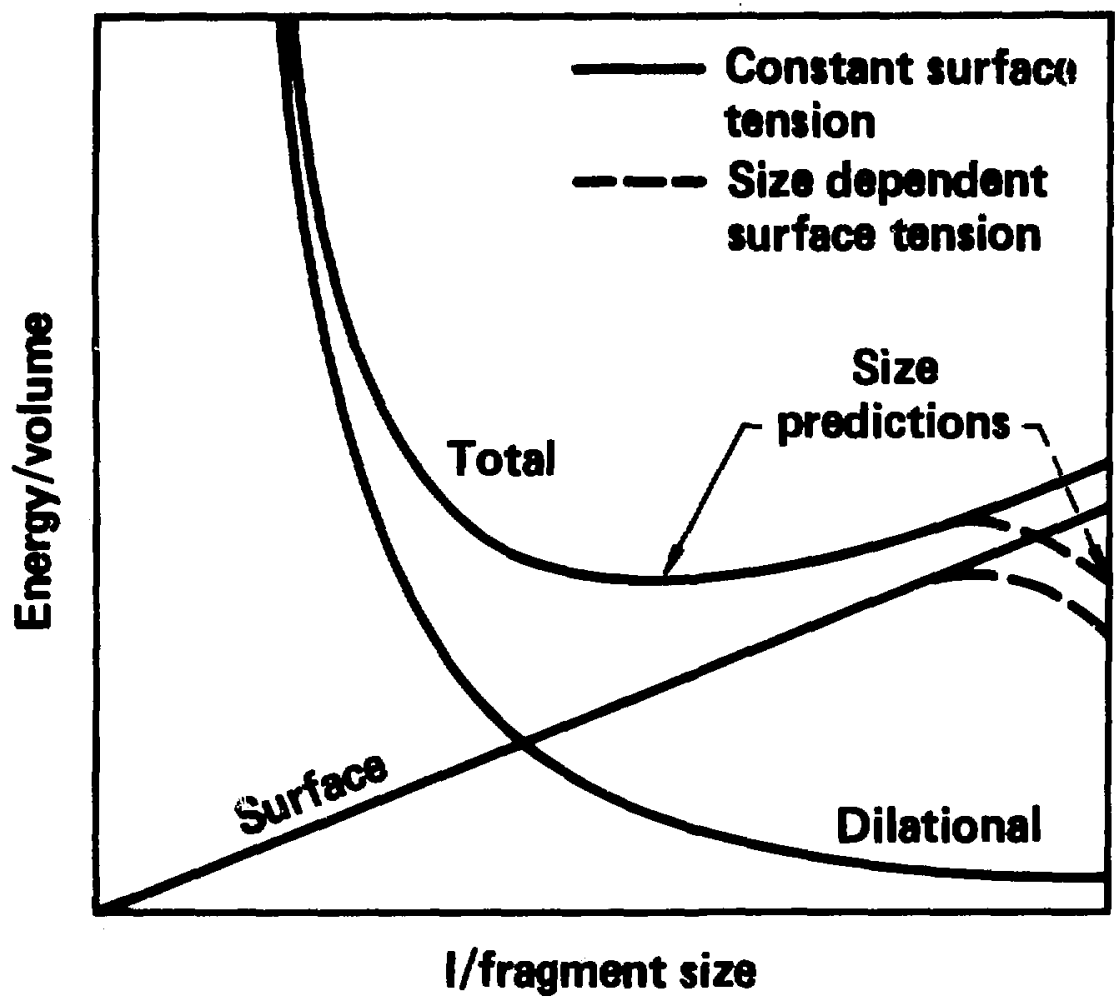




\subsection{Solving the Equations of Motion}

The molecular dynemics nodel was developed in two; rather then three, dimensions for three reasons. First, a largor raglen cen be considered without increasing computation tim. Second, two dimensional motion is easily presented graphically. Finally, for cylindrical jets found in several ICF reactors, expansion in the axial direction is initially negliglble compared to the two-dimensional redial expansion. However, extension of the model to three dimensions is straightforward.

The acceleration for each atom is simply the force per unit mass,

$$
\begin{aligned}
& \frac{\vec{x}_{+}+\vec{x}_{-}-2 \vec{x}_{0}}{(\Delta t)^{2}}=\frac{\vec{F}}{m} \\
& \rightarrow \vec{x}_{+}=2 \vec{x}_{0}-\vec{x}_{-}+\vec{F}(\Delta t)^{2} / m
\end{aligned}
$$

where $\vec{X}_{+}$is the atom location after the next time step, $\vec{X}_{0}$ is the current location, and $\vec{x}_{-}$is the location before the previous time step. The force is found from the potential

$$
\vec{F}=-\frac{\partial \boldsymbol{Q}}{\partial \vec{r}}
$$

The force equation is applieo wo each of the $\left(\begin{array}{c}N \\ 2\end{array}\right)=N(N-1) / 2$ pairs of nodes, where $N$ is the number of atoms. Of course, for nost pairs, the force is zero because a linited range is assumed for the Interatomic potential. For exemple, before fregmentation, only $\sim 10 x$ and $0.5 \%$ of the pairs are non-zero for two-dinensional 127 and 2611 atom systems, respectively. Each pair is checked only once, and $\left(\vec{F}_{1 j}\right)$ is set to $\left(-\vec{F}_{j 1}\right)$. When neighbor listing is employed, only a 
subset of the possible pairs is checked. When adequate motion has occurred, the neighbor list is reconstructed to insure non-zero pairs are not overlooked (see section 6.8).

For startup, the array $\vec{X}_{-}$must be specified.

$$
\vec{x}_{-}=\vec{x}_{0}-\vec{v}_{\Delta t}
$$

Hence, the problem my be started by initially setting the valocitles to zoro and sotting the bonds to non-rest lengths, or vice verse.

If the velocities are zero, it is a zero temperature problien and the node array will initially retaln radial symnetry if the problem starts with radial symmetry (unless either melting or computer round-off errors destroy the symmetry). In this case, the initlel node positions are adjusted from their rest positions

$$
\begin{aligned}
& x_{1}=x_{1}\left(1+\varepsilon_{x x}\right)+y_{1}\left(1+c_{x y}\right) \\
& y_{1}=y_{1}\left(1+\varepsilon_{y y}\right)
\end{aligned}
$$

were $x_{1}$ and $y_{1}$ are the components of $\vec{X}_{1}$ and the strains are functions of the displacenent vector,

$$
\begin{aligned}
& c_{x x}=d u_{x} / d x \\
& c_{y y}=d u_{y} / d y \\
& c_{x y}=\left(d u_{x} / d y\right)+\left(d u_{y} / d x\right)
\end{aligned}
$$

If the temperature is non-zero, the velocity components of all $\mathrm{N}$ particles must be randonly assigned. Flrst, a rendom number, $r_{1}$, betimen $\left(-10(k T / m)^{1 / 2}\right)$ and $\left(+10(k T / m)^{1 / 2}\right)$ is generated. Then a second numer, $I_{2}$, bitiwen 0 and 1 is generated. If $r_{2}\left(-x_{1}^{2} / 2 k T\right)$, the velocity component under consideration is ext te wlus $r_{1}$; if not, two now random nubers are gonerated. This 
procedure results in the Maxwell-Boltzmonn velocity distributien shom in Fig. 6.2.

Once all the initlal velocjtles are selected, they are adjusted so the array has no net Initial minentum.

$$
v_{\text {new }}=v_{\text {old }}-\frac{1}{N} \sum_{1} v_{\text {old }}
$$

Then, the velocitles are adjusted so $\left(1 / 2 \mathrm{mv^{2 }}\right)_{\text {avg }}=1 / 2 \mathrm{kT}$ for each velocity component,

$$
v_{\text {new }}=v_{\text {old }}\left[\left(\frac{k T}{m}\right) / \frac{1}{N}\left[v_{\text {old }}^{2}\right]^{1 / 2}\right.
$$

where the equation is applied separately to the $x$ the velocities. Suming the squares of the new velocities produces $v_{a v g}^{2}=k T / m$ for each somponent.

The time step was set by requiring that energy conservation be maintained. Since the atoms are moved to their new positions riesed on the forces and velocities at thelr current positions, the mc:ion during one time ster must be small enough that the forces don't drastically change. From in energy viewpoint, if a time step reduces an atomic separation into the range where the potential energy function is steeply repulsive, energy is spurlously added to the system. On subsequent steps, the energy will be transferred to these very particles, resulting in rotion of the energized atom past many other atoms in a single tine step.

Equation 6.1 can be written as

$$
\vec{x}_{+}-\vec{x}_{0}=\vec{x}_{0}-\vec{x}_{-}+\frac{\vec{F}(\Delta t)^{2}}{m}
$$


Fig. 6.2. Velocity distribution function used to randonly generate the Initial velocities.

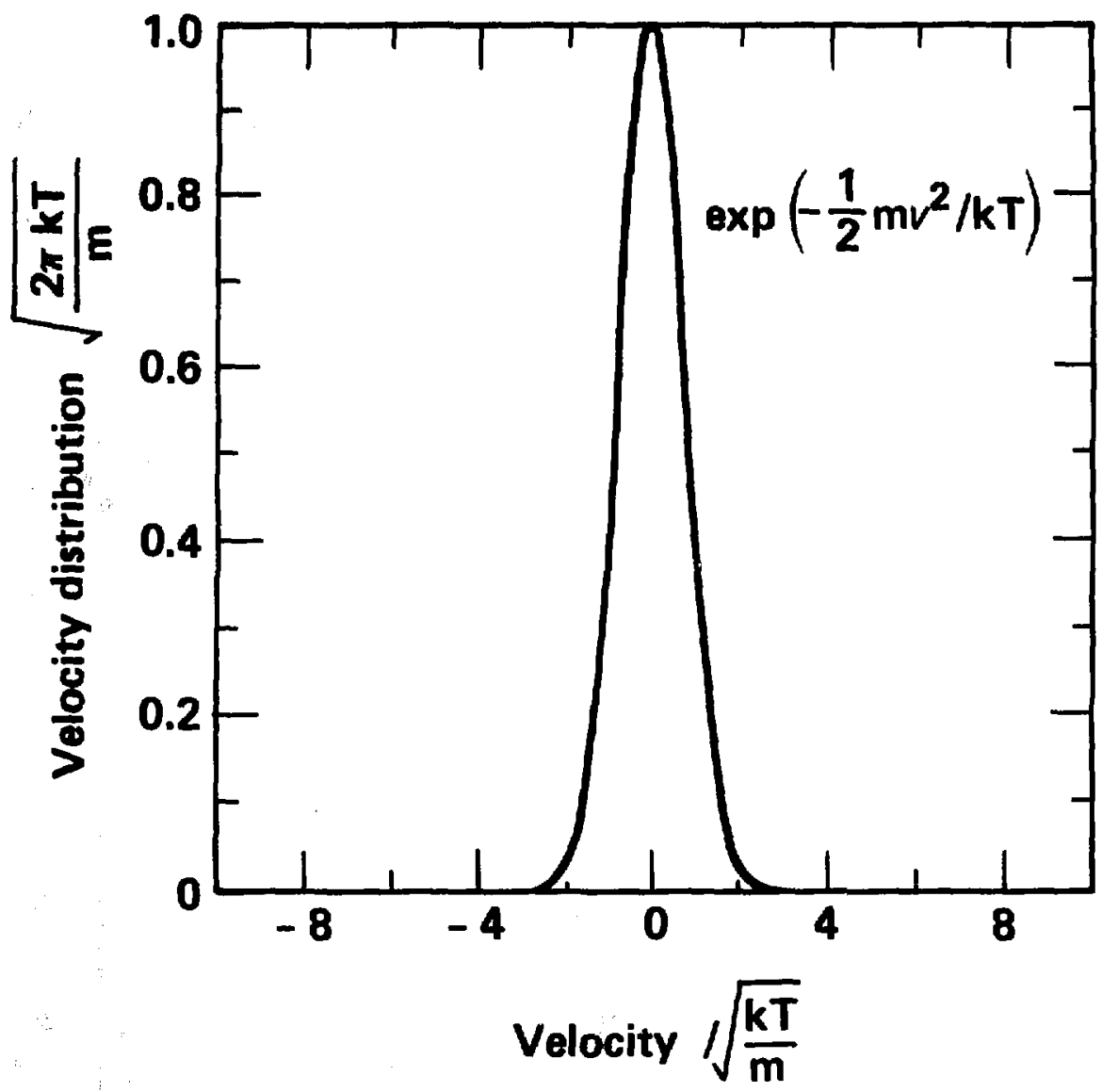


The quantity $\vec{x}_{0}-\vec{x}_{-}$is $\vec{v}(\Delta t)$. If it is assumed that motion, $\left(\vec{x}_{+}-\vec{x}_{0}\right)$, is limited to $10 \%$ of some spocified interatomic specino, $d$, the equation becomes

$$
\vec{d} / 10=\vec{v}(\Delta t)+\vec{F}(\Delta t)^{2} / m
$$

This quadratic equation has the solution

$$
\Delta t=\left(-\vec{v}+\left[\vec{v}-4\left(\frac{\vec{E}}{m}\right)\left(\frac{-\vec{d}}{10}\right)\right]^{1 / 2}\right), \frac{2 \vec{E}}{m} \text {. }
$$

To utllize the equation, values of $\vec{v}, \vec{k}$ and $\vec{d}$ are required. The velocity, $\vec{v}$, is

$$
\vec{v}=\left(\frac{2 \times T}{m}\right)^{1 / 2}
$$

The distance, $\vec{d}$, is the equilibrium spacing for a perfect crystal at the given density or strain (using Eq. 6.4). There are two choices for the force. If the equillorium crystal spacing is small so that a large repulsive force exists, that force is used. If the equilibrium crystal spacing is large so the force is attractive, the maximum possible attractive force for the potential function is used; 1.e., the force at the interatomic separation for which

$$
-\frac{\partial F}{\partial r}=\frac{\partial^{2} \phi}{\partial r^{2}}=0
$$

In the computer code, the default time step was reduced by another factor of ien. Then a time step reduction factor was input during each run. After some experimentation using the LJ potential, a value of 0.4 was found to consistently conserve energy while smoller values did not. Thus, the normal time step is the product of Eq. 6.10 and $1 /(10 \cdot 0.4)$, 1.e., $25 \%$ of equation 6.10 (0.003 ps is typical). 
6.3. The Unsultability of the Parabolic Potential

A simplified potential energy. function for a lithium bond is shown in Fig. 6.3 The function was constructed by first assuming two parcbolic forms that have equal first derivatives at the connection point. This procedure works well for molecular dynamics calculations of solids.

The low point on the potential curve is at the bond rest length $\left(r_{0}\right)$, and it was set so that the interparticle spacing is consistent with the specific volume at the melt temperature $(453.7 \mathrm{~K})$. As shown in section 4.2, $r_{0}=3.123 \times 10^{-10} \mathrm{~m}$ for lithlum. The point at which the two parabolas in the potential join, $I_{j}$, is set by the specific volume at the isothermal spinodal, $V_{j}$. (In retrospect, the isentroplc spinodel would have been a better cholce.) The spinodal point is the place where $(\partial P / \partial V)_{T}=0$. Since pressure is proportional to force or $(-\partial \phi / \partial r)$, the spinodal point corresponds to the inflection point in the potential curve, 1.e., $I_{j}$. If the lithlum is expanding isentropically from an initial condition reached by adding $800 \mathrm{~J} / \mathrm{g}$ to $500^{\circ} \mathrm{C}$ saturated liquid, the spinodal line is reached at a specific volume of

$$
v_{\mathrm{J}}=0.00325 \mathrm{~m}^{3} / \mathrm{kg}=3.745 \times 10^{-29} \mathrm{~m}^{3} / \mathrm{atom}
$$

as shom in Section 4.3. Or,

$$
r_{j}=4.051 \times 10^{-10} \mathrm{~m}
$$

wiene equation 4.21 was used to specify the hexagonal-close-packed 1mer spacing. Thus, the parabolas joln at a bond length of $130 \%$ of the rest length. 
Fig. 6.3. The lithlum parabolic potential enorey function and its associated force function. Positive forces are mepulsive (compression) and negative forces are attrective (tension).

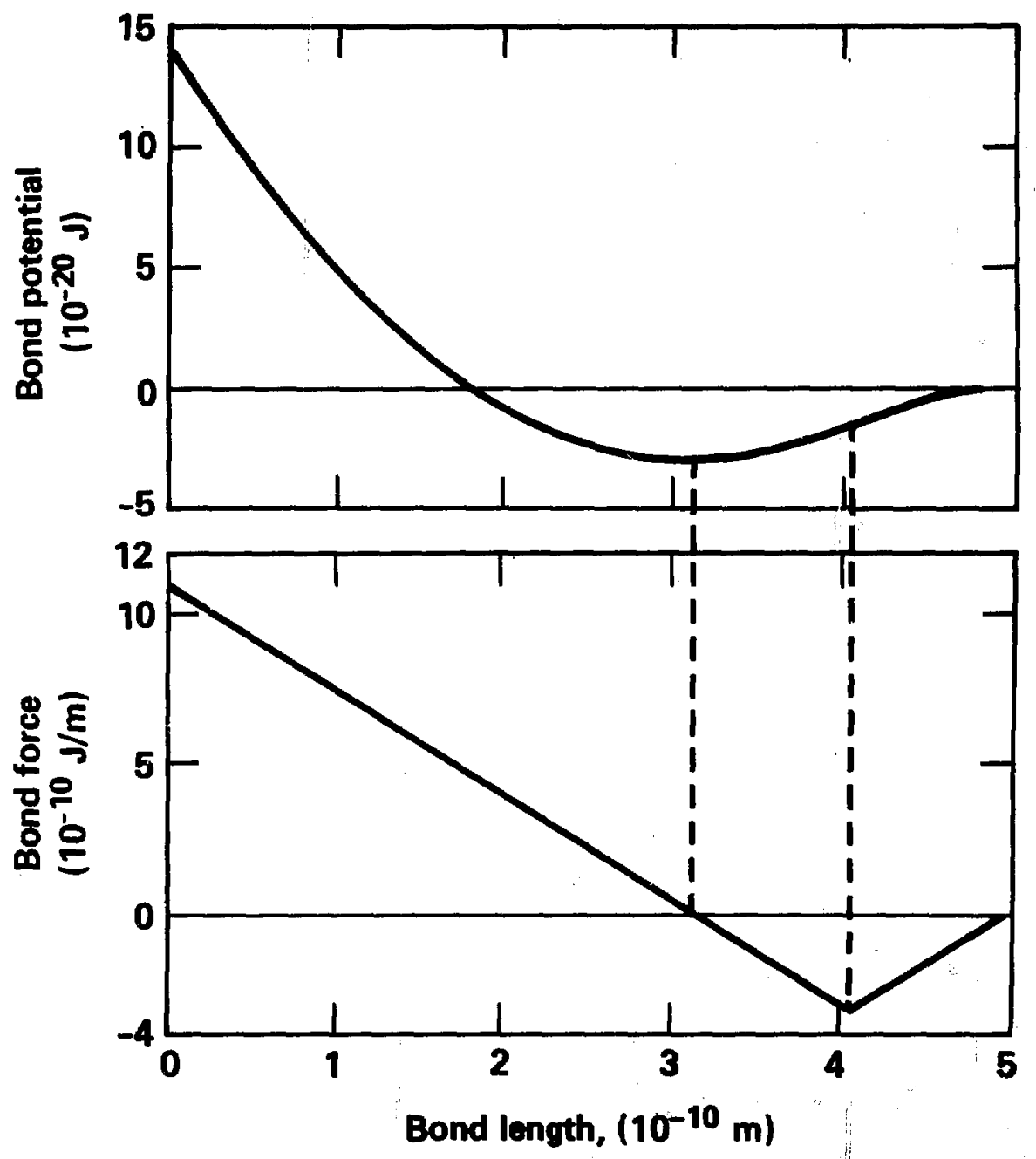


Since the simplified potential function is constructed from two Identical parabolas; only their curvature, $\partial^{2} \phi / \partial r^{2}$, is required to specify the potential function. This quantity can be obtained from the lsentropic bulk modulus

$$
B=-V\left(\frac{\partial P}{\partial V}\right)_{S}
$$

Equation 6.14 can be manlpulated

$$
B=-V\left(\frac{\partial P}{\partial r}\right) \frac{\partial r}{\partial V}=-\left(\frac{N r^{3}}{\sqrt{2}}\right) \frac{\partial P}{\partial r}\left(\frac{\sqrt{2}}{3 N r^{2}}\right)=-\left(\frac{F}{3}\right) \frac{\partial P}{\partial r}
$$

The static pressure is

$$
P=-\frac{\partial E}{\partial V}=-6 N\left(\frac{\partial \alpha}{\partial r}\right) \frac{\partial r}{\partial V}=-\left(\frac{2 \sqrt{2}}{2}\right) \frac{\partial \phi}{\partial r}
$$

where the multiplier, $6 \mathrm{~N}$, Indicates that each of the 12 bonds per atom is shared between two atons. Equations 6.15 and 6.16 can be combined

$$
B=\left(\frac{r}{3}\right) \frac{\partial}{\partial r}\left[\frac{2 \sqrt{2}}{r^{2}} \frac{\partial \phi}{\partial r}\right]=\frac{2 \sqrt{2}}{3 r}\left[\frac{\partial^{2} \phi}{\partial r^{2}}-\frac{2}{r} \frac{\partial \phi}{\partial r}\right]
$$

At the bond rest position (again taken as being at $T_{m}$ ), $r=I_{0}$ and $(2 \phi / \partial r)=0$. Then $\left(\partial^{2}+/ \partial r^{2}\right)=3 r_{0} B_{m} / 2 \sqrt{2}$ for all

I since a parabolic potential function has a constant curvature. The bulk modulus at the melting point is,

$$
B_{i}=-V_{m}\left(\frac{\partial P}{\partial V}\right)_{S}
$$

The factor (aP/oV) $)_{S}$ is the slope on a P-V diagran of the Ientrope passing through the intersection of the saturated liquid amw and the isotherm. When this diagrem was constructed in 


$$
\begin{aligned}
& \left(\frac{\partial P}{\partial V}\right)_{S}=-5.464 \times 10^{12} \mathrm{~Pa} /\left(\mathrm{m}^{3} / \mathrm{kg}\right) \\
& B_{m}=10,550 \mathrm{MPa} \\
& \frac{\partial^{2} \phi}{\partial \mathrm{r}^{2}}=3.495 \frac{\mathrm{J}}{\mathrm{m}^{2}}
\end{aligned}
$$

The equation for the potentlal is

$$
\downarrow= \begin{cases}\frac{1}{2} b\left(r-r_{0}\right)^{2}-c & , r \leq r_{j} \\ -\frac{1}{2} b\left(r-r_{b}\right)^{2} & , r_{j}<r<r_{b} \\ 0 & , r \geq r_{b}\end{cases}
$$

where $r_{b}=r_{j}+\left(r_{j}-r_{0}\right)=4.979 \times 10^{-10} m$ and $b$ is $\partial^{2} \phi / \partial r^{2}$

$=3.495 \mathrm{~J} / \mathrm{m}^{2}$. The curves join at $r=r_{j}=4.051 \times 10^{-10} \mathrm{~m}$. The value of $c$ is found by setting the two equatlons equal at that point,

$$
c=b\left(r_{j}-r_{0}\right)^{2}=3.01 \times 10-20 \mathrm{~J} \quad .
$$

The potential also has a continuous slope where the two parabolas join. A check on the value of $c$ can be made by reallzing that complete vaporization of one atom requires breaking about twelve bonds (in three dimensions) with each bond being shared by two atoms, 1.e., breaking about six bends per atom. Thus, six times the depth of the potentlal well $\left(6 \varepsilon=1.81 \times 10^{-19} \mathrm{~J} / \mathrm{atom}=15.7 \mathrm{~kJ} / \mathrm{g}\right)$ should be and $1 \mathrm{~s}$ comparable to the known cohesive enargy of lithium $(23.03 \mathrm{~kJ} / \mathrm{g})$ less the heat required to convert room temperature lithlum to fust melted Iithlum $(1.03 \mathrm{~kJ} / \mathrm{g})$, 1.e., comparable to $22.0 \mathrm{~kJ} / \mathrm{g}$. Therefore, the value of computed from the bulk modulus and the spinodal density is consistent with the heat of veporization. 
To simplify the initial calculations, reduced units (in wich $r_{0}, m, b$, and Boltzmarin's constent are numerically 1.0 ) were used. In addition, $r_{j}$ was set to $1.15 r_{0}$ (compared to $1.3 r_{0}$ in the actual potential), and $r_{b}$ was set to $1.3 r_{0}$ (compared to $1.6 r_{0}$ in the actual potential). These latter changes reduced the number of wak attractive bonds and dacreased the calculation time.

The resulting simplified parabolic potential is

$$
\downarrow= \begin{cases}\frac{1}{2}(r-1)^{2}-0.0225 & , r \leq 1.15 \\ -\frac{1}{2}(r-1.3)^{2} & , 1.15<r<1.3 \\ 0 & , r \geq 1.3\end{cases}
$$

The well depth of 0.0225 is dictated by requiring the potentlal to be $50 \%$ of the well depth at $r=1.15 r_{0}\left(r_{0}=1\right)$.

The simple parabolic potential was used with an initial condition of $10 \%$ compression of each bond for a 127 atom system. A movie of the atom locations clearly shows the notion of a tensile relief wave from the surface into the center of the atomic array. A second movie of the two-dimensional strain along one axis of the array shows that the tensile wave grows in magnitude as it converges to the center of the array and that the pressure behind the wave is zero. When the wave reaches the center, the bonds linking the central atom with the first surrounding hexagonal row of atoms are broken. A compression wave then moves out to the surface, reflecting as a tensile wave, and the process is repated. Only the first passege of the wave is easily followed since the various reflected waves interfere; however, when the second tensile wave resches the center, the large magnitude of the 
strain and the bracking of bonds are easily recoenlzed.

Since the initial calculation demonstrated the bility to follow waves and break bonds, the lithlum propertles $\left(r_{j}\right.$ and $\left.r_{b}\right)$ wore reincorporated Into the simple parebolic potential for a 127 atom system. A $13.4 \%$ compression initiel condition was imposed, corresponding to $1600 \mathrm{~J} / \mathrm{g}$ if the energy is only in plenar bonds or 800 $\mathrm{J} / \mathrm{g}$ if the energy is in all the bonds (3-D). For this initial condition, each lithlum atom was repulsively connected to the six atons in the first row of nelghbors and attractively connected to the six of the twelve atoms in the second row of noighbors. The initial motion of the outer row of atoms was outward, as before. However, the next row noved Inward since it saw balanced repulsion forces and unbalanced (Inward) attractive forces. The Inward motion compressed a ring and connected other second nelghbor bonds, accelerating the implosion. The outer row turned around, and all the atoms moved to a small central region until over 5000 of the 8001 possible bonds were formed. At this density, many of the bonds were so short that the total of the repulsive forces was large enough to halt the implosion. At late times, there was a tight, non-symmetrical, pulsating core with a number of ejected atoms noving away at hlgh speeds (up to $30 \mathrm{~km} / \mathrm{s}$ ). This configuration is not realistic, as will be discussed shortly.

The Iithium parabolic potential was also used with an initial condition of $445^{\circ} \mathrm{C}$ Instantaneous temperature rise, where the energy was distributed randomly to all bonds in the 2-D mesh. This temperature rise corresponds to $800 \mathrm{~J} / \mathrm{g}$ daposited into 3-D atomic kinetic energy. The velocities moved some of the atoms close enough for second neighbor attractive bonds to form in several regions. The 
attractive bonds pulled more atons into the compressed regions, and the regions themselves converged and coalesced. At late times, the mesh looked qualitatively like the result of the compression initlal condition--a pulsating compressed mass and high velocity ejected atoms.

Since suddenly hented llquids should not implode, the Hooke's law (parabolic) lithium potential must be too simple. One major source of trouble is the smil potential at zero spacing due to the Insufficiently steep parabolic equations. To see if the potential is steble against implosion, the energy per atom of an infinite mesh having the bonds at the rest position was compared to the energy of the same mesh having two or more atoms per site. In the first case, each atom has six bonds at the well depth, and each bond is shared with another atom. Thus, the total 2-D potential per atom is three times the woll depth. In the case with $n$ atoms per site, each atom has one-half share of $6 n$ well depths $\left(\phi_{w}\right)$ and $(n-1) y$-intercepts $\left(\phi_{0}\right)$.

$$
\text { n-atom per site mesh: } \phi=-\frac{6 n}{2} \phi_{w}+\frac{(n-1)}{2} \phi_{0} \text {. }
$$

The potential is unstable if it decreases when the number of atons per sits increases; this condition is satisfied when $\phi_{0}<6 \phi^{*}$ For the simplified potentlal, $\phi_{0}=21.2 \phi_{w}$, and the potential was stcble.

For the Ilthium potential, $\phi_{0}=4.65 \phi_{w}$. Because this value is below the stability limit, implosion to multiple atoms per site is favored. However, the situation is more complex, as can be seen by considoring the four infinite mesh configurations in reble 6.1. The atomic mosh was initially compressed (*2 in 
Table 6.1). The potential with all bonds at their rest lengths ("il in Table 6.1) is energatically favored, but the forces move mich of the mesh in the opposite direction, toward multiple atoms per site (14) wich is energetically favored over both the initial configuration and the rest positions. However, there is en intermedlate configuration with single atoms per site and highly compressed bonds (33) that is favored over all of the other three configurations because there are no bonds with positive potential and many bonds with megative potential. Thus, the mesh implodes, but not completely to multiple atoms per site. The occasional ejection of a few atons of the mash is caused by stress concentrations due to the reflecting tensile waves and by thermal fluctuations.

Because of the failure of the parabolilc potential, the more realistic Lennard-Jones potential is used in the renainder of the molecular dynamics simulations. 
Table 6.1. Potential per aton. The totals are the number of bonds per atom divided by two (for sharing) tines the potential.

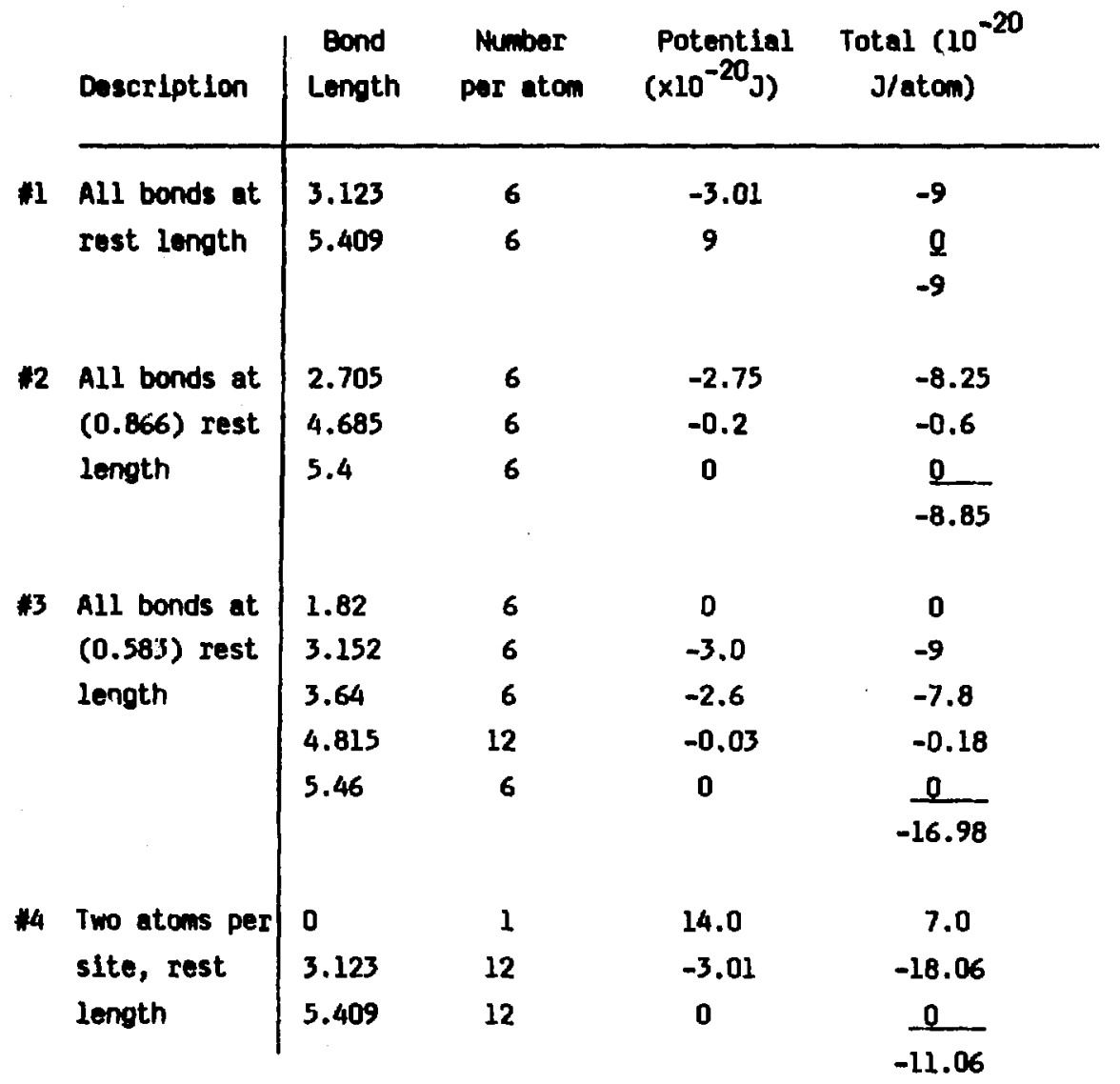




\subsection{The Lennard-Jones (6-12) Potential}

In the previous section, the lithium equation of state was represented by a simple parabolic potential. The potential was unsatisfactory because it was insufficiently repulsive at $\sin l l$ interatonic spacings. In this section, the steeper Lemard-Jones (L.J) potential is addad to the madal.

The Lemard-Jones potentlal and force functions ware shown in Fig. 4.5, and the equations were presented in both SI and raduced units in Section 4.2. That section also showed the $P-p$ and T-P phase diagrans for two-dimensional Lennard-Jonesiun as derived by Barker. 22

The LJ molecular dynamics model (Eqs. 4.25 which use $\sigma=c=k=m=1.0$ ) was tested for a number of initial density and temperature combinations using a 127 atom system. These calculations all began with a perfect solid crystal at the sppropriate density and with a randomly generated velocity distribution that produced the correct kinetic energy corresponding to the temperature. The evolution of the system in tine was then observed. The initial conditions for the test runs are shown in Fig. 6.4.

The first test run (A on Fig. 6.4) was for a cold solid $(p=0.92, T=0.3)$. Floure 6.5 shows the paths followed by the atons during the first 10.2 reduced units of time (1.5 ps). The lattice remalned hexagonal with the atoms moving in small displacements around their equilibrium positions.

The next test run (B) was for a vapor $(p=0.03, T=0.45)$. Figure 6.6 shows a set of four tine exposures of the atom paths. The total elepsed time is 63 reduced units $(9.2$ ps). The long men fres 
F.9. 6.4. Initial conditions for the calculations that tested the molecular dymanics nodel against the published phase diagran.

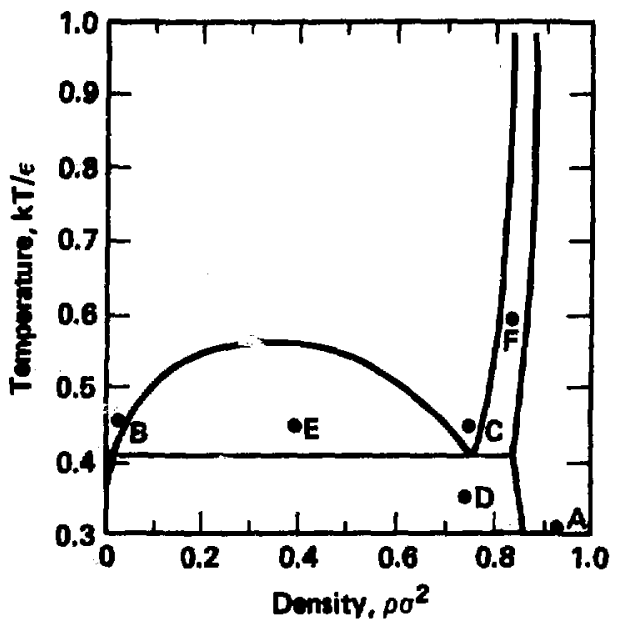


Fig. 6.5. Time evolution of a solid. The atom nover nove far from their equilibrium positions; and the system rateins its shepe." The diamonds mark the final positions of each at at the end of the time exposure. The two parallel lines at the upper left of the fram are scale lirids that should be lonored. The length scalp of 10 reduced units corresponds to $2.8 \mathrm{~mm}$.

\section{Time 0 to 10.23}

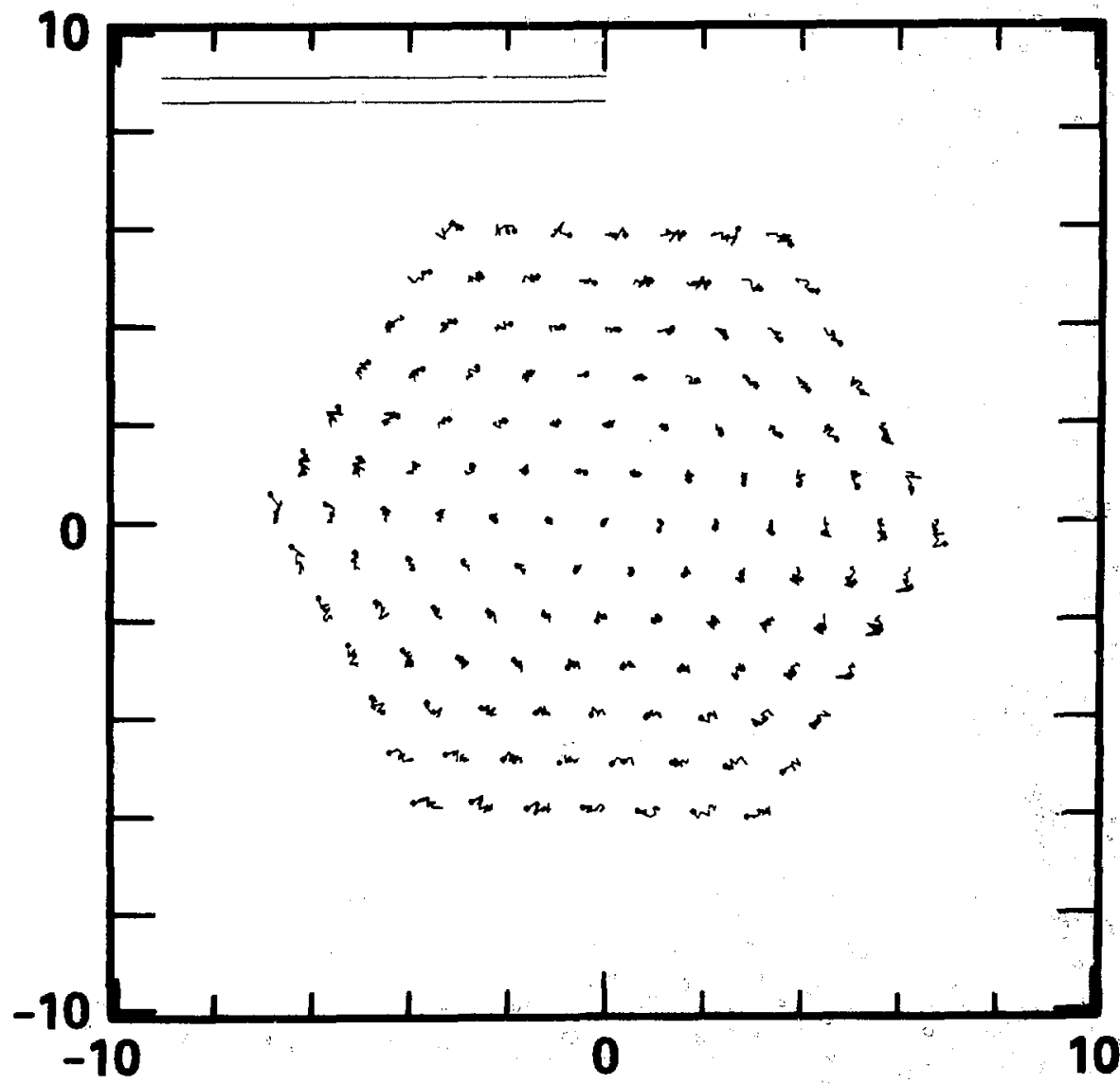


Fig. 6.6. Time evolution of a gas. The four sequential time axposures indicate long mean free paths and an expanding system.

Time 0 to 15.86

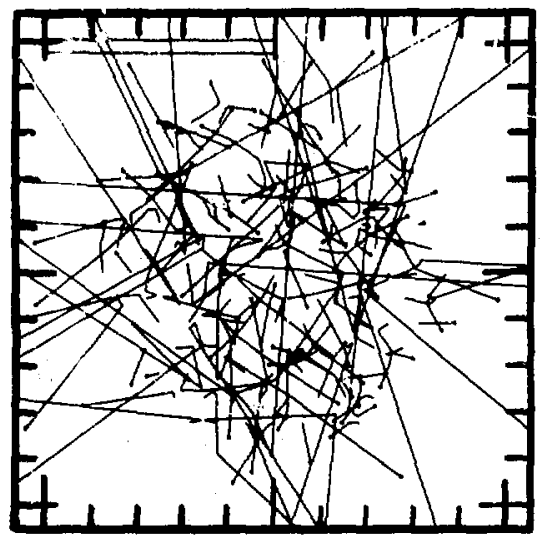

15.86 to 31.56
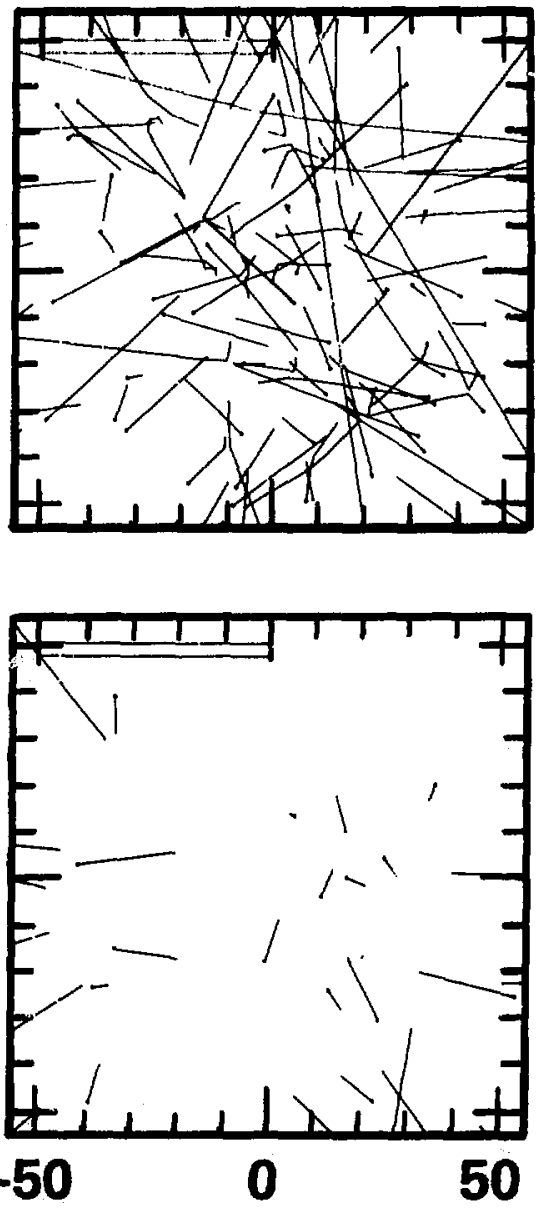

47.26 to 62.96 
paths and expending system are charecterlstic of the veper phase.

Test run $C$ was for a liquid $(0=0.75, T=0.45)$. Flgure 6.7 shows four sequential time exposures of the atcon paths covering 38 reduced tine units (5.5 ps). The vibrations around the equilibritim lattice points are large enjugh to allow atoms to escepe from those equilibrium locations; 1.e., to diffuse through the fluid. A faw atoms near the edge escape the fluid altogether (to establish vapor pressure). Som atoms stay in a crystalline form; this is because the system has relaxed from its initial liquid state to below the triple line.

Test run D was for a solld-vapor mixture $(p=0.75, T=0.35)$. Figure 6.8 shows four sequential time exposures covering 63 reduced time units (9.2 ps). The edge of the initially uniform system is expanding into vapor while the bulk of the system renains as a crystalline solld.

Test run $E$ was for a liquid-vapor mixture $(p=0.4, T=0.45)$. Figure 6.9 shows four sequential time exposures covering 15 reduced time units (2.2 ps). The initlal uniform density region quickly separates Into liquid drops and isolated vapor atoms.

Test run F was for a solid-liquid $m$ ixture $(p=0.85, T=0.6)$. Figure 6.10 shows four sequential time exposures covering 32 reduced time units ( $4.6 \mathrm{ps})$. The core remains solid while the outer atoms move around the outer edge like a liquid film.

The purpose of these test runs was to qualitatively observe the evolution of systems beginning in various regions of the phase diegrem. Test runs A, B, E, and F (solid, vepor, liquid-vapor and solid-liquid) evolved into the expected equilibrium figurations. Test 
Fig. 6.7. Tim ovolution of liquid. The four sequential tise exposures show the diffusion of liquid atoms throughout the system, esteblishment of vepor pressure and relaxation to balow the triple line (since som regions appear to be solid).

Time $=0$ to 9.42
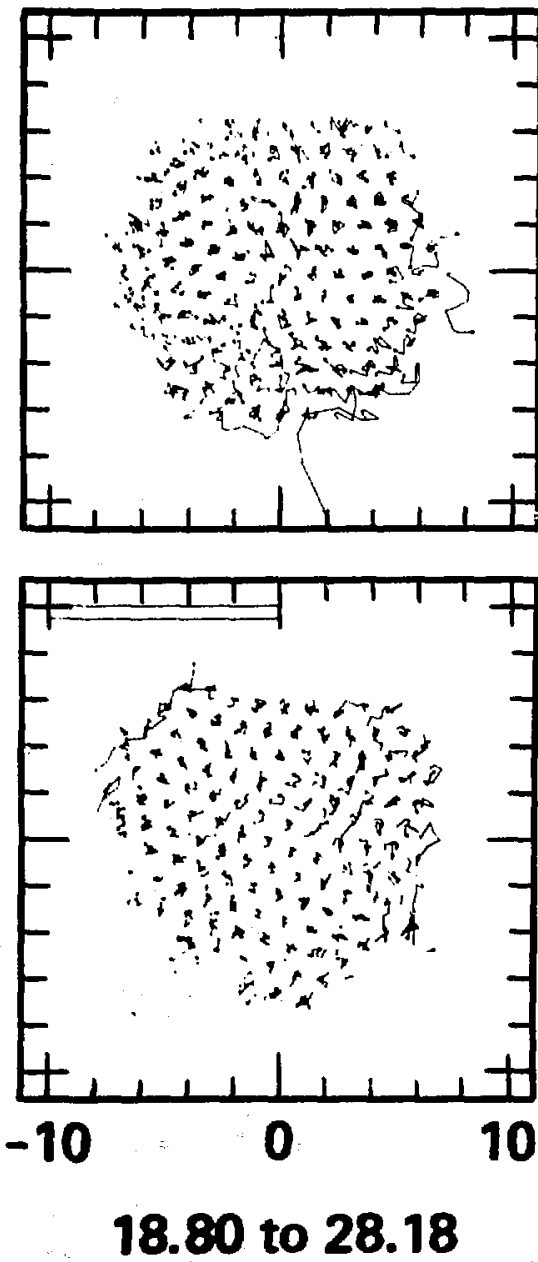

9.42 to 18.80
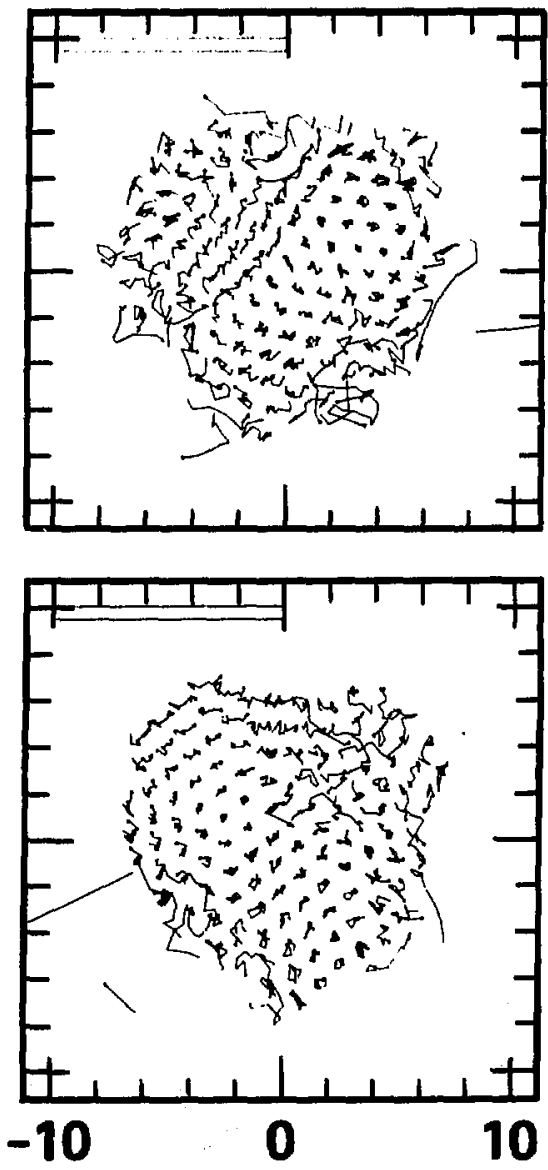

28.18 to 37.56 
Fig. 6.8. Tim evolution of a solld-vepor stete.

\section{Time $=0$ to $15.76 \quad 15.76$ to 31.46}
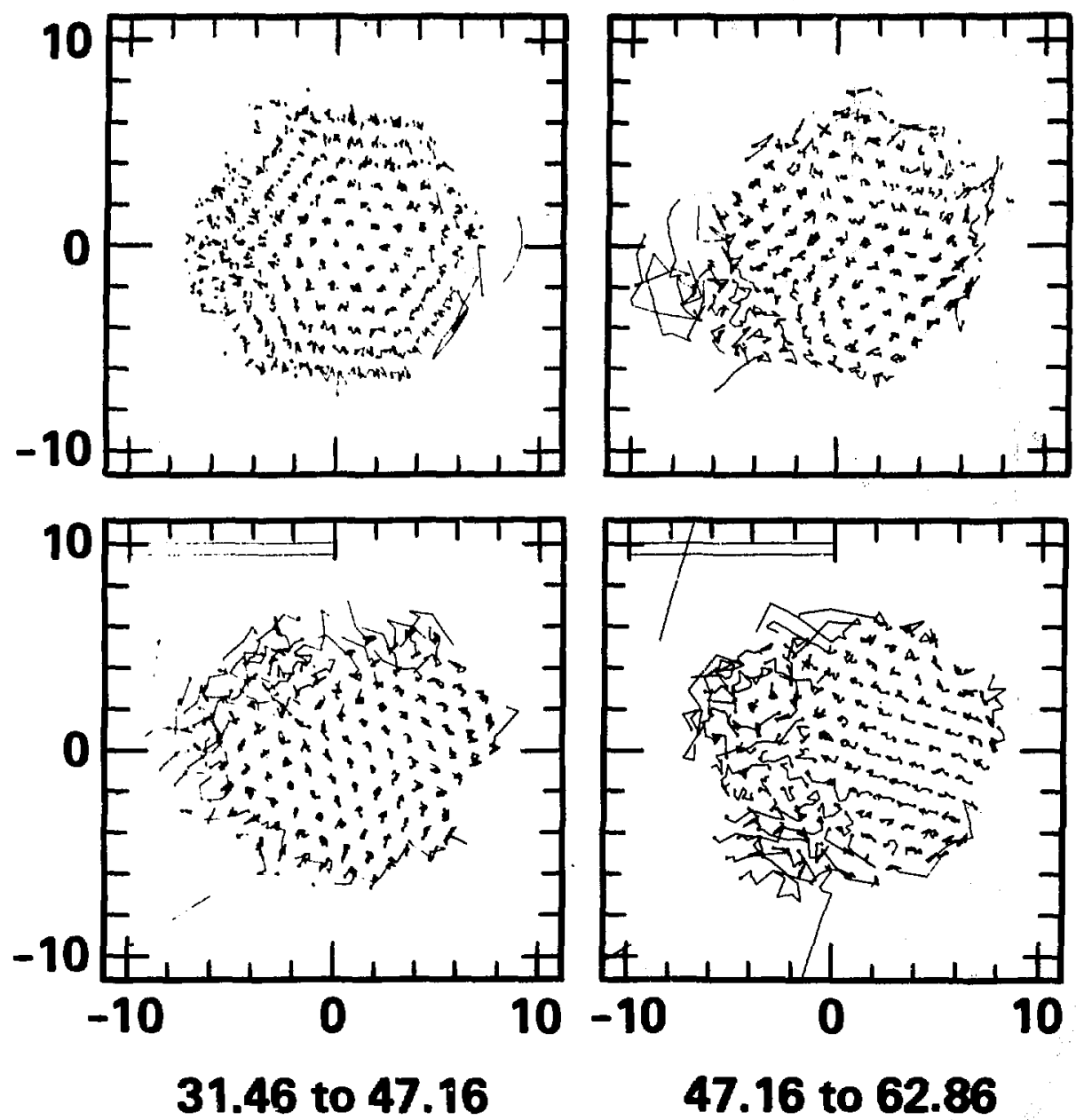
FIg. 6.9. Tim evolution of a liquid-vepor state.

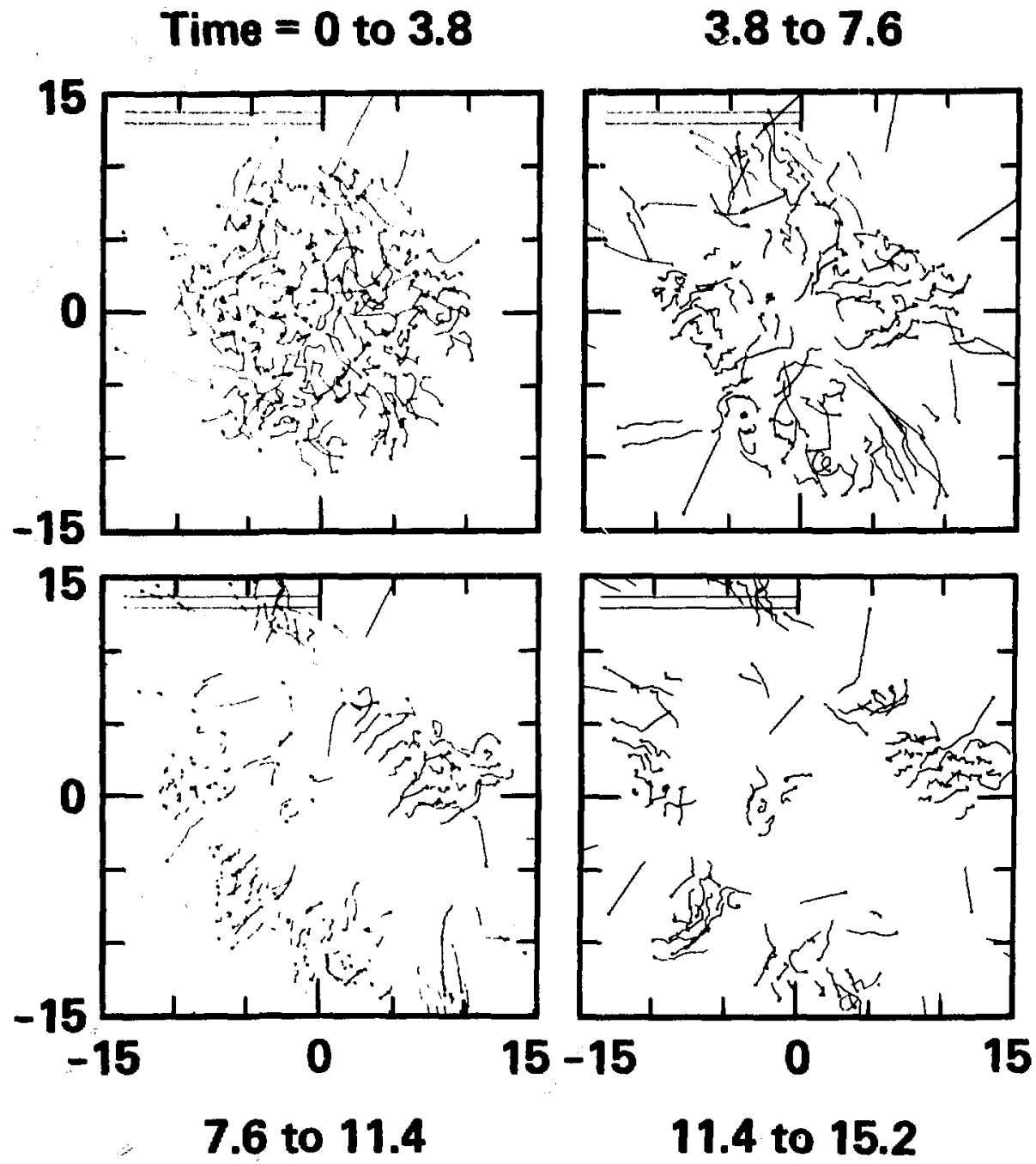


Fig. 6.10. Tim evolution of a solid-1lquid stete.

Time 0 to 7.9
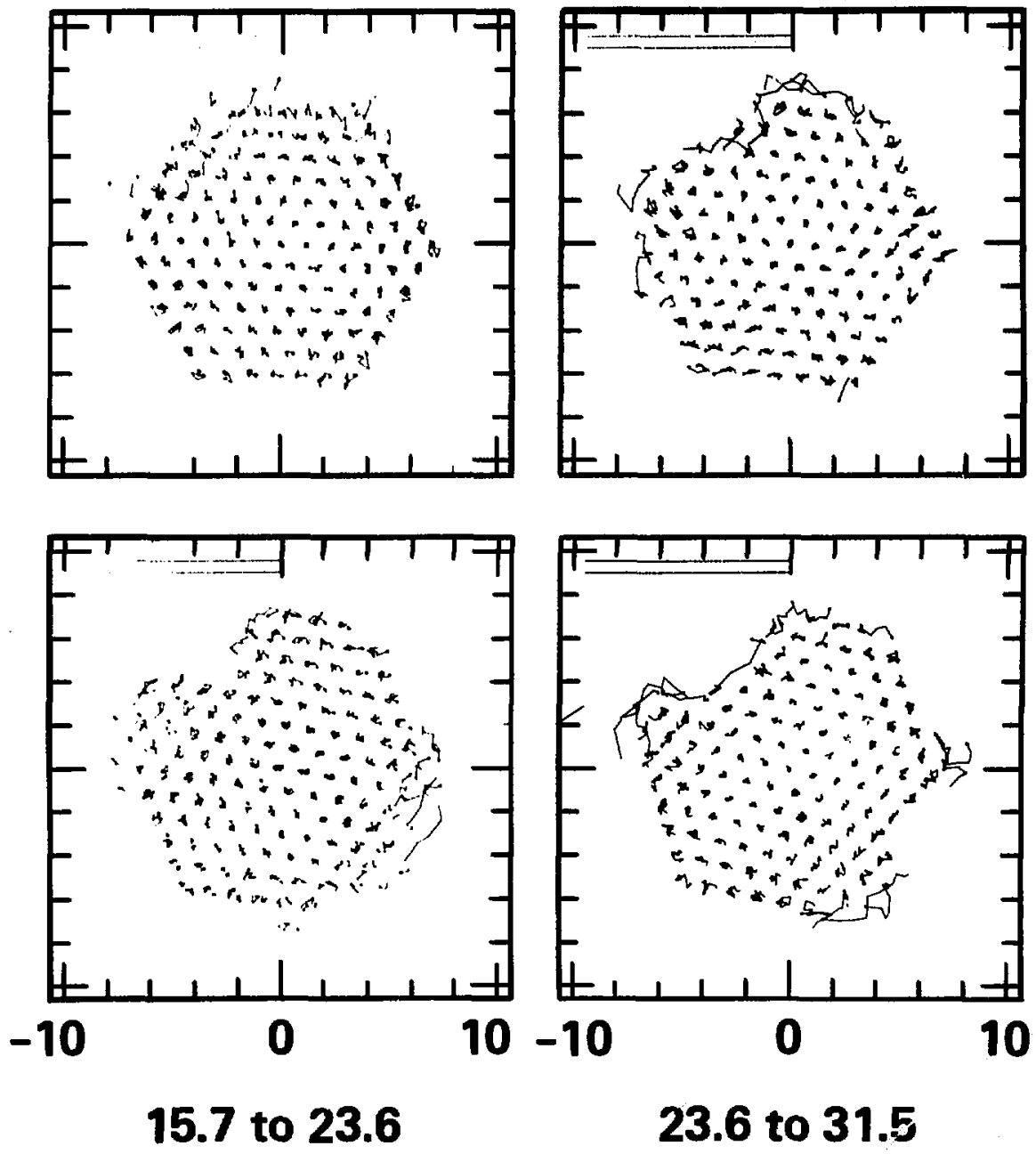

7.9 to 15.7

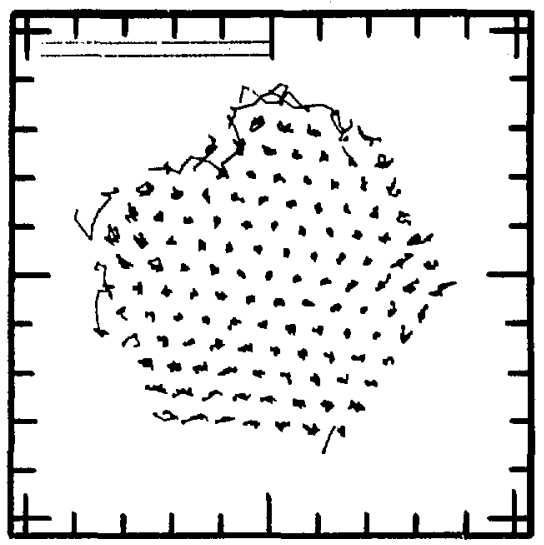

23.6 to 31.5 
runs $C$ and $D$, (liquid and solid-vapor) looked similar because the systems both evolved into the solld-vapor reglon. At a long enough tim, no liquid reglons would renain in these two examples. 
6.5. Establishing the InItial Density Starting with a zoro Temperature Solid

The molecular dynenic fragmentation experiments require en initial circular configuration with the desired density and temperature, and with the potential and kinetic (thormal) onergies being in equilibrium. As a mothod of achieving the correct initial configuration, an easily described expanded-hexegonal solld at zero temperature was allowed to implods from its own surface tension. A surrounding repulsive wall was also converged until the appropriate average density was achieved. Then, the temperature was set by repeatedly adjusting the velocities until equilibrium was established.

The atons were Initially set up as a parfect hexagonal crystal at the appropriate density and with zero temperature (no initial velocities). A redured density of 0.6 was chosen because it is near neither the critical nor the triple point density. At a density of 0.6 , all the initlal bonds are attractive. When time begins, the unbalanced forces on the outer atons (1.e., surface tension) drives a collapsing process (FIg. 6.11 and 6.12). The induced atomic motion Increases the temperature and melting is clearly observable; 1.e., the atoms no longer remain in a fixed arrangenent. To insure the desired final density, the initial hexagon is surrounded by a circular wall with an Inward motion as described below. The potential between the atoms and the wall is purely repulsive and directed toward the array center. 
F10. 6.11. Circularization for a 271 atom system. The initial collepse is driven by surface tension, rather then the moving wall. The wall motion stops when the desired final (circular) area is enclosed.

\section{Time 0 to 5}
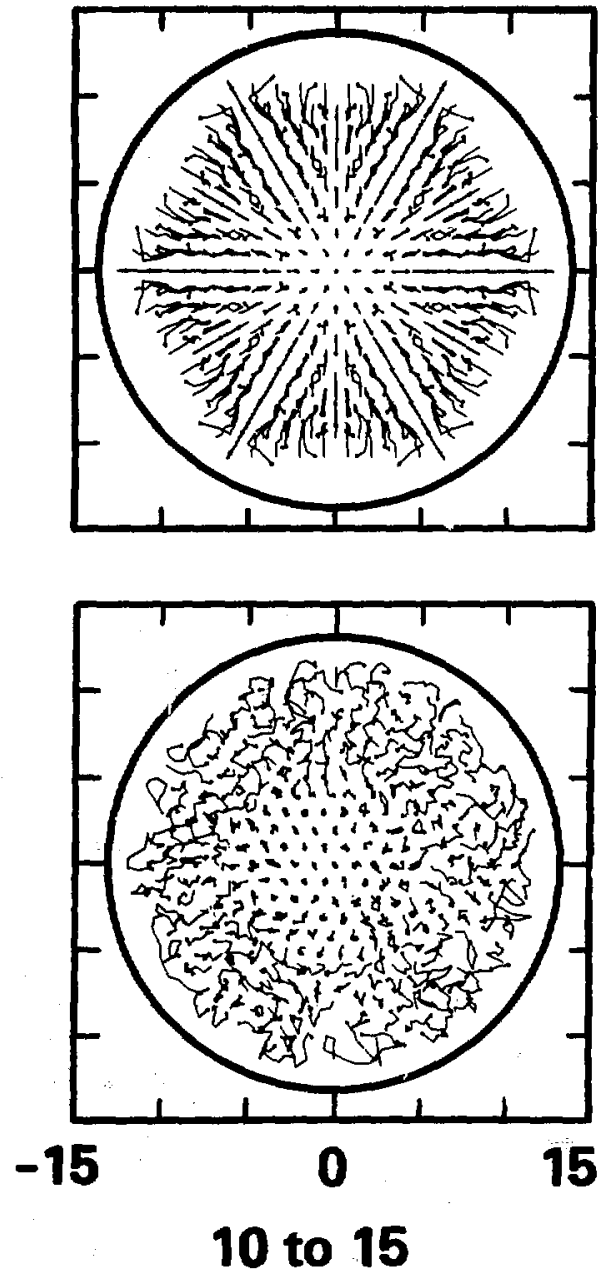

5 to 10
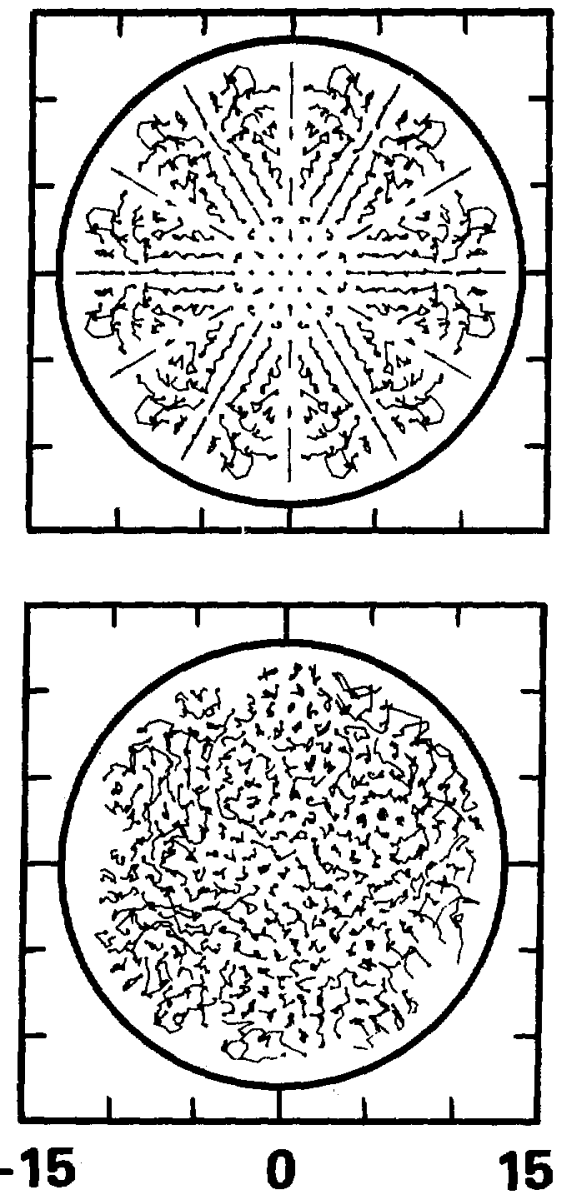

15 to 20 
F1g. 6.12. Circularization for a 2611 atom systcm. The noving mall appears to be thick because it is drawn at several sequential times in each picture.

Time 0 to 6
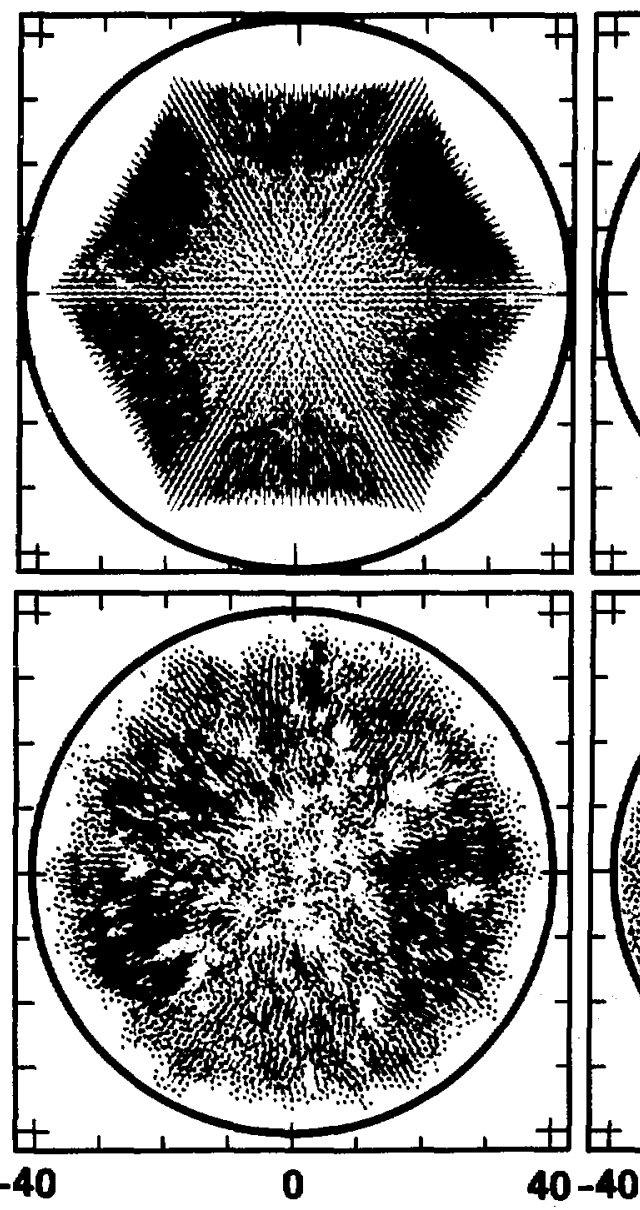

Time 12 to 18
Time 6 to 12
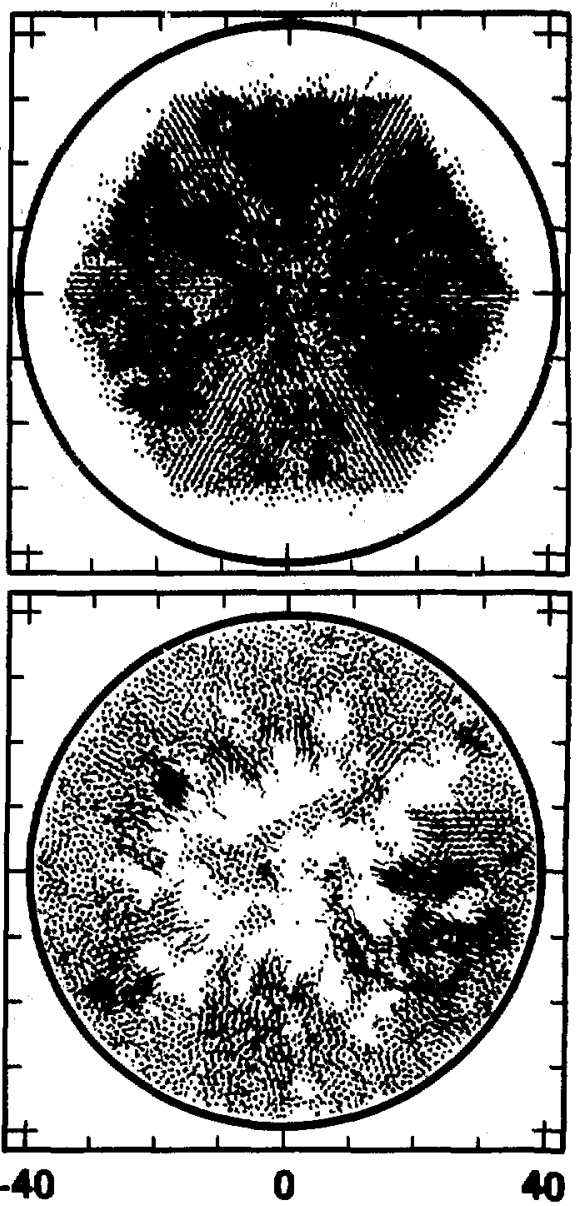

Time 18 to 24 


$$
\begin{aligned}
& \qquad= \begin{cases}4 r^{-12} & , I \leq r_{r} \\
0 & , I>r_{r}\end{cases} \\
& F= \begin{cases}40 r^{-13} & , r \leq r_{r} \\
0 & , r>r_{r}\end{cases}
\end{aligned}
$$

were $r$ is the radial distence betwen the particle and the wall, $F$ is directed radially inward, and $r_{r}$ is the wall-particle force cut off distence. Hre, $r_{r}=d_{0}$, where $d_{0}$ is the initial lattice spacing

$$
d_{0}=1.12246(0.91649 / \rho)^{1 / 2}=1.387 \text { for } p=0.6 \text {. }
$$

In Eq. 6.24, 1.12246 is the rest length (radius at the well depth), and 0.96149 follows from the hexagonal lattice density at the rest length

$$
p \sigma^{2}=2 /\left[\sqrt{3}(1.12246)^{2}\right]=0.91649 \sigma^{2}
$$

The wall prevents any atoms from escaping (as vapor or as fracments) bafore the isochoric heating. During the calculation, the mil slowly moves inward until it reaches the point were the average density within the circle is the desired value. The initial wall radlus is

$$
R_{1}=\left(n_{r}+1\right) d_{0}
$$

where $n_{r}$ is the number of rows in the original hexagonal lattice. The number of atoms in the lattice is

$$
N=1+3 n_{r}\left(n_{r}-1\right)
$$


In Fis. 6.11, the wall is initially one lattice spacing from the lattice while in all other runs, the wall was initially two lattice spacings away. Since the initial motion is determined by the unbalenced forces on the surface atoms (as shom in Figs, 6.11 and 6.12), the initial wall position is not critical.

The outer radius of the final circular array defines the same area as the initial hexagonal array (out to the final aton row, rather than the wall).

$$
r\left(R_{f}-d_{0}\right)^{2}=6\left(\frac{1}{2}\left[\left(n_{I}-1\right) d_{0}\right]\left(\frac{\sqrt{3} d_{0}}{2}\right)\left(n_{I}-1\right)\right)
$$

where $R_{f}$ is the final wall redius. This final wall position is one lattice spacing, $d_{0}$, from the desired outer circle of atoms, and the repulsive force was chosen to be zero for this and larger standoffs. Equation 6.28 can be solved for $R_{f}$,

$$
R_{f}=d_{0}\left[1+\left(n_{I}-1\right)\left(\frac{3 \sqrt{3}}{2 \pi}\right)^{1 / 2}\right]
$$

The magnitude of the wall motion duration was set equal to the number of rows (in reduced time units). The wall velocity can then be found from equations 6.26 and 6.29 ,

$$
v_{w}=-d_{0}\left[0.090608+0.90939 / n_{r}\right]
$$

For 30 rows (2611 atoms), $v_{w}=0.121(230 \mathrm{~m} / \mathrm{s})$. This is much slower than the wave speed measured during the early collepse which was 4.5 $(8600 \mathrm{~m} / \mathrm{s})$. For $500^{\circ} \mathrm{C}$ lithlum the sound speed is $\sim 4500 \mathrm{~m} / \mathrm{s}$.

The tine step was set to 0.01 reduced units $\left(1.5 \times 10^{-15} \mathrm{~s}\right)$ for the circularization calculation. Although this step was a bit small, 
the low number of these calculations dion't merit searching for the largest allowable tine step.

At the end of the circularlzation, the reduced temperature is $-0.54(1650 \mathrm{~K})$ for the 271 aton systom. Thls temperature is in the liquid region on the phase diagram, and the final frome of Fig. 6.11 appears to be a fairly homogeneous liquid. For the 2611 atom system, the temperature after circularization is $\sim 0.7(2140 \mathrm{~K})$. This temperature is also in the liquid region on the phase diagram, but the final frame of Flg. 6.12 indicates a non-equilibrium conflguration. Almost all the atoms are in a ring of liquid that is against the confining wall. If the system continues to evolve with no further wall motion, a homogeneous configuration would eventually be formed, but at a different temperature than 0.7. However, it is better to equilibrate to the desired temperature as described in the next section. The only purpose of the circularization is to produce a circular area containing all the atoms at the correct average density. 
6.6. Temperature Modification and Isochor ic that Mdditisf

The purpose of the equilibration calculation is to produce af equilibrium configuration with the correct avarage density and correct temperature. The non-equilibrium, but correct averege density, configuration produced by the circularization code is the starting point for the equilibration calculation. The circularization and equilibration calculations are linked by a data file written at the conclusion of the circularization calculation. This file contains the positions and velocitles of all the atoms. The two-point velocities are crude since they are based only on the current and previous atom positions,

$$
\vec{v}_{1}=\left[\vec{x}_{1}(t)-\vec{x}_{1}(t-\Delta t)\right] / \Delta t
$$

These crude velocities are adequate to restart the calculation. Splitting the circularization and equilibration calculations allows investigation of numerous starting temperatures with only one circularization calculation. The same technique is used to link the equilibration calculation results to the fragnentation calculations.

The now motionless confining wall maintains the desired average density and prevents fragmentation or vapor pressure generation during thermal equilibration. At each time step, the velocities are adjusted ;o correct the temperature. This procedure changes the calculation from energy conserving to 1sothermal. First, new trial positions are calculated from the original difference equation

$$
\vec{x}_{+}=2 \vec{x}_{0}-\vec{x}_{-}+\vec{F}_{\Delta t} 2 / m
$$

Then, the new and old positions are used to calculate the velocitles, 


$$
\vec{v}=\left(\vec{x}_{+}-\vec{x}_{-}\right) / 2 \Delta t
$$

Next, the velocities are adjusted to produce the correct temperature,

$$
\vec{v}=\vec{v}_{0}\left[2 K /\left(m\left[v_{0}^{2}\right)\right]^{1 / 2}\right.
$$

where $K$ is the desired kinetic energy, NkT. The adjusted velocitles are used to influence the particle notion by correcting both the old and the new positions $\left(\vec{x}_{-}\right.$and $\left.\vec{x}_{+}\right)$, although the correction in $\vec{x}_{-}$ is not directly seen since that information has already been output at the time of the adjustmont. The adjustment is straight forward. Equations 6.32 and 6.33 are used to eliminate $\vec{x}_{-}$, and the resulting equation is solved foi $\vec{X}_{+}$,

$$
\vec{x}_{+}=\vec{x}_{0}+\overrightarrow{v \Delta t}+\vec{F} \Delta t-/(2 m)
$$

The equilibration calculation used to start the Isochoric heating celculation for the 2611 system evolved into the procedure described above. In the original calculation, the post-circularization configuration was allowed to equilibrate for 128 reduced time units (6000 time steps). However, the temperature was only adjusted once every 500 steps. The desired temperature was 0.55 , and the adjustments were designed to overshoot this mark to attempt to anticlpate equifartition of added or removed energy between the kinetic and potential energles. This procedure was not perfect, and the temperature oscillated around the desired value (Fig. 6.13).

For a density of 0.6 , the phase diagram (Fig. 4.6) indicates a saturation temperature of 0.53 . The isothermal model with temperature adjustments at every time step was started from the 6000 step 
F19. 6.13. Temperature oscillation during the initial 6000 step equilibration (18.6 ps).

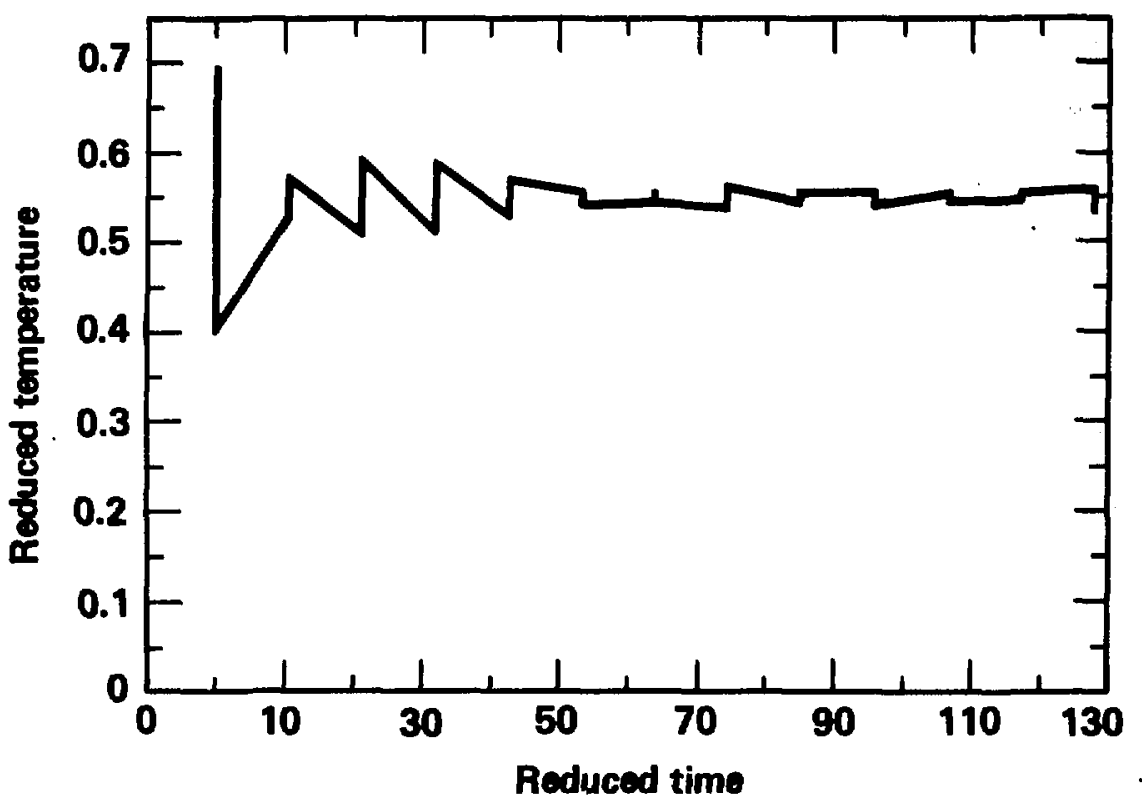


conflguration (FIg. 6.14). Five isothermal equilibration calculation were run for 1000 time steps each ( -28 tine units) for temperatures bracketing the saturation temperature $(0.42,0.45,0.5,0.55$ and 0.55). The final configurations for these five calculations are shown in flgures 6.15-6.17. The low temperature results are clearly in the two phase region with outer gas pockets surrounding central liquid. The higher temperature results appear to be pure liquid although volds of up to $\sim 50$ in diameter are scattered within the llquid and at the edges. Fehder 27 also saw snall volds in molecular dynanics studies of liquids at $\rho, T$ conditions of $(0.68,0.93),(0.68,1.44)$, and $(0.46,1.15)$.

The small volds could indicate that either the system is in the two phase liquid-vapor region or it has not yet reached mechanical equilibrium (that is, more equilibration time is needed). To test the first possibility, an equilibration calculation was run at a very high. reduced temperature, 1.153, which is above the critical point. The configuration after 3000 time steps (11 ps) is shown in Fig. 6.18. Even at this high temperature, small voids exist within the liquid. This result is analogous to Thompson's study of small drops ${ }^{29}$ wich showed that drops are non-spherical at any instant of time, but the tim average of the drop shape is spherical. In the two-dimensional 1iquid, instentaneous voids exist, but the time average of the density at any location is the bulk density.

The possibility. that the voids are symptomatic of an uncquilibrated system cen also be dismissed. In Fig. 6.19, the potential energy per atom is plotted as a function of time for the wilibration calculations. In each case, constant potential energy 
Fig. 6.14. Configuration at the end of 6000 equilibration time steps (18.6 ps).

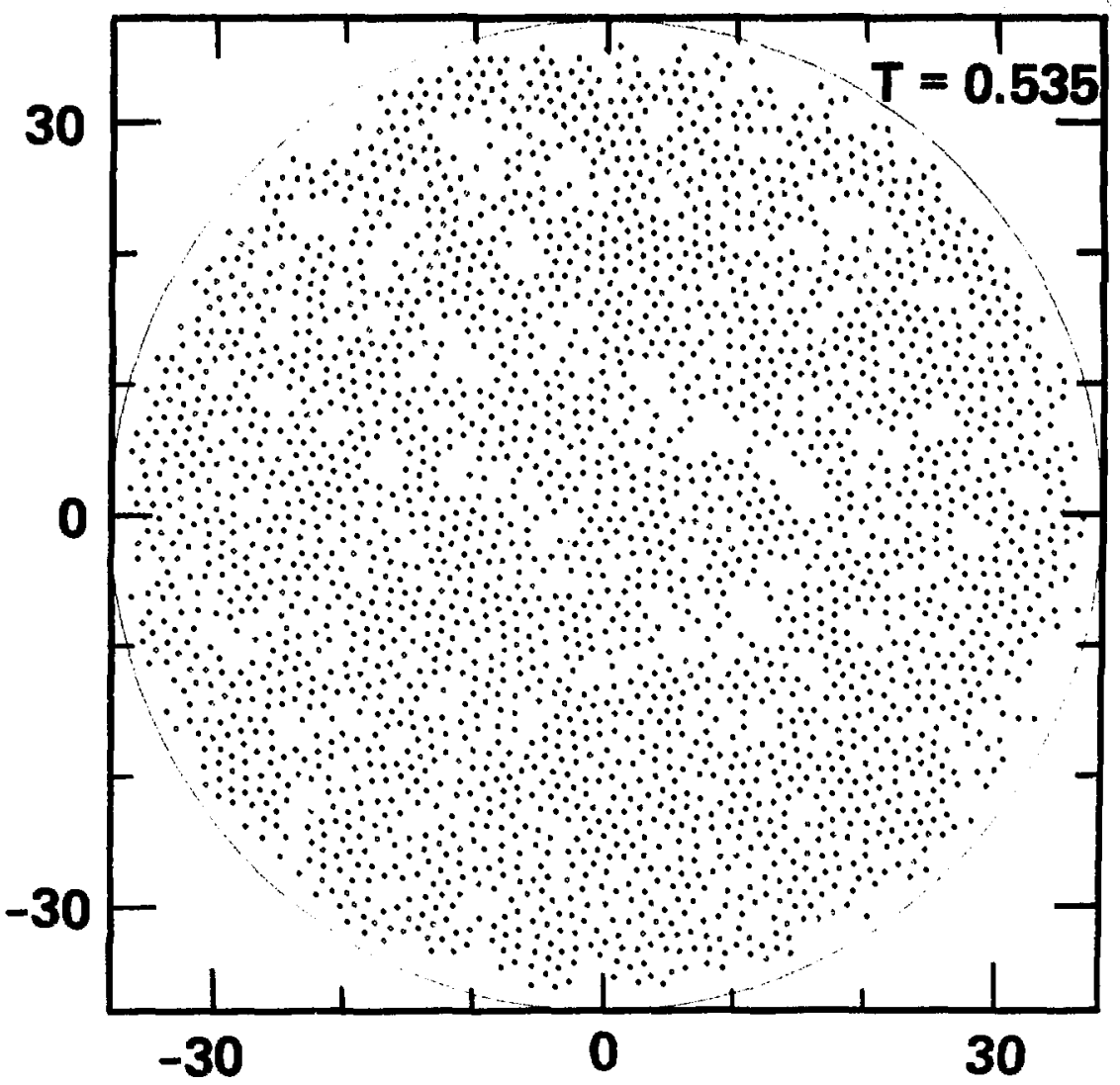


Fig. 6.15. Final configuration after 1000 isothermal time steps ( $\sim 4.4$ ps) at temperatures of 0.42 and 0.45 .

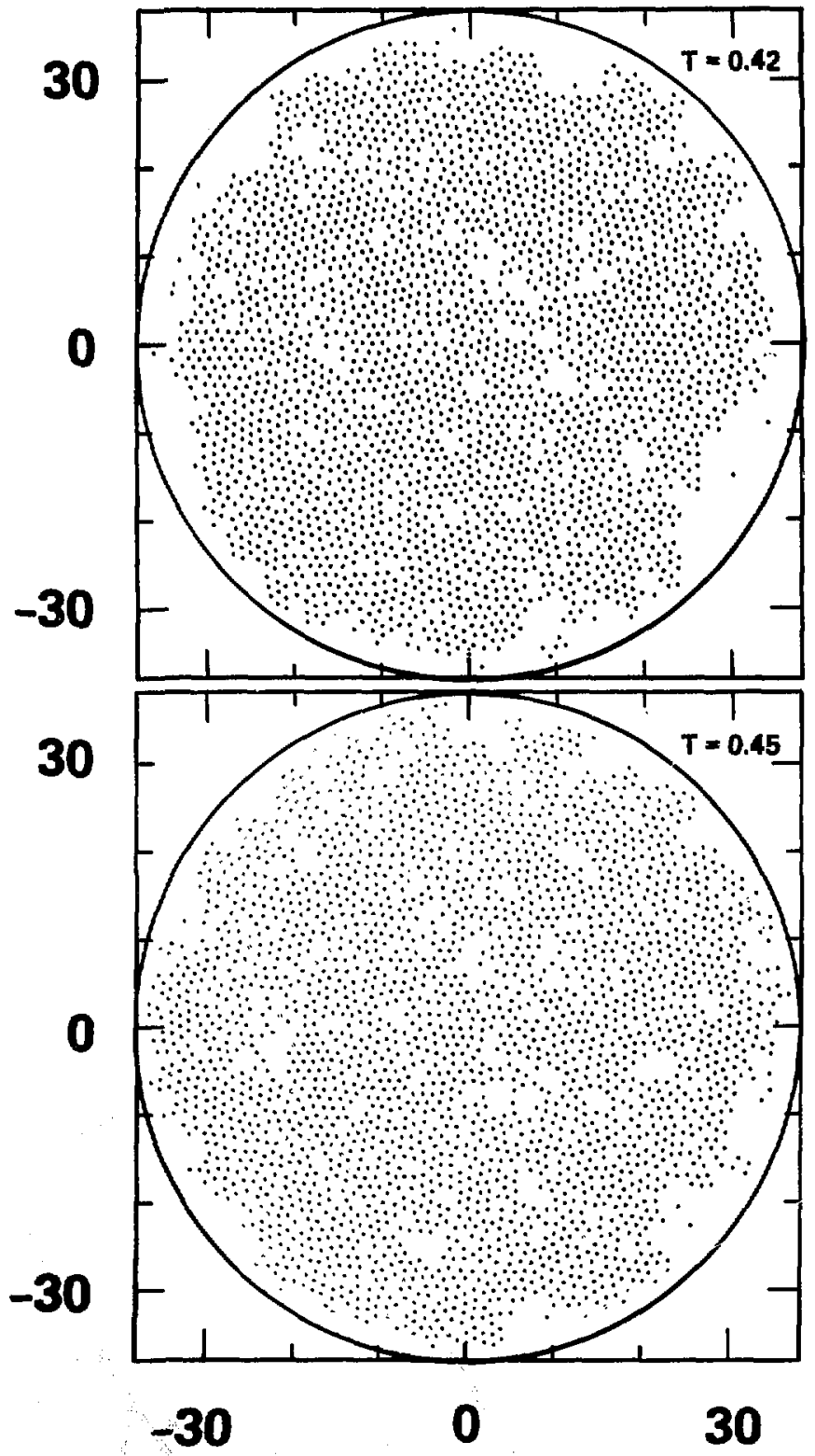


Fig. 6.16. Final configuration after 1000 isothermal tim steps ( $\sim 4.4 \mathrm{ps})$ at temperatures of 0.5 and 0.53 .

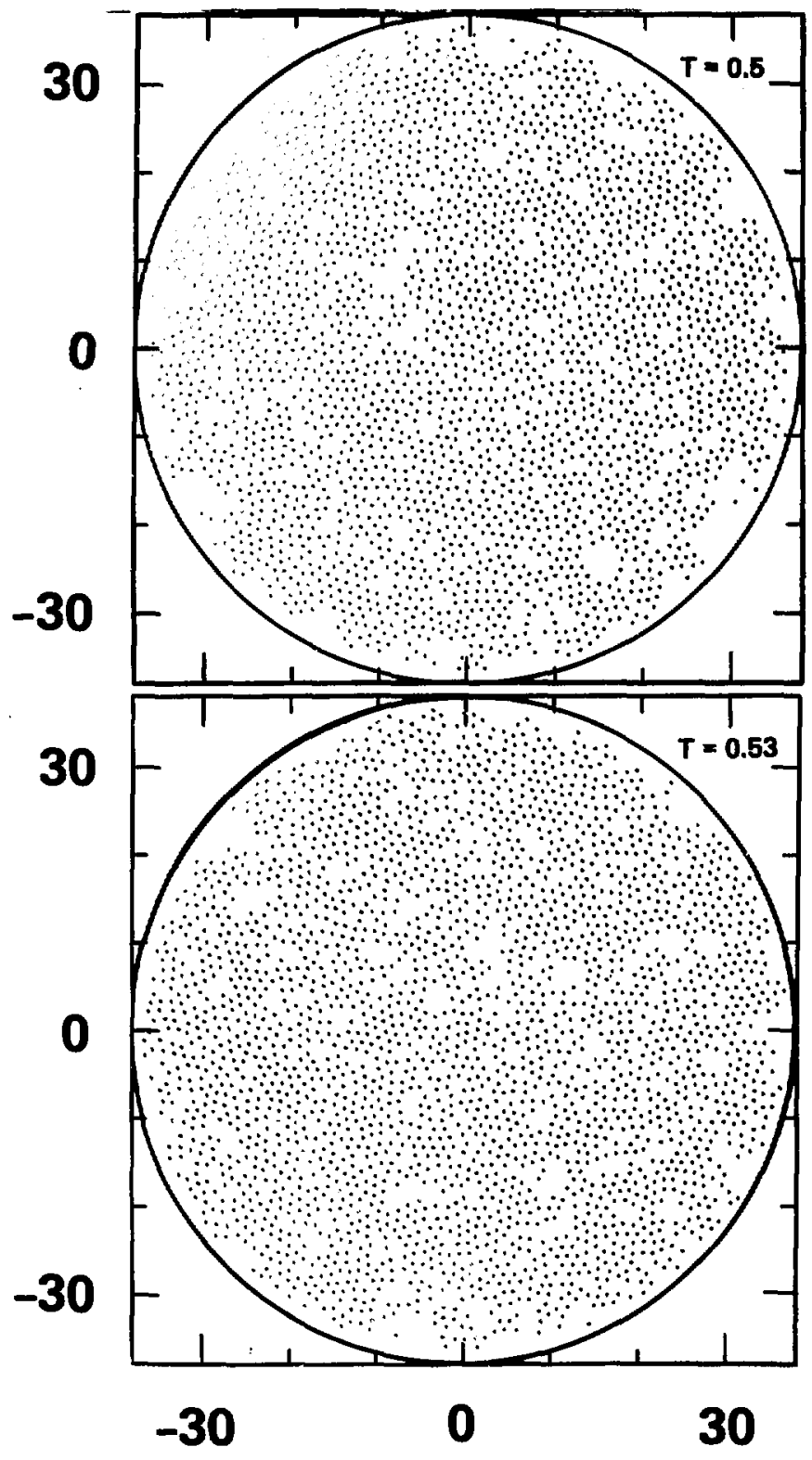


Fig. 6.17. Final configuration after 1000 isothernal time steps

$(\sim 4.4 \mathrm{ps})$ at a temperature of 0.55 .

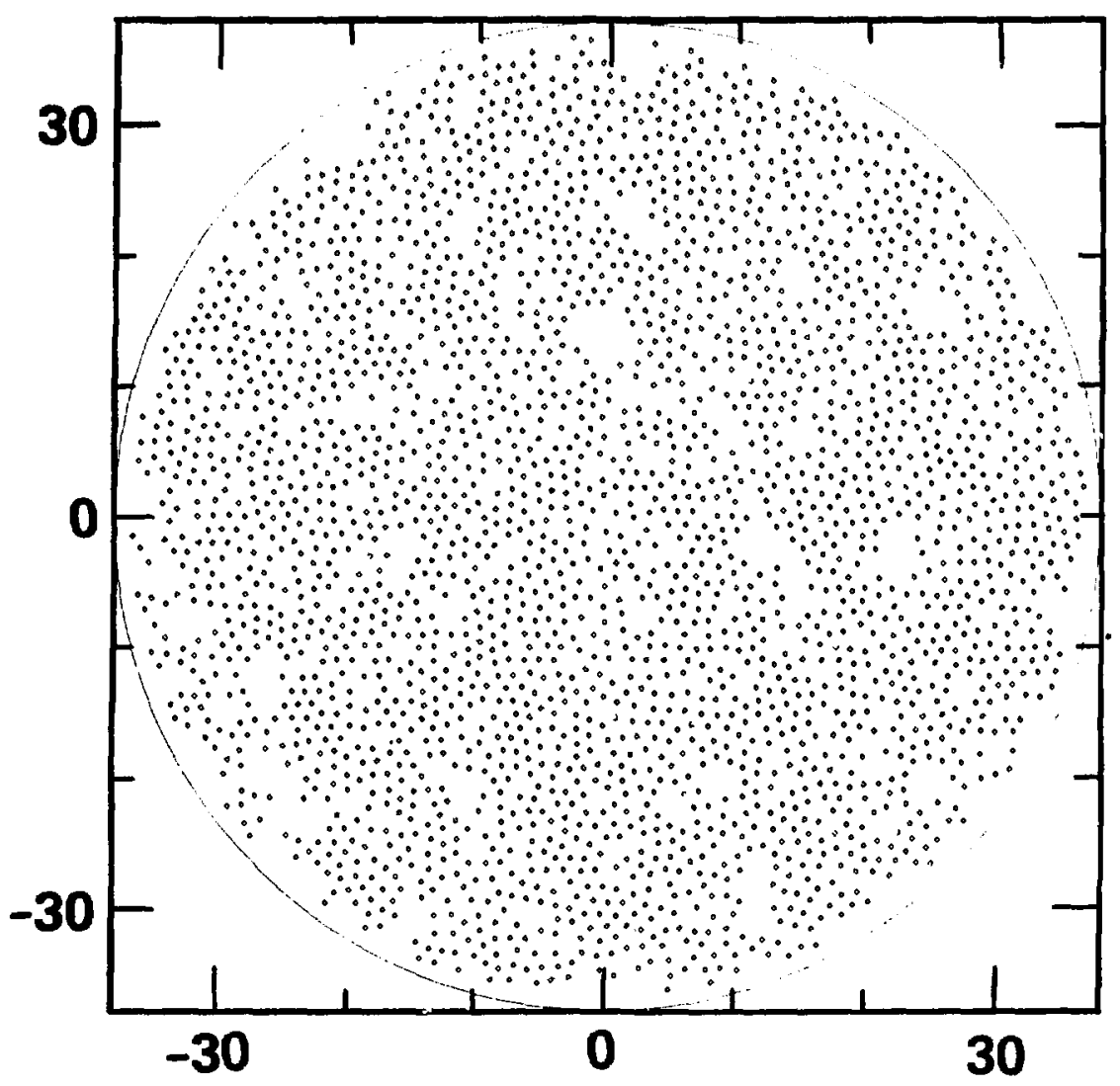


Fig. 6.18. Configuration of 721 atom system equilibrated to a temperature of 1.153 for 3000 time steps (11 ps).

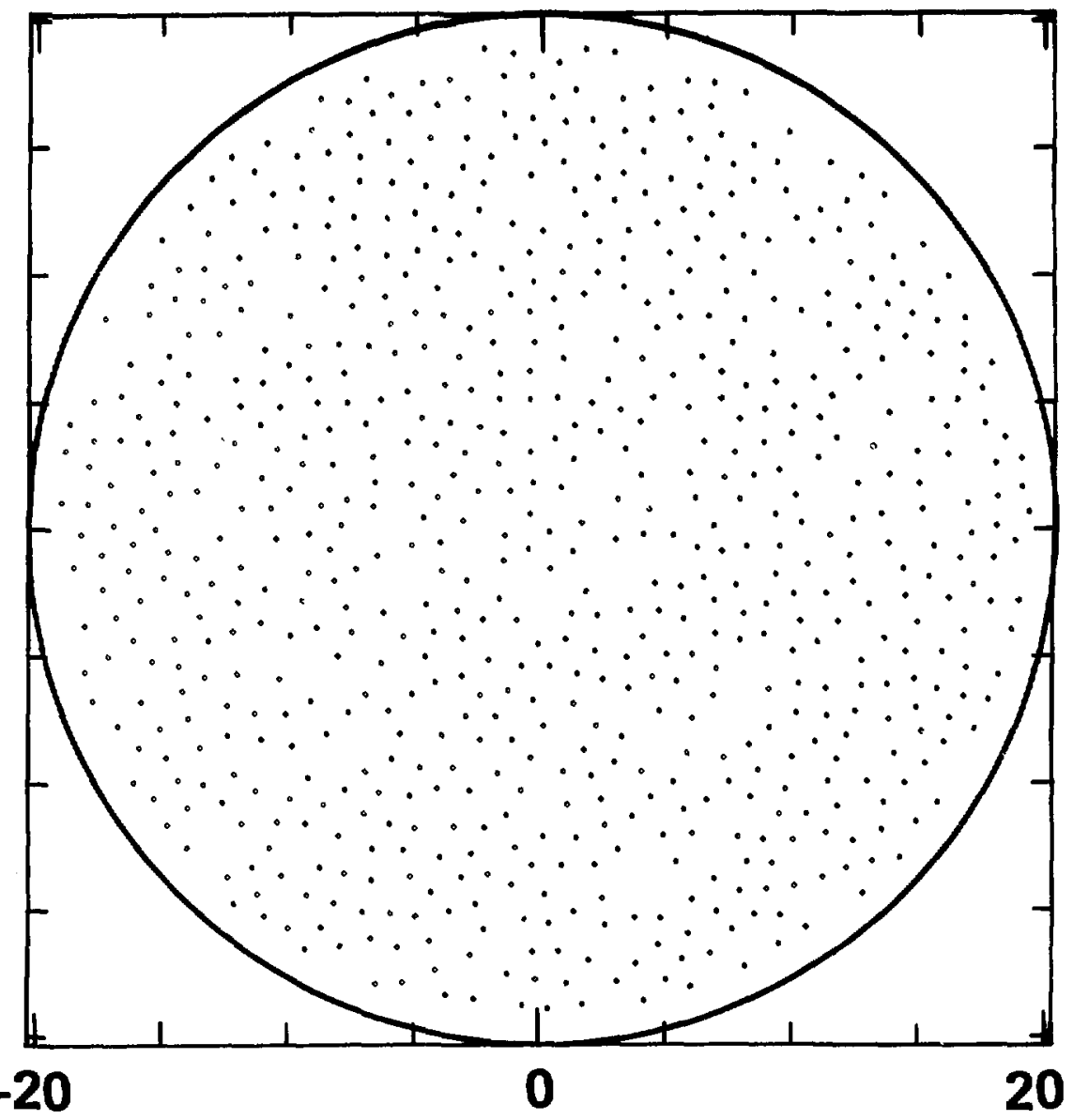


F1g. 6.19. Potential energy per atom as a function of time for seven equilibration runs. Mechanical equilibrium seems to be reached at -10 time units (1.5 ps) in each case. Solid lines are for 2611 atom systems while dashed lines indicate 721 atom systems.

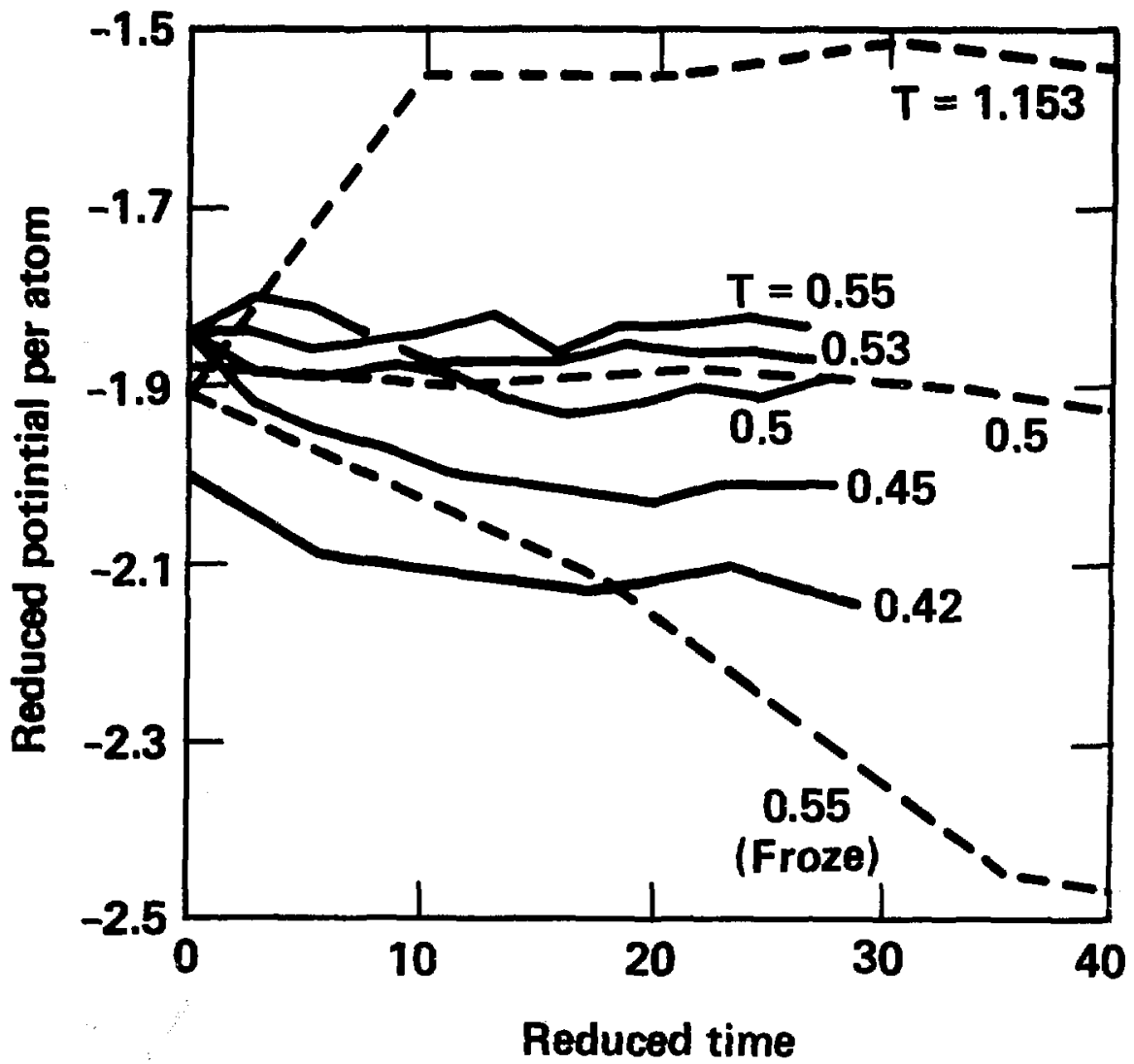


(mochenical equilibrium) is reached in 20 tim units (1.5 ps). The decreasing potential energy manitude $(|\Sigma \$ / N|)$ with increasing temperature can be attributed to either fower bonds or to less energy per bond. For the 2611 atom runs in the 0.42 to 0.55 temperature range, the change was almost entirely du to the change in the number of bonds. For the 721 atom runs (at tmperatures of 0.5 and 1.153 ), the change in potential energy per atom was due to roughly equal contributions from fewer bonds and less energy per bond.

Thus, it appears that the small volds are real. Thelr existence will influence the fragmentation process and the molecular dynamics simulations. Fragmentation in ICF reactors will produce fragments that are much larger than the vold spacing, and the molecular dynanics experiments should be designed to produce fragments that contain at least several voids. That is, fragments smaller than -150 atoms should not be trusted since their shapes and sizes will be primarily determined by the individual voids rather than the hydrodynamics. For larger fragnents, the statistical distribution of the volds wlll be more important than individual volds.

The 1sothermal equilibration worked well for most of the systems . studied. However, it is possible to misuse this technique. For example, a 721 atom system was equillibrated to $T=0.5$, and the resulting configuration appeared to be two-phase liquid-vapor. A further equilibration to $T=0.55$ was attempted in order to nove vertically into the liquid region on the T-p diagram. Instead, the system followed a path up and to the right, all the way into the solid region, as indicated by the frozen structure in Fig. 6.20 which appears to have about a half-dozen nuclei and interesting crystal 
Fig. 6.20. Final configuration of a 721 atom system that froze during temperature equilibration.

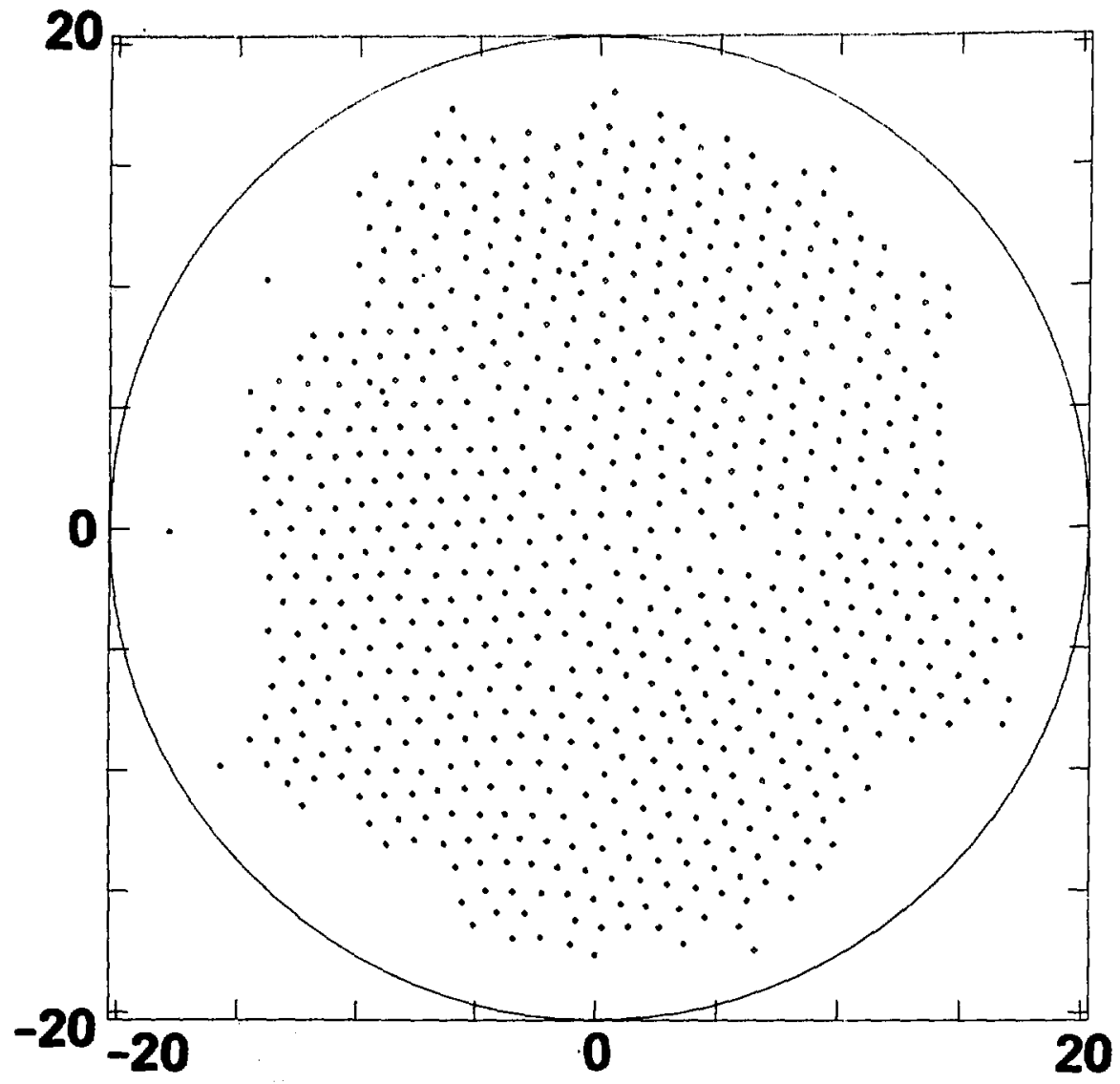


grains. The potential enorgy per atom is -2.5 compared a vilue of -1.8 for the liquid in Fig. 6.17 wich is at the semperature; the large decrease is consistent with freazing as the atoms assime their closer spacing deeper in the potential well. Evidently, the response to each adjustmont increased both tcmparature arif censity, causing further adjustments to continue the process. This sequence of events could have been caused by a radial oscillation of the system, 1.e., if most of the atoms happened to be noving imward at the beginning of the equilibration run, increasing density would be expected.

The isochoric heat addition was input by specifying a heated temperature and adjusting the velocities (and hence the temperature) using Eq. 6.33. In early runs, an attempt was made to compensate for the overshoot due to equipartition (conversion of a portion of the added kinetic energy into potential energy). However, the actual temperatl e immediately following the energy addition is available in the output, and that value was used wren the runs were evaluated in Chapter Seven. In later runs, the disired heated temperature was produced without overshoot.

Since the velocity adjustment procedure multiplies an already equilibrated velocity distribution, it is also equilibrated. However, the atoms are not spatially equilibrated for about -10 tine units. Since this time is of the same order as the wave transit sime across the systen, the equilibration calculations were rerun at the temperature desired after isochoric heating. These results provide the Initial conditions for the fragmentation runs which begin when the confining wall is suddenly removed.

The actual energy distribution in an ICF reactor is dependent on 
$-154-$

the neutron moderation and absorption processes, and is probably highly non-uniform. Yet, in the macroscopic fluid, the added energy reaches spatial and velocity equilibrial in a time short (sub-ns) compared to either the energy addition time (us) or the pressure relief time ( 40 ps in HMIFE). Thus, the microscopic fragmentation runs should start from configuration equilibrated at the heated temperature (rather than the saturation temperature). The results discussed in chapter seven all begin from configurations equilibrated at the heated temperature. 


\subsection{Grephic Output}

Although a voluminous data output cen be produced by ecch run (as described in the next section), a graphicel dieplay allowed quick evaluation of the results and also provided hints as to wich numerical data would be most interesting. Four types of grephical output were produced during the evolution of the molecular dynanics nodels.

- Early runs used frequent snapshots of the atomic positions, with bonds indicated by lines connecting the atoms. When run as film strip, this output allowed easy visualization of both tensile and compressive waves.

- Later film strips eliminated the bond lines; this also proved to be effective. A complete film sequence of the circularization, equ!librium, and fragmentation of a 2611 atom system was produced as a part of this work. The author is willing to lend a copy of the film upon request.

o Because producing many frequent frames is very expensive, a time-exposure that showed the trajectories of the atoms for tens and even hundreds of time steps proved to be quite economical. The trajectories were dram about ten times per frame, 1.e., the motion of an atom for a few to a few tens of steps was drawn as a single straight line. In practlice, this procedure saved considerable computer tine while preserving an adequate amount of trajectory information. At the end of each time exposure freme, a simall diamond was drawn at the end of each trajectory; this allowed determination of the directions of the trajectories. 
o The most economic grephic output orew diamonds at the atom locations once per frawe (no trajectories wore dramn). Even this ilmited grsphic output proved to be quite useful. 
$-157-$

6.8. Tabular Output

At selected time steps during circularization, equilibration, and fragmentation, data were written to en output file. These results fell into two categories: data summarized over all atom e and data summarized by cluster (fragment). Data output included bond count, potential and kinetic energies, translational and rotational energies, temperature, density and PV product. 


\subsubsection{Data Summrized Over All Atoms}

At selected time steps, a number of overall quantities were computed and sent to the output data file.

- Bond Count - During the calculation of the interatomic forces, the bond count was incremented whenever the interatomlc separat'on was less then the cutoff distance (40 for early calculations and 2.50 for later calculations). The number of bonds In the system decreased when the density decreased and when atoms were - jected as vapor pressure.

D Kinetic Energy - The kinetic energy in each coordinate direction was computed.

o Potential Energy - The total potential of all the bonds was calculated at the same time as the forces. The potential was also outjut as the delta potential above the perfect potential. The perfect potential is defined as the potential energy for a perfect hexagonal crystal when all the first neighbor interatomic separations correspond to the bottom of the potentlal well, 1.e., when $2 \phi / 2 r=0$ or $r_{0}=1.122460$. The potential energy increased during circularization and when particles were ejected from the systcm. For a completely vaporized state, the potential would be epproximately zero since virtually no atons are within the bond range at any given timo.

D Total Energy - During the fragmentation calculations, conseriation of the sum of the kinetic and delta petential energies to within $2 x$ of the initial value was required. The energy quantity chroked was the sum of the kinetic and delta-potential energles to wotd problems men the actual energy is small and negative. Poor 
energy conservation was usually due to the choice of en excessivaly long time step. When energy conservation wa a problem, the velocities were adjusted or the time step was dacreased. For the circularization and equilibration calculations, eneroy was not rigorously conserved because of work done by the wall and because of velocity adjustments used to correct the temperature.

- Wall Data - During circularization and equilibration, the wall interacted with the outer particles. At output tims, the code totaled the wall-atom bond forces and potentials. The pressure on the wall was also computed by dividing the force (which is radial) by the wall perimeter. This two-dimensional pressure has units of force per unit. length or, equivalently, energy per unit area. 


\subsubsection{Data Sumarlzed by Cluster}

A cluster is defined as set of atoms that have bonds with each other but with no other atoms. In this study, the potential was truncated at either 2.50 or 4.00 , allowing straight forward determination of the cluster identities when the forces were calculated. First, oll atoms wre assigned to cluster number $(N+1)$ where $\mathrm{N}$ is the number of atoms in the system. Then the nested do loops that calculate the forces ware entered. The outer do loop Incremented the first atom, $N_{1}$, from 1 to $(N-1)$. The Inner do loop incremented the second atom identification, $N_{2}$, from $\left(N_{1}+1\right)$ to $N$. On each cycle of the outer do 100p, the first atom in the atom pair was assigned to its own cluster. On each cycle of the inner do loop that calculated a non-zero bond force, the second atom was assigned to a cluster. If its current cluster number was $(N+1)$, it was assigned to the same cluster as the first atom. If the second atom was in neither cluster $(\mathrm{N}+1)$ nor the same cluster as the first atom, the maximu $\left(N_{\max }\right)$ and minimum $\left(N_{\min }\right)$ of the cluster assignment of both atoms were calculated. Then the cluster assignments of all the atoms were reviewed, and atoms in cluster $N_{\max }$ were reassigned to cluster $\mathrm{N}_{\text {min }}$.

After the nested do loops were completed, the cluster assignments were reviewed. First, atoms with no assignment were assigned to new clusters. Then, the population of each cluster was calculated. Because of the reassignment procedures, some cluster numbers had zero population. Hence, the cluster assignments were revlewed one last time to edjust the cluster numbering to count only non-zero population clustars. 
Once the cluster assignments were complete, the center of mass, translational energy, rotational energy, thermal energy, and temperature of each cluster were calculated. The values of these quantities were calculated simultaneously for each cluster. This was accomplished by looping through the atom list (index $j$ ) and incrementing the quantity, $f\left[N_{1}(j)\right]$, where $f$ is the quantity of interest and $N_{1}(j)$ is the cluster assignment (1) for atom $f$.

The center of mass for a given cluster is $\langle\vec{x}\rangle=(\Sigma \vec{x}) / N_{c}$ where the sum is over the $N_{c}$ atons in the cluster. In this and the following cluster quantity formulas, the atomic mass, $m$, was lgnored since it was set to 1.0 .

The cluster kinetic energy is $\Sigma v^{2} / 2$. The cluster transiational energy can be found from either the motion of the center of mass or the average velocity. Using the latter method,

$$
\vec{v}=\Sigma v / \vec{N}_{C}
$$

and the translational energy is

$$
N_{c} v+2=(\Sigma v) \rightarrow 3(2 N)_{c}
$$

The cluster rotational energy is $L 2 / 2 I$. The angular momentum is

$$
L=\Sigma\left[(x-\langle x\rangle) v_{y}-(y-\langle y\rangle) v_{x}\right],
$$

and the moment of inertia is

$$
I=\Sigma(\vec{X}-\langle\vec{x}\rangle)^{2}
$$

The thermal energy of the cluster was found by subtraction since it includes all the kinetic energy except the center-of-mass translation and rotation. The cluster temparature was found by dividing the thermal energy by $\left(N_{c} k\right)$. 
The cumulative density was calculated as a function of radial distence from the atom nearest the center of mass of each cluster containing more than 20 atoms. For these clusters, a cumulative density profile.

$$
\left[\Sigma(i)_{\left.\left(r_{1}\right) r_{\rho}\right)}\right] / \pi r_{p}^{2}
$$

was plotted in radial increments of $0 / 4$.

The virlal theorem was used to calculate PV for each cluster

$$
P V=\frac{1}{D}\left[\sum v^{2}+\underset{1<j}{\sum}\left(\vec{r}_{1 j}+\vec{F}_{1}^{j}\right)\right]
$$

where $D$ is the number of dimensions, $\vec{r}_{1 j}$ is the vector between atoms 1 and $j$, and $\vec{F}_{1 j}$ is the force on atom 1 due to atom $j$. The right hand side of the equation should be time averaged; however only instantaneous values were calculated at energy edit times. Time averaging was done afterwards for the limited number of energy edits.

Once all of the above cluster quantities were calculated and output, they were sumed and output for the entire system. 


\subsection{Computational Efficiency}

Once the computer cades were tested for a small system (127 atoms), the run tine was andyzed and extrapolations wre made to see what size system could be reasonably analyzed. Then, methods of increasing the calculational efficiency were considered.

For the test Iun, the everage step time on the CRAY-1 computer was $19 \mathrm{~ms} / \mathrm{step}$. This calculation considered all atom palrs at each time step. Most of the run time was used in the mested do loops that calculated the interatonic forces. Because these loops contained "If" statements to clrcumvent part of the loop when the interatomic separation was larger than the cutoff distance, they would not vectorize. Vectorized do loops run up to an order of magnitude faster than non-vectorized loops.

An easy way to vectorize the nested luops is to eliminate the bond cutoff and calculate the forces for all atom pairs. This increases the number of bonds by a factor of five for the 127 aton system. The average step time for the fully vectorlzed code was 54 ms/step, indicating that this tactic would not be useful, particularly for larger systens.

For the non-vectorized code, another run was made for a 271 aton system. Comparison of memory usage indicated that a 120,000 atom system could be calculated with $85 \%$ of the machine's mewory. Unfortunately, the run time for such a large system would be unreasonable. If run time were proportional to the system popuiation, over $50 \mathrm{CPU}$ hours would be required to study a 120,000 atom system. It is likely that considerebly more time would be required since run time increases faster (as $N$ ln $N$ ) than the system population. Hence, a 
major improvenent in calculational efficiency was required.

One of the earliest methods of increasing calculational efficiency, sugoested by Alder ${ }^{53}$, was to infrequantly compute the projected collision times, if any, of all possible palrs of atoms. Then, the tim step was adjusted after each step to cover the projacted time until the naxt collision. In the same publication, Alder suggested dividing the region into grids and only consider atons pelrs in nearby grids; this was the precursor to nelghbor 11sting. In 1967, Verlet ${ }^{54}$ introduced a neighbor listing scheme (called Verlet tables by some authors). Recently, Thompson ${ }^{55}$ has optimized and automated neighbor listing. In Thompson's method, a list is constructed for each atom that includes all atons within some range $I_{2}$, of the atom. The range must be larger than the potential's truncation renge, $r_{t}$, and it should be smaller than the radius of the system. Once the list is established, only those atoms on the list are considered when calculating the interatomic forces. The greatly reduced set of atom pairs to be considered at most time steps correspondingly decreases the run time. Conversely, the neighbor list array is large, between $N$ and $N^{2}$, depending on the cholce of $r_{\mathbf{l}}$. The nelghbor list becomes invalld when the atons have moved far enough that they have interactions with cther atoms not on the list. The list Ilfetine depends on the atomic motion in Thompson's scheme. when the neighbor list is built, en array that has dimension equal to the number of atom is set to zero. After each time step, the array entries are increasud by the scalar distance traveled by the atoms. Before the next tine step, the maximum of the distance array is compered to a test quentity. If the maximm distance is less then the 
test quentity, enother time step using the current nelobor list is allowed. The test quentity is $0.95\left[1-1 /\left(n_{1}+1\right)\right]\left(r_{1}-r_{t}\right)$ where $n_{l}$ is the number of time steps the list has been used. The variable test quantity enticipates whether the naxt step will move the atoms past the list range. The enticipation is based on the number of steps already accomplished since the last list update. In this study, the total path length was used since veporizing atoms travel in straight paths. In a more confined system, it would be better to consider the net distance traveled, allowing use of the list for longer periods.

Thompson tested the neighbor list procedure for three dimensional 256 and 500 atom systems. 55 the used a reduced density of 0.8 , a reduced temperature of $0.76,1000$ time steps, and a Lennard-Jones potential truncated at 2.50. In both cases, he found that the step tine sharply increased for list redil less than 2.60; this was because the list was updated too frequently. Thompson also found a gradual rise in step tine for list radil above 2.90; this was because the list became excessively long, and time was wasted on each step in checking too meny non-Interacting atom pairs. Thompson's optimum list radil were 2.70 (2.1 CPU seconds per step) for the 256 atom system arid 2.80 (4.4 CPU seconds per step) for the 500 atom system. His data was collected using a PDP 11/70 minicomputer that has approximately $18 \%$ of the speed of an IEM $370 / 168$.

The nelghbor list procedure was integrated into the computer prograns developed in this study. The step time is shom as a function of the l1st radius for a 2611 atom system in Flg. 6.21. The optimum list radius is about $4.0 \sigma$, and the step time is in the 220 
F1g. 6.21. Step time dependence on list radius for a 2611 atom system.

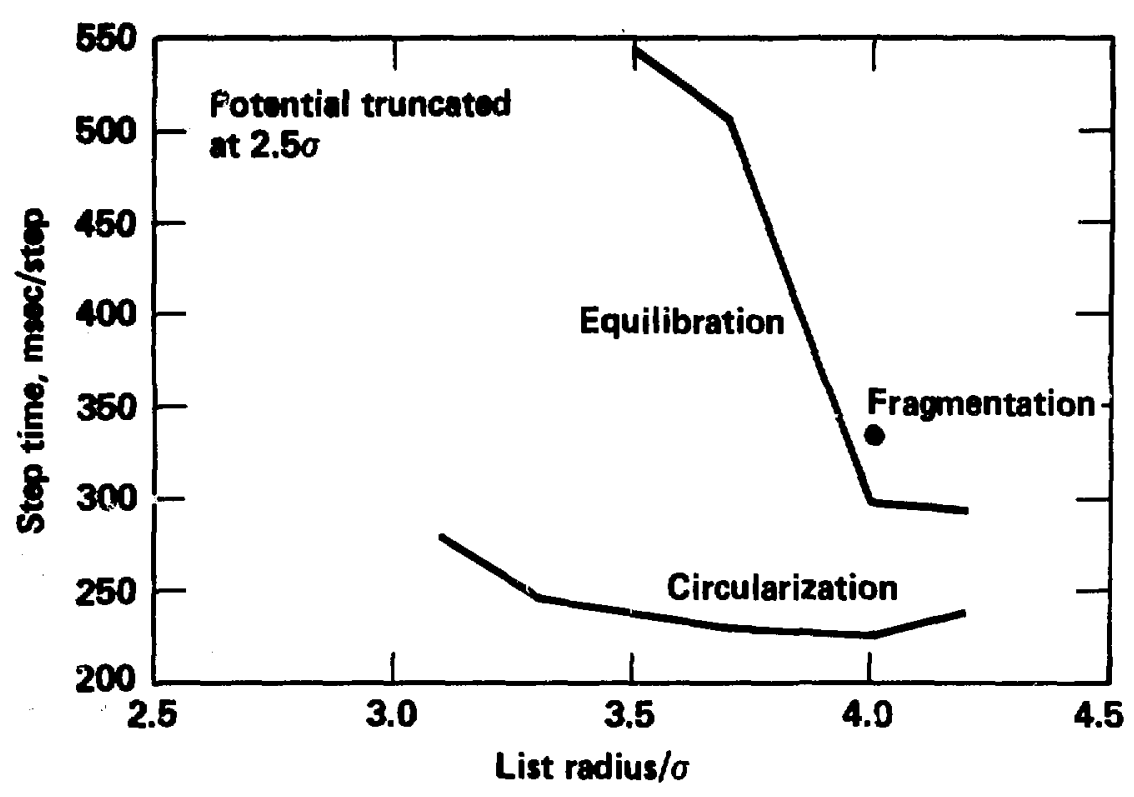


to $330 \mathrm{msec} / \mathrm{step}$ range depending on the type of calculation. For comparison, without nelghbor listing, the circularization calculation required $1.5 \mathrm{sec} / \mathrm{step}$. The circularization calculation was the fastest because it had a minimm of atomic mixing wile it was solid. Figure 6.22 shows the list update frequency as a function of the list radius. Equilibration and fragmentation calculations used each list for about 25 time steps inlle circularization meighbor 11sts ware valid for about 120 time steps. The momory price paid for the Increased speed is shown in Fig. 6.23 wich plots the neighbor list array size as a function of the 11 st radius. An array of about 40,000 d'

Because it takes longer for waves to traverse large systems, the 2611 atom calculations require more time steps thar ine smaller systems. A typical problem required 19 minutes of CPU tins during circularization, 37 minutes during equilibration, and 24 minutes during fragmentation.

The step time, list size, and list update inuervals are surmarized in Table 6.2 for fragmenting 169, 721, 2611, and 14491 atom systens. The $6.7 \mathrm{~ms} / \mathrm{step}$ time for the 169 atom system was considerably better than the $19.7 \mathrm{~ms} / \mathrm{step}$ measured in the smaller 127 atom test run without neighbor listing. Although it was unreasonable to run a test problem without. neighbor listing at 14491 atoms, the improvement at that size was probably many orders of magnitude. Even *ith nelghbor listing, computer time rather than memory placed the system size linit at $\sim 15,000$ atons (10.5 Cray CPU hours were consumed by the single 14491 atom fracmentation run, but less then 1/6 of the available nemory was used by the code.) 
Fig. 6.22.: List update interval for a 2611 atom system.

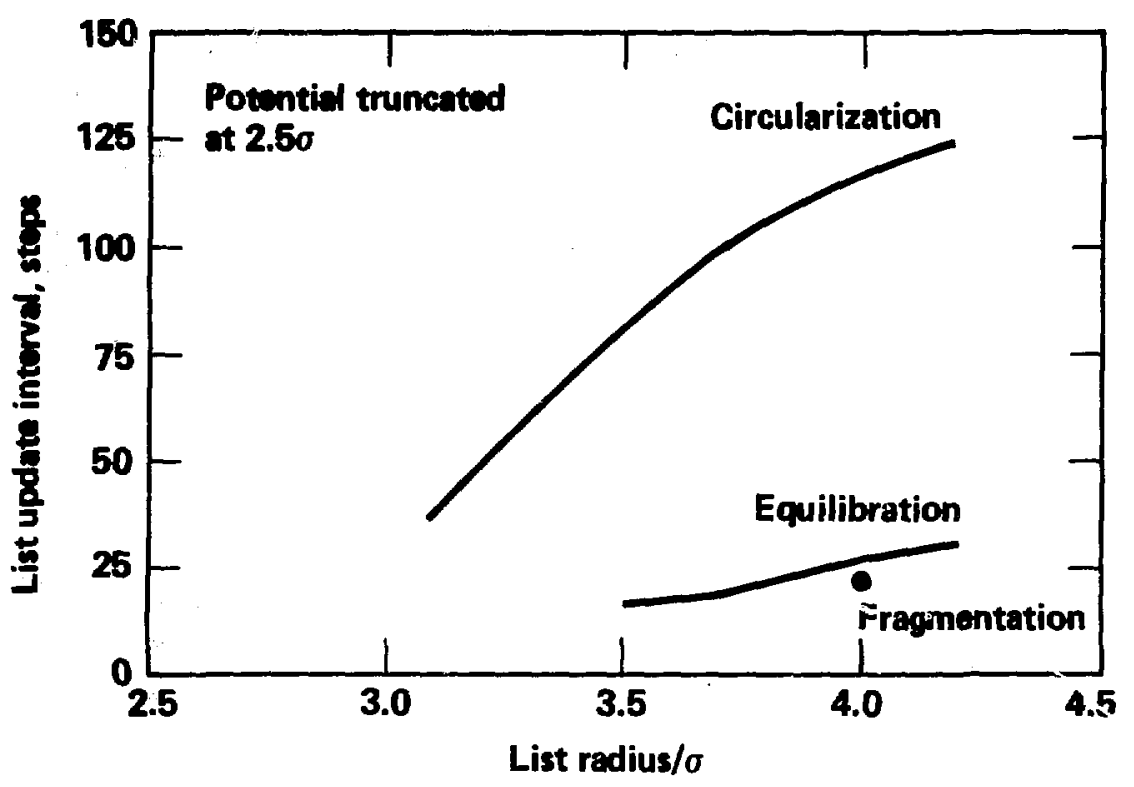


Fig. 6.23. List size for a 2611 atom system.

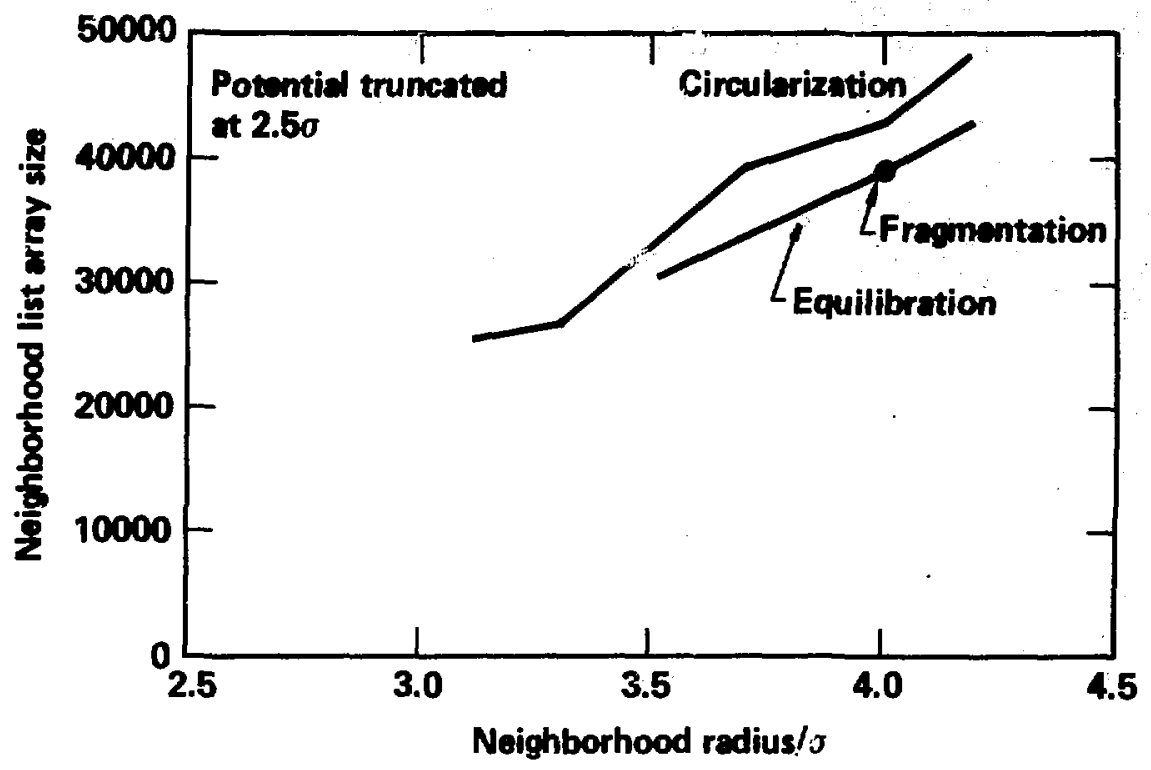


Table 6.2. Computational efficiency measures during fragmentation runs on various sized systems.

\begin{tabular}{|c|c|c|c|}
\hline $\begin{array}{l}\text { Size } \\
\text { (ntome) }\end{array}$ & $\begin{array}{c}\text { TIme Per Step } \\
\text { (etes) }\end{array}$ & $\begin{array}{l}\text { List Update } \\
\text { Interval (steps) }\end{array}$ & $\begin{array}{l}\text { List } \\
\text { Size }\end{array}$ \\
\hline 169 & 0.0067 & 25 & 2,170 \\
\hline 721 & 0.042 & 18 & 9,870 \\
\hline 2611 & 0.35 & 18 & 37,700 \\
\hline 14491 & 6.3 & 21 & 212,000 \\
\hline
\end{tabular}


Chapter 7: Two-Dinensional Molecular Dynemic Experiment Results

In this chapter, the molecular dynamics results are presented and analyced In Lerms of the four models derive 1 in Chapter Five. First, the input parameters required by the models are determined. The relationship between the quantity of isochori- heat added and the temperature rise is calculated. (The temperature rise, $\Delta T$, is en independent varieble in the some of the plots of the results.) The material properties (Grineisen parmeter, mave smed, and surface tension) are estimatsd. These four input functlons are incorporated into the models, and predictions are generated for the fragment slze and translational temperature. The molecular dynanics results are discussed and then compared to the predictions. Finally, the effect of the zero vapor pressure initial condition is estimated. 
7.1. Partition of Isochoric Heating Energy Between Kinetic and Potential Energies

A serles of isothermal molecular dynamics calculations was run at temporatures renging from saturation $(-0.5)$ to 1.2 for $169,72.1$, 2611 and 14491 atom systems confined by a repulsive wall to $P_{\text {avg }}$ 0.6, value roughly midway between the critical and liquid triple point densities. The calculations began with the wall-confined $T$ 0.5 configuration, and velocity adjustments at each time step mintalned the desired temperature increase. The potential energy Increased with time and then osclllated; the change from increase to oscillation occurred by the first energy compilation ( -1.5 ps) in each case. Because this "energy equilibration time" is both size Independent and much less than the wave traversal time in an ICF reactor liquid region, the molecular dynemics fragmentation runs should begin with an equilibrated configuration. The equllibration calculations were for times ranging between 25 and 80 reduced time units ( 4 to 12 ps) with six to ten intermediate energy compilations.

The results are summarized in Fig. 7.1 which shows the increases in kinetic energy and potential energy per atom as a function of temperature increase. The values are for an average of the energy values at the compllation times. Time averaging is required since the energles fluctuate ing atomic motion; however, the number of times used in these averages is not adequate for high precision. If the calculations were repeated, energies would be calculated at nore intermediate times to improve the average. The curve labeled "fit" is described by the equation

$$
\Delta E / N \equiv E=1.45(\Delta T)^{0.847} \text {. }
$$


Fig. 7.1. Increase in potential and kinetic energies in the $p=0.6$ molecular dynanics runs after the temperature is increased and mintained at a higher value. The energles are normallized to zero at saturation $(T=0.53)$. Henderson's Monte Carlo data are also shom; they have been slightly edjusted to match the molecular dynenics results at $\mathrm{kT} / \mathrm{c}=1.0$.

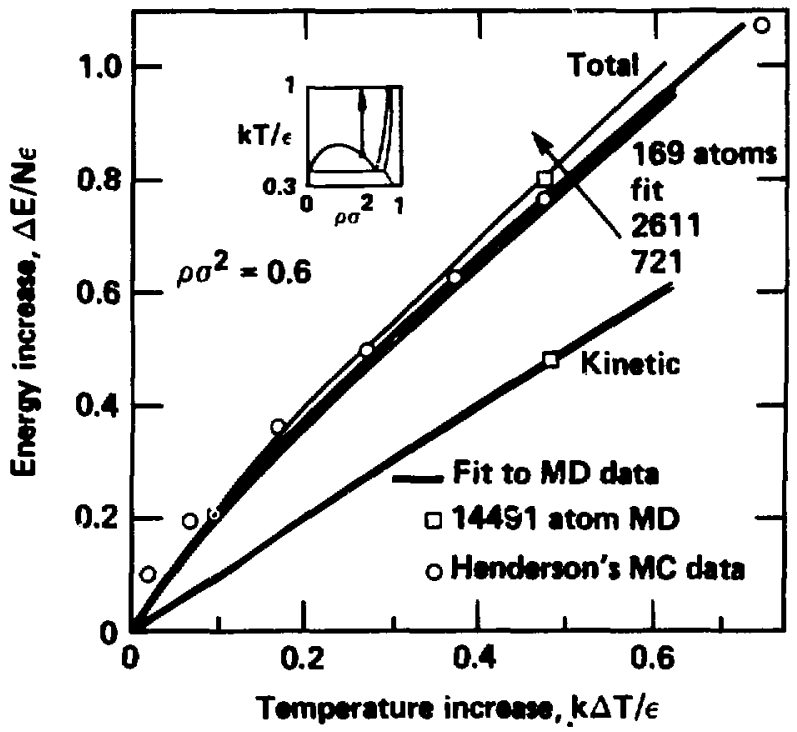


This equation has the correct limiting value of zero at zero $\Delta T$, and it fits the molecular dynamics data reasonably well.

Henderson ${ }^{24}$ reported internal energies derived from Monte Carlo calculations at $\rho=0.6$ for temperatures between 0.55 and 1.25 . When the molecular dynamics energies are adjusted to match Henderson's value at $T=1.0$, the agreement is within 4X for the lower seven temperatures. For temperature of 1.25 , the error is $7 \%$ in the opposite direction Indicating that Equation 7.1 should not be extrapolated beyond its last data point of $T=1.15$. The unadjusted values of the molecular dynamics energles also agree with Henderson's Monte Carlo data. For example, at $T=0.75$, for 2611 atoms, the conflgurational plus thermal energy per atom is -0.93 compared to --0.95 from Henderson. The higher energy in the molecular dynemics calculation is probably due to the wall which acts as an artifictal surface and Increases the interior pressure and energy. 


\subsection{Material Properties}

To calculate the Iiquid fragmentation using the Grady-Glenn nodel, three propertles are required: The Gronelsen parameter (I), wave speed (c), and surface tension ( $Y$ ). These properties are calculated below for the two-dimensional Lernard-Jonesium material using the literature and using molecular dynemics as a calculational tool. 


\subsubsection{The Groneisen Parameter}

Three methods were used to determine the Grüneisen paraneter,

$r$ : analytlc theory for gases and solids, calculation from published 11quid temperature-density-pressure data, and calculation from a molecular dynemics simulation.

For a gas,

$$
P=\text { okT }
$$

and

$$
\frac{E}{N}=\frac{D}{2} k T
$$

Where $D$ is the number of dimensions. By definition,

$$
\Gamma=\frac{1}{p}\left[\frac{\partial P}{\partial(E / N)}\right]_{V}
$$

These equations can be comblned

$$
\begin{aligned}
\Gamma & =\frac{1}{\rho}\left[\frac{\partial(\rho k T)}{\partial(\mathrm{DkT} / 2)}\right]_{V} \\
& =\frac{1}{\rho}\left[\frac{\Delta k \Delta T}{\mathrm{DK} \Delta T / 2}\right]=\frac{2}{D}
\end{aligned}
$$

Hence, for a gas, the Grüneisen parameter is 1.0 in 20 and 0.67 in 30 . For solld, the analytlc theory begins with a result from Slater, 56

$$
r_{1}=-\left[\frac{\partial\left(\ln v_{1}\right)}{\partial(\ln v)}\right]_{T}
$$

The logarithmic derivative is independent of multiplicative factors, allowing replacement of the frequencies, $v_{1}$, by angular frequencies, $w_{1}$, where 1 specifies the vibration mode. Also, 
$\partial / \partial(\ln V)=-\partial / \partial(\ln p)$. If all the modes heve equal

frequencies, then

$$
\Gamma=\left[\frac{\partial(\ln \omega)}{\partial(\ln \rho)}\right]_{T}
$$

For harmonic vibration, $\omega=(k / m)^{1 / 2}$, and $k=a^{2} \phi / \partial r^{2}$. If the repulsive part of the potential is proportional to $I^{-n}, k$ is proportional to $r^{-(n+2)}$. The density, $\rho$, is proportional to $r^{-D}$ where $D$ is the number of dimensions; thus

$$
\begin{aligned}
\omega & \sim k^{1 / 2} \sim\left[r^{-(n+2)}\right]^{1 / 2} \\
& \sim\left[\rho^{-1 / D}\right]^{-(n+2) / 2} \sim \rho^{(n+2) / 2 D}
\end{aligned}
$$

And

$$
\Gamma=\left[\frac{\partial(\ln w)}{\partial(\ln \rho)}\right]_{T}=\frac{n+2}{20}
$$

For an $r^{-12}$ repulsive potential, $r$ is 3.5 and 2.3 for two and three dimensional solids, respectively.

The published Lennard-Jonesium liquid temperature-density-pressure equation from Barker ${ }^{22}$ was discussed in Section 4.2. The equation is cross piotted at $p=0.6$ in Fig. 7.2 which also shows Henderson's Monte Carlo data (Section 4.2) and the molecular dynamics pressure data computed from the isothermal equilibration runs described in Section 7.1. Berker's cross-plotted equation and Henderson's data were fit to quadratic forms (see Table 4.5).

The molecular dynemics pressures include values computed from the radial repulsive forces between the atoms and the wall and, in three cases, PV data (using Eq. 6.36). In most cases, the pressures were 
F1g. 7.2. Pressures from the molecular dynamics runs, Barker's theoretical and Monte Carlo calculations ${ }^{22}$, and Henderson's Monte Carlo calculations 24 .

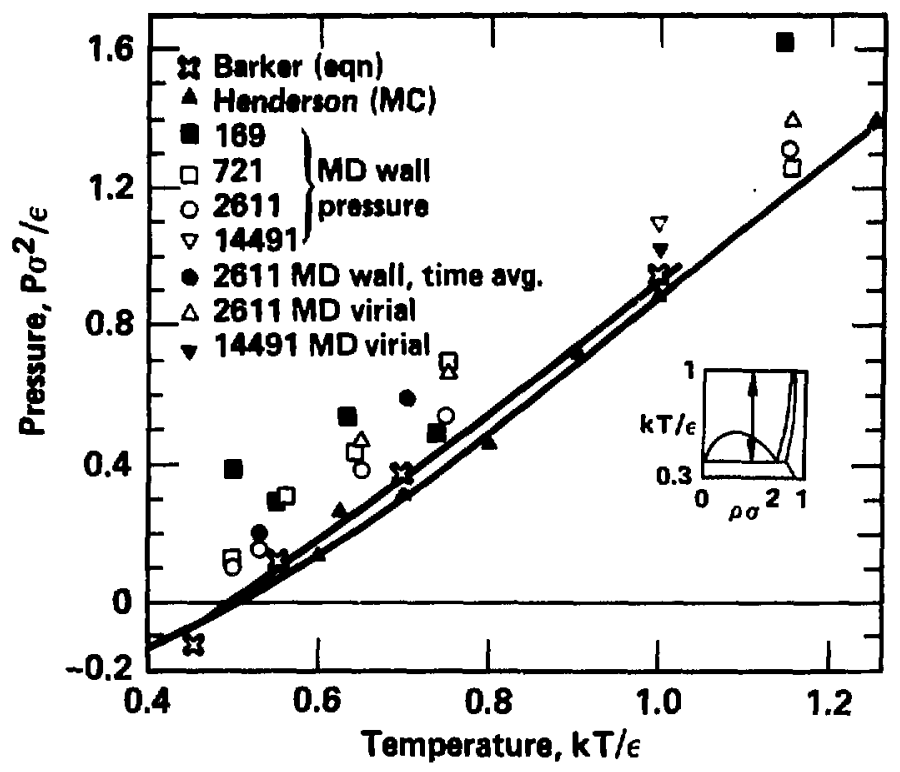


time averaged from only six to ten widely separated insteneneous values; therefore, they are imprecise. (In two cases, the data were averaged over $\sim 185$ time steps.) However, all the nolecular dynamics data lie above the Monte Carlo data. This trend is probebly due to the molecular dynemics boundary conditions. The repulsive wall represents an artificial surface which increases the internal liquid pressure to a value higher than for the theoretlcal and Monte Carlo calculations; however, the wall is required to confine the systen during the time averaging perlod. The alternative, perlodic boundary conditions, does not allow circular surfaces. Also, since the wall is purely repulsive, there is no opportunity for mutual cancellation of negative and positive forces, as would be observed by a test surface within the bulk Ilquid.

The Grüneisen parameter is

$$
\Gamma=\frac{1}{\rho}\left(\frac{\partial P}{\partial E}\right)_{Y}=\frac{I}{\rho}\left(\frac{\partial P / \partial T}{\partial E / \partial T}\right)_{V}
$$

The pressure derivative is available from both Barker's pressure equation and Henderson's data (see Table 4.5). The energy derlvative, $c_{V} \equiv(\partial E / \partial T)_{V}$ is also available from Henderson's data (Table 4.5 and Fig. 4.11). These derivatives were used to plot the Groneisen parameter as a function of the temperature for three densities (fig. 7.3). This figure also shows the solid and gas results and the known Graneisen paraneters of liquid lithium and lead.

The liquid results should eventually decline to the ideal gas value at high temperature. The lower density $(0.5$ and 0.6$)$ results are doninated by the specific heat (วP/JT is roughly constant). However, the high density $(0.7)$ result is dominated by the pressure 
F1g. 7.3. Groneisen parameter from the Monte Carlo data of Henderson and Barker. Solid, gas and liquid netal results are also presented. The values plotted include the factor $(0 / 2)$ where $D$ is the number of dimensions. Thus, two dimensional results can be read directly while the values should be multiplied by $2 / 3$ to obtain three dimenslonal numbers.

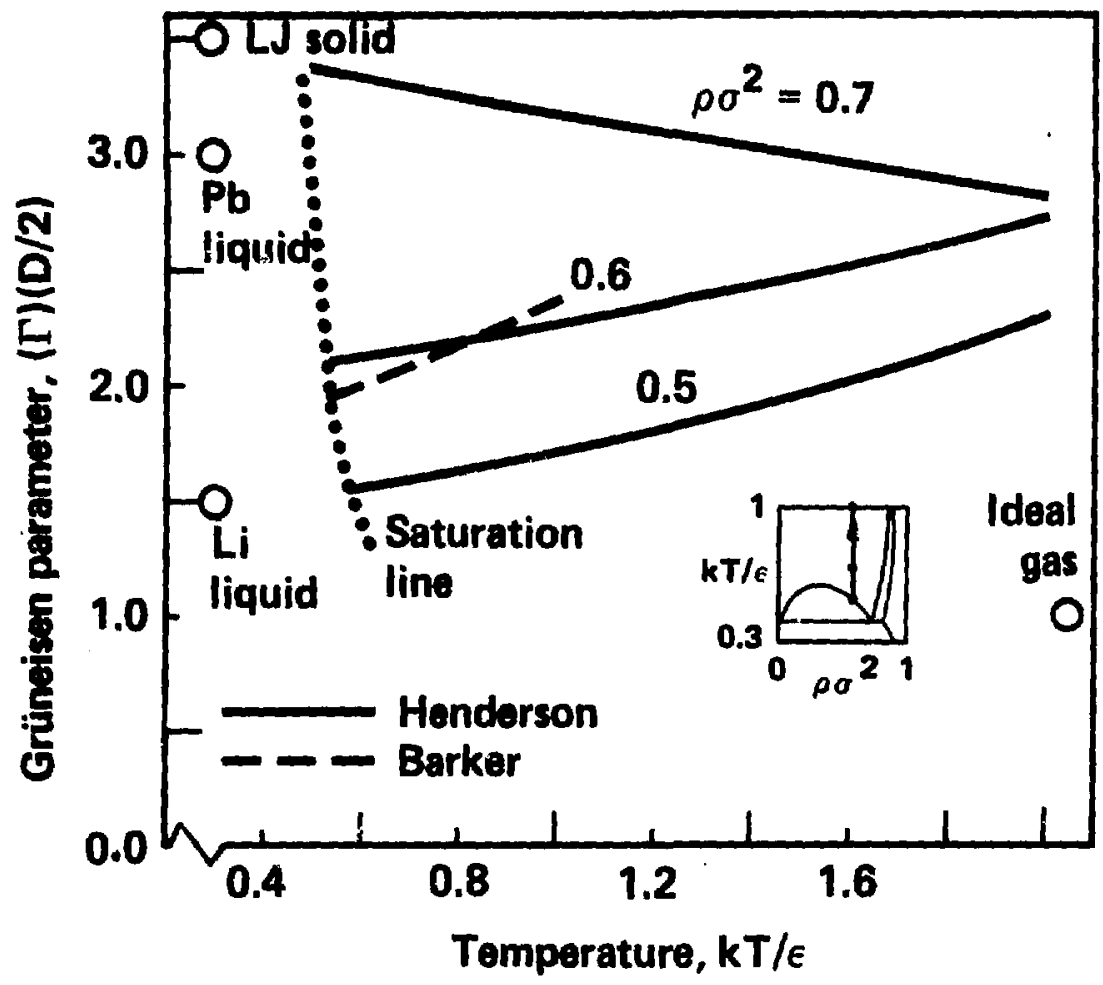


derivative ( $c_{v}$ is roughly constant). The shift in the dominant factor is responsible for the sign change in tive slope of the Grônelsen parameter.

As density increases, the liquid Groneisen parameter approaches the solid value. However, considerably higher tempecatures are apparently required before the liquid values converge to the ideal gas limit.

Since the molecular dynamics resuits are concerned with the final pressure produced, rather than the local (in temperature) value of the Grüneisen parameter, a cumulative Grüneisen paremeter was also calculated from the Monte Carlo data of Henderson ${ }^{24}$ and Barker 2.2 (F19. 7.4).

$$
\Gamma_{c}=\frac{1}{\rho}\left[\frac{P-P_{\text {sat }}}{\left[E-E_{\text {sat }}\right.}\right]
$$

The final Grünelsen calculation used two molecular dynamics calculations with energy and wall pressure compllations at, each time step $(-0.004 \mathrm{ps})$. The 1sothermal equilibration code was used with a stationary repulsive wall ard temperature adjustments on each time step. Beginning with a $T=0.53$ equilibrated liquid, 300 steps were run at both $T=0.53$ and $T=0.7$. The pressure on the wall was calculated at each time step by sumning the repulsive forces between the atoms and the wall. The higher temperature run took 110 time steps to partition the added energy into potential plus kinetic energy. After this tine ( $\sim 3$ reduced time units or 0.5 ps), both runs displayed fluctuating pressures and energies around average values (Figures 7.5 and 7.6). The pressures were averaged over the 
Fig. 7.4. Cumulative Groneisen parameter defined by the pressure and energy differences between the heated state and the saturated Ilquid at the same density. This plot also shows the single cumulative Gronelsen parmeter calculated from the two molecular dynamics runs.

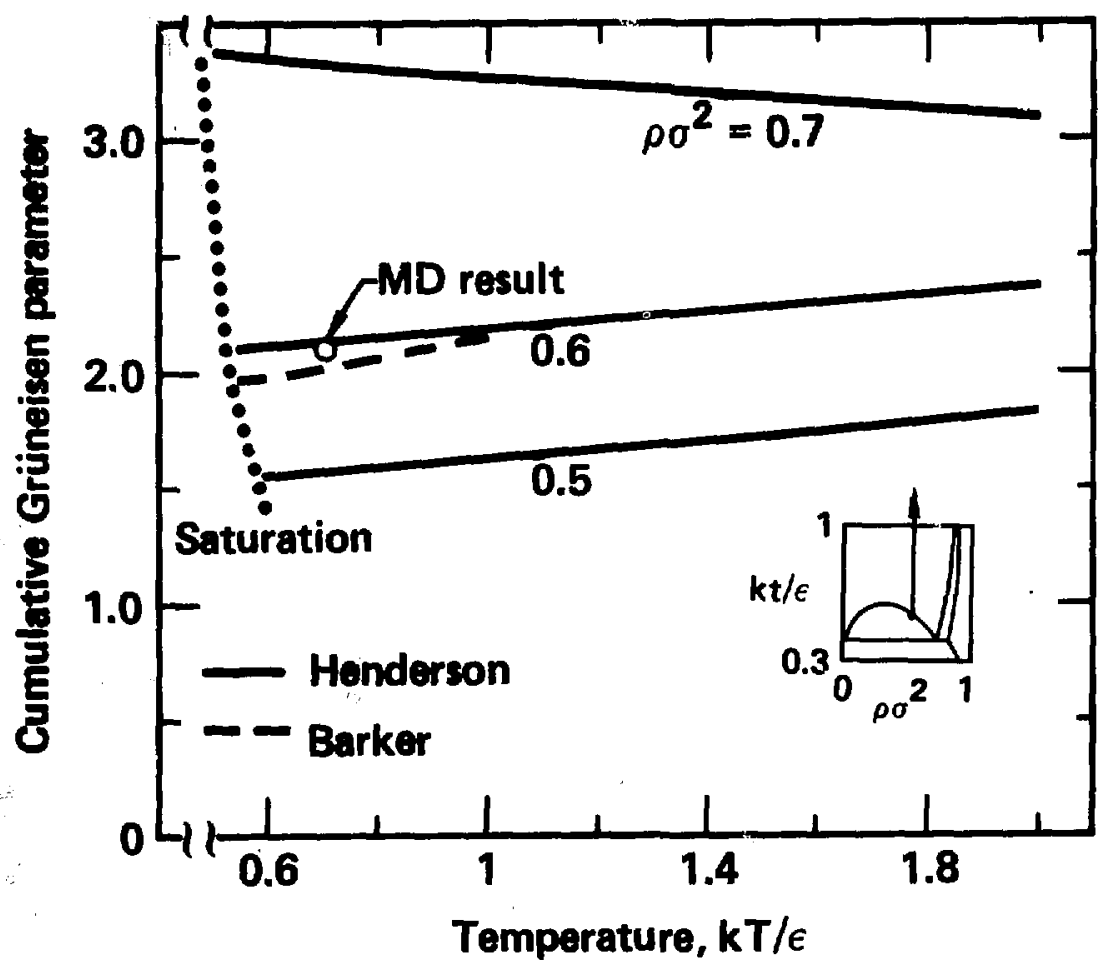


F1g. 7.5. Pressure as a function of time for two constant volume and temperature molecular dynamics calculations.

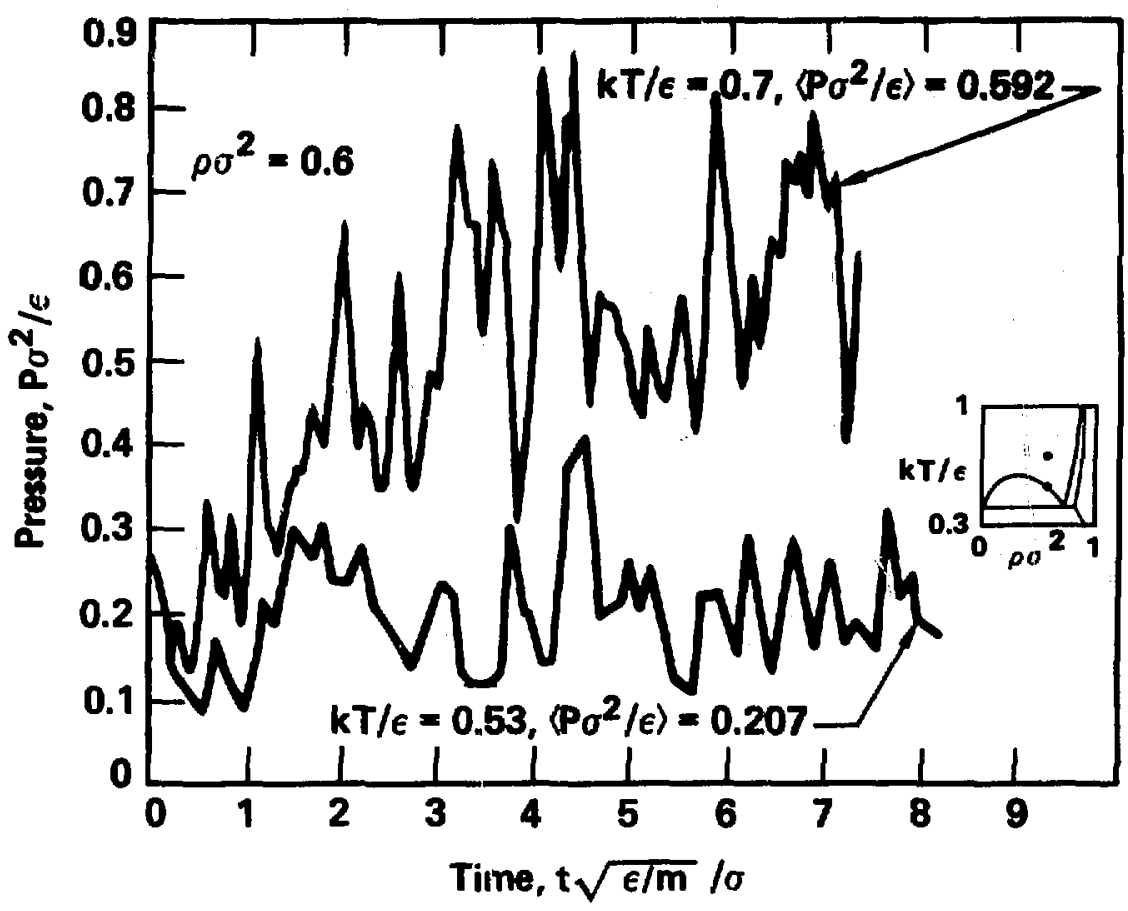


Fig. 7.6. Configurational plus kinetic energy per atom for two 2611 atom liquids confined by walls. The density was 0.6 for both the $T=0.7$ and $T=0.53$ (saturated) cases. The energy zero is at rero temperature with all first nelghbor bonds at a minimum potential $(-c)$.

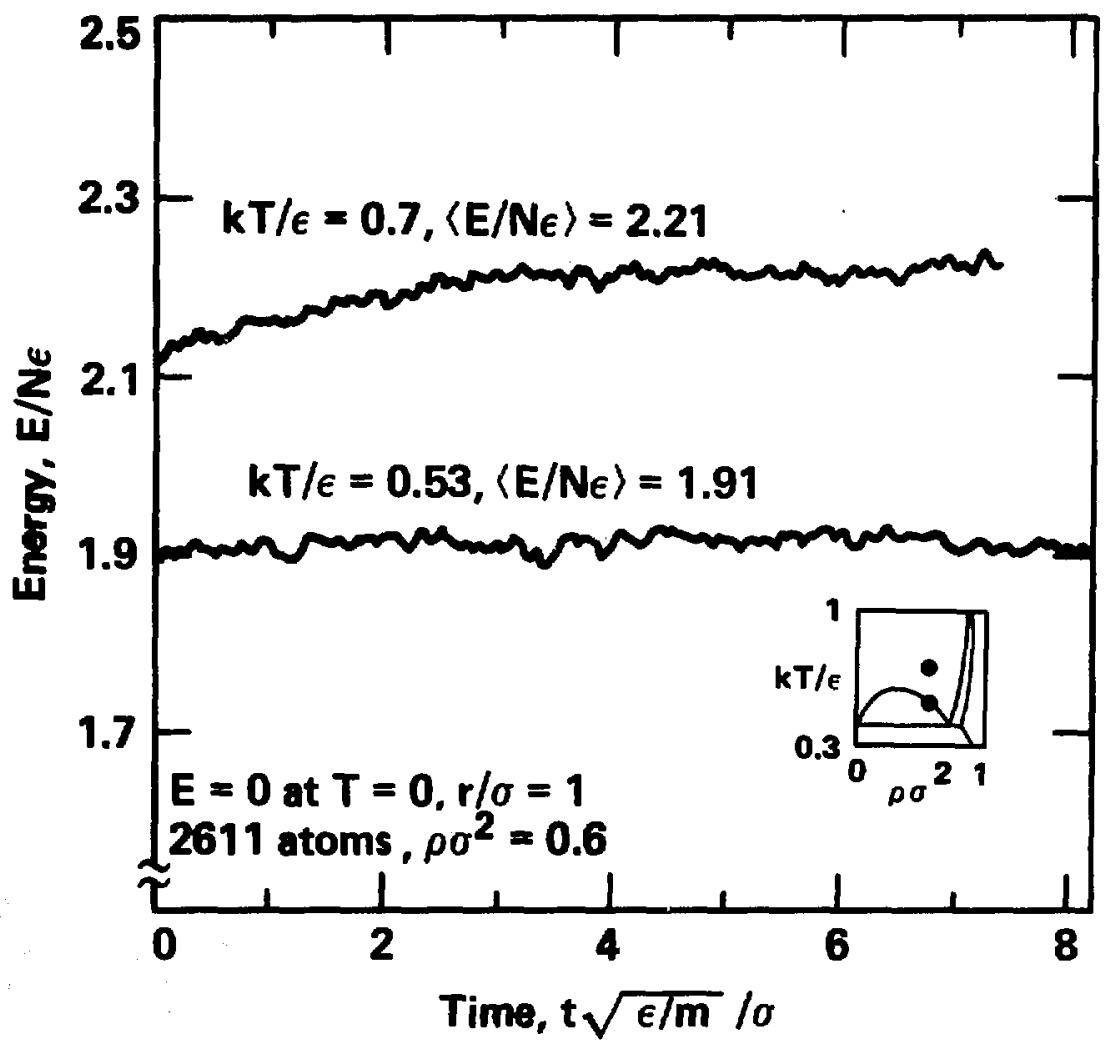


last -5 tine units and values of 0.592 and 0.207 wre obtalned for the $T=0.7$ and 0.53 runs, respectively. The time avereged energy increase was 0.3 reduced units ( 1.76 AT), about ox lowr then the relatively crucie prediction of Fig. 7.1. The Grtheisen paremeter is then,

$$
r_{c}=\frac{1}{p} \frac{\Delta P}{0.6}\left(\frac{1}{0.592-0,207}\right)=2.1 \quad .
$$

Th1s result agrees with the results calculated from Barker's and Henderson's data us shown in F10. 7.4. The Hendsrson pressure (end derived Grönelsen parmater) data were used in the comparison of the molecular dynemics fragnentation data with the four hydrodynamic models from Chapter Five (Section 7.4). 


\subsubsection{Wave Speed}

The wave speed was evaluated at the heated temperature since that is the temperature at wich the wave travels. Two approaches to calculating the wave speed were taken. First, the Henderson ${ }^{24}$ and Barker 22 pressure data (Section 4.2) were used to compute the sound speed. Then, the molecular dynamics runs were used to estimate the wave conversence time, $T=R / C$.

The soind speed is

$$
c=\left[\left(\frac{\partial P}{\partial p}\right)_{S}\right]^{1 / 2}
$$

Thermodynamic relationships can be used to convert equation 7.13 to more measurabie quantities

$$
\left(\frac{\partial P}{\partial \rho}\right)_{S}=\frac{C_{P}}{C_{V}}\left(\frac{\partial P}{\partial \rho}\right)_{T}
$$

where $c_{p}$ and $c_{v}$ are the specific heats at constant pressure and volume, respectively.

$$
c_{p}=c_{V}+T V a_{v}^{2} a_{T}
$$

where $q$ is the volumetric coefficient of thermal expansion, (OV/OT $)_{P} / V$, and $B_{T}$ is the isothermal bulk modulus, $-V(a P / \partial V)_{T}$. The coefficient of expansion can be eliminated by using

$$
\left(\frac{\partial P}{\partial V}\right)_{T}\left(\frac{\partial Y}{\partial T}\right)_{P}\left(\frac{\partial T}{\partial P}\right)_{V}=-1
$$

and $\partial / \partial V=-\rho^{2} \partial / \partial \rho$ can be used to eliminate the volume. Finally, 


$$
c_{v}=\left(\frac{\partial F}{\partial T}\right)_{V}
$$

Then,

$$
c=\left\{\left(\frac{\partial P}{\partial \rho}\right)_{T}\left(1+\left[T\left(\frac{\partial P}{\partial T}\right)_{V}^{2}\right] /\left[p^{2}\left(\frac{\partial P}{\partial \rho}\right)_{T}\left(\frac{\partial E}{\partial T}\right)_{V}\right]\right)\right\}^{1 / 2} .
$$

Equation 7.18 was evaluated at temperatures of $0.55,0.7$, and 1.0 using Barker's 22 pressure equation (Eq. 4.17 and F1g. 4.9) and Its crossplot (FIg. 4.10 and Table 4.5) In confunction with speclfic heats derived from Henderson's ${ }^{24}$ Monte Carlo data (FIg. 4.11 and Table 4.5). However, intermediate temperatures could not be directly evaluated using Barker's equation since the constants in Eq. 4.17 ( $f_{1}$ through $g_{4}$ ) are specified only at four temperatures. Henderson's Monte Carlo data were also used to evaluate the sound speed (Eq. 7.18) at a range of pressures and temperatures (using the analytic fits summerized in Table 4.5). The results are shown in Flg. 7.7, and the values of the varlous terms in Eq. 7.18 are listed In Table 7.1 for the three points using Barker's pressure data. The agreenent in the sound speeds calculated from the two references is sat1sfactory.

The molecular dynamics runs were examined every thirty time steps following the removal of the confining wall. Plots of the density and cumulative density,

$$
\sum_{0}^{T}(\rho(x) \Delta V) / \sum_{0}^{T} \Delta V
$$

(and in some cases, the cumulative radial velocity), as functions of radial position were generated at each tine. In two cases, a movie of 
F1g. 7.7. Sound speed computed from Henderson's 24 Monte Carlo pressure and energy data and from Barker's 22 pressure equation.

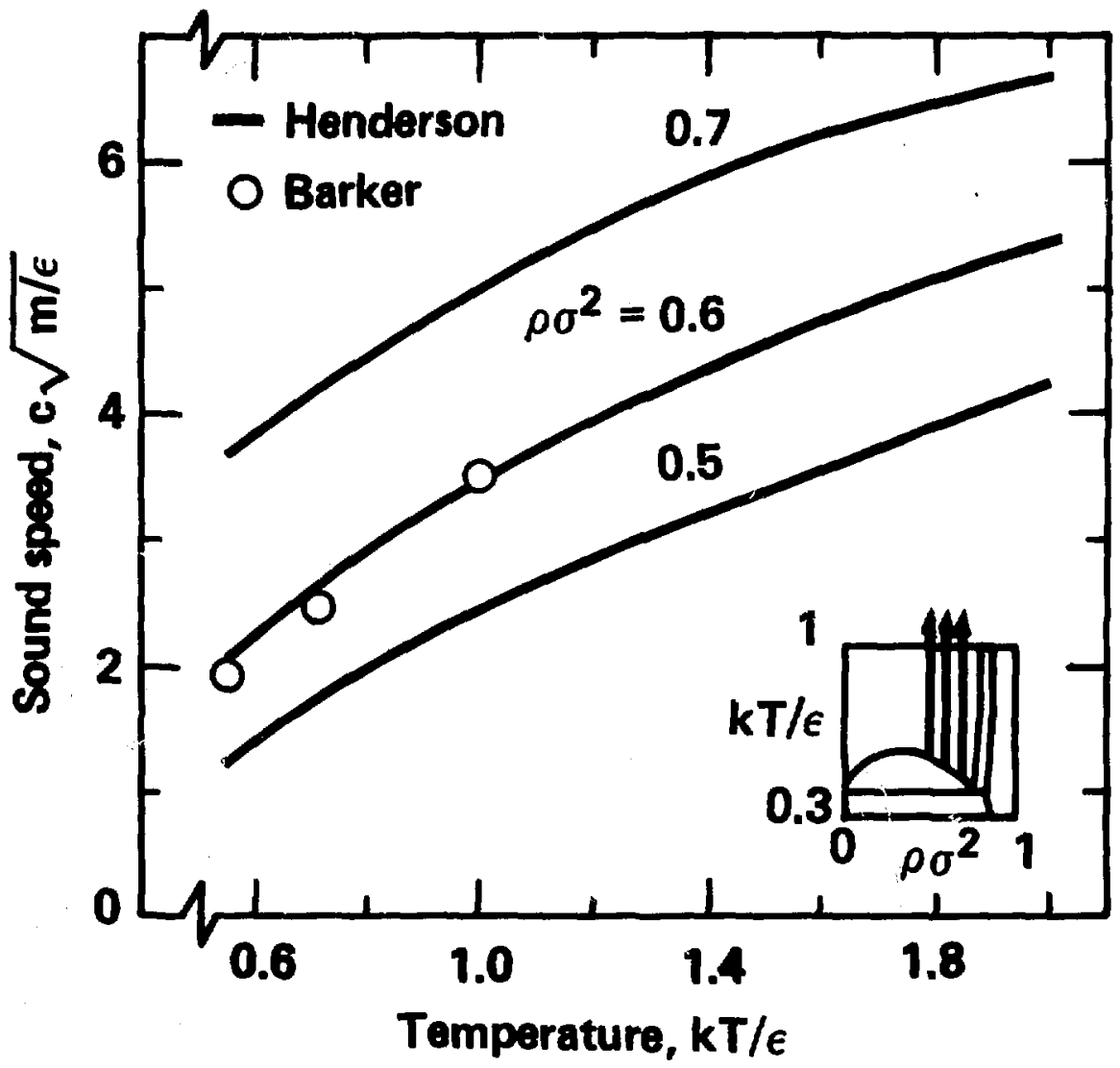


Table 7.1. Calculation of the sound speed (Eq. 7.18) for a constant density of 0.6 and temperatures of $0.55,0.7$ and 1.0. Source codes are "H" for Henderson and "B" for Barker. Reduced varlables are used $\left(T=k T / c, \rho=\rho \sigma^{2}, P=P \sigma^{2} / c\right.$, and $c=c$ rw/ $)$.

Temperature

\begin{tabular}{l|l|lll} 
& & 0.55 & 0.7 & 1.0 \\
\hline$P$ & $B$ & 0.132 & 0.356 & 0.936 \\
\hline$(\partial P / \partial T)_{V}$ & $B$ & 1.77 & 1.84 & 1.97 \\
\hline$E-E_{0.53}$ & $H$ & 0.030 & 0.252 & 0.660 \\
\hline$(\partial E / \partial T)_{V}$ & $H$ & 1.57 & 1.55 & 1.51 \\
\hline$(\partial P / \partial \rho)_{T}$ & $B$ & 0.55 & 2.24 & 5.04 \\
\hline$\left(\frac{\partial P}{\partial \rho}\right)_{T}^{1 / 2}$ & & & & \\
\hline$C$ & & 0.74 & 1.50 & 2.24 \\
\hline$c /\left(\frac{\partial P}{\partial \rho}\right)_{T}^{1 / 2}$ & & 1.90 & 2.55 & 3.49 \\
\hline
\end{tabular}


the atomic positions was produced. A typical density result is shown in F1g. 7.8 .

For most of the molecular dynamics runs, the time that the rellef wave reached the center can be estimated to be between two compllation times. Although the runs were intended to begin at exactly $\rho=0.6$, the actual densities varied between 0.61 and 0.73 . The wave traversal tIine (R/c) date were adjusted to remove the Influence of the density. The adjustment was derived from the variation of the prevlously generated sound speed curves with density (FIg. 7.7). The ratlo of the $(\rho=0.7)$ curve to the $(\rho=0.6)$ curve is almost identical to the ratio of the $(p=0.6)$ curve to the $(p=0.5)$ curve. A fourth order equation,

$$
F_{p}=3.4862-5.7303 T+5.9473 T^{2}-2.7619 T^{3}+0.4712 T^{4}
$$

is mloway between the two ratlo eurves. This equation was used to produce a normalized sound speed,

$$
c^{*}= \begin{cases}c /\left(1-\rho p^{*}+p_{p}^{*} F_{p}\right) & , p 0.6 \\ c /\left(1-\rho p^{*}+F_{p}\right) & , p 0.6\end{cases}
$$

where

$$
\rho^{*}= \begin{cases}(0.6-p) / 0.1 & , p 0.6 \\ (p-0.6) / 0.1 & , p 0.6\end{cases}
$$

The results of density-normalizing the sound speed curves and the molecular dynamics data are indicated in Fig. 7.9. The convergence of the sound speed curves justifies the normalization, at least for 0.5 p 0.7. The vertical renge for each molecular dynamics run indicates the difficulty in reading the have traversal time from the 
Fig. 7.8. Density and cumulative density profiles 330 steps (time = 9.06 reduced units or $1.4 \mathrm{ps)}$ ) aftor removal of the wall from a liquid equilibrated at $\mathrm{kT} / \mathrm{c}=\mathbf{0 . 6 5}$. The initial cumlative density profile is also shown. Comparison of the two cumulative density profiles indicates the tensile relief wave has penetrated to $r \sim 15$ reduced units $(4 \mathrm{~mm})$.

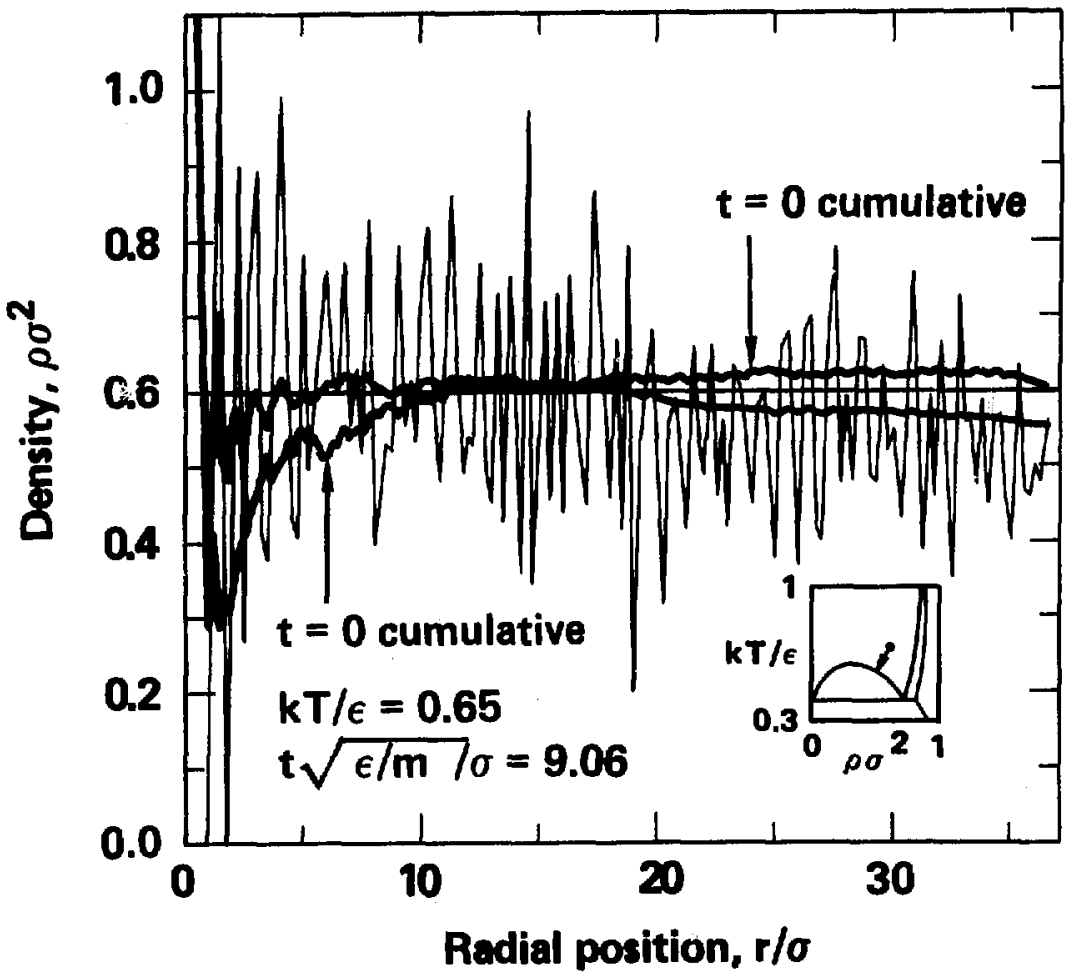


F1g. 7.9. Average wave speed for each of the molecular dynamics Iuns. The sound speed curves from Fig. 7.7 are repeated. All speeds have been adjusted to the value expected at $\rho \sigma^{2}=0.6$. The variation in the curves shows the accuracy of the adjustment. The vertical ranges in the nolecular dynemics results are due to the difficulty In separating the wave motion from the background thermal notion.

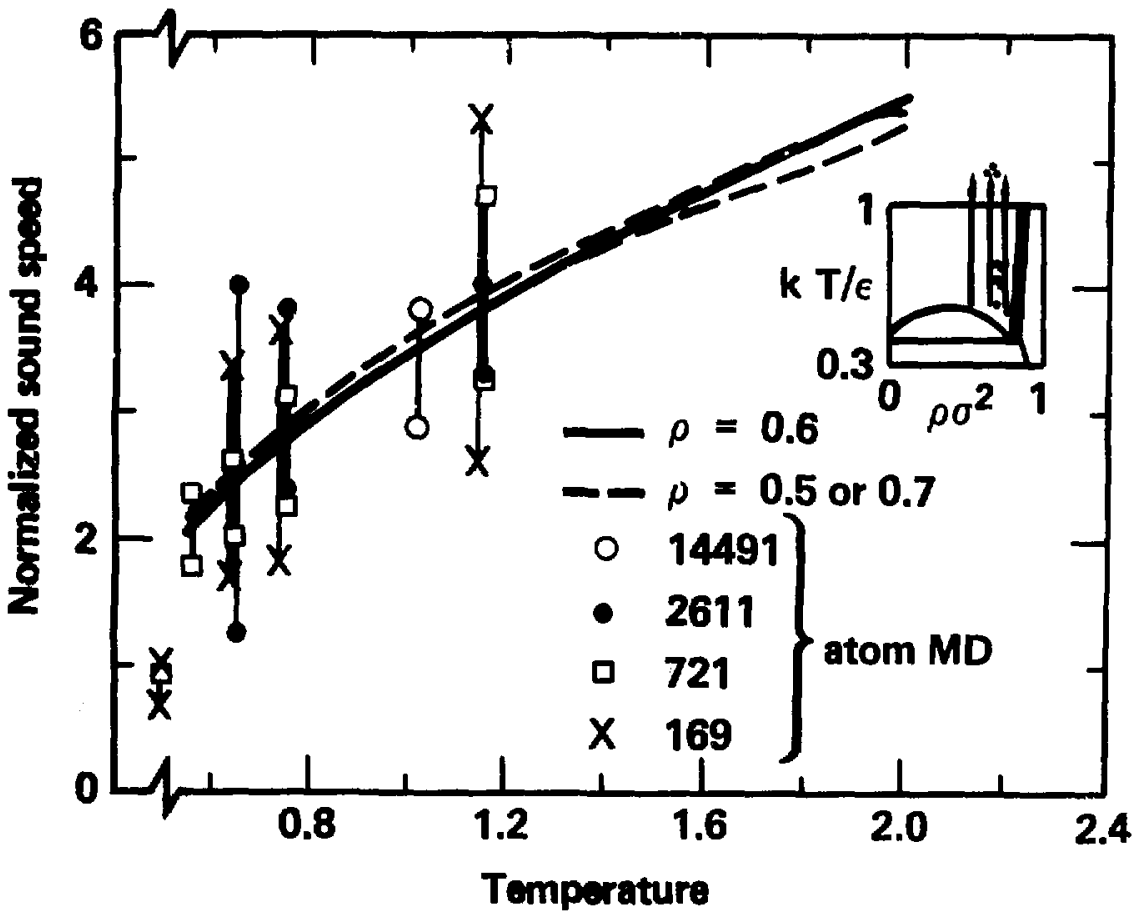


molecular dynamics results.

Although the molecular dynamics results do not disagree with the sound speed curves, they also do not confirm them. Further, in some cases, the molecular dynamics data could be interpreted as indicating an acceleration in the rarefaction wave as it converged.

To Investigate the unexpected possibility of an accelerating rarefaction wave, two additional sets of calculations were carried out. The first set began with an $\mathbf{8 2 3}$ aton clrcular crystal at zero temperature. Densitles of 1.01 and 0.6 were used, producing a strong expansion wave and a somewhat weaker compression wave. The results are shown in Fig. 7.10. The position tracks indicate the location where the particle velocity increases to $10^{-1}$ reduced units as a function of time. These tracks are essentially straight except when the wave slows as it approaches the center (where no new atoms are avallable to the wave). The two cumulative velocity anplitude curves have maxima at about the times that the wave reaches the center. At later tines, the outer regions reverse and the crystal motion begins to rebound; this behavior is due to the abllity of the material to sustain tensions of this level without fracturing.

The second set of calculations used the AFTON hydrodynamics code ${ }^{18}$. Two compressed materlals were released in both slab and cylindrical geometries. For both the perfect gas and the Lennard-Jones liquid, the expansion wave had Identical tracks on the radius-time graph for both geometries, Indicating that the ability of the Lennard-Jones Ilquid to sustain tension has no impact on its wave speed in a converging geonetry. 
Fig. 7.10. Wave front tracks and particle velocity magnitudes for converging expansion and compression wäves.

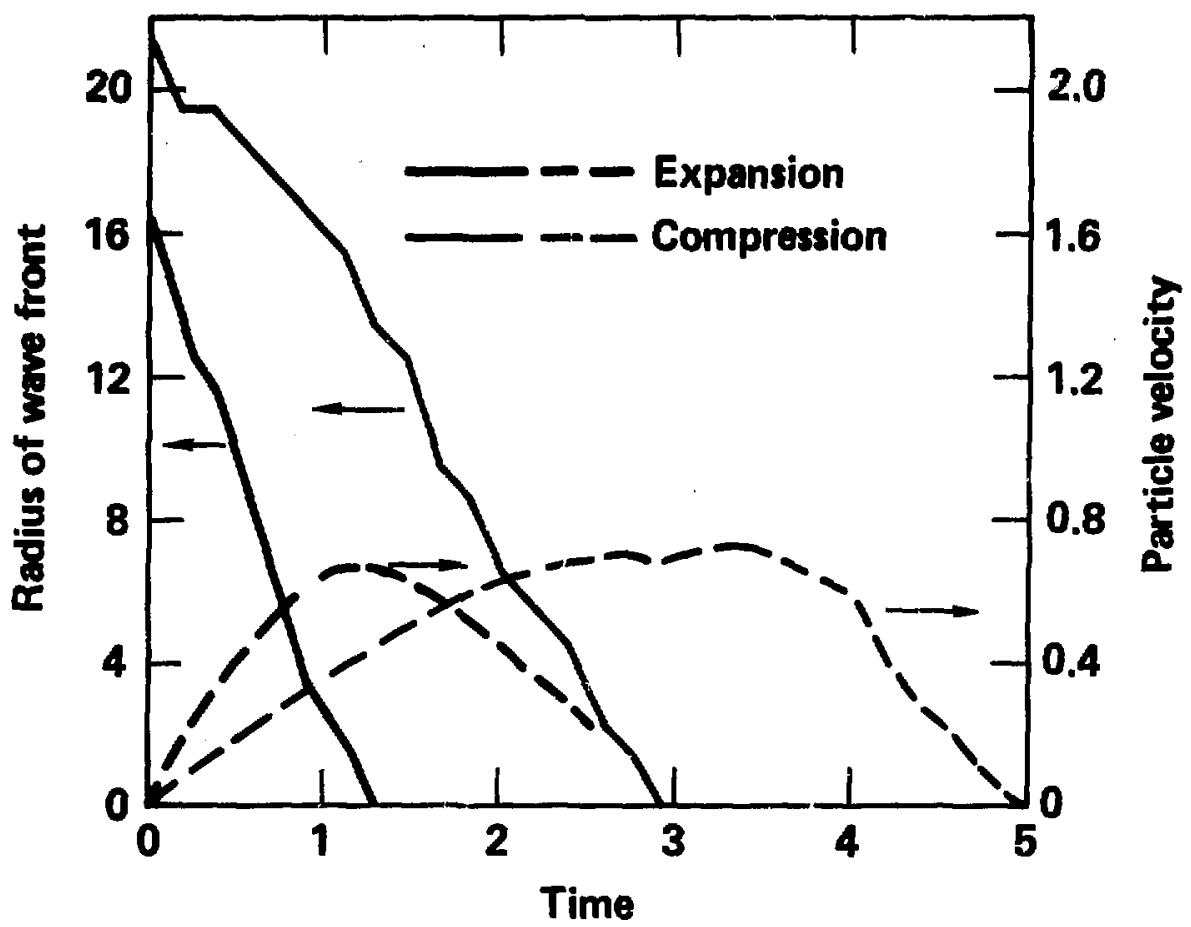


These two experiments (nolecular dynemics and hydrodynamics code) verify that converging rarefaction waves do not acceiorate. Thus, the sound speeds read from the molecular dynamics fragmentation runs must be regarded as inexact due to the obscursing thermal notion. However, even these Inexact results do not disagree with the equation-of-state derivent sound speeds. The sound speeds darived from Henderson's date are used as data in the comparisen of the nolecular dynanics results to the four hydrodvanmig nodels from Chapter Five (Sectlon 7.4). The sensitivity to factor of two change in tire sound speed is also evaluated. 


\subsubsection{Surface Tension}

Little effort has been mode to calculate the two-dinensional surface tension for the Lennard-Jones potential. Two authors have porformod monte Carlo or molecular dynenics calculations with periodic boundary conditions to simulate two-dimensional Lennard-Jones liquids with free surfaces 23,57 . However, mither author calculated the Information required to specify the surface tension. Further, Croxton's $s^{57}$ molecular dymenics sinulation did not adsquately isolate the surface from the boundary 58 .

In three-dinensions, mole work has been published. Falls ${ }^{59}$ used a series of simplifying approximations to develop a theory capable of predicting equilibriun density and pressure profiles in small drcplets. The theory predicts that the surface tension is lower than for: plenar interface when the droplet radius is less than about ten molecular radil. Falls also offers a gond intuitive explanation for this phenomenon. Consider two cones with vertices on an atom that is nas, but not on, the drop surface (Fig. 7.11). One cone (the normal cone) opens outwerd across the droplet Interface wille the other (the tengential cono) is parallel to the surface. For a planar interface, as wll as for large and small drops, the normal cone contains only a fow atoms bacause of the surface. For a plenar interface or for a large drop, the tengential cone contains many more particles. This asymmetry produces unequal normal and tangential forces (and pressures) on the reference atom. These unequal pressures can be ascribied as surface tension. For smell drops, the tengential cone intereacti the surface, and the normal and tangentlal forces become wowe Isetropic, reducing the surfece tension. 
Fig. 7.11. Cones of particles interacting with e roforence atom for a planar interface, a large drop, and a small drop.

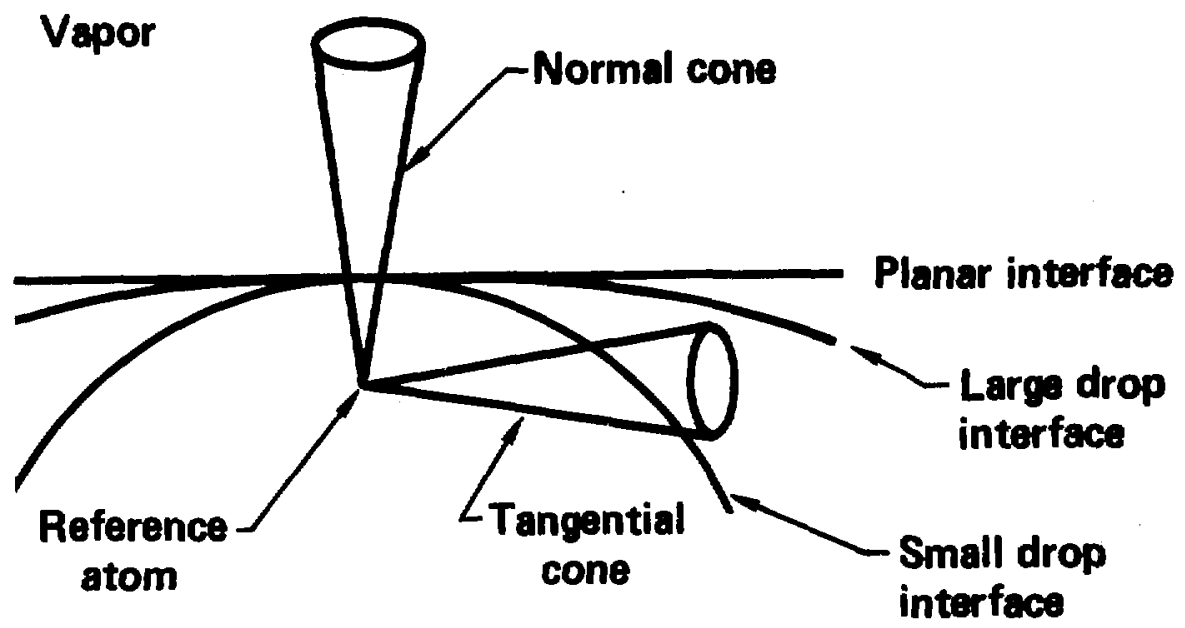

Liquid 
Brodskaya 38,52 used nolecular dynamics to compute the density and pressure profiles in small three dimensional Lennard-Jonesium drops. The calculations used time averages over about 20 reduced time units ( $t \sqrt{E / m} / \sigma)$. Recently, Thompson ${ }^{29}$ published an extensive nolecular dynemics study of surface tension that used time averages of up to 1400 reduced time units.

The results of the three studies (Falls, Brodskaya, and Thompson) are compared in Fig. 7.12. The qualitative results are similar: decreasing surface tension with decreasing drop slze and with increasing temperature $(r=0$ at the critical point and for isolated atoms). However, the quantitative results are only approximately similar. Because of the extraordinary care and long time averages taken by Thompson, that study ${ }^{29}$ is probably more accurate than the earlier Soviet studies 38,52 . Thompson ${ }^{29}$ discussed the approximations inherent in Falls' theory ${ }^{59}$ and concluded they are responsible for some "unphysical" behavior in Falls' results $\left(P_{T}\right)$ $P_{N}$ across much of the profile for small drops, implying negative surface tension). Falls attributes this behavior to being in the unstable (spinodal) region of the phase diagram, but Thompson was able to simulate stable drops of the same size (with $P_{T}<P_{N}$ and positive surface tension).

Because of Thompson's success in calculating three-dimensional surface $\operatorname{tension}^{29}$, en effort was made here to repeat the calculations in two dimensions. A wall-confined liquid at the desired denglty and temperature was the starting point. The wall radius was sudienly increased and the system evolved lito one or more liquid depes end essociated vepor. The wall was centered on the system 
Fig. 7.12. Three-dimensional surface tension as a function of temperature and drop size. The data is plotted as a function of $\left(N_{2}\right)^{-1 / 3}$ because surface tension is expected to have radius and area dependent contributions. Straight lines are used to cornect data points to group the sinllar data.

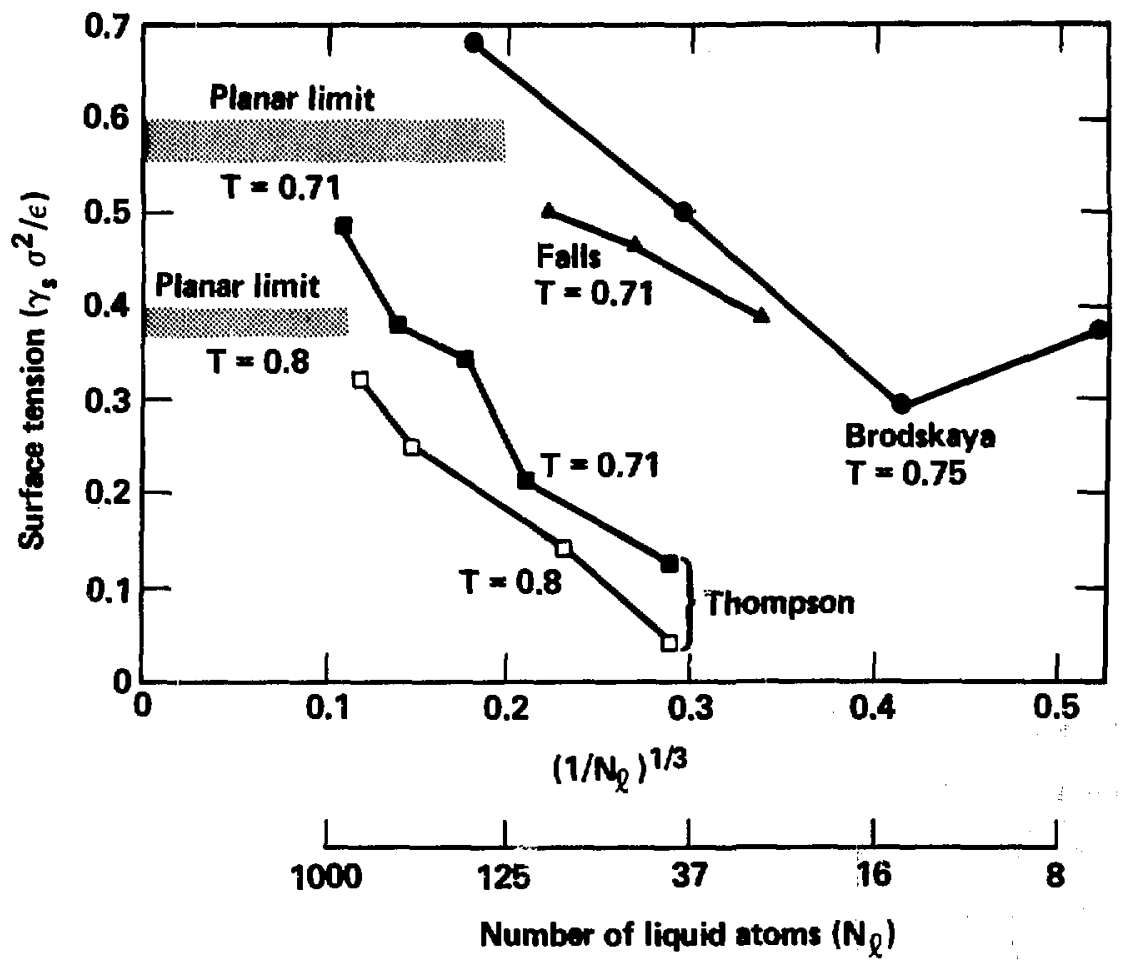


center of mass, and the velocities were adjusted for an isothermal situation to accommodate energy loss from the liquid during vapor pressure formation. Thompson 29 showed that both isothermal and constent energy calculations are valid.

If the liquid region frugmented, the velocities were adjusted to increase the imward radial kinetic eneray. The azimuthal velocities were then adjusted to preserve the overall temperature. Once the fragments coalesced, time averaging of the density and pressure proflles was reinitiated.

The surface tension calculation requires time averaged pressure and density profiles. The instantaneous density was calculated in concentric rings of width $\sigma / 4$. The instantaneous normal pressure was calculated at concentric surfaces centered in the rings.

$$
P_{N}(r)=\rho(r) k T+\frac{l}{2 \pi r} \sum_{k} F_{k}
$$

where the sum is the total of the normel components (with respect to the system center of mass) of all the interatomic bonds crossing che surface. Using a straight line force between the particles to determine the configurational pressure was originally proposed by Irving and Kirkwood ${ }^{60}$. Cholce of a different force line produces a different pressure tensor, as denonstrated by Harasima ${ }^{61}$.

As in the atomic motion calculation, two nested do-loops are used to deternine which particles have interactions. For each interaction, the minimm and maximum radll (with respect to the system center of mass) of the bond are determined, 


$$
\begin{aligned}
& r_{\max }=\max \left(r_{i}, r_{j}\right) \\
& r_{\min }=\left\{\begin{array}{l}
\frac{1}{2}\left[2\left(r_{i}^{2}+r_{j}^{2}\right)-r_{i j}^{2}-\frac{\left(r_{1}^{2}-r_{j}^{2}\right)^{2}}{r_{i j}^{2}}\right]^{1 / 2},\left|\lambda_{\min }\right| 1 \\
\min \left(r_{1}, r_{j}\right),\left|\lambda_{\min }\right| 1
\end{array}\right.
\end{aligned}
$$

where $h$ defines the vector $\vec{r}$ in terms of the position vectors of the two particles $\left(\vec{r}_{1}, \vec{r}_{j}\right)$ and the interparticle vector $\left(\vec{r}_{1 j}\right)$ as shown in Fig. 7.13.

$$
\vec{r}=\frac{1}{2}\left(\vec{r}_{1}+\vec{r}_{j}+\lambda \vec{r}_{1 j}\right)
$$

The value of $\lambda$ when $r$ is minimized, $\lambda_{\text {min }}$, is computed where $\vec{r}$ and $\vec{r}_{i j}$ or its extension meet at a right angle. Then

$$
\lambda_{\min }=\left(r_{1}^{2}-r_{j}^{2}\right) / r_{1 j}^{2}
$$

When $r_{m i n}$ does not lie between points 1 and $j,\left|\lambda_{\min }\right| 1$, and the minimum radius surface intersects the smaller radlus point.

Once the minimum and maximum redil of the bond are specified, the surfaces affected by the bond are determined. For each of these surfaces, the values of $\lambda$ at the surface-bond intersections are computed

$$
\lambda_{ \pm}=\left[\frac{r_{1}^{2}-x_{1}^{2}}{r_{1 j}^{2}}\right] \pm\left[\left(\frac{r_{1}^{2}-r_{j}^{2}}{r_{1 j}^{2}}\right)^{2}+1-2\left(\frac{r_{1}^{2}+r_{j}^{2}}{x_{1 j}^{2}}\right)+\frac{4 r^{2}}{r_{1 j}^{2}}\right]^{1 / 2} .
$$

The normal force 1 . 
F1g. 7.13. Geometry used to calculate $\lambda_{\min }$. The arcs define the radial limits within which test surfaces are affected by the bond between atons 1 and $\mathrm{J}$.
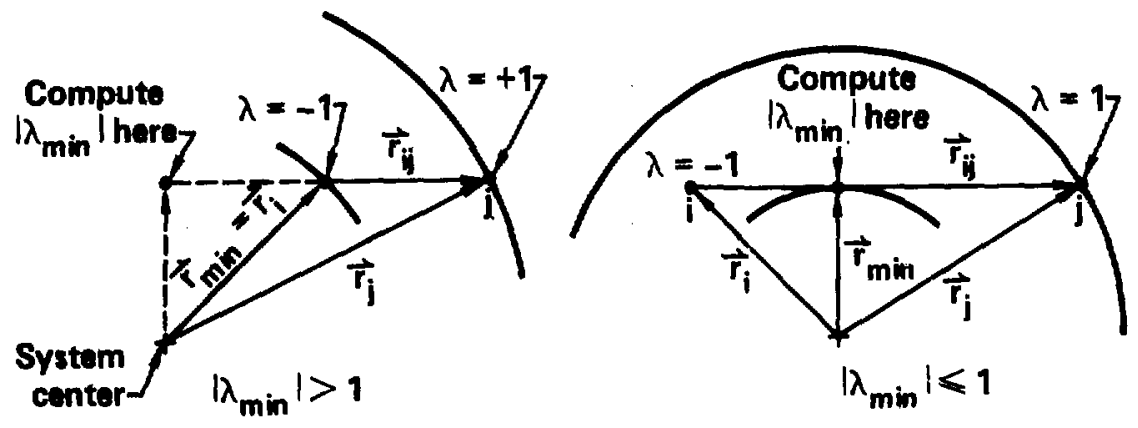

$$
\vec{r}=\frac{1}{2}\left(\overrightarrow{r_{i}}+\vec{r}_{j}+\lambda \overrightarrow{r_{i j}}\right)
$$




$$
\begin{aligned}
F_{k} & =\left|\hat{r} \cdot \hat{r}_{1 j}\right|\left(-\frac{\partial q_{1 j}}{\partial r_{1 j}}\right) \\
& =-\left|\vec{r} \cdot \vec{r}_{1 j}\right|\left(\frac{\partial q_{1 j}}{\partial r_{1 j}}\right) /\left(r_{1 j} r\right)
\end{aligned}
$$

Symmetry requires the seme contribution from each intersection,

$$
\left|\vec{r} \cdot \vec{r}_{1 j}\right|=\frac{1}{2} r_{i j}^{2}\left[\left(\frac{r_{1}^{2}-r_{j}^{2}}{r_{1 j}^{2}}\right)+1-2\left(\frac{r_{1}^{2}+r_{j}^{2}}{r_{1 j}^{2}}\right)+\frac{4 r^{2}}{r_{i j}^{2}}\right]^{1 / 2} .
$$

Th1s final term must be doubled when there are two intersections; 1.e., wisen both $\left|\lambda_{-}\right|$and $\left|\lambda_{+}\right| 1$ (Fig. 7.14). Equations 7.27 and 7.28 can be used in Eq. 7.21 to find the pressure profile. Although the instantaneous density and pressure profiles (and the liquld shape) are very irregular, time averaging produces reasonable profiles (Flg. 7.15) and a circular shape.

The pressure profile has been used to compute the surface tension in both one dimensional (planar interface) and three dimensional geonetries $^{62}$,

$$
r_{S}= \begin{cases}\int_{-}^{\infty}\left(P_{N}-P_{T}\right) d z & , 1-D \\ j\left(P_{N}-P_{T}\right)\left(\frac{r}{R_{S}}\right)^{2} d r & , 3-D .\end{cases}
$$

The same methodology is used below to derive the surface tension in two dimensions.

First, the relationship between $P_{N}, \partial P_{N} / \partial r$, and $P_{T}$ is required. Consider a two dimensional volume element as shown 
F1g. 7.14. Relationshlp between the bond and the surface.

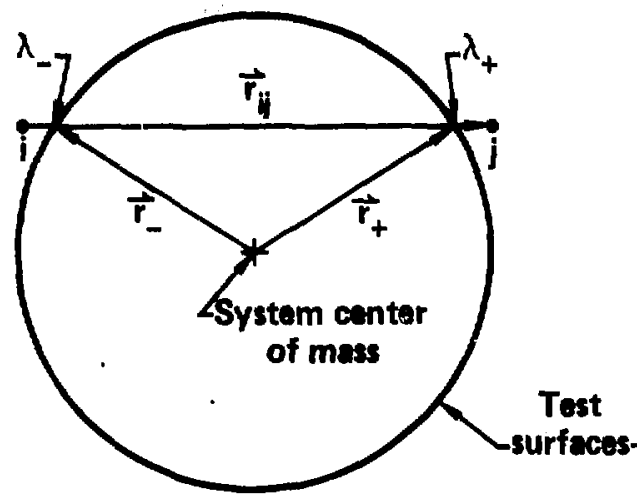

Two intersections $\mid \lambda_{+}$and $\left|\lambda_{-}\right|<1$
One intersection

$\left|\lambda_{-} \operatorname{lor}\right| \lambda_{+} \mid>1$ 
F1g. 7.15. Instantaneous atom positions and time avereged density and pressure profiles.

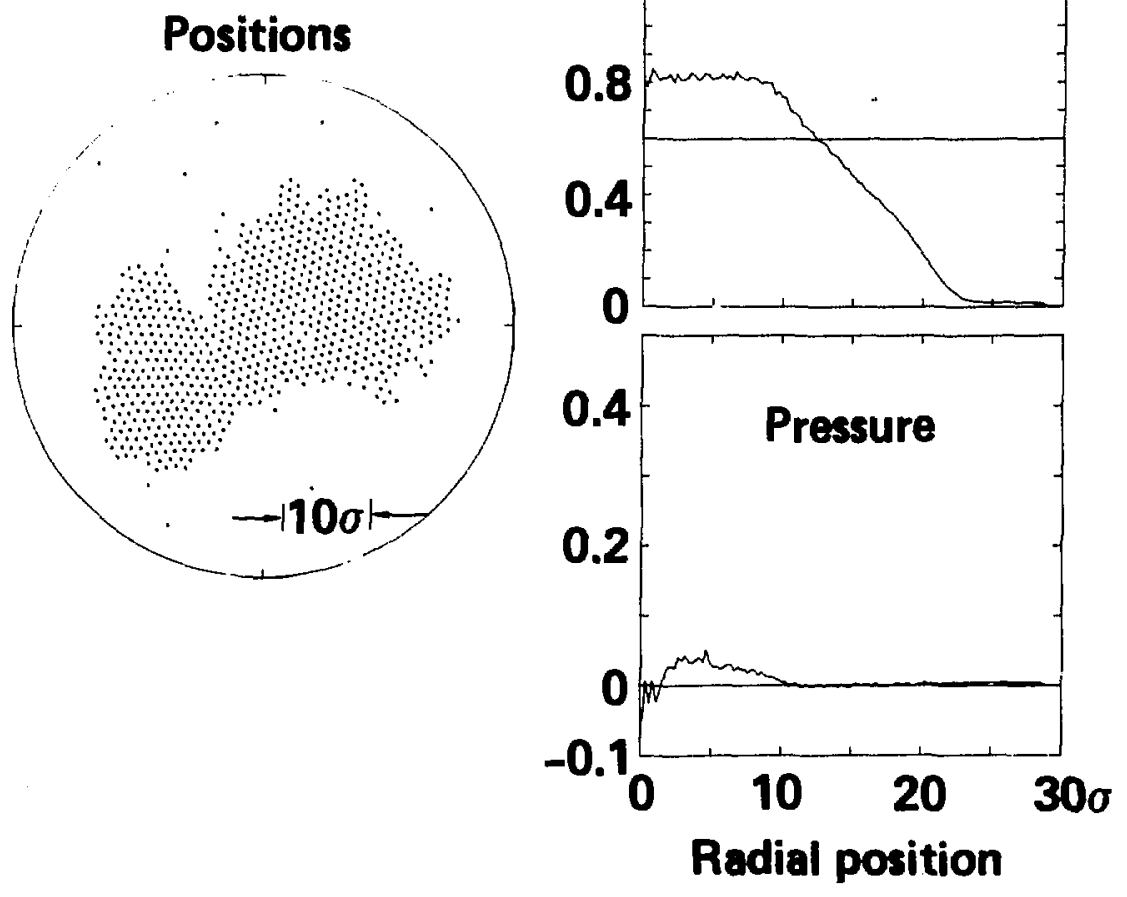


In F1g. 7.16. For zero motion,

$$
\begin{aligned}
\Sigma F_{Z} & =0 \\
& =2 P_{N} r \theta-2\left(P_{N}+\frac{\partial P_{N}}{\partial r} d r\right)(r+d r) r+2 P_{T} \sin \theta d r .
\end{aligned}
$$

For snall angles, $\sin \theta \sim \theta$, and

$$
0=-P_{N}-\frac{\partial P_{N}}{\partial r} r-\frac{\partial P_{N}}{\partial r} d r+P_{T}
$$

The or term can be made arbitrarlly small, thus,

$$
P_{T}=P_{N}+r \frac{\partial P_{N}}{\partial r}
$$

The comparable expression in three dimensions is

$$
P_{T}=P_{N}+\left(\frac{T}{2}\right) \frac{\partial P_{N}}{\partial T}
$$

Equation 7.32 has the alternative forms,

$$
\begin{aligned}
& \frac{\partial}{\partial r}\left(r P_{N}\right)=P_{T} \\
& \frac{\partial}{\partial r}\left(r^{2} P_{N}\right)=r\left(P_{N}+P_{T}\right)
\end{aligned}
$$

Next, the actual tangential force on one side of a radial line is equated to the tangential forces on the same line in the hypothetical situation where the pressure profile is a step function at the surface (Fig. 7.17).

$$
\begin{aligned}
& -\int_{R_{1}}^{R_{2}} P_{T} d r=-\int_{R_{1}}^{R_{2}} P_{12} d r+r_{S} \\
& \rightarrow r_{S}=\int_{R_{1}}^{R_{2}}\left(P_{12}-P_{T}\right) d r
\end{aligned}
$$


Fig. 7.16. Two dimensional volume element used to derive the relationship between $P_{N}, 2 P_{N} / \partial r$, and $P_{T}$. All forces are shown as positive (compressive).

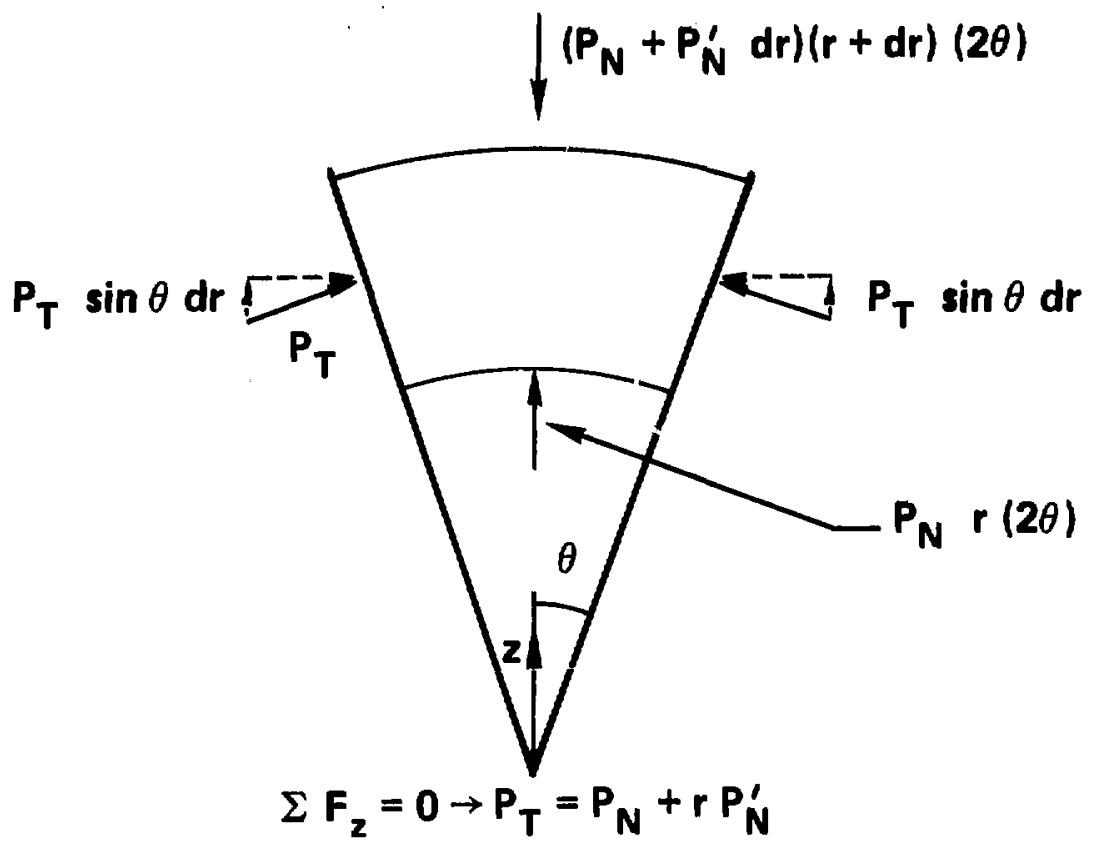


Fig. 7.17. Hypothetical strip used to determine the force balance that specifies the surface tension. The pressure arrows indicate two dimensional pressure (force/length) times incremental radius (dr) while the surface tension, $r_{s}$, has force units in two dimensions.

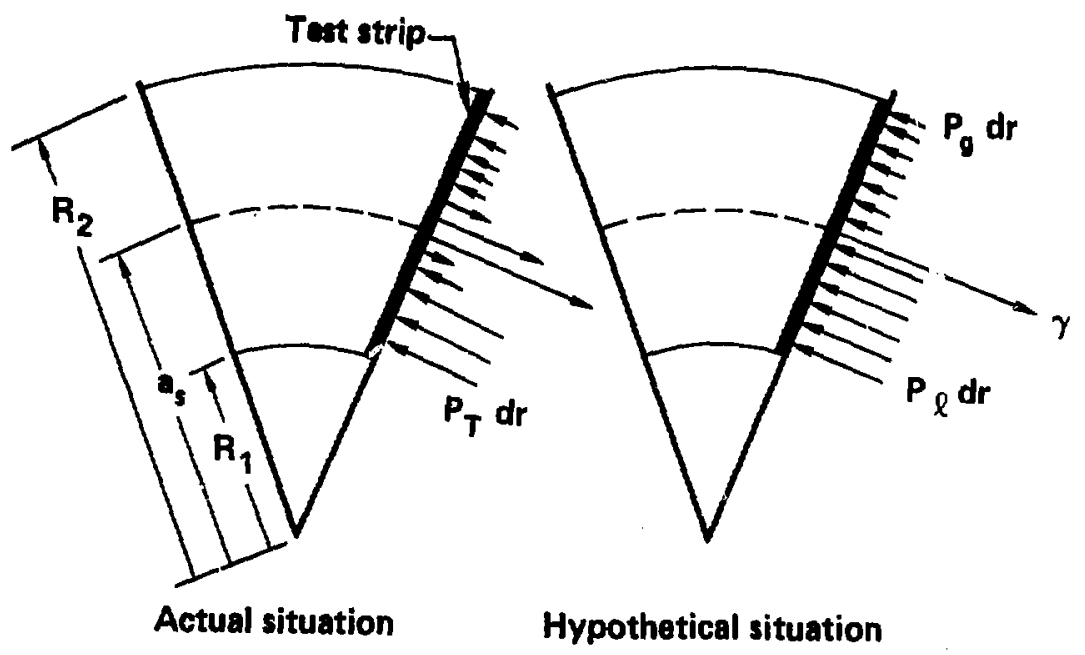


where $P_{12}$ is $P_{1}\left(P_{2}\right)$ for $r$ a and $P_{2}\left(P_{g}\right)$ for $r$ as

Similarly, the moments about $r=0$ are equated for the tirs situat tons,

$$
\begin{aligned}
& -\int_{R_{1}}^{R_{V_{2}}} P_{T} \mathrm{rdr}=-\int_{P_{1}}^{R_{2}} P_{12} \text { Idr }+Y_{S} d_{S} \\
& \rightarrow Y_{S}=\frac{1}{a_{S}} \int_{R_{1}}^{R_{2}}\left(P_{12}-P_{T}\right) r d r
\end{aligned}
$$

Next, equations 7.34 and 7.35 are integrated, and the 1sotropy of the pressure away from the interface is used.

$$
\begin{aligned}
& \int_{R_{1}}^{R_{2}} P_{T} d r=\left(\left.I P_{N}\right|_{R_{1}} ^{R_{2}}=R_{2} P_{2}-R_{1} P_{1}\right. \\
& \int_{R_{1}}^{R_{2}}\left(P_{N}+P_{. i}\right) r d r=\left(\left.r^{2} P_{N}\right|_{R_{1}} ^{R_{2}}=R_{2} P_{2}^{2}-R_{1} P_{1}^{2} .\right.
\end{aligned}
$$

These equations can be further manipulated by noting that

$$
\begin{aligned}
& \int_{R_{1}}^{R_{2}} P_{12} d r=R_{2} P_{2}-R_{1} P_{1}+a_{s} \Delta P \\
& \int_{R_{1}}^{R_{2}} P_{12} I d r=R_{2}^{2} P_{2}-R_{1}^{2} P_{1}+a_{s}^{2} \Delta P
\end{aligned}
$$

where $\Delta P \equiv P_{1}-P_{2}$. Then, Eqs. 7.38 and 7.39 become

$$
\int_{R_{1}}^{R_{2}} P_{T} d r=\int_{R_{1}}^{R_{2}} P_{12} d r-a_{s} \Delta P
$$




$$
\int_{R_{1}}^{R_{2}}\left(P_{N}+P_{T}\right) r d r=2 \int_{R_{1}}^{R_{2}} P_{12} r d r-a_{s}^{2} \Delta P
$$

The pressure difference can be eliminated from these two equations to obtaln

$$
\int_{R_{1}}^{R_{2}}\left(P_{12}-P_{T}\right) d r=\frac{1}{R_{2}} \int_{R_{1}}^{R_{2}}\left[\left(P_{12}-P_{T}\right)+\left(P_{12}-P_{N}\right)\right] r d r
$$

But, the first two terms were evaluated using the force and moment balences (Eqs. 7.36 and 7.37). Thus

$$
\gamma_{s}=\gamma_{s}+\frac{1}{a_{s}} \int_{R_{1}}^{R_{2}}\left(P_{12}-P_{N}\right) r d r
$$

Since the last term is clearly zero, its slon can be reversed. Then, Eq. 7.44 becomes

$$
r_{s}=\int_{R_{1}}^{R_{2}}\left(P_{12}-P_{T}\right) d r=\frac{1}{a_{S}} \int_{R_{1}}^{R_{2}}\left[\left(P_{12}-P_{T}\right)-\left(P_{12}-P_{N}\right)\right] r d r
$$

or

$$
r_{S}=\int_{R_{1}}^{R_{2}}\left(P_{N}-P_{T}\right)\left(\frac{r}{a_{S}}\right) d r
$$

This result is parallel to the one and three dimensional equations (Eq. 7.29). In general,

$$
Y_{S}=\int_{-\infty}^{\infty}\left(P_{N}-P_{T}\right)\left(\frac{r}{S_{5}}\right)^{D-1} d r
$$

where $D$ is the number of dimensions. The extension of the limits to infinity is perwissible since $P_{N} P_{T}=0$ oxcept near the interface. 
A force balence between the inside and outsids of the drop relates $Y_{s}, a_{s}$, and $\Delta P$ (FIg. 4.17).

$$
a_{s}=(D-1) Y_{s} / \Delta P
$$

Then,

$$
r_{S}^{D}=\left(\frac{A P}{D-1}\right) \int_{-\infty}^{\infty}\left(P_{N}-P_{T}\right) r^{D-1} d r
$$

In two and three dimensions (Eqs. 7.32 and 7.33),

$$
P_{N}-P_{T}=-\left(\frac{r}{D-1}\right)\left(\frac{\partial P_{N}}{\partial r}\right)
$$

Then,

$$
r_{S}=-\left[\frac{\Delta P^{D-1}}{(D-1)^{D}}\right] \int_{D}^{\infty} \frac{\partial P_{N}}{\partial r} r d r
$$

This equation is identical to Eq. 15 (Ref. 29) in three dimensions,

$$
r_{s}^{3}=-\left[\frac{\Delta P^{2}}{8}\right] \int_{0}^{\infty} \frac{\partial P_{N}}{\partial r} r^{3} d r
$$

In two dimensions,

$$
\gamma_{S}^{2}=-\Delta P \int_{0}^{\infty} \frac{\partial P_{N}}{\partial T} r^{2} d r
$$

Th1s result can be numerically ovaluated using the time-averaged pressure profile obtained from the molecular dynamics calculation (Eqs. 7.21, 7.27, and 7.28).

A series of two dimensional molecular dymentcs calculations was performed on systems ranging from 70 to 700 atoms at temperatures below $(0.25)$ the triple point, near it $(0.35$ to 0.4$)$, and above it 
(0.45). Time averages were taken for up to 1450 reduced time units

$(t \sqrt{c} m / \sigma)$ in a few cases, with typical averaging perlods being 400 to 700 time units.

Analysis of the results indicates that the sub-triple point runs hed not reached equilibrium. (Low densities and negative pressures typical of the two-phase solid-vapor reglon were measured.) For the hloh temperature runs (whish were only about 0.1 reduced temperature units below the critical point of $\sim 0.55$ ), fluctuations and occasional drop fragnentation obscured the results. Fortunately, near the triple polnt, reasonable results were obtained. This experience, which used - 6 CRAY CPU hours, indicates that the relatively small 2-D temperature range between the triple and critical points makes 2-D free-surface simulation very difficult.

Once a reasonable two-dimensional surface tension vs. system size curve was generated, scaling arguments were used to convert the three dimensional results to two dimensioris. A simple way of thinking about surface tension is in terms of excess energy due to missing bonds for surface atons. Then,

$$
Y=\left(\frac{\text { ulssing bonds }}{\text { surf. atom }}\right)\left(\frac{\text { surf. atoms }}{\text { surf. area }}\right)\left(\frac{\text { eneray }}{\text { bond }}\right)=\left(\frac{\text { eneray }}{\text { surf. area }}\right) .
$$

In two dinensions,

$$
r_{2}=2\left(\frac{1}{r_{2}}\right)\left(-t_{2}\right)
$$

In three dimensions,

$$
r_{3}=3\left(\frac{2}{\sqrt{3} r_{3}^{2}}\right)\left(-\diamond_{3}\right)
$$


where $r_{3}$ is the bond length for the given density $\left(w^{3}\right)$. For equal densitiles, $r_{2}$ does not equal $r_{3}$ since $p_{3}=\sqrt{2} / r_{3}^{3}$ and $p_{2}=2 /\left(\sqrt{3} r_{2}^{2}\right)$.

Application of Eq. 7.56 at $T_{2}=0.41(0=0.75)$ gives $r_{3}=1.84$, about four times the correct value of 0.43 . The simple model is expected to produce high values of surface tension becense the actual (smooth) density proflle Increases the energies of the remaining bonds; the simple model assumes a step docrease in density with no change in bond strength.

Using the density-bond length relationships and Eqs. 4.15, 7.55, and 7.56 allows calculation of the ratio of $3-D$ to $2-D$ surface tensions,

$$
\frac{r_{3}}{r_{2}}=1.14 p^{-5 / 6}\left[\left(\frac{e^{2}}{2}-1\right) /\left(\frac{p^{3}}{1.54}-1\right)\right]
$$

As before, reduced units are used $\left(\rho=\rho_{3} \sigma^{3}=\rho_{2} \sigma^{2}, \phi=\phi / c, r=r / \sigma\right.$, $T=k T / \varepsilon, \gamma_{3}=\gamma \sigma^{2} / c$, and $\left.\gamma_{2}=\gamma \sigma / c\right)$. The ratio, $\gamma_{3} / \gamma_{2}$, varies from 1.9 at $p=0.5$ to 1.4 at $p=0.9$.

Figure 7.18 shows the results of the two dimensional molecular dynamics calculations at $T=0.35$. The figure also shows the two dimensional planar limits (scaled from three dimensional values) and the $T=0.41$ two dimensional surface tension scaled from the $T=0.80$ three dimensional data. (The temperatures correspond to a reduced density of 0.75 in both dimensions.) For comparison, the predicted $T=0.41$ surface tension curve is shown assuming the two dimensional surface tension varles linearly with temperature to zero at the critlcal temperature $(T=0.56)$. The accuracy of this assumption is 
Fig. 7.18. Two dimensional surface tension from molecular dynamics calculations and from scaled three dimensional values. The data is plotted as a function of $1 / \mathrm{NN}_{\ell}$ because surface tension is expected to have a size dependence.

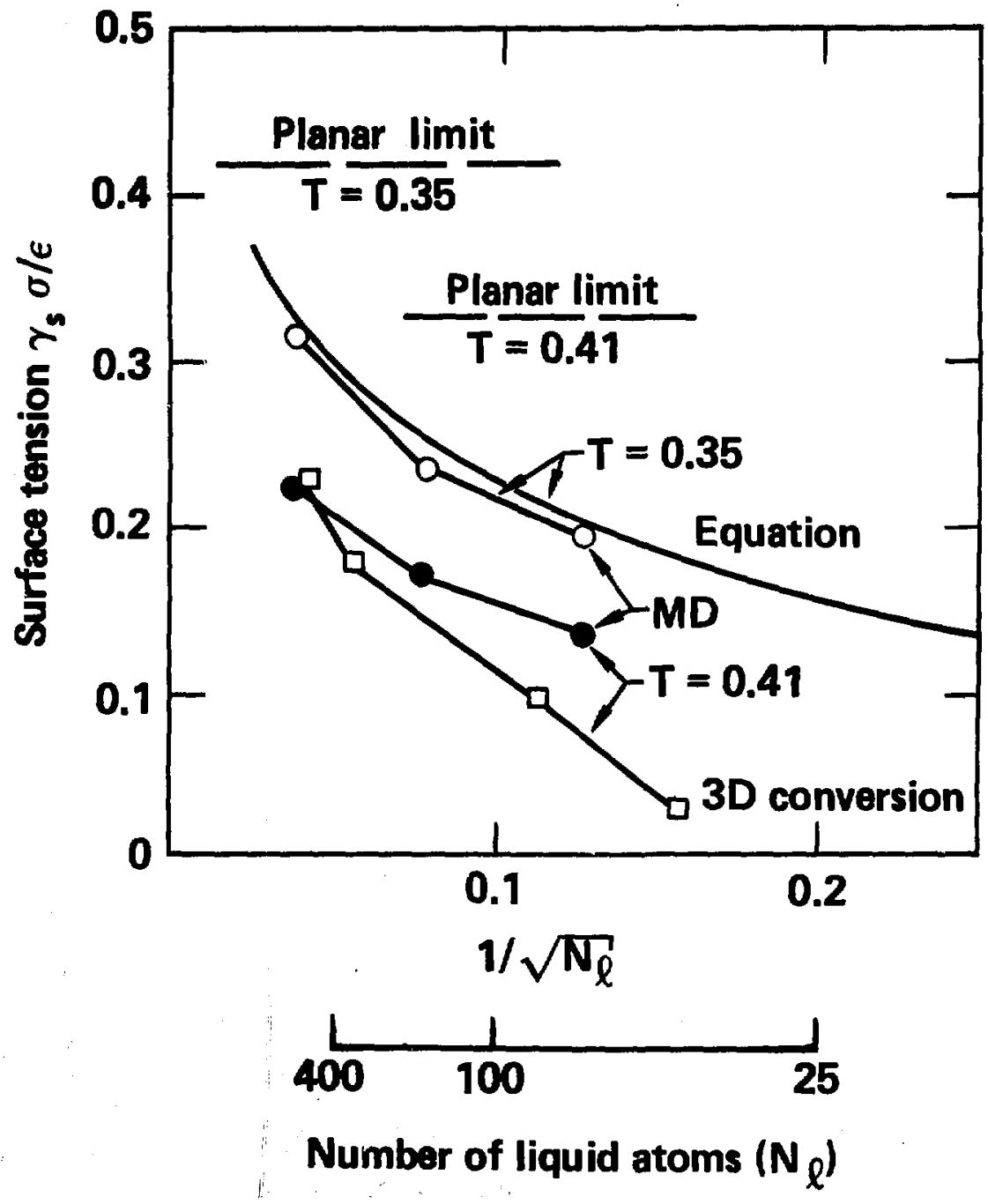


unimportant beceuse the expansion arid vapor pressure formation have reduced the temperature of most fragnents to the 0.35 region at the tine of fragmentation. Finally, the figure shows an analytical equation used to approximate the surface tension,

$$
r=0.42\left[1-\left(\frac{T-0.35}{0.21}\right)\right]\left[0.281 \log N_{l}-0.029\right]
$$

where $N_{l}$ is the number of 11quid atoms and 0.42 is the surface tension for $\mathrm{N}_{2}>5000$ atoms at a temperature of 0.35 . The equation is plotted at $T=0.35$.

Because the surface tension is st1ll somewhat uncertain, Eq. 7.58 is used, and the sensitivity to a factor of ten error is evaluated in the comparison between the molecular dynamics fragmentation xuns and the four models from Chapter Five. Equation 7.58 has also been reformulated to eliminate the logarithmic dependence in favor of a more physically meaningful power-law relationship,

$$
Y=0.35\left[1-\left(N_{2}\right)^{-1 / 2}\right]^{4}\left[1-\left(\frac{T-0.35}{0.21}\right)\right] .
$$

This reiationship is plotted in Appendix $A$, and it is not much different than Eq. 7.58.

As an alternative method of evaluating surface tension, the potential energy per atom was examined as a function of drop size for all the fragnents with more than 19 atons in the 169, 721, and 2611 atom fragmentation runs. The energy data were taken at the latest time avallable in each rin. It was hoped that the ensemble would produce an energy curve that, coupled with reasonable entropy dependence on drop size ${ }^{36}$, could be fit to a single equation with 
surface tension being one of the fitting constants. However, the scatter in the energy data (FIg. 7.19) is too large to allow an eccurate curve fic, probably because tine averages were not used. The scatter did not disappear when the kinetic energy and surface energy (from Eq. 7.58) mere added, confirming the need to time average. Unfortunately, the energy data were only compiled at infrequent Intervals. 
Fig. 7.19. Potential energy per atom as a function of drop size at times long after fregmentation. The scattered data does not allow. determination of the surfece tension by curw fitting these non-tine-averaged data.

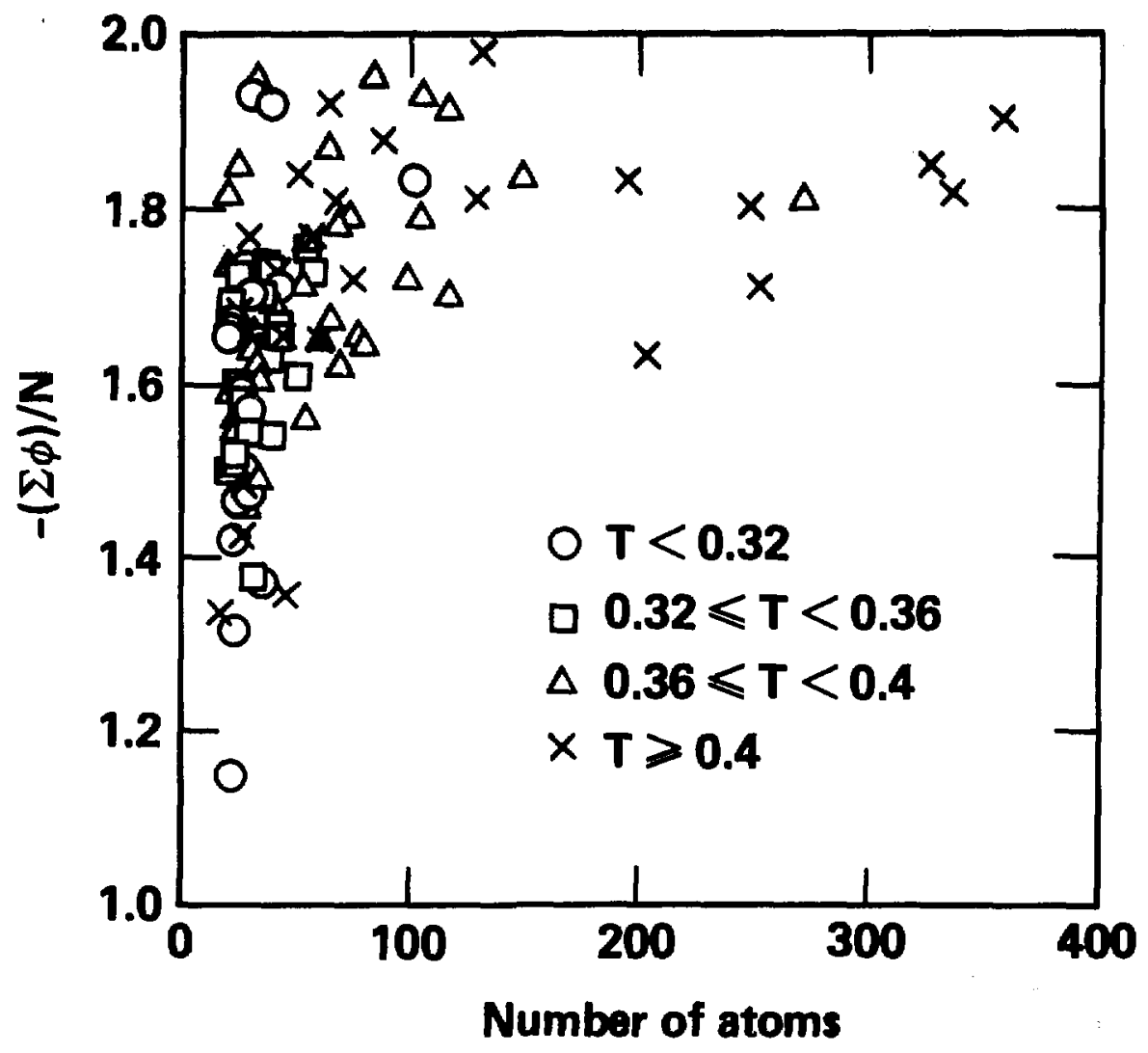


7.3. Molecular Dynamics Experiment Results

As a part of this study, nolecular dynamics calculations were carried out on two dimensional circular liquid regions of up to 14491 atoms. These liquid reglons were equilibrated within a confining wall at various temperatures batween saturation $(\sim 0.5)$ and $\sim 1.2$ and then released. An additional three dimensional molecular dynamics study ${ }^{63}$ of 250 atom cubical systems is discussed. This study equillbrated to varlous densities and temperatures using perlodic boundary conditlons and then released the boundarles. 


\subsubsection{Two Dimensional Molecular Dynamics Results}

A series of fragnentation calculations begen with wall-confined systems of $14491,2611,721$ and 169 atons that had been equllibrated at temperatures ranging from $\sim 1.2$ down to near saturation $(\sim 0.5$ to 0.55$)$. Altiough the calculations were intended to begin at constant density $(\sim 0.6)$, Inspection of the cumulative density proflle

$$
\rho_{c}(r)=\int_{0}^{r} \rho(r) 2 \pi r d r / \pi r^{2}
$$

indicated initial densities ranging from 0.61 to 0.73 . The initial conditions for the 13 fragmentation runs are shown in Table 7.2 and in Fig. 7.20.

As time evalved, snapshots of the atomic positions were used to visualize the fragmentation; an example sequence is shown in Fig. 7.21. A more quant1tative description of the fragmentation process is contalned in the next several flgures which show four fragmentation quantities as a function of time, system population and heating level. The abscissa of each plot is the time divided by the pressure relief $t 1 m e(t / t=t c / R)$. Four system slzes $(14491,2611,721$ and 169 atoms) are shown on each figure. The multiple curves for each systen size indicate different anounts of isochoric heating. The amount of heating can be seen from the $y$-intercepts on Fig. 7.22 wich shows the evolution of the thermal and translational tenperaturias with time. To decrease the impact of vapor pressure formation on the results, both temperatures are based on the total non-monomer atoms in the system. The transiational temperature is defined as the total of 
Table 7.2. Initial conditions for the fragmentation runs. Reduced units are used: $r / \sigma, k T / \varepsilon, \rho \sigma^{2}, P \sigma^{2} / c, c / m / t$, and $t_{r}=(R / c)(\sqrt{c / m} / \sigma)$.

\begin{tabular}{|c|c|c|c|c|c|c|}
\hline Population & Radius & Temperature & Density & Pressure & $\begin{array}{l}\text { Sound } \\
\text { Spend }\end{array}$ & $\begin{array}{c}\text { Rellef } \\
\text { Till }\end{array}$ \\
\hline 14491 & 86.7 & 1.01 & 0.61 & 1.02 & 3.7 & 23.6 \\
\hline $\begin{array}{l}2611 \\
2611 \\
2611\end{array}$ & $\begin{array}{l}36.6 \\
36.6 \\
36.6\end{array}$ & $\begin{array}{l}1.16 \\
0.76 \\
0.65\end{array}$ & $\begin{array}{l}0.62 \\
0.62 \\
0.62\end{array}$ & $\begin{array}{l}1.38 \\
0.53 \\
0.31\end{array}$ & $\begin{array}{l}4.1 \\
3.1 \\
2.7\end{array}$ & $\begin{array}{r}8.9 \\
11.9 \\
13.4\end{array}$ \\
\hline $\begin{array}{l}721 \\
721 \\
721 \\
721 \\
721\end{array}$ & $\begin{array}{l}19.4 \\
19.1 \\
18.8 \\
18.6 \\
18.4\end{array}$ & $\begin{array}{l}1.17 \\
0.77 \\
0.65 \\
0.55 \\
0.51\end{array}$ & $\begin{array}{l}0.61 \\
0.63 \\
0.65 \\
0.66 \\
0.68\end{array}$ & $\begin{array}{l}1.31 \\
0.60 \\
0.40 \\
0.17 \\
0.07\end{array}$ & $\begin{array}{l}4.0 \\
3.2 \\
3.2 \\
2.9 \\
2.9\end{array}$ & $\begin{array}{l}4.8 \\
5.9 \\
6.0 \\
6.4 \\
6.2\end{array}$ \\
\hline $\begin{array}{l}169 \\
169 \\
169 \\
169\end{array}$ & $\begin{array}{l}9.1 \\
8.9 \\
8.9 \\
8.6\end{array}$ & $\begin{array}{l}1.19 \\
0.77 \\
0.62 \\
0.50\end{array}$ & $\begin{array}{l}0.65 \\
0.68 \\
0.68 \\
0.73\end{array}$ & $\begin{array}{l}1.75 \\
0.86 \\
0.41 \\
0.11\end{array}$ & $\begin{array}{l}4.6 \\
4.0 \\
3.5 \\
3.6\end{array}$ & $\begin{array}{l}2.0 \\
2.3 \\
2.6 \\
2.4\end{array}$ \\
\hline
\end{tabular}


Fig. 7.20. Initlal conditions for the two dimensional molecular dynemlcs fragmentation calculations.

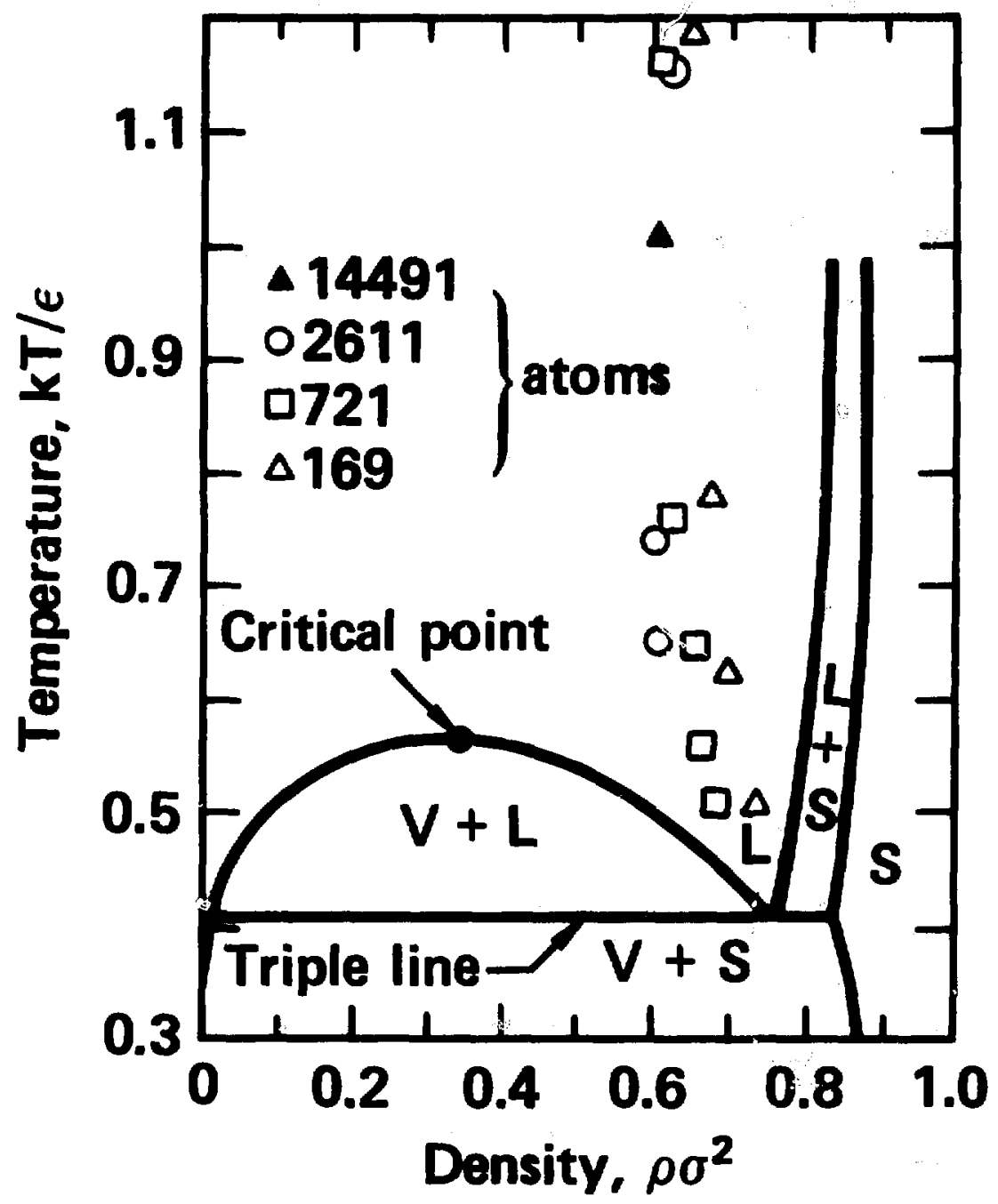


F1g. 7.21. Snepshots of the atomlc configurations at four times during the fragnentation process of a 14491 atom system $(T \sim 1.0)$.

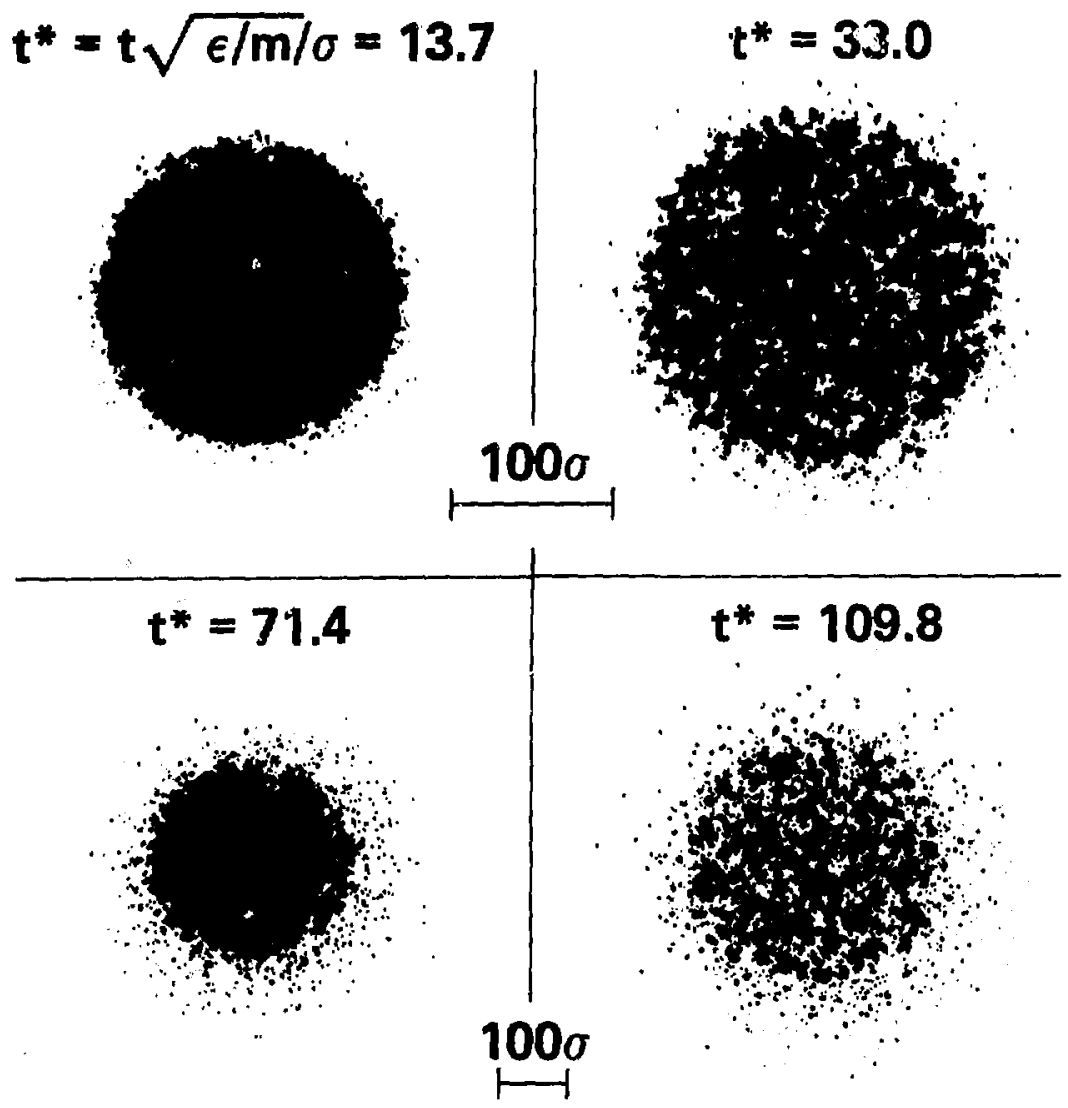


Fig. 7.22. Thermal and translational temperature evolution in the 2-D fragnentation runs.

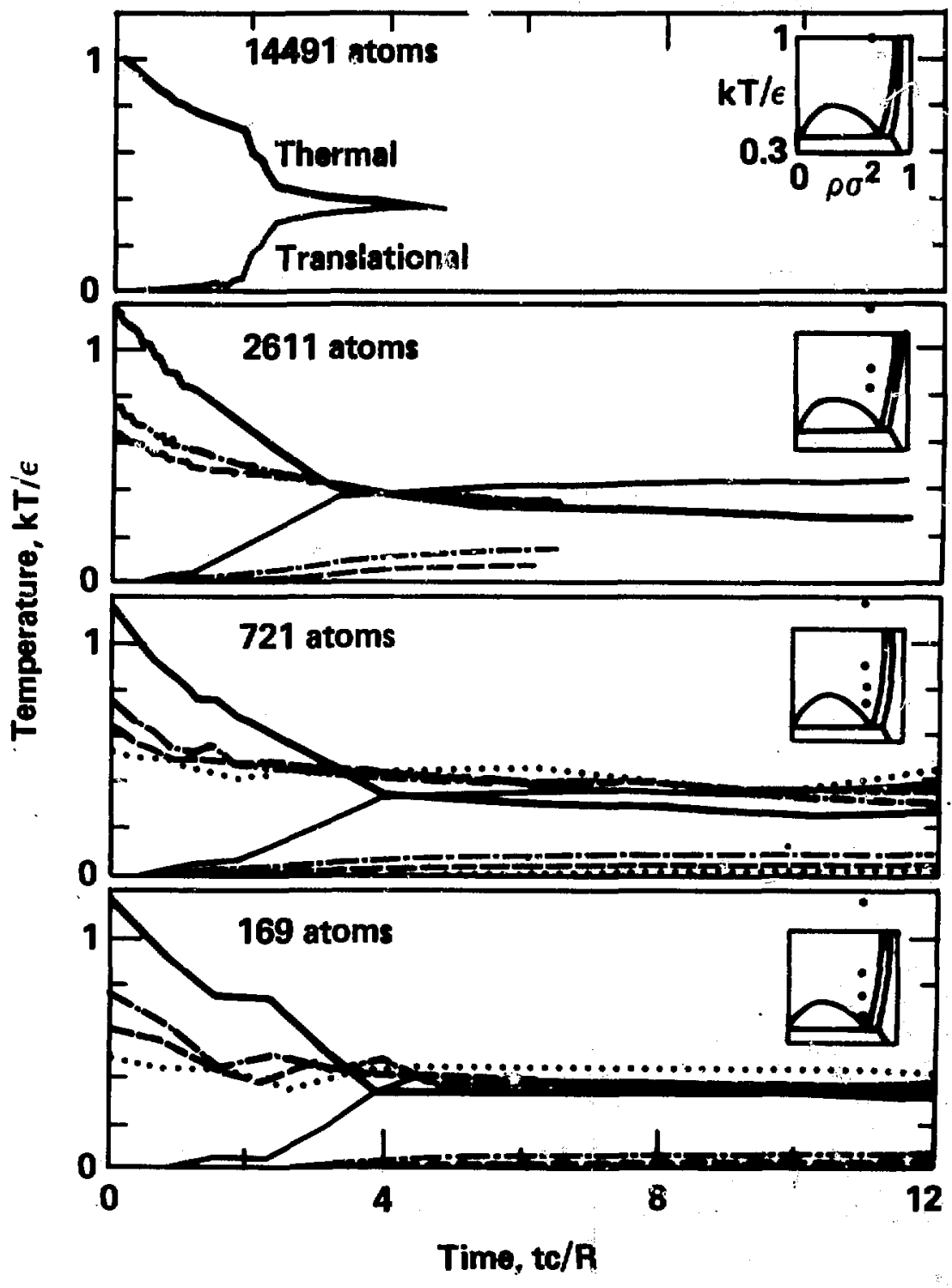


the center of mass kinetic energles of all the clusters with more then one atom, divided by the number of non-monomer atoms. The thermal temperature is defined as the total kinetic energy of non-monomer atoms less the cluster center of mass translational and rotational energles, all divided by the number of non-monomer atons. The rotational temperature, defined by $T_{\operatorname{rot}}=2\left(L^{2} / 2 N_{c} I\right)$ (Section 6.8.2), was not plotted because it was always too low to show on the graphs.

The thermal temperature relaxed to a "post-fragnentation" level of cbout 0.35 to 0.4 (near the triple point) after about two or three pressure relief times (R/C) in all cases (FIg. 7.22). The trenslational temperature reached its "post-fragnentation" value at obout the same tine; however, the value was very dependent on the initial "pre-fragmentation" thermal temperature. For the hlohest hating level (to $T \sim 1.15$ ), the final translational temperature was - 0.4, somowhat higher than the final thermal temperature. For the lower hating levels, the final translational temperature was 0.1 or lower. There was an observable system slze dependence for the trenslational temperature, with the larger systens attaining hlgher values. (Increased fragmentation is expected from the lower surface to volum ratlos in largar systens as show in Chapter Five.) The total of the final translational and thermal temperatures was always less then the initial thermal temperature; the remaining energy was carrled as kinetic energy by monomer atoms in the vapor, or stored as potential eneroy in the interatomic bonds. The system potential eneray increased due to bond loss during both vapor pressure formation and surface area growth (fregmentation), and decreased due to the 
increase in bulk density caused by the lower liquid temperature. (The increases in the potential energy were higher in monitude then the decreases.)

The thermal temperature of the largest cluster is shown in Fig. 7.23. Comparison with F10. 7.22 shows that the averege and largast cluster temperatures were similar at all tims.

The fractional size of the largst cluster is shown in Fig. 7.24. inen fragmentation was axtensive, it occured at about two to four pressure reliof times. In some cases, fragmentation did not occur (small hating in smil systems). The top curve $(T \sim 0.55)$ in the 721 atom graph is interesting; between 7.5 and 9.5 pressure relief times, about $20 \%$ of the atoms fragnented, but by the next compliation time $(t / \tau$ 11.5), the fragment had been recaptured due to its internal oscillation (which caused an elongation). The $T \sim 0.75$ runs appear to be borderline for the three smallest system sizes, with fragmentation occuring in steps over an extended period of time much longer than the thermal temperature relaxation time.

The largest cluster size is replotted in Fig. 7.25 using a normalizing factor from model $3\left(1 / \mathrm{N}^{2 / 3}\right)$. If model 3 is correct, the curves starting from siallar temperatures should coalesce after fragmentation. The larger degree of success of the normalization for high initial temperatures indicates that the model works best, for vigorous fragmentation, and not as well for marginal fragmentation. The final figure of the series, Fig. 7.26, shows the bond count per non-monomer atom. In each case, the initial values of five to six included $\sim 3$ first neighbor and $\sim 3$ second meighbor bonds per atom. The bond count fell due to fragmentation and vapor pressure 
F1g. 7.23. Thermal temperature evolution of the largest cluster in the 2-D fragmentation runs. (The cluster identity sometimes changed with time.)

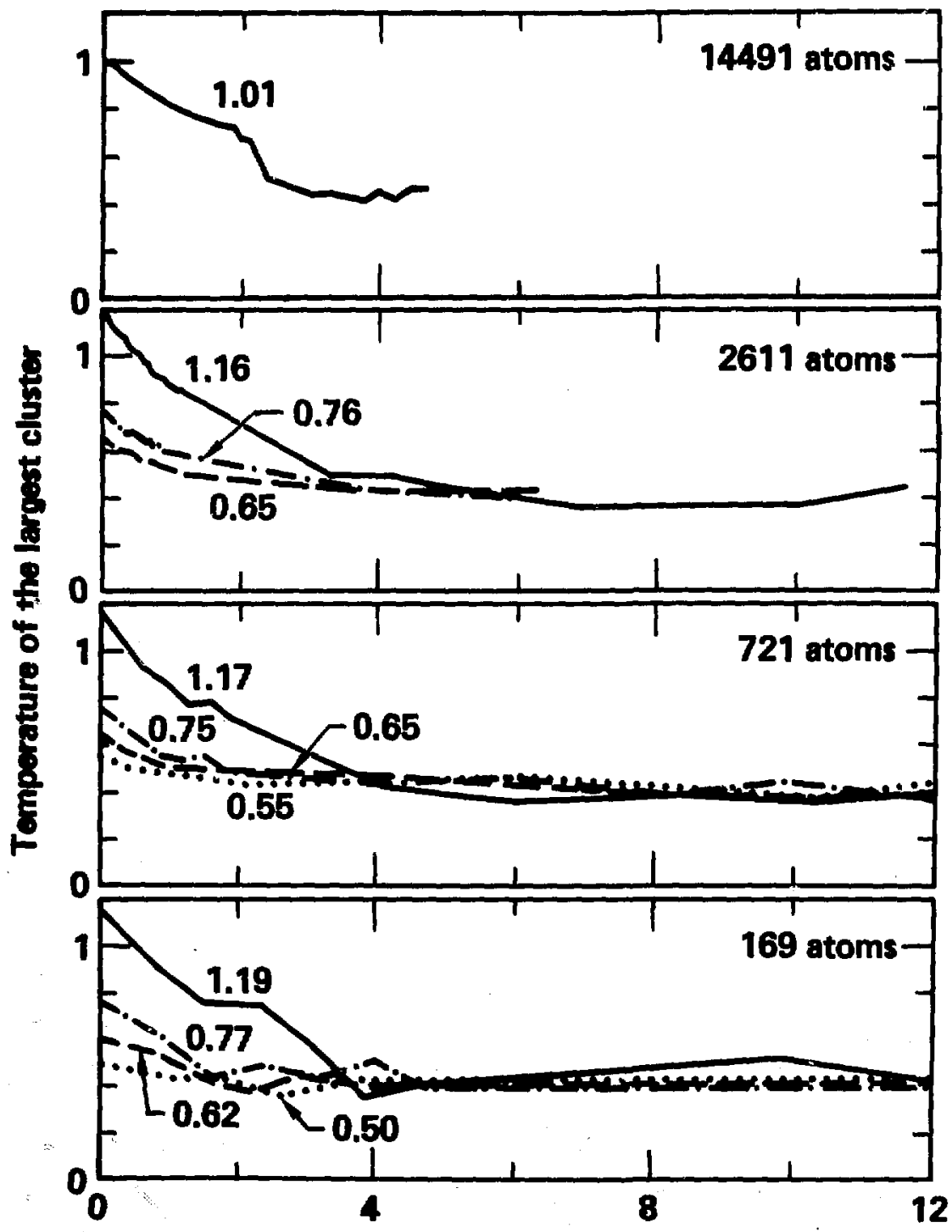


Fig. 7.24. Fractional size of the largest cluster in the 2-D fragnentation runs (size / non-monomer population). The cluster Identity sonetines changed with time.

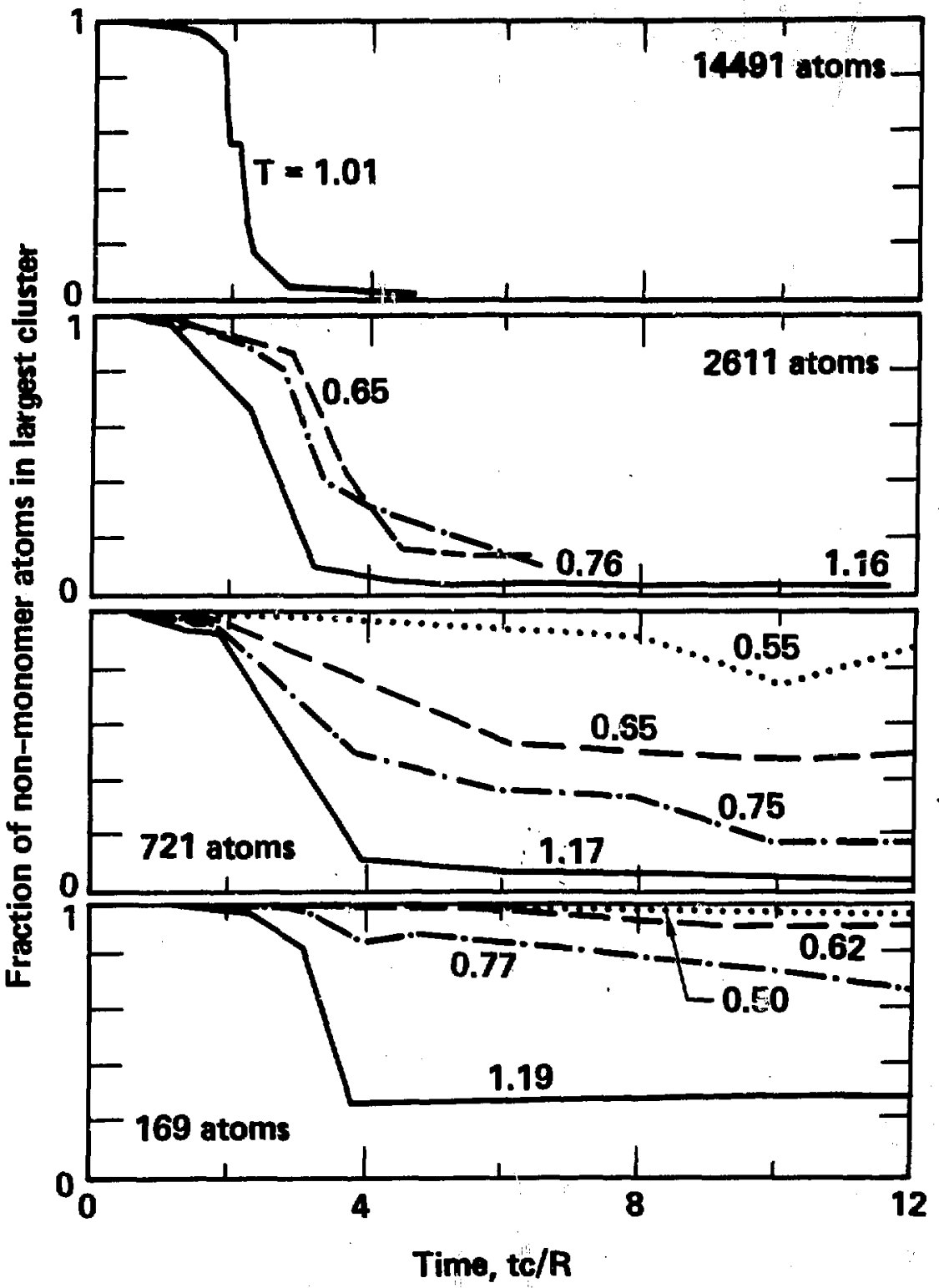


Fig. 7.25. Normalized size of the largest cluster in the 2-D frequentation runs (size / system $s i z e^{2 / 3}$ ). The normalization (fron modal 3) should cause the curves with similar tomperatures to coalesce after fragmentation. When fragmentation was extensive, the curves do coalesce, supporting the validity of nodel 3.

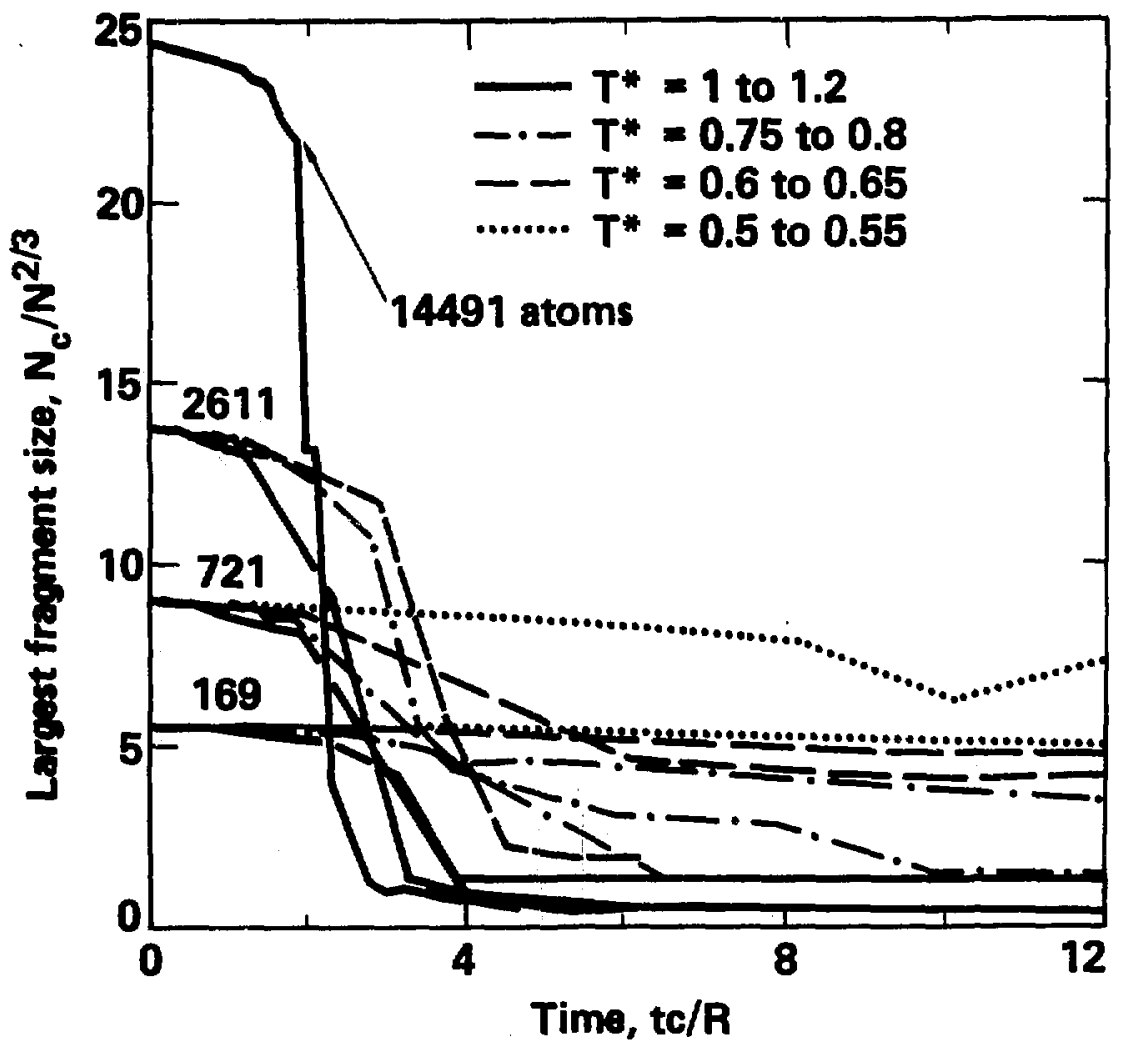


Fig. 7.26. Bonds per non-monomer atom in the 2-0 fregmentation runs.

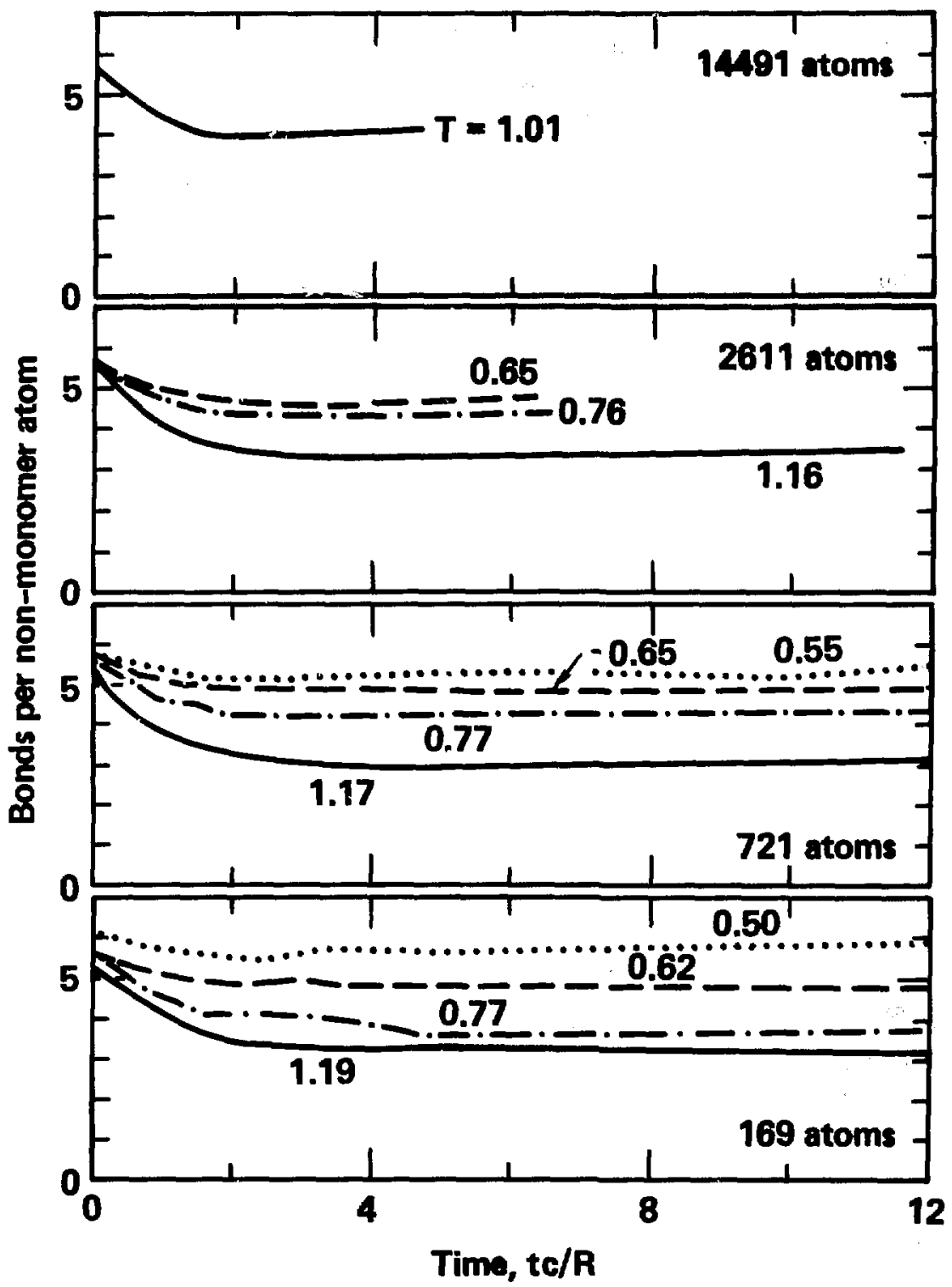


formation in about the same time as the temperature; "post-fragmentation" values were reached in two to four pressure relief times. (Since the temperature change was independent of the potential truncation distance, the temporal agreement between the temperature, the cluster size and the bond count indicates that the truncation distance was not inappropriate.) For the borderline cases $(T \sim 0.75)$, the bond count was not affected by the late time fregmentation; this was because most of the bonds were broken in the early stages $(t / \tau \sim 2$ to 4$)$, forming Internal volds. However, the cluster separation was not complete unt1l all bonds between the clusters were broken; when the fragmentation was borderline, this occured much later due to the small translational velocities generated.

The paths followed on T-p diagram are shown in Fig. 7.27 for two 2611 atom runs. The paths shown use spatially averaged densitles up to the fragmentation point, but inspection of the position plots shows marked heterogeneity much earlier. (The definition of fragmentation used here requires a minimum fragment separation of 2.50.) Internal voids which eventually become fragment surfaces appear much earlier. The internal volds decrease the "pre-fragment" density; hence, density appears to increase upon fragmentation. Temperature also decreases; as a new daughter fragment is born, its "center-of-mass translational energy" is instantaneously subtracted from the "internal" energy of its parent cluster. Most of the fragment temperatures lie along the saturated liquid phase line. Finally, although the low temperature run appeared to penetrate the unstable region under the isothermal spinodal (and nay have reached the isentropic spinodal wich lies below the (sothermal spinodal), the 
Fig. 7.27. Pressure-density paths followed by two fragmentation runs. The densities were calculated in en average sense; ectual local density in the fluid did not reach such low values.

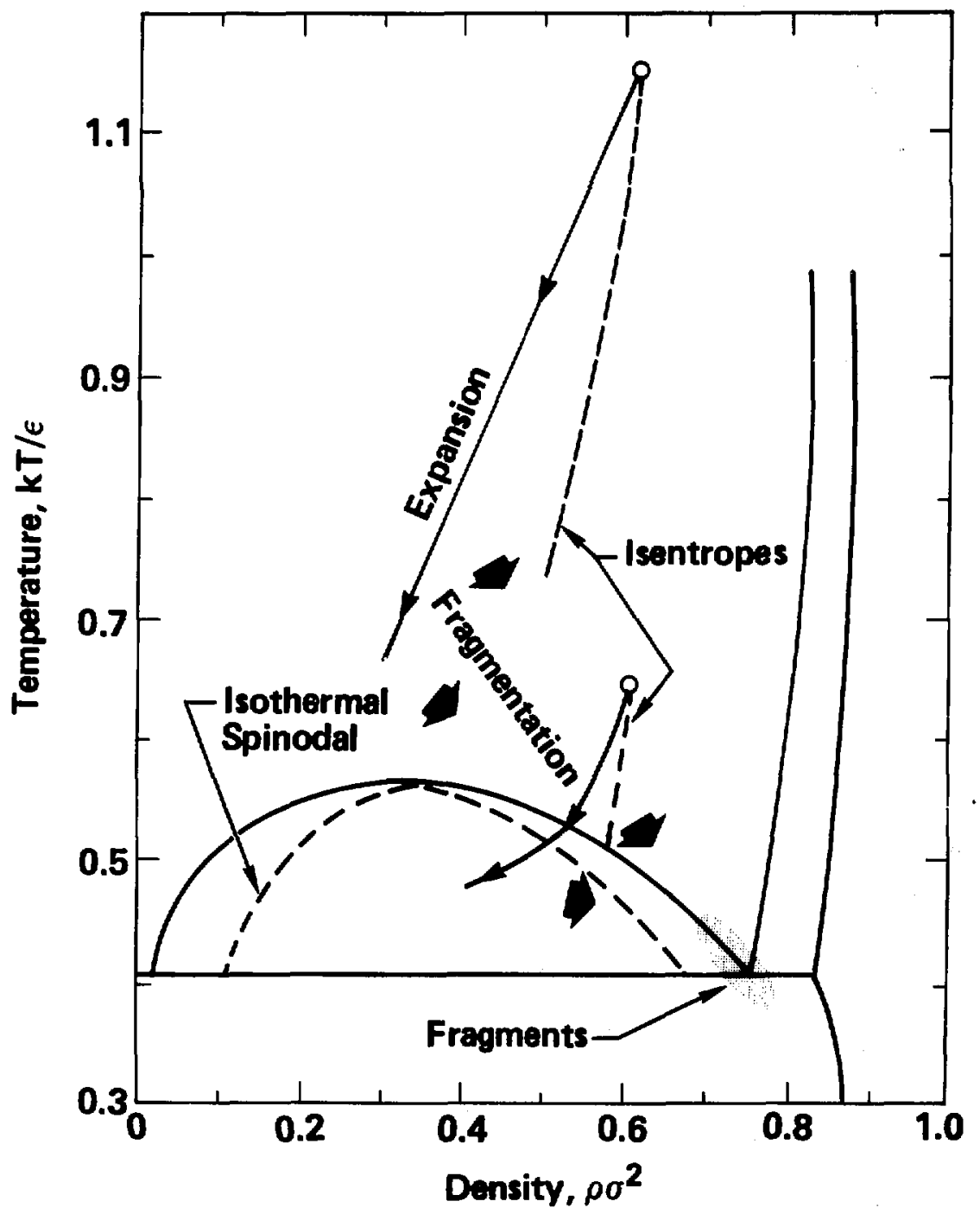


rogular density patterns ${ }^{64,65}$ characteristlc of "splinodal decomposition" were not observed. This is because the fragnentation expension is inhomogeneous. If the internal volds that precede fragmentation were not included in the density calculation, then the spinodal lines would not be reached.

The fragment size distribution for the 14491 atom run after fregunentation was ovident is shown in Fig. 7.28. The solid lines show the population in clusters of the given size. The curves are the population in the bin (e.g. 10 to 14 atoms) divided by the bin width (e.g. 4 atons). The abscissa is the fragment size divided by the total population; hence, the integral of each solid curve is 1.0 . The jagged curve includes only one fragment size in each bin, but variable width bins are used to eliminate narrow spikes in a mostly-zero background. The smoother curve has wider bins, but the same integral. This smoother curve is probably similar to an ensemble average of number of calculations beginning with slightly different configurations of identical energy and average density.

The dashed line in Fig. 7.28 shows the integrated distribution for the 14491 atom run; 1.e. each curve value indicates the fraction of the total population in fragments of the given size or smaller. The population distribution was concentrated in small fragments, with one-half of the population in fragments that were less than $20 \%$ of the slze (in atoms) of the largest fragment.

The integrated (cumulative) population distributions for three runs with similar hoating levels and varying system sizes are shown in F10. 7.29. The smilest (721 atom) system had a significant portion of the population concentrated in en intermediate fragment size range 
Flg. 7.28. Fragment size distribution for the 14491 atom system at $t=104.3$, fust after fregmentation ws well defined. The solid lines show the fractional population distribution as a function of fregunt size; the integrals of both the raw and the smothed curves are 1.0. The dashed line is the running integral of the raw curve.

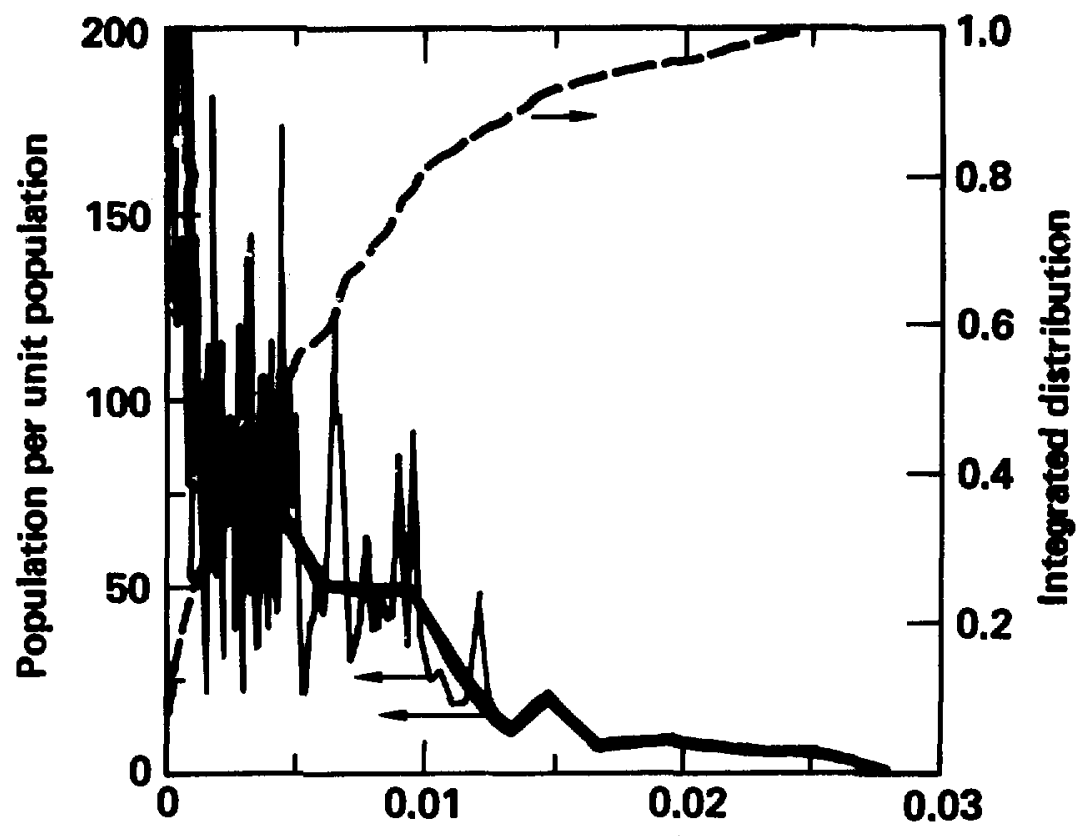

Fractional fragment population $\mathbf{N}_{c} /\left(\mathbf{N}-\mathbf{N}_{m}\right)$ 
F1g. 7.29. Integrated population distributions as a function of fragment size for three fragmentation runs with similar heating levels but difforent system sizes. The dashed lines give integrals as functions of the fractional fragnent size, $N_{c} / N$, and the solid lines are normalized to the largest fragnent size, 1.e. the abscissa is $\mathrm{N}_{c} / \mathrm{N}_{c} \max$. The fragment data were taken just as fragment sizes became woll defined.

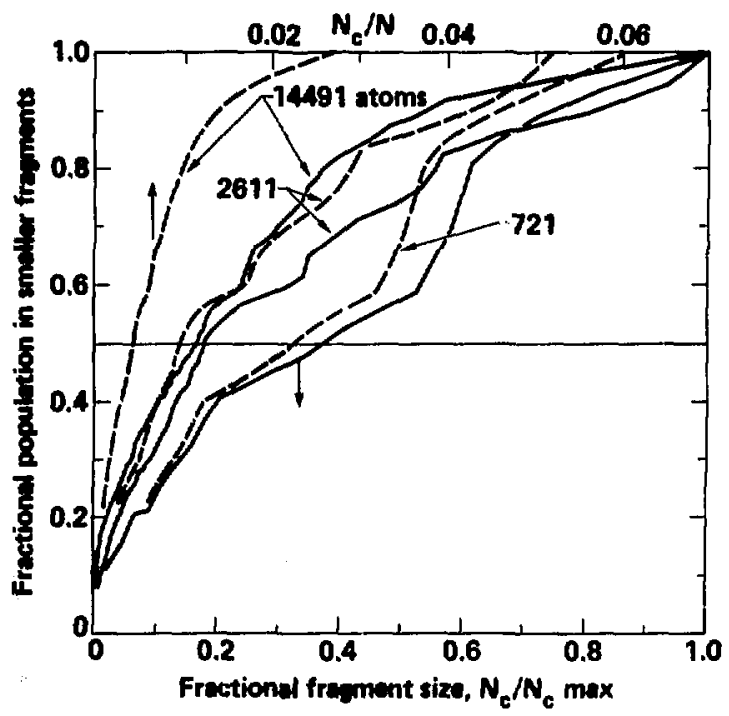


(Indicated by the steep portion of the curve). The larger systems'

pspilations were peaked at relatively lower fregment sizos and exhlbited decreasing population density with increasing fregment size. The overall effect of increasing system size was to concentrate population in smaller fractional fragment sizes; $50 \%$ of the population was in fragments smaller than 36\%, 18\%, and $17 \%$ of the largest fragment size for the 721,2611 , and 14491 atom systems, rospectively. 
7.3.2. Three Dimensional Molecular Dynamics Results.

A series of tinree dimensional molecular dynenics calculations were carried out this year by an Italian and University of Illinois tean $^{63}$. Each calculation began with perjodic boundary conditions and a cubical 250 argon atom system (using the 6-12 Lennard-Jones. potentlal with a cutoff at $I=3 \sigma$ ). The atons were initlally at the lattice points of a bndy-centered-cubic structure. The specific volume was set and maintained at 12,24 or $48 \mathrm{~cm}^{3} /$ mole during equilibration from an initial randomly generated velocity distribution corresponding to $300,600,1200$ or $2400 \mathrm{~K}$. After 600 time steps (D.DI ps each) of constant eneigy equilibration, the periodic boundary conditions were released. The resulting evolution of the system was computed for 3000 time steps with energy compilations each 300 time steps. Each of the twelve runs was repeated three times to check the statistical fluctuation in the results.

Because the Itallan study was primarily concerned with the velocity distributiun in the vapor and small clusters, the authors did not report results for clusters larger than seven atoms. However, they were kind enough to privately provide full computer outputs for two runs: $V=48 \mathrm{~cm}^{3} /$ gnole, $T=300 \mathrm{~K}$; and $V=24 \mathrm{~cm}^{3} / \mathrm{gmole}$, $T=600 \mathrm{~K}$. For argon, the authors used the following paraneters:

$$
\begin{aligned}
& m=39.96 / 6.023 \times 10^{26}=6.63 \times 10^{-26} \mathrm{~kg} / \mathrm{stom}, \\
& \sigma=3.405 \times 10^{-10} \mathrm{~m} \text {, and } \\
& c / \mathrm{k}=119.8 \mathrm{~K} .
\end{aligned}
$$


From these parameters,

$$
\begin{aligned}
& c=119.8\left(1.38 \times 10^{-23}\right)=1.65 \times 10^{-21} \mathrm{~J}, \\
& \Delta t \sqrt{\varepsilon} \pi / \sigma=10^{-14} \sqrt{c / m} / \sigma=0.0046 \\
& \rho \sigma^{3}=6.023 \times 10^{23} \sigma^{3} / V\left(\mathrm{~cm}^{3} / \mathrm{gmole}\right) \\
& \left\{\begin{array}{l}
=0.495, V=48 \mathrm{~cm}^{3} / \mathrm{gmole} \\
=0.991, V=24 \mathrm{~cm}^{3} / \mathrm{gmole}
\end{array}\right. \\
& \mathrm{kT} / c=T / 119.8 \mathrm{~K} \\
& \left\{\begin{array}{l}
=2.50 \text { for } T=300 \mathrm{~K} \\
=5.01 \text { for } T=600 \mathrm{~K}
\end{array}\right.
\end{aligned}
$$

Reduced units $\left(\rho \sigma^{3}, \mathrm{KT} / \varepsilon, \mathrm{P \sigma}{ }^{3} / \varepsilon\right.$, and $\left.E / \varepsilon\right)$ are used in the remainder of this section.

The computer output included cluster size distribution, overall temperature, and both thermal and translational temperatures for each cluster size, all at 300 time step intervals (1.38 reduced time units). After some examination, the total and thermal temperatures were determined to be in units of energy per atom while the translational temperatures were in different units (energy per cluster). Using this information, the system thermal and translational temperatures were determined,

$$
\begin{aligned}
& T_{t r}=\underset{S_{c}=2}{\operatorname{largest}}\left\{\left[T_{t r}\left(S_{c}\right)\right]\left[N_{s}\right]\right\} /\left(N-N_{m}\right) \\
& T_{t h}=\sum_{S_{c}=2}^{\operatorname{largest}}\left\{\left[T_{t h}\left(S_{c}\right)\right]\left[S_{c}\right]\left[N_{s}\right]\right\} /\left(N-N_{m}\right)
\end{aligned}
$$


whore

$S_{C}$ is the number of atoms in a cluster,

$\mathrm{N}_{s}$ is the number of clusters of size $\mathrm{S}_{\mathrm{c}}$,

$N_{n}$ is the number of monomer atoms,

$N$ is the system population,

$T_{t r}$ is the raduced translational temperature for the system (on

a per atom basis),

$T_{t r}\left(S_{c}\right)$ is the reduced translational temperature for cluster

siza $S_{c}$ (on a per cluster basis).

$T_{\text {th }}$ is the reduced thermal temperature for the system (on a per atom basis), and

$T_{t h}\left(S_{c}\right)$ is the reduced thermal temperature for cluster size $S_{c}$ (on a per atom basis).

When Eqs 7.63 and 7.64 are modified to include monomers, the sum of $T_{t r}$ and $T_{t h}$ equal the system temperature given in the computer output, which verifies the equations. (If $T_{t r}\left(S_{c}\right)$ is assumed to be on a por atom basis, the total temperature increases with time, an unreasonable result.)

To allow comparison with model 3 , the pressure at boundary release thim, the wave speed, and the surface tension are required. The three dimensional surface tension was discussed in Section 7.2.3. The pressure is aveilable from a semi-enplrical expression by Ree ${ }^{66}$,

$$
\begin{aligned}
P= & \rho T+B_{10^{\rho}} \rho^{11} T^{-3 / 2}+\sum_{1}^{4} B_{1} \rho^{1+1} T^{1-0.251} \\
& -\sum_{1}^{5} 1 C_{1} \rho^{1+1} T^{0.5-0.251}+\sum_{1}^{5} A_{1} \rho^{1+1} T^{-1 / 4}
\end{aligned}
$$


where $\rho, T$, and $P$ are reduced variables, and the constents $A_{1}$ to $A_{5}, B_{1}$ to $B_{4}, B_{10}$, and $C_{1}$ to $C_{5}$ are listed in Teble 7.3.

The sound speed is calculated from Eq. 7.18. Ree's pressure equation can be used to determine the quantitles needed to ovaluate Eq. 7.18, $(\partial P / \partial P)_{T},(\partial P / \partial T)_{P}$, and $(\partial E / \partial T)_{P}$.

$$
\begin{aligned}
\left(\frac{\partial P}{\partial \rho}\right)_{T}= & T+{ }_{11 B_{10} \rho^{10} T^{-3 / 2}+\sum_{1}^{4}(1+1) B_{1} \rho^{1} T^{1-0.251}} \\
& \sum_{1}^{5} 1(1+1) C_{1} \rho^{1} T^{0.5-0.251}+\sum_{1}^{5}(1+1) A_{1} \rho^{1} T^{-1 / 4} \\
\left(\frac{\partial P}{\partial T}\right)_{\rho}= & \rho-1.58_{10^{\rho}} \rho^{11} T^{-5 / 2}+\sum_{1}^{4}(1-0.251) B_{1} \rho^{1+1} T^{-1 / 4} \\
& -\sum_{1}^{5} \frac{1}{2}(1-0.51) C_{1} \rho^{1+1} T^{-0.5-0.251}-\sum_{1}^{5} \frac{1}{4} A_{1} \rho^{1+1} T^{-1-0.251} .
\end{aligned}
$$

The Internal energy is

$$
E=(3 / 2) \rho T+\partial\left(A^{e} / T\right) / \partial(1 / T)
$$

where the excess Helnholtz free energy per atom is

$$
A^{e}=\frac{T}{\rho} \int_{0}^{\rho}\left(\frac{P}{\rho T}-1\right) d \rho
$$

Combining Eqs. $7.65,7.68$ and $7.69 ;$ and then differentlating produces

$$
\begin{aligned}
\left(\frac{\partial E}{\partial T}\right)_{\rho}= & \frac{3}{2} \rho+T-\frac{3}{22} B_{10} \rho^{10} T^{-5 / 2}+\sum\left(\frac{1-0.251}{1+1}\right) B_{1} \rho^{1} T^{-1 / 4} \\
& -\frac{5}{1}\left(\frac{1-0.51}{1+1}\right) \frac{1}{2} C_{1} \rho^{1} T^{-0.5-0.251}-\sum_{1}^{5\left(\frac{1}{4(1+1)}\right) A_{1} \rho^{1} T^{-1-0.251}} .
\end{aligned}
$$


Table 7.3. Constents used in Ree's ${ }^{66}$ pressure equation. ( $A_{1}$ to $A_{5}$ were labeled $D_{1}$ to $D_{5}$ by Ree).
$\mathrm{B}_{1}=3.629$
$C_{1}=5.3692$
$A_{1}=-3.4921$
$\mathrm{B}_{2}=7.2641$
$C_{2}=6.5797$
$A_{2}=18.6980$
$B_{3}=10.4924$
$C_{3}=6.1745$
$A_{3}=-35.5049$
$\mathrm{B}_{4}=11.459$
$C_{4}=-4.2685$
$A_{4}=31.8151$
$B_{10}=2.17619$
$c_{5}=1.6841$
$A_{5}=-11.1953$ 
to the atomic structure changing from solid to liquid (increasing the potential energy at the expense of the kinatic energy). The $T=2.50$ Iun actually began to fragment from a roduced temperature of 2.36; the $T=5.01$ run began to fragment from a temperature of 2.50 . The temperature, density, pressure, sound speed and cube half-width at the start of fragmentation are shown for both runs in Table 7.4. The cube half-width was calculated from

$$
\rho=250 \text { atons } /\left(2 L_{1 / 2}^{3}\right)
$$

The conditions at the boundary release time are shown in Fig. 7.30.

The results of the two fragmentation runs are shown in Fig. 7.31 which has the same format as Figs. 7.22, 7.23, and 7.24. The top graph shows the evolution of the system translational and thermal temperatures, the middle graph shows the thermal temperature of the largest cluster, and the bottori graph shows the size of the largest cluster. The abscissa is time divided by the expected relief wave traversal tine. In each case, monomer atons have been excluded from both the energy and the population compllations in order to reduce the Influence of vapor pressure formation on the fragmentation results.

The curves are qualitatively like their two dimensional counterparts although fragmentation was sonewhat slower. The 3-D runs were for a very small system (less than one-half the radius of the smallest, 169 atom, 2-D runs). In 2-D, the fragnentation time was relatively independent of the initial conditions, but all those runs began at about the same density. In 3-D, the hioher density system fragmented slower, probably because the denser atoms had to tiavel 
farther before reaching the 30 potentlal cutoff used to define the clusters.

Table 7.4. Thermodynamic and geometric quantilties at the start of the 3-D fragmentation runs.

$\begin{array}{lcc}\rho & 0.495 & 0.991 \\ T & 2.36 & 2.50 \\ T^{\dagger} & (2.50) & (5.01) \\ P & 1.49 & 17.89 \\ (\partial P / \partial T)_{P} & 1.25 & 6.18 \\ (\partial P / \partial P)_{T} & 6.70 & 81.71 \\ (\partial E / \partial T)_{P} & 3.72 & 5.88 \\ {\left[(\partial P / \partial P)_{T}\right]^{1 / 2}} & 2.59 & 9.04 \\ c & 3.28 & 9.91 \\ L_{1 / 2} & 3.98 & 3.16\end{array}$

t prior to equilibration 
Fig. 7.30. Initial temperatures and densities for the two Italien 3-0 molecular dynemics fragnentation calculations.

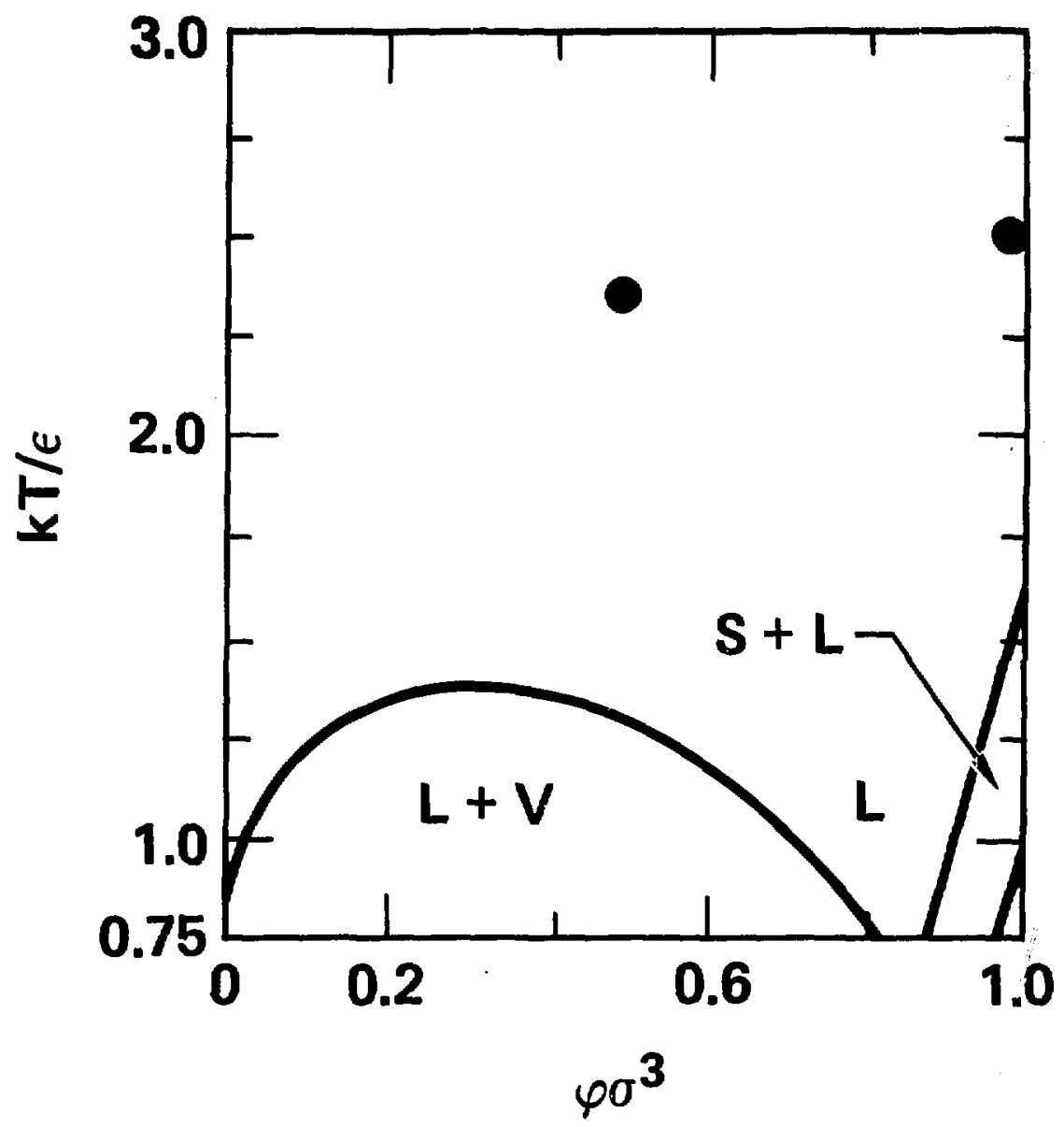


Fig. 7.31. Evolution of two pressurized cubes. The curves do not include monomers in either the energy or the population summations.
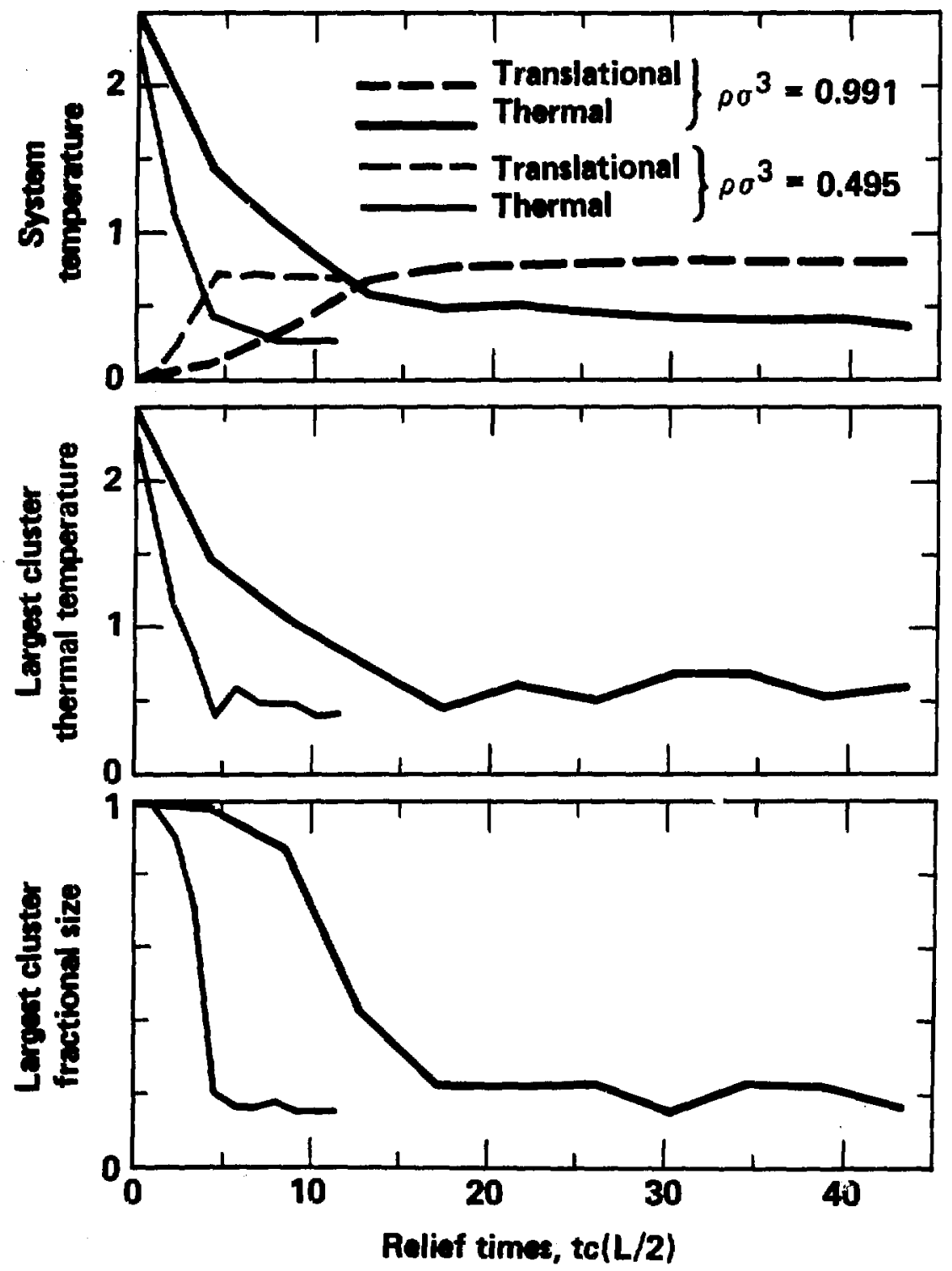
7.4. Comparison of the Molecular Dynemics Results to the Models Derived in Chapter Five

The hydrodynamic models, (1), (2), (3) and (4), from Chepter five predict $N_{C} \sim N^{0} P^{-2}, N^{0} P^{-4}, N^{2 / 3} P^{-4 / 3}$, and $N^{-2}$, respectively in two dimensions,. In three dimensions, the four nodels predict $N_{C} \sim N^{0} P^{-3}, N^{0} P^{-6}, N^{2 / 3} P^{-2}$, and $N P^{-3}$.

In thls section, the two-dimensional nolecular dynanic results (for the larger fragnents) are plotted on log-log graphs to datermine mich power law applies. The result is that modal 3 is the only modal consistent with the nolecular dynamics data. Then, quantitative predictions using model 3 are made for all the fragments with more than nine atoms (a size that eliminates an inconveniently large number of very small fragments); these predictions are a function of the observed fragment size because of the size dependence of the surface tension. The translational temperatures of the two-dimensional fragnents are also compared with the upper bound prediction of simple hydrodynamics. Finally, the two available three dimensional nolecular runs are compared to the predictions of model 3.

For a quant1tative comparison of the molecular dynamics results to model 3, it is convenient to plot as figures of merit, the ratios of the observed to the predicted fragment fractional size and translational temperature. These quantities are

observed

$$
\begin{array}{ll}
\frac{N_{C}}{N} & =\left[\frac{\pi}{N}(D+2)^{D}\left(\frac{D+1}{D}\right)^{D-2} \gamma_{p}^{D+1}\left(\frac{c}{p}\right)^{2 D}\right]^{1 / 3} \\
T_{t r}\left(=\frac{2}{D} \frac{K_{C}}{N_{c}}\right) & =\frac{1}{D}\left(\frac{P}{\rho C}\right)^{2}
\end{array}
$$$$
\text { predicted }
$$ 
where $N_{c}$ and $K_{c}$ are the population and translational energy of the observed cluster. The system population, $N$, does not include monomers In order to remove some of the influence of vapor pressure formation. When the ratio of observed to predicted fragment size or temperature is greater than one, an underprediction was made. It should be noted that the translational temperature prediction is an upper bound since It assume that all the kinetic energy produced by the relief wave is translational, actually, a portion of the kinetic energy is Initially dilational (and eventually thermal). For extensive fragmentation, Eq. 5.35 shows that model 3 predicts a reduction in the translational temperature of $\left[1-0.6\left(N_{c} / N\right)^{2 / 3}\right]$ in three dimensions and of $\left[1-0.5\left(N_{c} / N\right)\right]$ in two dimensions. Because the average observed quantitles were always above the upper bound, the upper bound prediction was used for simplicity unless otherwise noted.

The time of the observation influences the figures of merit. The observation time should be late enough that fragmentation is essentially complete, but as early as possible to minimize the influence of vapor pressure formation. Because the energy compliations were intermittent, only a limited set of observation cholces existed. The times selected are listed in Table 7.5. In cases where fragmentation was marginal, considerably more time (in rellef time units) elapsed before fragmentation. Examination of the atomic position plots for these cases indicates that some regions oscillated (changed overall shape) several times before fragmenting.

For convenlence, only fragments with ten or more atons were considored; smaller fragnents were too numerous and too easily 
Table 7.5. Times at which the fragment sizes and trenslational temperatures were compared to the predicted values.

Populationt

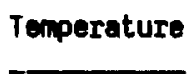

Reduced TIm

Iwo diansionel runs

14491

2611

2611

2611

721

721

721

721

721

169

169

169

169

250

250
1.01

1.16

0.76

0.65

1.17

0.77

0.65

0.55

0.51

1.19

0.77

0.62

0.60
104.3

38.4

76.9

71.4

29.4

70.3

74.4

78.0

80.0

9.1

10.8

74.7

81.0
Three dimensional runs

2.36

2.50

6.9

6.9
4.4

4.2

6.4

5.2

6.0

11.1

12.0

11.5

11.9

4.5

4.5

27.7

31.2

5.7

21.6

including mononers 
confused with the vapor. In evaluating Eq. 7.72 , the surface tension was computed for: the observed (rather than predicted) fragment size. Also, if the predicted fractional fragment size was larger than 1.0, It was reduced to 1.0. (Model 3 does not apply when there is no fragmentation.) This correction was only required for 22 of the 390 two dimensional fragnents, and for two of the eleven three dimensional fragments; the fragments needing correction were almost always from essentially non-fragnenting runs. Finally, three fragments coulo not be evaluated because the temperature was above this critical point. This furnishes a reminder that the temperatures read from the results are instantaneous values rather than proper time averages, which accounts for some of the scatter in the results. 
7.4.1. Comparison of the Two Dimensional Nolecular Dymenics Resits to the Hydrodynamic Models

The fragment sizes (In atoms) from two 2611 atom runs are shown in Fig. 7.32. The largest fragment, the average of the ten largost fragnents, and the average of the largest fragments comprising $30 \mathrm{w}$ of the non-monomer population are shown. In eesh case, the raw data have been slightly adjusted (using the energy minimization model, "model 3") to unlform initial conditions (26.1 non-monomer atoms, $p=0.6, c=4.0$, and $\gamma=0.32$ ). The only remining varlable in modal 3 is the initial pressure. The slopes of the three fragnent size curves are $-1.03,-0.86$, and -1.42 , respectively; these values bracket the predicted slope of $-4 / 3$ from model 3 reasonably well. To lllustrate the sensitivity of the fragmentation to fragment-slze dependent surface tension, the prediction curve is also shown using the lower suy "ace tensions at the artual fragment slzes; the "observed" curves would be similarly steeper when the surface tension correction (to $Y=0.32$ ) is eliminated. Finally, the other model predictions are shown. The slopes of models 1,2 , and $4(-2,-4$, and -2 , respectively) are much steeper than the molecular dynamics results.

The fragment sizes from the highest pressure run for each system size (14491, 2611, 721, and 169 atons) are shown in Fig. 7.33. The nomenclature is identical to the previous figure. As in Fig. 7.32, the data were slightly adjusted to uniform initial conditions $(P=1.4, \rho=0.6, c=4.0$, and $\gamma=0.32)$. The only remaining variable In the models is the system size (total number of particles), $N$. The slopes of the middle section of the thiee fregment size curves are $0.75,0.69$, and 0.64 , respectively. These observed values bracket the 
Flg. 7.32. Observed and predicted fragnent sizes (atoms) as a function of initial pressure, for two 2611 atom runs. The data and model predictions have been slightly adjusted to constant initial conditlons. The three observed values for each run are for the largest fragment, the average of the ten largest fragments, and the average of the largest fragments comprising $30 \%$ of the system population (less monomers).

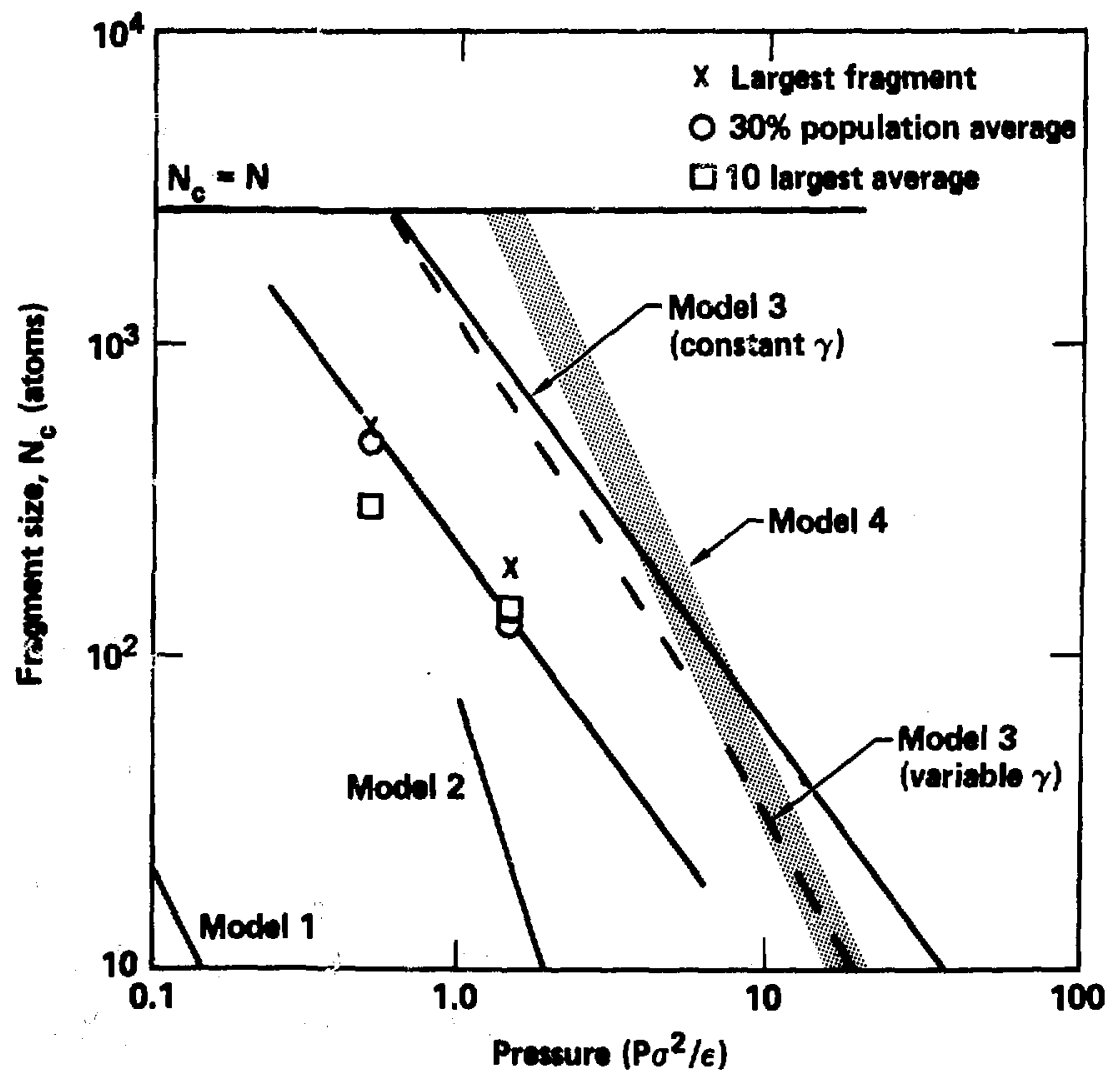


Fig. 7.33. Observed and predicted fragment sizes (atoms) as a function of system size, for simllar heating levels. The nomenclature is identical to Fig. 7.32.

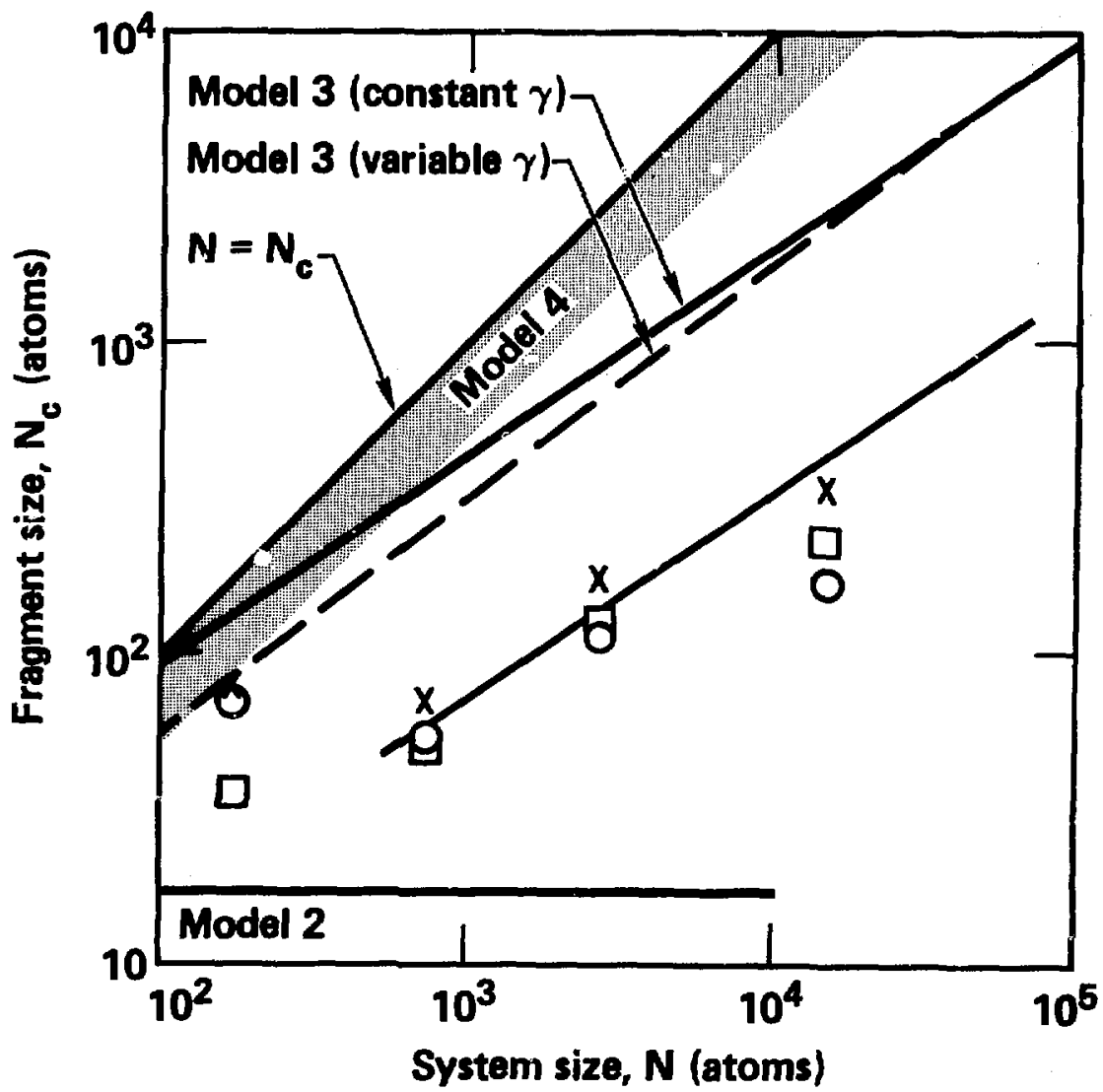


predicted slope of $2 / 3$ from modal 3 . The 169 atom data were not used to compute the slope because of minimal fregmentation. The 14491 atom fragment sizes are probably a bit low. Position snapshots and density profiles indicate that a sloshing motion was present in the system at the tim the confining boundary was removed. However, if the slopes are computed using line segrents between the 721 and 14491 data, the results are $0.54,0.50$, and $0 .\{1$, respectively. Thus, the mo?ecular dynanics results indicate a possible system size dependence of the 2/3 power (the 11lustrated line), but support that slope with an uncertainty of \pm 0.15 . This result ggain is consistent with the energy minimization model (model 3). Modeis 1 and 2 include no system size dependence akd aci Jidingly predict very low fragnent sizes for this initlal conditinn ( 0.1 and 18 atons, respectively). Model 4 predicts a much steeper slope (1.0) than found in the molecular dynemics runs.

Although the data in Figs. 7.32 and 7,33 support the energy minimization model's power law dependence of fragment size on system size and isochorlc hetiting level (initlal pressure), the magnitudes of the observed sizes (in atoms) are about ten times lower than the model 3 prediction. It is therefore suggested that the predictions of that model be reduced tenfold in applications. Macroscoplc systems require extensive extrapolation; a $10 \mathrm{~cm}$ radius HYLIFE jet cross section contains about $2 \times 10^{17}$ 11thium atoms (13 more orders of magnltude in system population). If the 14491 atom run initial conditions (tomperature and density) are applied to $2 \times 10^{17}$ atom system with 1ithium values of o and $c$ (Table 4.6), the predicted fragment size is $2 \times 10^{12}$ atoms $\left(10^{-5}\right.$ fractional size), or $0.31 \mathrm{~mm}$ radius 
C0.10 $\mathrm{mm}$ radius if the factor of 10 raduction in predicted freoment popuiation is epplied). The heating level in that run was $10^{-20}$ J/bond, about seven times the maximum level expected in HrLIFE. The fragment radius is about $1 / 7$ th the 3-D fregment redius predicted using known lithlum properties (Section 5.3.3). Beceuse the extrepolation In gystem size is so large, macroscopic experlments are assential. Howover, the reasonable agrewment batween the nodel and microscopic (computer) experiments allows use of the model to desion the experiments.

The preceding discussion of the average observed date is extendad below to include individual predictions for each fragunt containing ten or more atoms. The extended enalysis uses surface tension evaluations at the observed fragment size to make the predictions. The prediction accuracy is exemined to determine its dependence on the fragment size, and the sensitivity to orrors in either the sound speed or the surface tension is discussed.

The fragment size results for all fragments with ten or more atoms in all the two dinensional runs are shown in Fig. 7.34 as a function of the temperature following lsochoric heating. The observed sizes were usually sonewhat smaller than the prediction. The largest fragment data point from each run is indlcated by a bold symbol; these . fragnent sizes were closer to the prediction then the smaller fragments (Table 7.6 ).

The fragment size floure of merit results are cross plotted as a function of observed fragment size in Figs. 7.35 and 7.36 for the three small systems and the 14491 system, respectively. There was a definite fragment size dependence with the largar fragments being 
Fig. 7.34. Ratio of the observed to the predicted fragnent sizes (atoms) for all fragments containing ten or more atoms in the thirteen 2-D molecular dynamics runs. The prediction is from nodel 3 (Eq. 7.72).

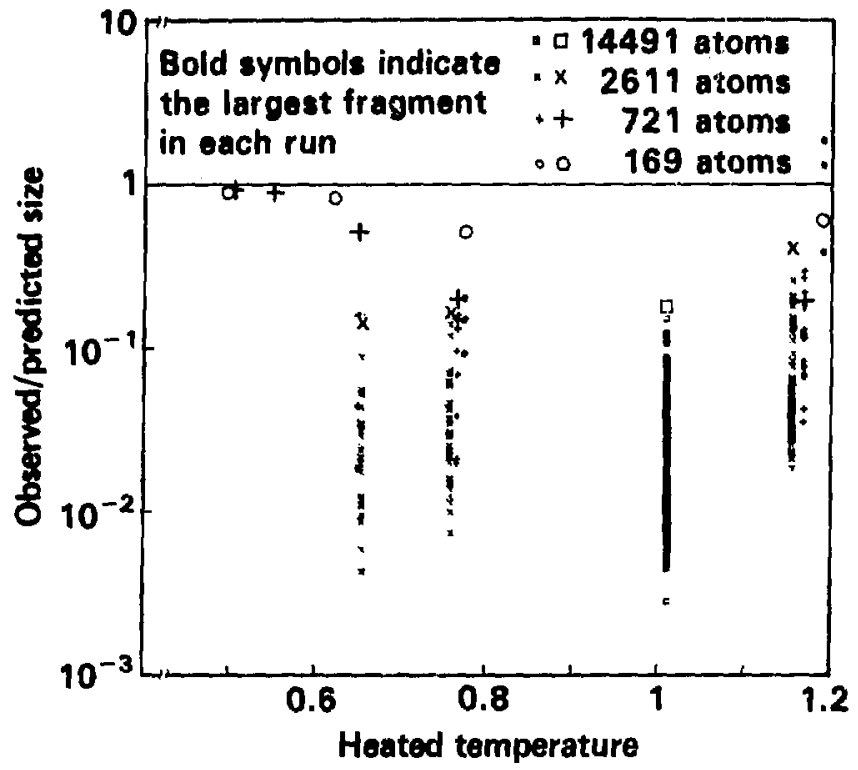


Table 7.6. Observed and predicted fragment fractlonal sizes (atcoss) for the largest fragnent in each 2-D molecular dynemics run. As before, $N_{c}$ indicates the cluster population, $N_{m}$ is the numbrer of monomers, and $N$ is the original system population.

Nt Temperature Observed After

- Hating

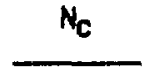

Ne $/\left(\mathrm{N}-\mathrm{N}_{\mathrm{m}}\right)$

Observed Pradicted Predlcted Predicted

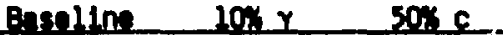

$\begin{array}{rrrllll}14491 & 1.01 & 375 & 0.028 & 0.74 & 0.051 & 0.094 \\ 2611 & 1.16 & 136 & 0.056 & 0.14 & 0.030 & 0.056 \\ 2611 & 0.76 & 270 & 0.11 & 0.68 & 0.15 & 0.27 \\ 2611 & 0.65 & 361 & 0.15 & 1.001^{*} & 0.22 & 0.40 \\ 721 & 1.17 & 44 & 0.069 & 0.35 & 0.76 & 0.14 \\ 721 & 0.77 & 117 & 0.18 & 0.91 & 0.20 & 0.36 \\ 721 & 0.65 & 337 & 0.52 & 1.5^{*} & 0.33 & 0.61 \\ 721 & 0.55 & 593 & 0.90 & 4.1^{*} & 0.50 & 1.6^{*} \\ 721 & 0.51 & 626 & 0.94 & 16.6^{*} & 3.6^{*} & 6.6^{*} \\ 169 & 1.15 & 41 & 0.27 & 0.42 & 0.09 & 0.17 \\ 169 & 0.77 & 64 & 0.43 & 0.82 & 0.17 & 0.31 \\ 169 & 0.62 & 129 & 0.85 & 2.46^{*} & 0.53 & 0.98 \\ 169 & 0.50 & 142 & 0.92 & 23.8^{*} & 5.1^{*} & 9.4^{*}\end{array}$

t Including monomers

* adjusted to 1.0 for the graphs 
Fig. 7.35. The ratio of the observed to the predicted fragment size (atoms), plotted as a function of the observed size, for twelve 2-D molecular dynalcs fragmentation ruris. The smooth curve is the locus of predicted sizes equal to the original size; the predictions have been adjusted in a faw cases to prevent predictions exceeding the original size.

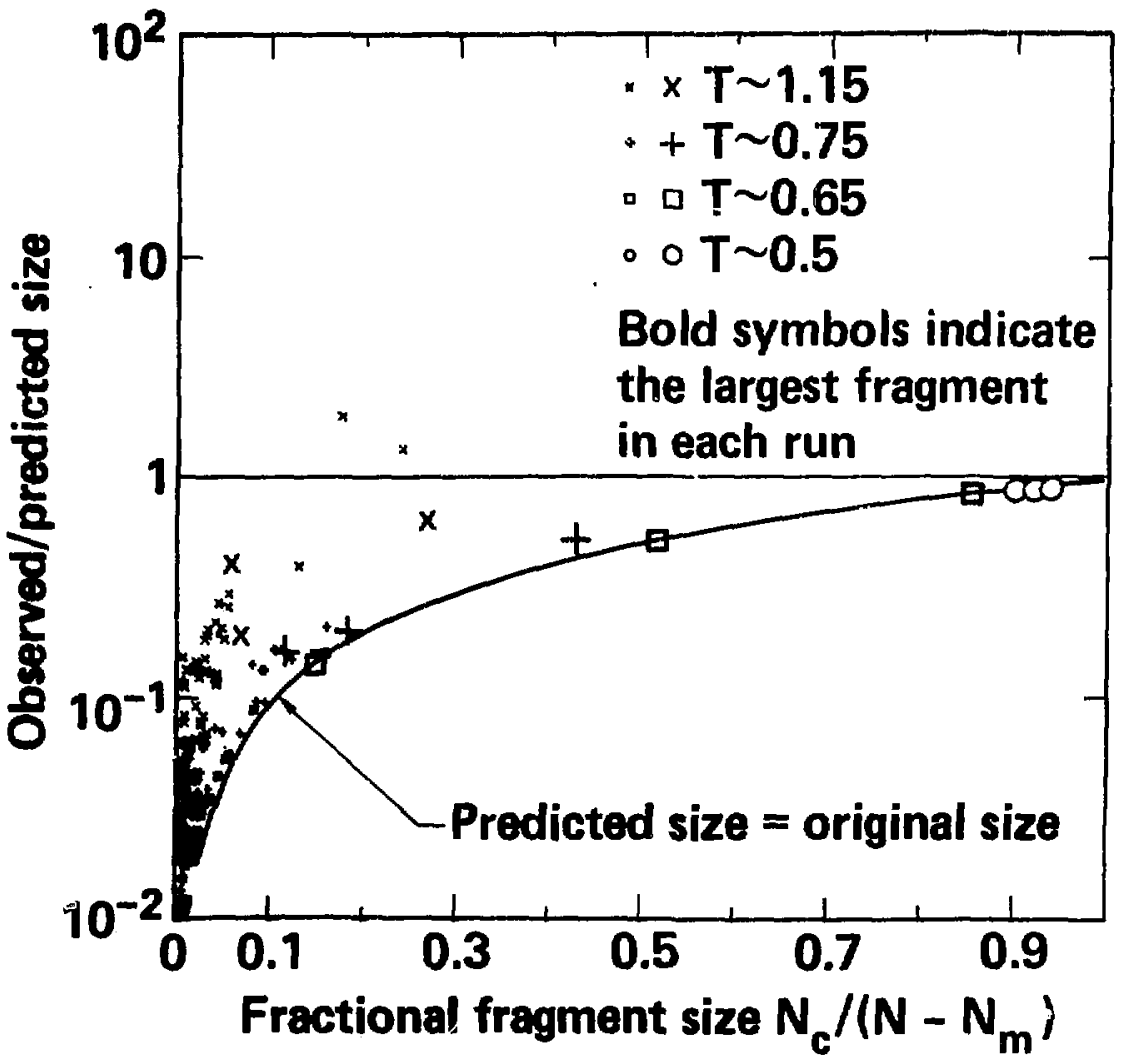


F1g. 7.36. The ratio of the observed to the predicted fragment size (atoms), plotted as a function of the observed size, for the 14491 atom molecular dynamics fragmentation run.

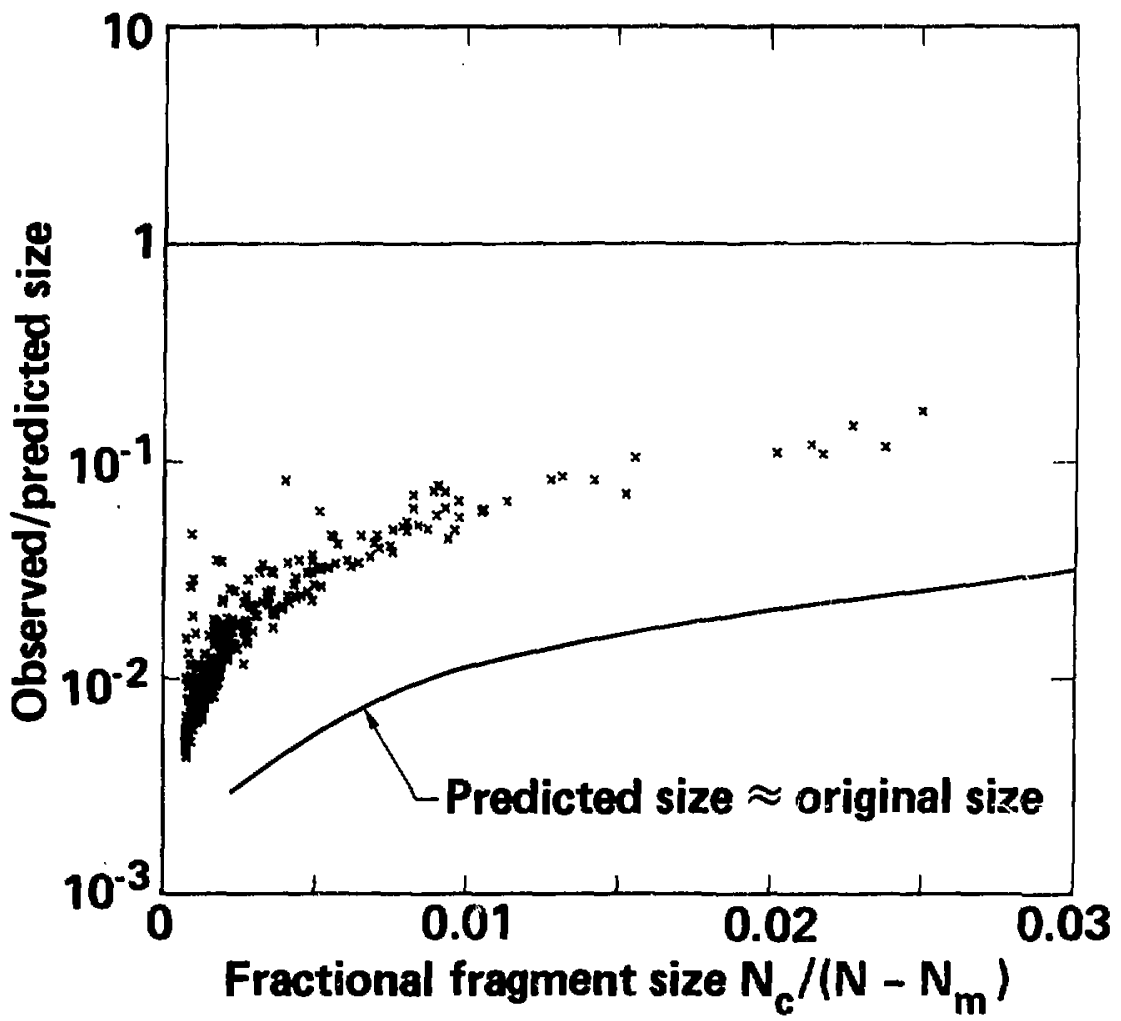


predicted the nost accorately. Flgure 7.35 is deceptive, however, because the five largest data points plotted were predicted to remain Intact by varying margins (Table 7.6). If the non-physical predictions of fragments larger than $100 x$ of the system size were used for these points, some of them would be considerably above the ratio $=1.0$ line. Navertheless, the larger fragments of the vigorously fragmenting systems were predicted quite woll (a factor of 1.5 to 6 larger than the observed values).

The smaller fragnents were not predicted very well by the model even though the surface tension was evaluated at the observed fragment size. Possible explanations for this result include the following:

- The small fragnents were remnants from reglons between larger fragments, and thus did not meet the energy balance criterion of model 3 .

- The large local energy fluctuations in these small systens were conducive to loss of snall fragments.

- The surface tension property was grossly incorrect; either due to an error in the equilibrium curves, or due to use of Instantaneous temperatures rather than time averages to specify 1t. (This possib!'ity was investigated by replotting Flgs. 7.35 and 7.36 using values of the surface tension reduced by an order of manitude and also by using values of the sound speed reduced by a factor of two. Although this drastic "adjustment" of properties improved the average behavior, it did not remove the characteristic fragment size dependence of the observed-to-predicted ratio; i.e. the shape of the data did not 
change.)

- Vepor pressure formation was responsible for the production of small fragments (even those with more then ten atons).

- The higher velocity divergence near the original systen center would reduce the prediction by a factor of $>(0.2)^{-2}=25$. in that region (Figs. 5.5 and 5.6), (But the total mass of small fragments was too large to be explained solely by this argument.)

At fragmentation, the fragments are definitely non-circular (e.g. Fig. 7.21). Thus, the surface to volume ratlo will be higher than for a sphere (or circle), and one might speculate that the effective (instantaneous) surface tension will be higher than the equilibrium value. However, in an equilioriun drop, the instantaneous configuration is almost always non-circular, so that the "equilibrium" surface tension value includes the additional (time-averaged) surface area. Thus, the non-circular Initial fragment shape probably does not force the production of a higher surface tension (and consequently, fragnent size).

The ratios of the observ. \& cluster translational temperatures to the prediction from simple planar hydrodynamics are plotted in Figs. 7.37 to 7.39 , which are analagous to Figs. 7.34 to 7.36 . As Fig. 7.37 shows, the fragment translational temperatures were distributed on both the high and low sides of the predictions.

The translational temperature mean appears to be at about the sams level above the prediction for each fragment size (Figs. 7.38 and 7.39), 1.e. fragment size was not correlated with fragment 
F1g. 7.37. Ratio of the observed to the predicted fragment trenslational temperatures (Eq. 7.73) for all fragments containing ten or more atoms in the thirteen 2-D nolecular dynenics runs.

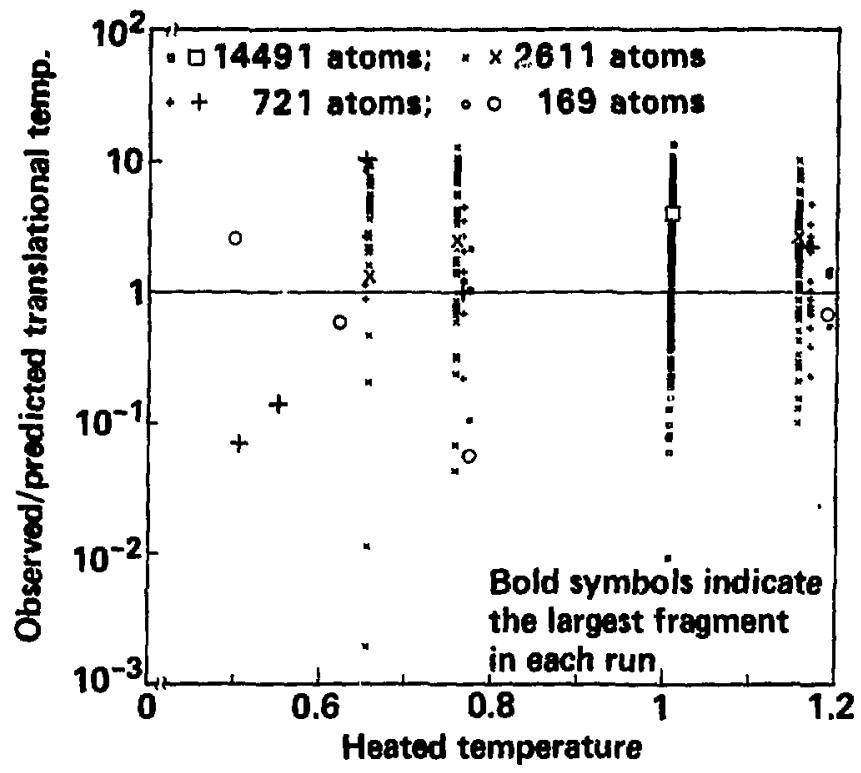


Fig. 7.38. The ratio of the observed to the predicted fregment translational temperatures, plotted as a punction of the observed size, for twelve 2-D molecular dynamics fresmentation runs.

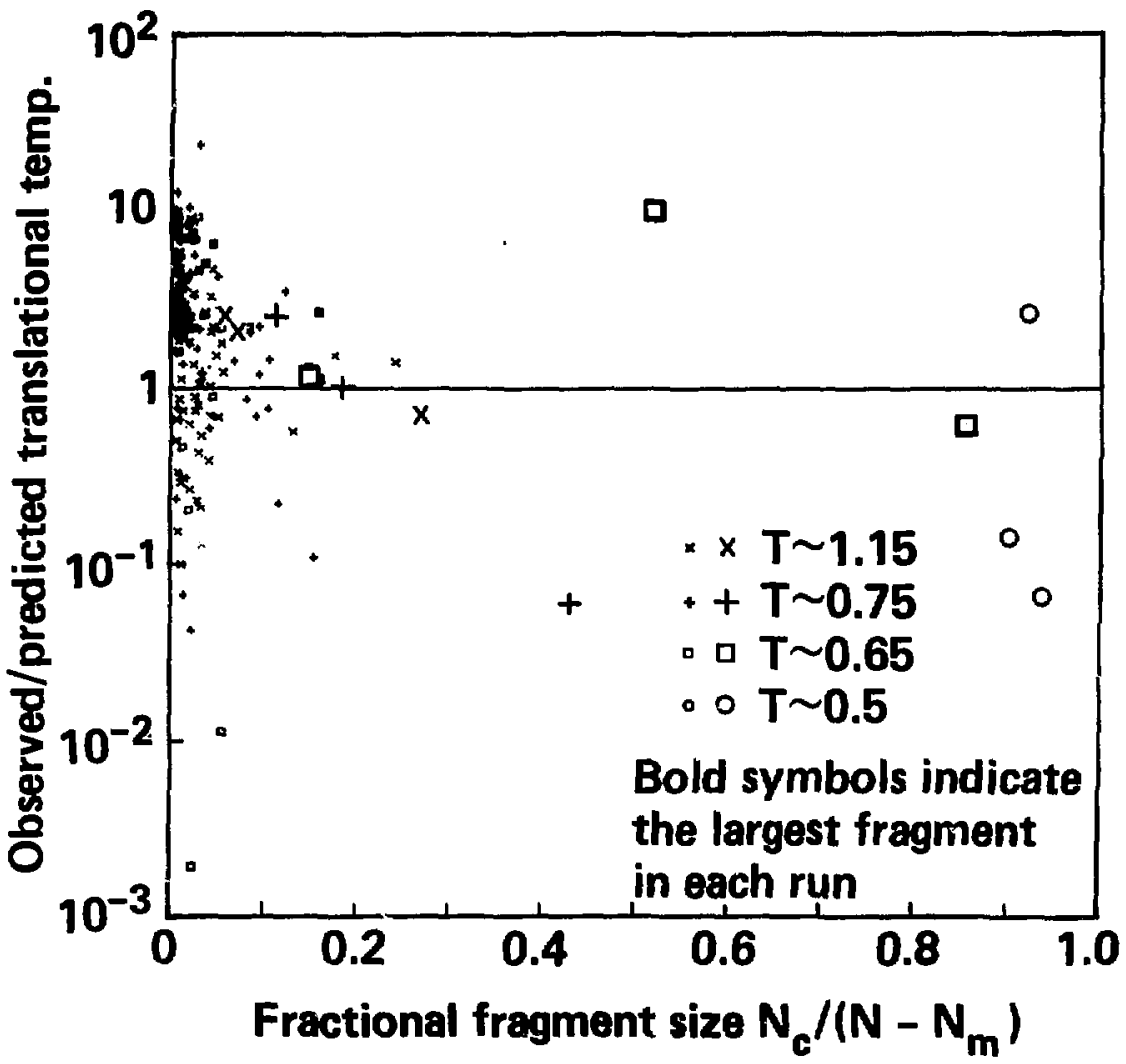


Fig. 7.39. The ratio of the observed to the predicted fragment translational tomperatures, plotted as a function of the ooserved size, for the 14491 atom molecular dynemics fragmentation run.

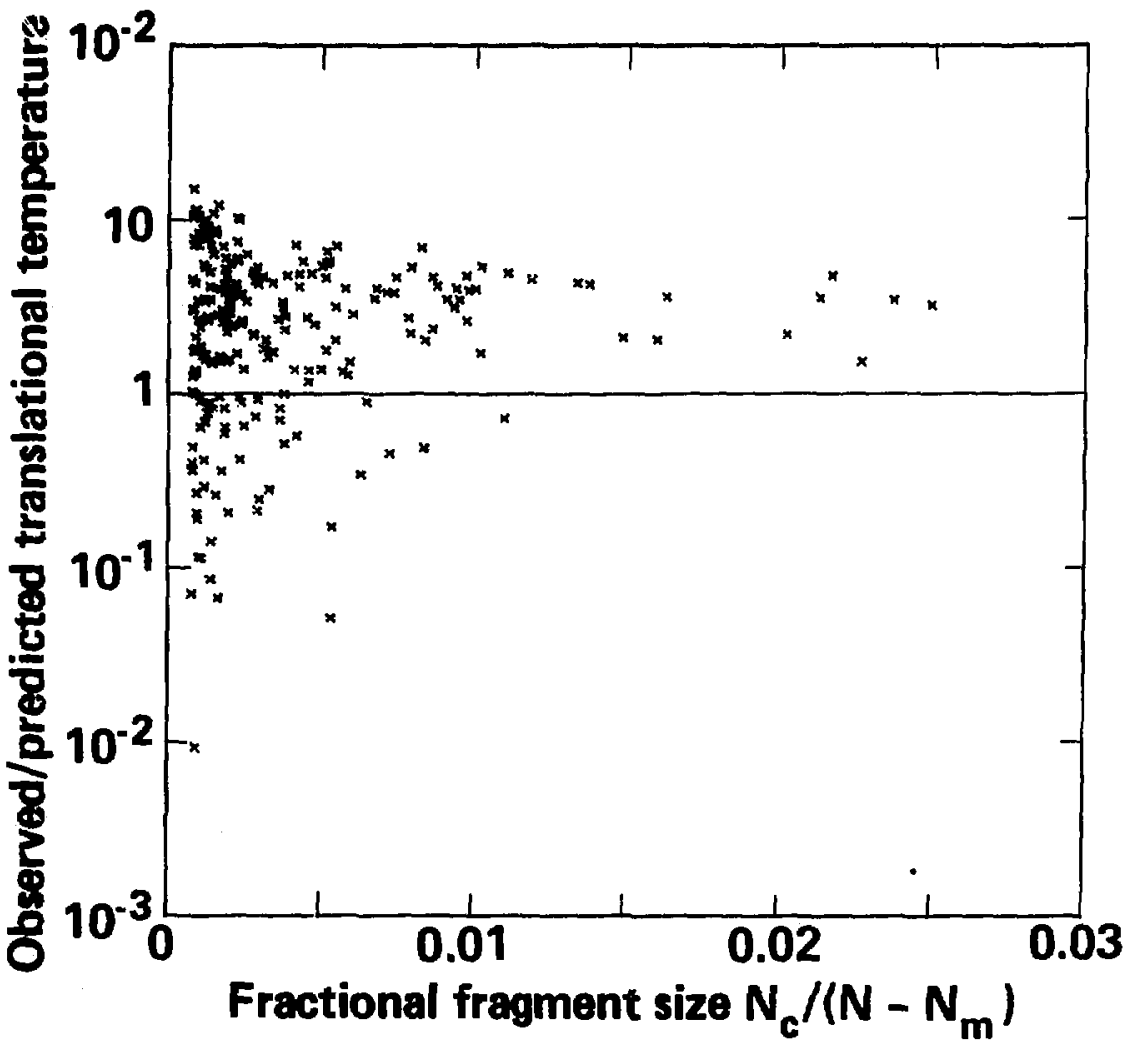


translational energy. However, the smaller fragments showed a wider temperature distribution than the large fragments. This is reasonable; if a system breaks into only a fow large equal-sized fragnents, those fragnents must have roughly equal momenta (to not alter the initial zero momentum). Conversely, if there are a large number of small fragments, many possible comblnations of wide-ranging momenta can satisfy monentum conservation. Flnally, beth Figs. 7.38 and 7.39 show fragments with very low energy, but no fragments with very high energy. This is also reasonable; a fragment that only marginally escapes could have almost no translational energy. Conversely, because there is only a limited anount of energy in the system, there 1s an upper bound on the fragment translatlonal enerny, and concentrating most of it in only one fragment is unlikely.

Because the surface tension does not enter into the fragment translational temperature prediction (Eq. 7.73), there is no sensitivity of the temperature figure of merit to a change in surface tension. The sensitivities of the translational temperature distributions to a $50 x$ decrease in sound speed were examined by repeating the plots with the lower values of sound speed. For the 14491 atom run, the decreased sound speed improved the results, although a somewhat smaller decrease would have been better. For the other runs, decreasing the sound speed degraded the results.

The overall translational temperature observations and predictings (ignoring clusters with less than ten atoms) are listed in Table 7.7. The translational temperatures (lgnoring morimer energy and mass) are shown in Fig. 7.40 as a function of system size and Initlal pressure. The inset shows the observed translational temperatures wich properly 
Table 7.7. Observed and predicted fragment translational temperatures for the largest fragment in each 2-D molecular dynamics run. As before, $N_{c}$ and $K_{c}$ refer to the population and translational energy of one cluster. The "Observed $K / N^{n}$ is the overall translational energy per atom (ignoring monomer energy and population). The "Predicted $\mathrm{K} / \mathrm{N}^{*}$ is the translational temperature (translational energy per atom) predicted by Eq. 7.73 wich is independent of population.

\begin{tabular}{|c|c|c|c|c|c|}
\hline $\begin{array}{l}N^{\dagger} T \\
--\end{array}$ & $\begin{array}{c}\text { Temperature } \\
\text { After } \\
\text { Heat ing } \\
\end{array}$ & $\begin{array}{c}\text { Observed } \\
\mathrm{K} / \mathrm{N} \\
\text { total } \\
\end{array}$ & $\begin{array}{l}\text { Observed } \\
K_{c} / N_{c}+t \\
\text { largest } \\
\end{array}$ & $\begin{array}{l}\text { Predict.ed } \\
\text { Baseline }\end{array}$ & $\begin{array}{c}N \\
\text { Predicted } \\
50 \% \mathrm{C} \\
\end{array}$ \\
\hline 14491 & 1.01 & 0.35 & 0.33 & 0.10 & 0.41 \\
\hline $\begin{array}{l}2611 \\
2611 \\
2611\end{array}$ & $\begin{array}{l}1.16 \\
0.76 \\
0.65\end{array}$ & $\begin{array}{l}0.77 \\
0.15 \\
0.072\end{array}$ & $\begin{array}{l}0.38 \\
0.099 \\
0.021\end{array}$ & $\begin{array}{l}0.15 \\
0.034 \\
0.017\end{array}$ & $\begin{array}{l}0.59 \\
0.16 \\
0.068\end{array}$ \\
\hline $\begin{array}{l}721 \\
721 \\
721 \\
721 \\
721\end{array}$ & $\begin{array}{l}1.17 \\
0.77 \\
0.65 \\
0.55 \\
0.51\end{array}$ & $\begin{array}{l}0.36 \\
0.10 \\
0.055 \\
0.029 \\
0.018\end{array}$ & $\begin{array}{l}0.30 \\
0.043 \\
0.19 \\
0.00052 \\
0.00004\end{array}$ & $\begin{array}{l}0.14 \\
0.043 \\
0.019 \\
0.0037 \\
0.00058\end{array}$ & $\begin{array}{l}0.58 \\
0.17 \\
0.077 \\
0.015 \\
0.002\end{array}$ \\
\hline $\begin{array}{l}169 \\
169 \\
169 \\
169\end{array}$ & $\begin{array}{l}1.19 \\
0.77 \\
0.62 \\
0.50\end{array}$ & $\begin{array}{l}0.34 \\
0.067 \\
0.043 \\
0.022\end{array}$ & $\begin{array}{l}0.12 \\
0.003 \\
0.009 \\
0.002\end{array}$ & $\begin{array}{l}0.17 \\
0.051 \\
0.015 \\
0.0008\end{array}$ & $\begin{array}{l}0.68 \\
0.20 \\
0.061 \\
0.003\end{array}$ \\
\hline
\end{tabular}

t including monomers

tt for the argest cluster only 
Fig. 7.40. Translational temperatures (scaled to the prediction of model 3) for the 13 molecular dynemics rins.

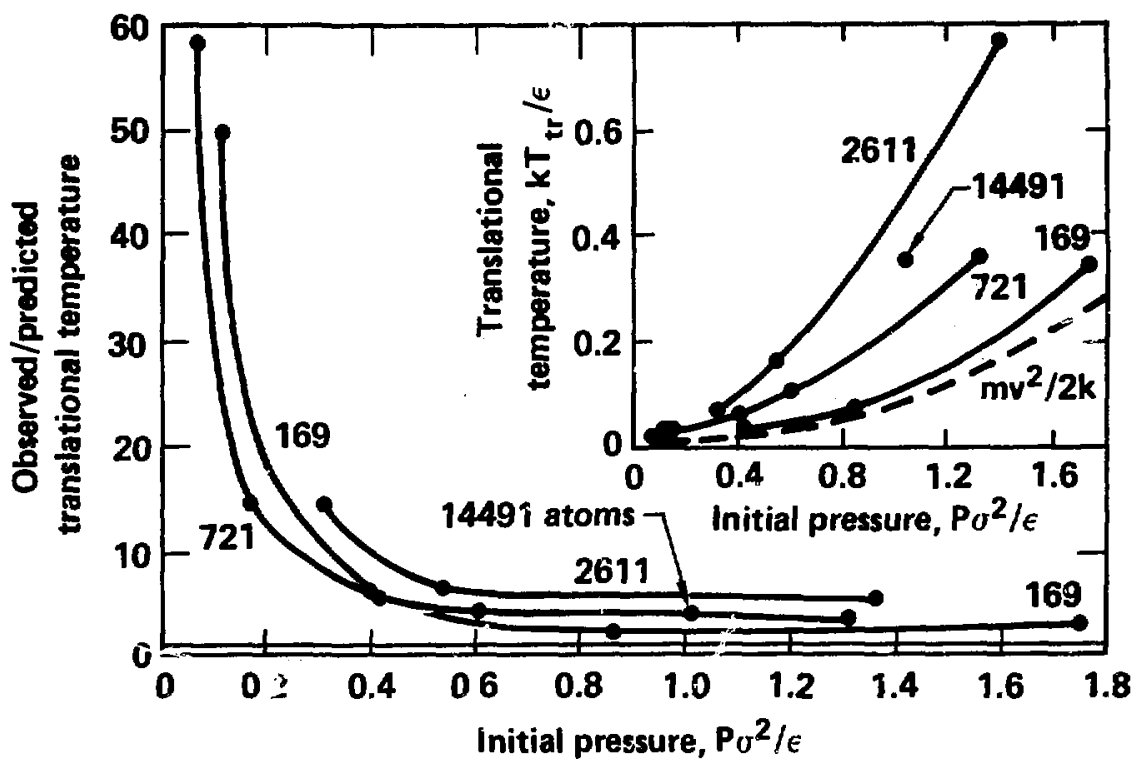


Increase with heating level (initial pressure). All the values exceed $v^{2} / 2$, where $v$ is the jump-off velocity, (dashed line). In two dimerisions, model 3 predicts

$$
T_{t r}=\left(v^{2} / 2\right)\left[1-\frac{1}{2} \frac{N_{c}}{N}\right]
$$

For extrems fragmentation $\left(N_{c} \rightarrow 0\right)$, Eq. 7.74 produces the correct upper bound, but for no fragmentation Eq. 7.74 predicts $1 / 2$ the upper bound, rather than zero. The fallure at no-fragmentation is a consequance of the assumption of a homogeneous expansion in model 3 .

The maln portion of the flgure shows the ratlo of the observed to the predicted (Eq. 7.74) translational temperatures. The additional kinetic energy is from two sources: vapor pressure formation (which is Iimited in Fig. 7.40 by the elimination of monomers from the calculation), and fragnentation due to fluctuations (a phenomenon observed for small systens on the saturation line with no l.sochoric hasting).

For macroscopic systems, model 3 suggests that both vapor pressure formation and fracture due to "equilibrium" fluctuations are negliglble. Glen's three dimensional (macroscopic) hydrodynemic code calculations ${ }^{46}$ confirmed the upper bound of $v^{2} / 2 k$. The molecular dynemics calculations (Flg. 7.40) show that the discrepancy tends to Increase with system slze, at least for systems of a few thousand atcos or less. However, the 14491 atcm result shows a decreased discrepancy (oven though its Initlal sloshing motion would be expected to produce more trenslational energy then en initially quiescent systam). Thus, these linited molecular dynamics results are not 
inconsistent with model 3. Finally, note that the peak in the excess trenslational temperature is in the region of tha critical nuclous size. Systems smaller than the critical size are naturally unsteble, leading to fragentation oven in saturated systems. 
7.4.2. Comparison of the Three Dimensional Molecular Dynemics Results to Model 3

The ratios of the observed to the predicted sizes are shom in Fig. 7.41. The high pressure $\operatorname{run}(P=17.9, T=2.5, p=0.99)$ was predicted very wall by the model (to within a factor of two). The lower pressure $\operatorname{run}(P=1.5, T=2.4, p=0.5)$ was overpredicted by the model (by factors of 5 to 13). The cluster sizes and temperatures for both runs are listed in Table 7.6.

The ratios of the observed to the predicted translational temperatures are shown in FIg. 7.42. Here the situation was reversed; the high pressure run was overpredicted by factors of 16 to 330 wille the low pressure run was predicted very well. The translational temperature date are also listed in Table 7.8.

The high pressure run had a predicted translational temperature of 1.1. The observed values for the large clusters were much lower (s 0.1 ); however, the non-monomer translational temperature was 0.78 whlle the overall trenslational temperature was 0.83 . Thus, in this case, the fragment sizes were properly predicted, but the fragment translational energy was concentrated in the small fragments.

The low pressure run had a predicted translational temperature of 0.20. The four largest fragments had translational temperatures ranging from 0.018 to 0.48 . The non-monomer translatlonal temperature was 0.70 wile the overall translational temperature was 0.00 . Thus, vepor pressure formation doninated thls case. The higher number of monomers in this run was fu. ..... idence that vepor pressure was cominent. 
F1g. 7.41. The ratio of the observed to the predicted fregment size (atcons), plotted as a function of the observed size, for the two 3-D molecular oynemics fregmentation runs.

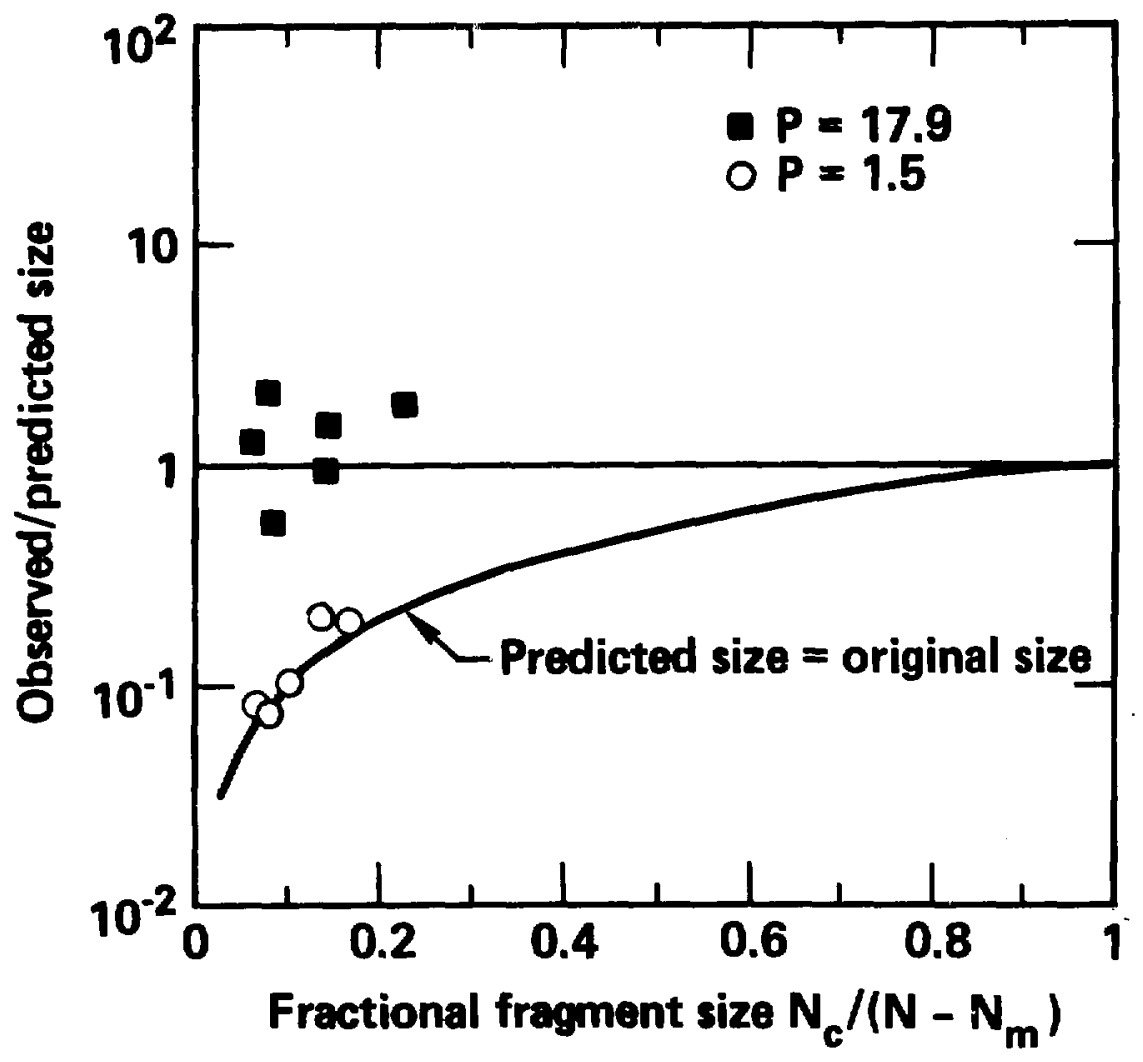


Table 7.8. Observed and predicted fragment sizes and translational temperatures for the largest fragments in the 3-D molecular dynamics runs. The varlables are defined as before; $N, N_{c}$, and $N_{m}$ indicate system, cluster, and monomer populations whlle Eq. 7.73 defines the trenslational temperature.

Fragunt Temperature

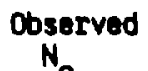

$$
N_{c} /\left(N-N_{m}\right)
$$
Observed Predicted

Translationsl Temp. Observed Predicted

$$
e=0.495, I=2.36, P=1.5
$$

0.59

0.60

D.32

0.34

0.43
30

25

18

14

12
0.17

0.14

0.10

0.078

0.067

0.82

0.68

$1.6^{*}$

$1.2^{\star}$

0.81

0.24

0.36

0.18

0.48

0.014

0.28

0.28

0.28

0.28

0. 28

$$
e=0.991, T=2.50, P=17.9
$$

$\begin{array}{llllll}0.62 & 46 & 0.23 & 0.13 & 0.0033 & 1.11 \\ 0.66 & 29 & 0.14 & 0.10 & 0.02 & 1.11 \\ 0.57 & 28 & 0.14 & 0.14 & 0.011 & 1.11 \\ 0.41 & 17 & 0.083 & 0.14 & 0.051 & 1.11 \\ 0.76 & 16 & 0.078 & 0.057 & 0.039 & 1.11 \\ 0.67 & 11 & 0.054 & 0.063 & 0.067 & 1.11\end{array}$

* Adjusted to 1.0 for the graph. 
Fig. 7.42. The ratio of the observed to the predicted fragment translational temperatures, plotted as a function of the observed size, for two 3-D molecular dynemics fragmentation runs.

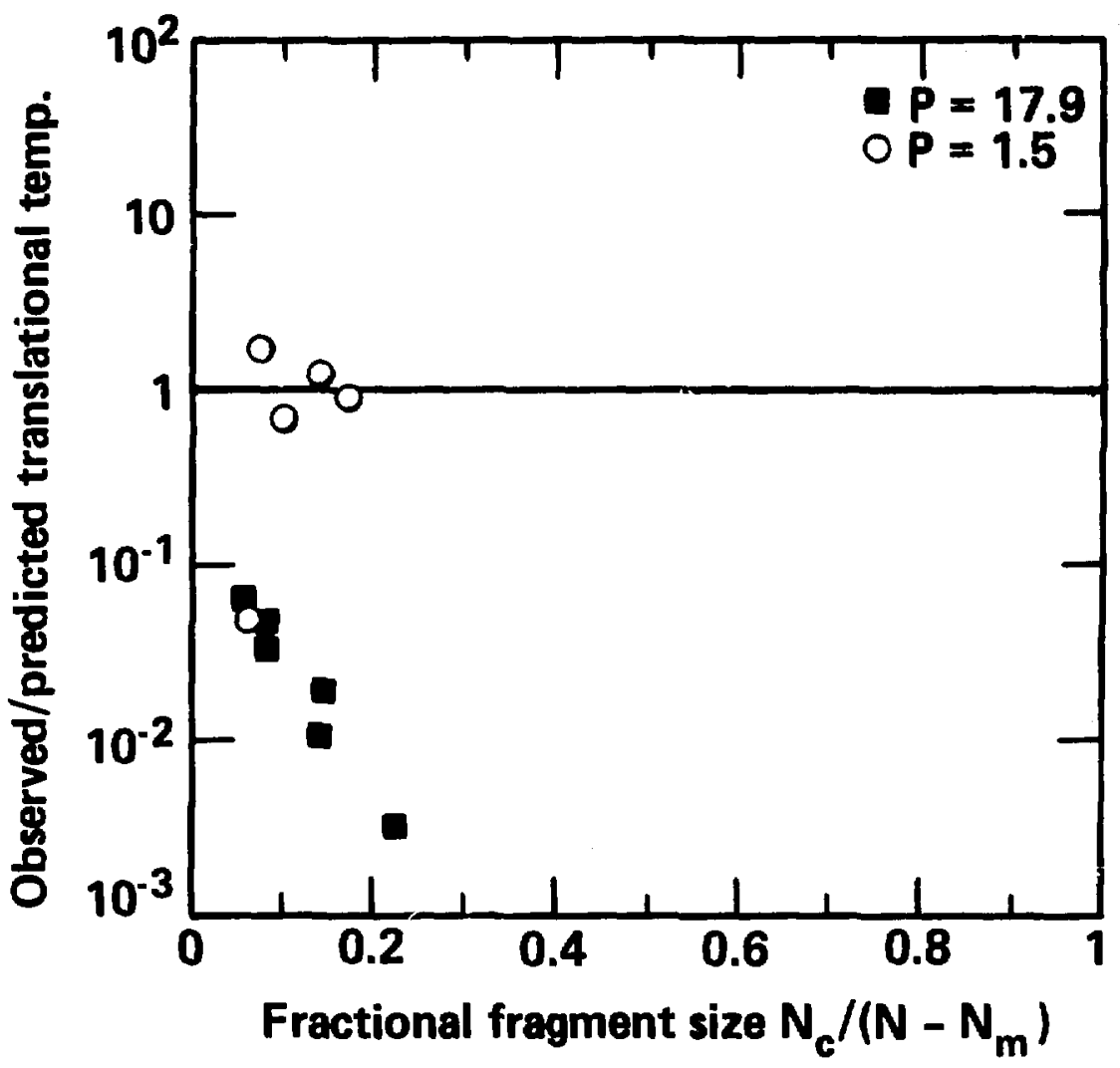




\subsection{Vapor Pressure Formation as a Competing Phenomenon}

then the boundaries were released, small clusters were ejected from the surface almost immediately due to the lack of an equilibrium surface and due to the high liquid temperature. In a normal sltuation, these lost atoms would be replaced by vapor atoms colliding with the surface. But, in the simulation, there were no reflecting boundarles to confine (and return) the vapor atoms.

Each atom that vaporized removed $\sim 1.9 c$ of potential energy from the llquid in addition to Its kinetic energy (typically 0.6 to 1.3e per monomer). To conserve energy, the kinetic energy of the bulk liquid decreased to offset the increased potentlal energy. Consider an inltial system with $N$ liquid atoms at $-1.9 \varepsilon$ potential energy per atom and temperature, $T$. After some time, there are $N_{m}$ mowomers with a measurable kinetic energy, $K$, (and no potentlal energy). The remaining liquid has $N-N_{m}$ atoms with -1.9e potential energy per atom and temperature, $T_{1}$. Assuming no change in density, the energy conservation equation is

$$
\begin{aligned}
& -1.9 N+N T=-1.9\left(N-N_{m}\right)+K+\left(N-N_{m}\right) T_{1} \\
& \rightarrow \Delta T_{1}=T-T_{1}=\frac{1.9 N_{m}+K}{N-N_{m}}-\frac{N m}{N-N_{m}} T .
\end{aligned}
$$

The temporal evolution of the monomers is shown in Fig. 7.43 for the 14491 atom run. The liquid cooling rate was remarkably constant. It would be expected to increase during fragmentation due to the Increasing surface area, and to decrease due to the decreasing liquid temperature. 
Fig. 7.43. Monomer population increase and resulting liquid cooling as a function of time for the 14491 atom run.

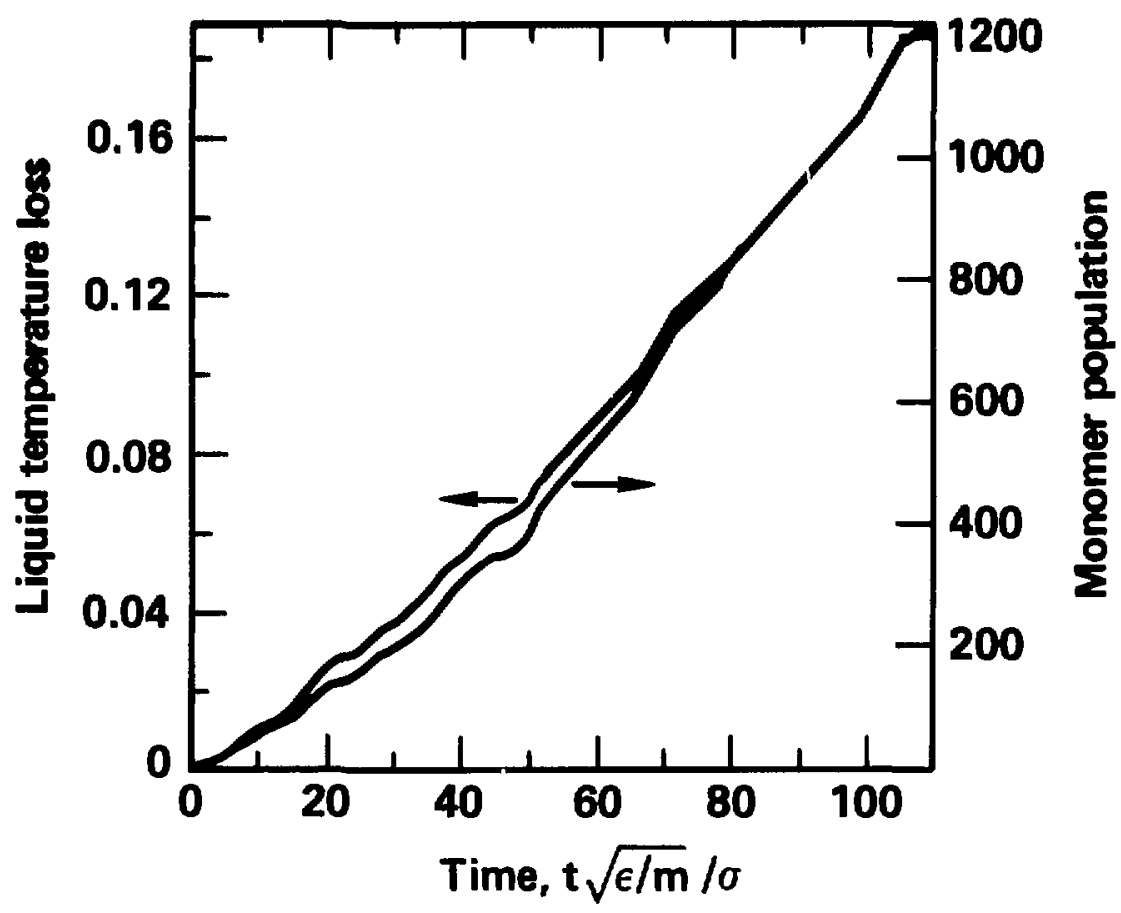


In Table 7.9, the monomer data from the 13 two dimensional molecular dynamics runs are shown. The times selected for the data collection are identical to the times used to evaluate fragmentation In Section 7.4.1. The last colum shows the predicted liquid temperature loss based on Eq. 7.75. When the isochorlc heating produced less than $\sim 0.2$ temperature Increase, vapor pressure formation renoved the added energy on the same time scale as the fragientation. Thus, only the two dimensional simulations with T 0.8 should be used to evaluate fragmentation

The mononer temperatures at the time of fragmentation for each run are listed in Table 7.9. The average liquid temperature during the monomer formation period lies somewhere between the peak listed in the second column of the table and the saturation temperature $(\sim 0.5)$. The monomer temperature exceeds both the average and the peak liquid temperatures in every case. Simflar results have been found by Vicentinl, Jacuccl and Pandharipande ${ }^{63}$ using three dimensional molecular dynemics for systems of 250 atoms. 
Table 7.9. Monomer population and energy for the 13 two dimensional molecular dynemlcs runs. The " $E / N_{m}$ " column includes both increased pntential energy and monomer kinetic energy.

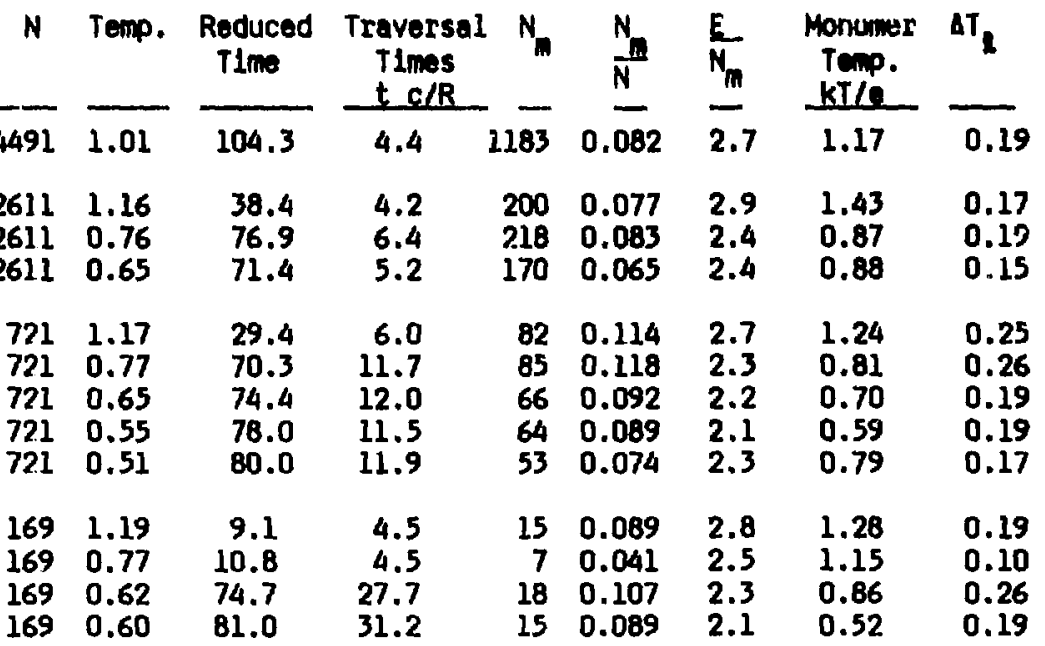


Chaplur Elght: Conclusions and Recommendations for Further Research

Fragmentation of free liquids in ICF reactor chembers could be the dominating factor in determining the upper bound on reactor pulse rate, Batwen pulses, the $x$-ray ablated materlals must cool and recuidense to allow oriver baen propagation for the next pulse. The Increased surface area caused by fragmentation w1ll enhance the cooling and condensation rates.

Atomization of liquid fuels in Internal combustion englnes could be an analogous process to isochorlc-heating-induced-fragmentation. Howaver, atomization theory is largely enplrical, and the differences In geometry make it hard to apply to the isochorlc heating situation. Early thinking about isochoric heating led to the ldea that relaxation from the compressed state could rapidly move a liquid into the negative pressure region under the liquid-vapor two phase dome on a P-V diagrem. If the consequent vaporization is rapid enough, the expanding vapor bubbles could drive the fragmentation process. In this work, the lithlum equation of state was used to demionstrate that veporization caused by the neutron heating uses only a minor fraction of the added heat and that fragnentation would occur due to hydrodynamic motion, even in a pure liquid that had no vaporization. In addition, it was found that a $77 \%$ expansion of the lithium was requirad before the rapid veporization process of spinodal dacomposition could begin, and that vapor bubble growth is slower then the hydrodynamic expension. Therefore, it is concluded that rapid vaporization is not the driving machanism for liquid fragnentation in ICF reactors. 
An energy mininization procedure was used to determine the fragment size that would produce the least amount of non-fragnent-center-of thass energy, 1.e., the sum of the surface and dilational kinetic energies. This modal (tive Grady-Glenn model) predicted that fragment radius, a, depended on the original system radius, $R$, and the mount of isochoric heating, (In energy por unit mass units), as will as on four liquid properties: density $\left(p_{m}\right)$, Grönelsen parameter $(\Gamma)$, surface tension $(\gamma)$, and sound spaed (c). Use of the model predicted considerable fragnentation in the high energy density HYLIFE reactor and minimum fragnentation in low energy density reactors like Pulse*Star, EARLE, and HIBAL.

A two-dimensional molecular dynemics model was developed to test the Grady-Glenn model at a misroscopic scale for the hypothetical Lennard-Jones fluid. The two Lennari-jones constants, of and $\varepsilon$ were chosen to represent lithlum, and tine requized liquill propertles were generated from published equation of state jata and from additional molecular dynamics calculations. A two dimansional model was used because it most closely models a uniformly haated cyllindrical jet, because it permits easy graphlcal inspection of the atomic configurations, and because it allows consideration of larger radius systems (the molecular dynarics calculations were in a size regime where the surface tension properıy was a function of the system radius). Molecular dynamics calculations were run using five leveis of heating and four system sizes. The numerical experiments produced reasonable agreenent with the Grady-Glenn model and poor agreement with three other hydrodynamic models. The power law dependence of fragment size (in atons) on systen size and heating level was correct 
$\left(N_{c} \sim N^{2 / 3} p^{-20 / 3}\right.$ ) where $D$ is the number of dimensions, and the larger fragient sizes were within an order of magnitude of the predictions. The potentlal error in the system size dependence (from the priedicted $\mathrm{N}^{2 / 3}$ down to $\mathrm{N}^{1 / 2}$ ) would produce an error of a fector of $\sim 25$ at the HYIFE scale. Additional analysis of two Italien three dimensional molecular dynemics experiments also agreed with the model predictions.

For ICF reactor desion, the result of this study is that the Grady-Glem fragment radius predictions should be reduced by a factor of $10^{1 / 0}$. Thus, the design equation becores

$$
a=10^{1 / D}\left[\frac{D+2}{P_{m}} Y\left(\frac{R C}{\Gamma e}\right)^{2}\right]^{1 / 3}
$$

where a indicates the radius of the larger fragments (smaller fragnents are also expected). However, the excess kinetlc energy generated in the simulations (ove to fluctuations and vapor pressure formation) is probebly correlated with the smaller than predicted freyment size. Consequently, at macroscopic sizes, the factor of $10^{1 / 0}$ could disappear. In either case, a prediction within a factor of -2 would certainly be acceptable. For the HYLIFE Inner jet conditions, Eq. 8.1 predicts a fragment radius between 1 and $2 \mathrm{~mm}$, leading to a two order of magnitude increase in the liquid surface area. $=$

There wa a fair emount of uncertainty in the molecular dynanics rewulls since the freguntation is driven by atomic motion, and, in such mill systems, fluctuations in statisticel quentities enong the verieus atoms ind in tiw are much more sionificent then for 
macroscopic systems. These fluctations are expected to prodice smaller fragments then predicted by the equilibrium model. It is expected that, if many nore molecular dynemic runs ware mada using slightly different initial conditions, the ensemble results would also match the model somemat more closely.

Although the molecular dynemics results demonstrated that the Grady-Glem power law formulation is correct at the microscopic scale, the huge degree of extrapolation to reactor slze makes it prudent to conduct macroscopic (laboratory rather than numerical) experiments before reactor construction is authorlzed. Saveral potential experiments using non-fusion sources have been proposed ${ }^{2}$. Thase experiments include adding the heat with pulsed laser or microwave illumination, capacitor discharge through the liquid, and driving a diverying shock through the liquid with either explosives or mechanical impact. Each method appears to be capable of adding enough heat or momentum to a small system in a short enough time, but each method also has possible complications.

Laser Illumination can result in non-uniform energy deposition at a very small scale, 1.e., at the peaks in the diffraction pattern. In the Soviet Union, liquids Illuninated with lasers have exploded, and the data indicate that the bean absorption was concentrated in very small spots wich then flash vaporized ${ }^{67}$. Capacitor discharges through liquid jets can have non-uniform heating due to the tendency of pulsed currents to travel along the outer surface of the conductor (skin effect). Further, the pulsed current generates a magnatic field that interacts with the current to produce an inmerd directed Lorentz force (pinch effect). Shock experiments include a constant outward 
wlocity and en initial pressure profile peoked at the center, as compared to isochoric heating which has zero initial velocity and either a constent or a one-surface peaked initial pressure profile. It my be possible to simulate some of the complicating effects in molecular dymics simulations. In eny case, at least two types of experitments should be performed to facilitate separation of spurious effects rrom the desired results.

The molecular dynemics simulations can also be extended. One stralghtforwerd extension would be to repeat the calculations from different equilibrated configurations. Another extension would be to consider non-uniform isochoric hating. Finally, it might be possible to begin with en equilibrated surrounding vapor contained within a larger container that reflects only small clusters. 


\section{Poforences}

1. E. W. Sucov, D. A. SInk, L. Grwen and H. R. Howlend, "EPRI Worksiop on Evaluation of Engineering Opportunities in Inartial Confinement Fusion in Existing and Plemned Facilitles, "Electric' Power Research Institute Report AP-2317 (1902).

2. N. J. Hoffmen, J. A. Blink, B. G. Jones, J. F. Stubbins and D. Tichenor, "Oissassembly of Isochorically thated Liquids-final Report to EPRI", Eneroy Technology Engineering Center Report EPRI/OIHL (1983).

3. M. J. Monsler, J. Hovingh, D. L. Cook, T. G. Frank and G. A. Moses, "An Overview of Inertial Fusion Reactor Design," Nucl. Technol./Fusion, 1, p. 302, Sec. IX, (1982). See also Lawrence Livermore National Laboratory Laser Program Annual Repcrts for 1978, 1979, 1980, and 1981 (UCRL-50021-year, where year is $78,179,180$, or 81 ).

4. L. A. Glenn, "ICF Reactor Chamber Coolinis Following the Fusion Microexplosion," Lawrence Livermore National Laboratory Internal Meno (Alg. 10, 1978).

5. L. A. Glenn, "Dynemic Loading of the Structural Wall in a Lithium-Fall Fusion Reactor," Nuc. End, and Dession, 59, p. 1 (1979).

6. L. A. Glenn, "Divergent Impulsive Crossflow Over Packed Columar Arrays," Muc. Eno, end Dastion, \%, p. 429 (1900).

7. L. A. Glern, "On the Motion Following Isochoric Heating of Concentric Liquid Annuli," Lewrence Livermore Matlonal Leboratory Report UCRL-a4003 (1900). 
8. L. A. Glem, "Transport Processes in an Inertlal Confinement Fusion Rasctor," Muc. Enq. and Desion, 64, 3, p. 375 (1981).

9. B. Badper, et al., "HIBAL -A Conceptual Heavy Ion Beam Driven Eusion Rector Study," University of Wisconsin Report UwFOM-450 (1901).

10. L. C. Pong, D. K. Sze, R. R. Paterson and G. A. Moses, "Progress on Modaling of $\mathrm{Pb}_{83}{ }^{\mathrm{l} .1_{1}}$, Recondensation on INPORT Units," Fusion Powrer Associates Report FPA-82-5 (1982).

11. J. H. Pendergrass, T, G. Frank and L. O, Bohachevsky, "A Modifled wetted-wall Inert1al Fusion Reactor Concept," Proceedings of the 4th Tool. Mto. Iechnolooy of Controlled Nuclear Fusion, King of Prussia, Penn., p. 1131 (1981).

12. W. O. Allen, et al, "Phase I: Englneering Test Reactor Goal Specification Final Report," Electric Power Research Institute Report EPRI-1527 (1982).

13. J. A. Blink and N. J. Hoffmen, "Tritium Control and Activation in the Pulse"Star Reactor," Proceedings of the IEEE 10th Symposium on Euston Enoinaring, Philadelphla, Penn. (1983).

14. E. Giffen and A. Muraszew, The Atomization of Liquid Fuels, Chapran and Hall, Ltd., London, England (1953).

15. R. D. Raltz and F. V. Bracco, WUltra-High-Speed Fllming of Atomizing Jats," Phys, Fluids, 22, 6, p. 1054 (1979).

16. R. D. Raitz and F. Y. Bracco, Mpochenlsm of Atomization of a Liquid Jat," Phys. Flutids, 25, 10, p. 1730 (1982).

17. D. A. Young, "A Soft-Sphere Model for Liquid Metals," Lawrence Liverwore National Leboratory Report LCRL-52352 (1977). 
18. L. A. Glern, "AFION Revisited: An Improved Algorlthm for Munerical," Solution of Initial Value Problems in Continumen Mechanics. Part 1: The One-Dimensional Equations," Lawrence Livernore National Laboratory Roport UCRL-52512 (1978).

19. W. G. Hoover, G. Stell, E. Goldmark and G. D. Dageni, "Generallzed van dor Walls Equation of State," J. Chem. Phyclics, 63. 12, p. 5434 (1975).

20. G. Pelerson and D. Chato, "Mathsy Manual," Lawrence Livermore National Laboratory Report UCIO-18875 (1982).

21. J.A. Blink, "Lithium Equation of State," Lawrence Livermore National Laboratory Report UCID-19890 (1983).

22. J. A. Barker, D. Henderson and F.F. Abrahem, "Phase Dlagrem of the Two-Dimensional Lennard-Jones System; Evidence for First Order Transitions", Physica, 106A, p. 226 (1981).

23. F. F. Abrahem, "The Phases of Two-Dimensional Matter, Their Transitions, and Solid-State Stability: A Perspective via Computer Simulation of Simple Atornic Systems", Physics Reports, 80, 5, p. 339 (1981). This reference also contains a large bibliography of two-dimensional simulation studies.

24. D. Henderson, mante Carlo and Perturbation Theory Studios of the Equation of State of the Two-Dimensional Lennard-Jones Fluid," Molecular Phys., 34, 2, p. 301 (1977).

25. J. M. Phillips, L. W. Bruch and R. D. Murphy, "The Two-Dimensional Lennard-Jones System: Sublimation, Vaporization, and Melting," J. Chem, Phys., 75, 10, p. 5097 (1981). 
26. F. Tsien and J. P. Valleau, "A Monte Carlo Study of the Two-Dimensional Lemard-Jones System," Molecular Phys., 27, 1. p. 177 (1974).

27. P. Fehder, Molecular Dynemics Studies of the Microscopic Properties of Dense Fluids," ‥ Chem. Phys., 50, 6, p. 2617 (1\%9).

28. S. Toxyaerd, "The Mobile-Layer Model for Absorption," Molecular Physe, 29, 2, p. 373 (1975).

29. S. M. Thompson, K. E. Gubbins, J.P.R.B. Walton, R.A.R. Chantry and J. S. Rowlinson, "A Molecular Dynemics Study of LIquid Drops", 1. Chem. Phys., 81, 1, p. 530 (1984).

30. J. H. Cahn, "Spinodal Decomposition," Transactions of the Metallurgical Society of AIME, 242, p. 166 (1968).

31. R. C. Reid, "Superheated Liquids," American Scientist, 64, P. 146 (1976).

32. J. H. Cahn, "Phase Separation by Spinodal Decomposition in Isotropic Systems," I. Chem. Phys., 42, 1, P. 93 (1965).

33. H. R. Mruzik, F. F. Abrahem and G. M. Pound, "Phase Separation In Fluid Systems by Spinodal Decomposition II: A Molecular Dynemics Computer Simulation," J. Chend. Phys., 69, 8, p. 3462 (1978).

34. E. Gerum, J. Straub and U. Grigull, "Examination of the Motastable State of Surface Tension, Coexistence," that Transfer in Bolling, E. Hahne and U. Grigull editors, Academic Press, New York, Ch. 19 (1979).

35. J. Frenkel, Kinetic Theory of Liquids, Dover Publications Inc., Now York, 2nd odition (1946). 
36. W. G. Hoover, A. C. Hindnarsh and B. L. Holien, Number Dependence of Sinall-Crystal Thermodynanic Propertles. I," 1. Chem. Phys., 57, 5, p. 1980 (1972).

37. H. M. Kottowsk1, "The Mechanism of Nucleation, Suporheating, and Reducing Effects on the Activation Energy of Mucleation," Proaress in thent and Mase Trensfer, 2, (thet Trensfer in Liouid Metals), Perganon Press, P. 299 (1977).

38. E. N. Brodskaya and A. I. Rusanov, "Investigation of $\sin l 1$ Systems by Molecular Dynemics Mathod. 2. Pressure Tensor and Surface Tension of Small Drops," Collold Journal of the USSR, 39, 4, p. 646 (1977). See also S. I. Rusanov and E. N. Brodskaya, "The Molecular Dynamics Simulation of a Snall Drop," I. of colloid and Interface Science, 62, 3, P. 542 (1977).

39. R. E. Henry and K. Niguzakl, "Effects of Systen Pressure on the Bubble Gruwth from Highly Superheated Water Droplets", Iopics in Two-Phase Heat Transfer and Flow, ASME Winter Annual Meeting, San Francisco, Cal., p. 1 (1978).

40. L. J. Briggs, "Limiting Negative Pressure of Water," J. Appl. Phys., 2l, p. 721 (1950).

41. L. J. Briggs, Maximm Superheating of Water as a Measure of Negative Pressure," ]. Aopl. Phys., 26, p. 1001 (1955).

42. S. J. Henderson and R. J. Speedy, "A Berthelot-Eourdon Tube Melliod of. Studying Water Under Tension," J. Phys. E, 13, p. 778 (1980). 
43. F. F. Abrahan, D. E. Schreiber, M. R. Mruzlk and G. M. Pound, "Phase Separation In fluid Systems by Spinodal Decomposition: A Molecular-Dynamics Simulation," Phys. Rev. Letters, 36, 5, p. 261 (1976).

4h. J. Hovingh, M. Thomson, and J, Blink, "Fluid Mechanlcs Considerations for Liquid Wall Inertially Confined Fusion Reactors," Proceedings of the IEEE ath Symo. on Enqineering Problems of Fusion Research, p. 2038 (1979).

45. D. E. Grady, "Local Inertial Effects in Dynamic Fragmentation," 1. Aopl. Phys., 53, 1, p. 322 (1982).

46. L. A. Glenn, "On the Fragmentation of Condensed Material by Isochoric Heating and Release," Lawrence Livermore National Laboratory Report UCID-19737 (1983).

47. F. A. McClintock and A. S. Argon, editors, Mechanical Behavior of Moterials, Addison-Wesley Publ. Co., Reading, Mass., p. 489 (1966).

48. F. F. Abrahem, W. E. Rudge, D. J. Auerbach and S. W. Koch, Molecular-Dynamics Simulations of the Incommensurate Phase of Krypton on Graphite Using More Than 100,000 Atoms," Phys. Rev. Let., 52, 6, p. 445 (1984).

49. W. G. Hoover, "Computer Simulation of Many-Body Dynamics", Physics Today, 37, 1, p. 44 (1984).

50. W. G. Hoover, "Nonequilibrium Molecular Dynamics," Ann. Rev. Phys, Chen., 34, P. 103 (1983).

51. F. Gibb and $w$. Smlth, "CCPs Literature Survey," Information Qunterly for MD \& MC Simulations, 8, Daresbury Laboratory, Daresbury, Warrington, England, p. 49 (1983). 
52. E. N. Brodskaya and A. I. Rusanov, "Investigation of $\sin L 1$ Systems by Molecular Dynanles Method. 1. Energy and Molecular Structure of Snall Drops," Collotd Journal of the USSR, 39, 4, p. 561 (1977).

53. B. J. Alder and T. E. Walnwright, "Studies In Molecular Dynanics. I. General Method," J. Chem. Phys., 31, 2, p. 459 (1939).

54. L. Verlet, "Computer 'Experiments' on Classical Fluids. 1. Thermodynamical Properties of Lennard-Jones Molecules, " Phys. Rev, 159, 1, P. 98 (1967).

55. S. M. Thompson, "Use of Neighbor Lists In Molecular Dynamics" Information quarterly for MD \&C Simulations, 8, p. 20, Daresbury Laboratory, Daresbury, WarrIngton, England (1983).

56. J. C. Slater, Introduction to Chemical Physics, McGraw-HIll Book Co., New York, p. 217 (1939)

57. C. A. Croxton and R. P. Ferrier, "Statistical Mechanical Calculation of Surface Propertles of Simple Liquids: IV. Molecular Dynemics," J. Phys. C, 4, p. 2447 (1971).

58. J. S. Rowlinson and B. Widom, Molecular Theory of Caoillarity, Clarendon Press, Oxford, p. 177 (1982).

59. A. H. Falls, L. E. Scriven and H. T. Davis, "Structure and Stress in Spherical Microstructures," ㄱ. Chem. Phys., 75, 8, p. 3986 (1981).

60. J. H. Irving and J. G. KLrkwood, "The Statlstical Mechanical Theory of Transport Processes. IV. The Equations of Hydrodynamics," J. Chen. Phys., 18, 6, p. 817 (1950). 
61. A. Harasima, "Molecular Theory of Surface Tension," Ady. Chem. Phys., 1, p. 203 (1958).

62. S. Ono and S. Kondo, Molecular Theory of Surface Tension in Liquids," Encyclopedia of Physlcs (Handbuch der Physik), edited by S. Flogge, Springer-Verlag, 10, Pf, 157-161 (1960).

63. A. Vicentinl, G. Jacuccl, and V. R. Pandharipande, University of Illinols, in preparation for Phys. Rev, C.

64. S. W. Hoch, R. C. Desal and F. F. Abraham, "Dynamics of Phase Separation In Two-Dimensional Fluids: Splnodal Dacomposition," Phys. Rev. A, 27, 4, p. 2152 (1983).

65. F. F. Abraham, "On the Thermodynamics, Structure and Phase Stabllity of the Nonuniform Fluid State," Physics Reports, 53, 2, p. 93 (1979).

66. F. H. Ree, "Analytic Representation of Thermodynamic Data for the Lemard Jones Fluld," J. Chem. Phys., 73, 10, p. 5401 (1980).

67. V. S. Loskutov and G. M. Strelkov, "Explosive Evaporation of Wakly Absorbing Drops Under the Action of Laser Pulses", Publication of the Institute of Radice Engineering and Electronics, USSR Academy of Sclences, Moscow, p. 1 (1980). Lawrence Livermore National Laboratory Translation UCRL TRANS-11809 (1982).

6B. H. Schulz, B. KEmpler, H. W. Barz, 6. Röpke and J. Bondorf, "On the Time-Development of the LIquid-Yapor Phase Transition in an Expanding Muclear System," Phys, Let.ters, 1478, 1-3, p. 17 (1 Nor 19a4). 
Nomenclature
Helmholtz free energy
$A^{\mathrm{e}}$
excess Holmholtz free energy
A
hexagonal-close-packed base-plane triangle area at melt
$A_{1}-A_{5}$
curistants used In Ree's pressure equation
A.
Helmholtz free enargy per atom In an Infinite system
$A_{\infty}^{*}$
free energy per atom in an Infinite system plus the excess
entropy due to surfaces
a
fragment radtus
$\mathbf{a}_{\mathbf{s}}$
radius at which the surface tension applies
B
isentropic bulk modulus
$\mathbf{B}_{\mathrm{m}}$
isentropic bulk modulus at tise melting point
B
isothermal bulk modulus
$B_{1}-B_{4}, B_{10}$
constants used In Ree's pressure equation
b
curvature of the parabolic potential
C
concentration (for spinodal decomposition)
$\mathbf{C}_{\mathrm{A}}$
concentration of phase A (for spinodal decomposition)
$C_{n}$
face centered cublic Madelung constant
$c_{1}-c_{5}$
constants used in Ree's pressure equation
C
sound speed
c*
density-normalized sound speed
c.
liquid specific heat at constant pressure
$\mathbf{c}_{\mathbf{p}}$
specific heat at constant. pressure
$c_{y}$
specific heat at constant volume
number of dimensions

D 

$D_{1}$ constant used to distinguish 2-D and 3-D results. The subscript is incremented as the constant is redefined in the derivation.
$D_{n} \quad$ diffusion coefficient
d Interatomlc spacing for a perfect crystal (at a given density)
d Initial interatomic spacing for a crystal
F. Internal energy, Internal energy per atom
$E_{\text {coh }}$ cohesive energy, the energy required to vaporize room temperature ifthlum to the critical polnt
e
$e^{*}$
$\vec{F}, \mathbf{F}$
$\vec{F}_{1 j}$
$F_{k}$
specific energy (energy per unit mass) added suddenly
2.71828 (base for natural logarithms)
interatomic force
force on atom 1 due to atom 3
normal component of the intriatomic force of the $k$ 'th atom pair

$F_{p}$

$\mathbf{f}_{1}$

G

$\mathbf{g}_{\mathbf{j}}$

$H_{\text {m }}$

$\mathbf{H}_{\mathbf{v}}$

n

I

J

$\mathbf{J}_{\mathbf{1}}$ function used to convert the sound speed from one density to arrother

constants used in Barker's pressure equation

Gibus free energy per unit mass

constants used in Barker's pressure equation

enthalpy at melt

heat of veporization

Plenck's constant

noment of Inartla

diffusional flux (for spinodal decomposition)

diffusional flux of phase 1 (for spinodal decomposition) 


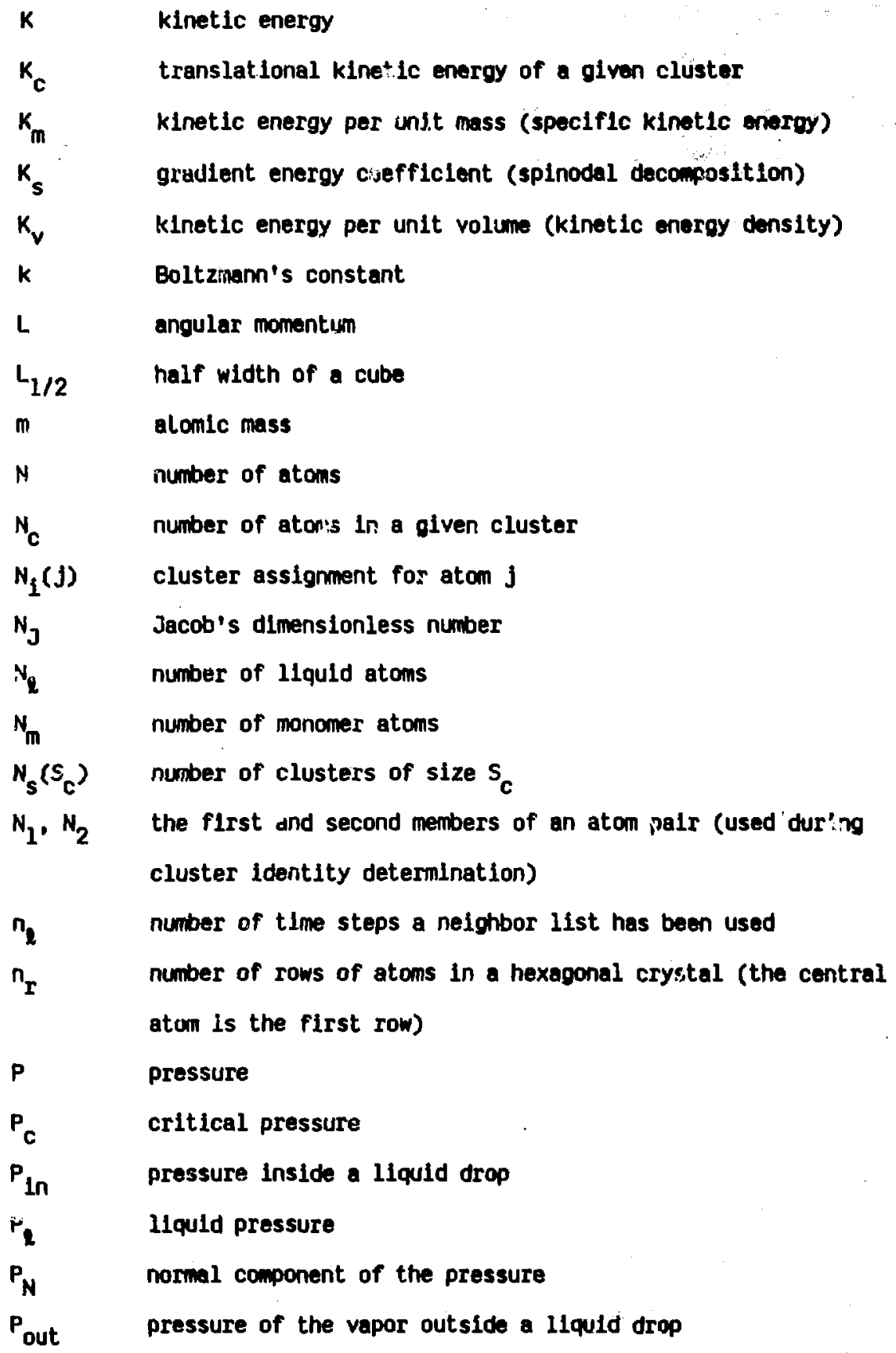




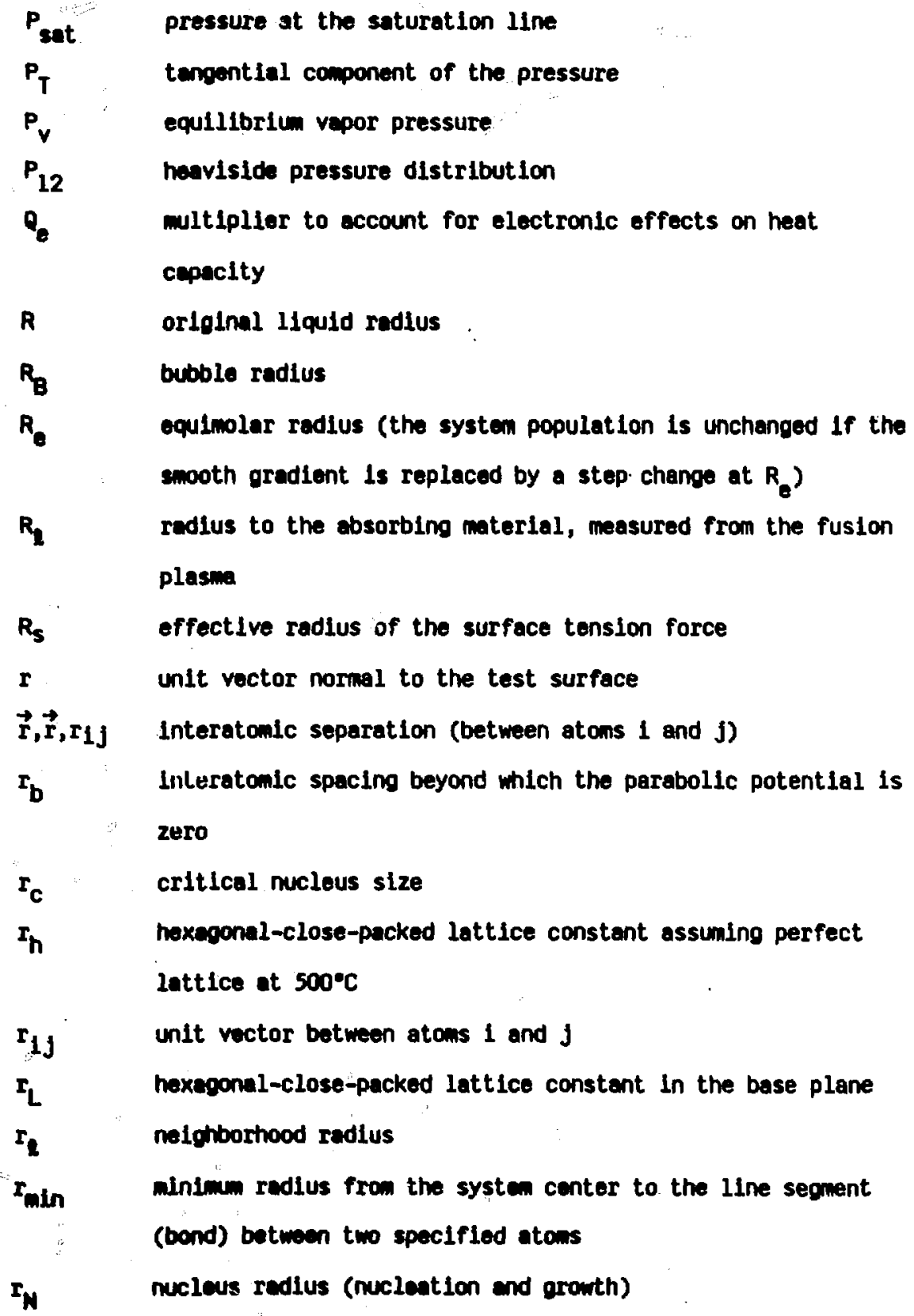




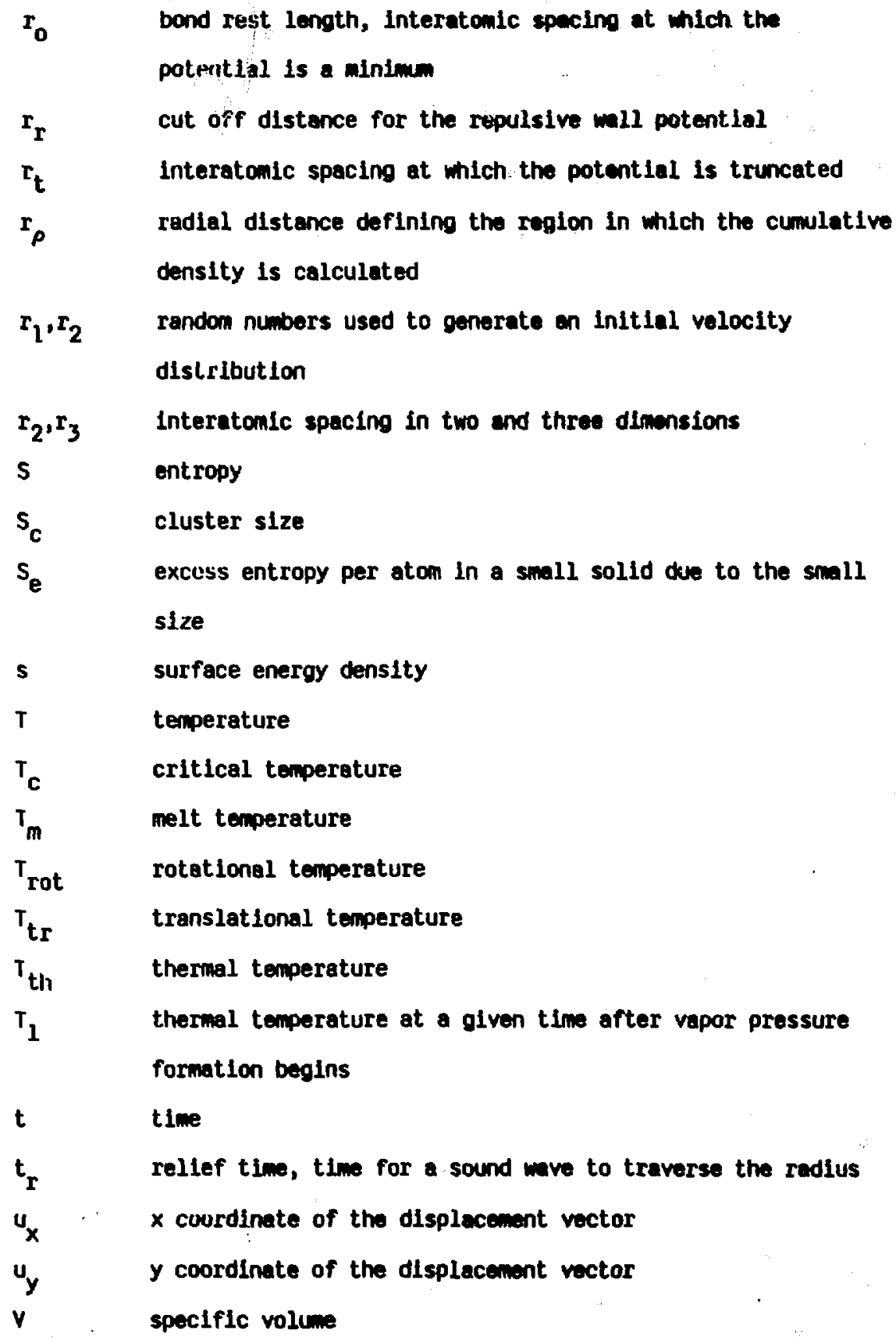




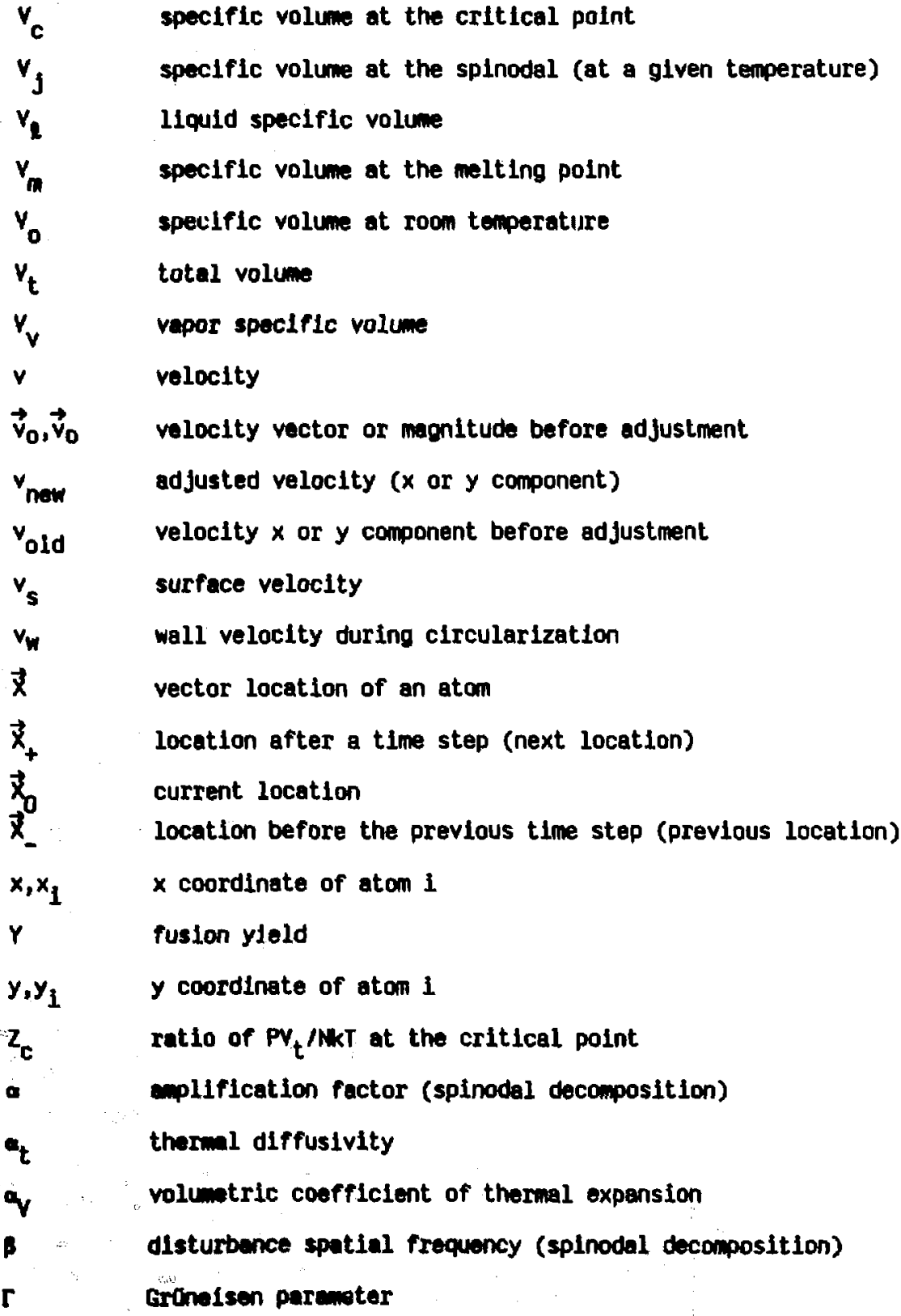



r cumulative Groneisen paremeter from saturation to the heated state
$\Gamma_{1} \quad$ Groneisen parameter for mode 1 in a solid
$r_{0} \quad$ Gronelsen parameter at room temperature
$Y, Y_{\text {. }} \quad$ surface tension in a large system (independent of systen size)
$\mathbf{Y}_{\mathbf{S}}$
$r_{2}, r_{3}$
$\Delta P$
$\Delta T_{\mathbf{l}}$

$\Delta t$

c

$\varepsilon_{x x}$

Exy

cy

$n$

r

$\lambda$

$\lambda_{\mathbf{I}}$

Ad

$\left|\lambda_{\min }\right|$ value of $\left|\left(2 \vec{r}-\vec{r}_{1}-\vec{r}_{j}\right) / \vec{r}_{1 j}\right|$, when the radius to the extended line between two specified atoms is minimized 
dimansionless constant used in the disassembly velocity profile

$\boldsymbol{u}_{1}$

chemical potential of phase 1 , (spinodal decomposition)

$\boldsymbol{\lambda}_{\mathbf{t}}$

values of $\lambda$ at the two Intersections between a circular arc and the extended line between two specified atoms

$\boldsymbol{v}$ fragment size correction factor that accounts for varlable velocity divergence within the original drop

vibration frequency 1 in a sol.ld

exponent for the lithlum equation of state repulsive potent. lal

$\pi$

\subsection{9}

p

atom density

$P_{C}(r)$ cumulative atom density from the center to radius $r$

$P_{m}$

Py

$P_{\text {nn }}$

p*

-

$\mathbf{T}$

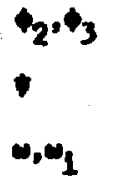

mass density

Young's dimensionless atomic density, $\mathrm{No}^{3} / \mathrm{r} 2 \mathrm{~V}$

mass density of the liquid

normalized atom density

interatomic separation at which the Lennard-Jones potentlal

1s zero

time for an expansion wave to travel from the surface to the center

Interatomic potential energy

volume fraction of absorbing material in a reactor bsorbing region

potential energy in two and three dimensions

repulsive potential ensrgy at $r=\lambda$

vibration engular frequencies in a solid 


\section{Appendix A}

This appendix is a draft of a journal article summeizing the study; as such, it may be read as en executive summary. It contains an independent set of figures for corvenience, and it uses its own nomenclature (dofinad in its text). However, it cltes the refarences from the main body of the document.

\section{A.1. Abstrect}

The rapid fragmentation of two-dinensional high-pressure disks of Lennard-Jones fluid was studied using molecular dynamics. The free expansion of 169,721, 2611, and 14491 particle systems was studied for several sound traversal times. The breakup into fragments (or clusters) can be roughly described by Grady's model, which balances the surface energy with the comoving (dilatlonal) kinetic energy of the fragments. The model predicts that the number of fragments varies as the cube root of the system population and the $20 / 3$ power of the initial pressure were $D(2$ or 3 ) is the dimensionality of the system. The molecular dynamics results confirm these predictions within about \pm 0.1 and thereby rule out three other fragmentation models. The relatively high nonomer temperatures and relatively uniform fragment temperatures found here correspond to temperatures found in recent three-dimensional Lennard-Jones simulations by VIcentIni, Jacucci and Pandharipande.

\section{A.2 Introduction}

The expension of high-pressure hot fluid into a low-pressure 
volume many times the original volume is a strongly nonequilibrium process. Wave propagation, fluctuations, negative pressures, surface tension, vaporization, viscosity, and heat conduction all play roles as a single fluid mass is converted to a time-dependent collection of - diverging liquid and solid fragnents or clusters. A complete theoretical or computational study of such a process lies well beyond our capacity. Thus, fragmentation is primarily "understood" on the basis of experimental correlations and relatively crude "theorles" (models). The expansion of an 1sochorically heated fluid is a challenging problem involving non-equillbrlum statlstical mechanics, dynanics, and fluid nechanics. Three diverse fields provide sample applications whlch could benefit from the ability to predict fluld fragmentation. Lithium jets in Inertial Confinement Fusion reactors are heated by neutrons much more rapidly than the tens of mlcroseconds requiced for pressure rellef. Pressurized fluids leaving nozzles frabment ${ }^{16}$. Colliding heavy ion beams produce compressed "nuclear matter" wich exhlbits fragmentation and a vapor-liquid like phase transition 68 .

wile moen fragnent size is a crude description of the fragmentation process, it is easily measured and calculated. Four hydrodynamic models have been proposed to predict the mean fragnent slze. Two of these models balance fragment surface forces against pressure, either ( 1 ) the increased static pressure $P$, or (2) the dynemic expension pressure (mpv $2 / 2)$ where $m$ is the atomic mass, $p$ is the atom density, and $v$ is the Jump-off velocity, $P / m p c$, with c Indlcating the sound speed. Two other models minimize the sum of the frament surface and dilational onergies, consldering (3) the 
total fragment dilational energy, or (4) the dilationil energy near. the fragment surfaces. These four models have markedly different dependences on the original system size and pressure.

Vaporization has also been proposed as an expansion and fragmentation mechenism. Two phase chenge machenisms are "spinodal decomposition" 30 and "nucleation and growth". Naither mechanism suggests any depandence of mean fregment slze on original system size.

Molecular dynemics provides a test of the size dependence predictions of the various theoretical models. Hare, we apply molecuiar dynanics to a two-dimensional system because it can simulate relatively large radius systens, because it is easily diagnosed graphically, and because it best simulates the quasi-two-dimensional expansion of cylindrical jets in inertial fusion reactors.

\section{A.3. Fragmentation Models}

\section{A.3.A. Hydrodynemic Madels}

The simplest model requires the surface tension ( $2 \gamma$ or $2 \pi r Y$ in 2- or 3-D) to balance the inltial pressure, $2 \pi \mathrm{P}$ or $\pi r^{2} \mathrm{P}$. Thus, the "fragment" or "cluster" size, $N_{c}$ (In atoms) is

$$
N_{c}=r\left(\frac{D+1}{D}\right)^{D-2} \rho\left[\frac{(D-1) X_{1}}{P}\right]^{D}
$$

where the dimensionality, 0,15 either 2 or 3 . A nore realistic model accounts for the relief of the static pressure, producing a dynamic expansion pressure and replacing $P$ in Eq. A.I by mov $2 / 2$, where $v$ is the Jump-off velocity,

$$
N_{c}=\left(\frac{D+1}{D}\right)^{D-2} \rho^{D+1}[2(D-1) Y]^{D}\left(\frac{c}{p}\right)^{2 D} .
$$


An energy minimization model (proposed by Grady ${ }^{45}$ and extended by Glem ${ }^{46}$ and (his study) minimizes the sum of the surface energy density plus the fragment dilational energy density. The surface energy density is

(OY/r) where $I$ is the fragment radius. For a homogeneous expansion, the dilational energy density is $(r \dot{\rho})^{2} /[2 D(D+2) \rho]$ where $-\dot{\rho} / \rho=D v / R$. Thus

$$
N_{C}=\pi^{1 / 3}\left(\frac{D+1}{D}\right)^{(D-2) / 3} p^{(D+1) / 3}[(D+2) Y]^{D / 3}\left(\frac{g}{p}\right)^{2 D / 3} N^{2 / 3} .
$$

The inhomogeneity of the expansion can be partly considered by including the radial dependence of the velocity divergence. Then, the fragment slze predicted by Eq. A.3 is reduced by one to two orders of magnilude for the innermost fragments, but most of the system mass is included in fragments within a factor of two of the size predicted by the uncorrected Eq. A.3.

If only the dilational energy in an outer shell of thickness is used in the energy minimization model, $N_{c}$ is proportional to $N_{\text {. }}$

$$
N_{c}=\left(\frac{2 e r}{\delta}\right)^{D / 2}\left(\frac{c}{p}\right)^{D} N
$$

If Eq. A.3 proves to be a more nearly correct model than Eq. A.4, frabmentation must be driven by collective effects, Including energy in life fragment core as well as the energy at the fragnent surface.

In sumary, the hydrodynemlc models, (1), (2), (3) and (4), predict $N_{c} \sim N^{0} P^{-2}, N^{0} P^{-4}, N^{2 / 3} P^{-4 / 3}$, and $N^{-2}$, respectively

In lwu dimensions,. In three dimenslons, the four models predict $\mathrm{N}_{\mathrm{c}}$ $\sim \mathrm{N}^{\mathrm{O}} \mathrm{P}^{-3}, \mathrm{~N}^{\mathrm{O}} \mathrm{P}^{-6}, \mathrm{~N}^{2 / S_{\mathrm{P}}-2}$, and $\mathrm{NP}^{-3}$. 


\section{A.3.8. Phase Conere as a Fragnentation Machenisu}

It is easy to see that phase change is too slow and too meak a mechanism to drive fragmentation. For example, Flg. A.l shows the response to $800 \mathrm{~kJ} / \mathrm{kg}$ of isochoric neutron heating in a 0.2 m diemeter lithium jet in the HYIFE ${ }^{3}$ ICF reactor, using Young's soft-sphere equalion of state ${ }^{17}$. The sudden heating causes the liquid temperature $\left(500^{\circ} \mathrm{C}\right)$ and pressure (essentlally zero) to quickly rise to $735^{\circ} \mathrm{C}$ and $380 \mathrm{MPa}$ with no change in density $\left(485 \mathrm{~kg} / \mathrm{m}^{3}\right)$. As the relluf wave moves into the jet, a naarly isentroplc expansion occurs. During expansion to the saturation line (and $P_{\text {sat }} \sim 0$ ), the liquid temperature falls to $695^{\circ} \mathrm{C}$, and the internal energy falls by $\sim 16$ $\mathrm{kJ} / \mathrm{kg}$. This energy corresponds to a jump-off velocity of abnut 175 $\mathrm{m} / \mathrm{s}$ as the internal energy is converted to kinetic energy by the tensile wave. When the expansion continues beyond zero pressure into the two-phase liquid-vapor region, phase separation or fragmentation must eventually occur, returning the fluid to the saturated liquid and vapor lines. If the isentroplc expansion could continue all the way to the isentropic spinodal (where $[\partial P / \partial Y]_{S}$ vanishes), only 3.4 $\mathrm{J} / \mathrm{kg}-\mathrm{llq}$ quid would be needed for equilibrium vaporization. This is about $\mathbf{4 4 0 0}$ times less energy than the jump-off kinetic energy produced by the hydrodynanic expansion. Thus, vaporization ut.1.11zes insufficient energy to drive the hydrodynamic expansion and fragmentation.

It is unlikely that either spinodal line would even be approached in a real liquid lithium ICF reactor. At the spinode, the liquid tension is $\sim 1560 \mathrm{MPa}$, much larger than the tensile strength of solid lithium. In an ICF reactor, the entrained gas, target debris, 
Fig. A.1. P-V diagrem of the heating and expansion of a lithlum jet. Due to the restricted specific volume range of the diagram, the coexistence curve appears to be flat at $\rightarrow$ pressure. The critical pressure of $114 \mathrm{MPa}$ is reached at $0.011 \mathrm{~m}^{3} / \mathrm{kg}$, well to the right of the portion of the diagram stiown. The isothermal and isentropic spinodal lines cross the lower right corner of the graph.

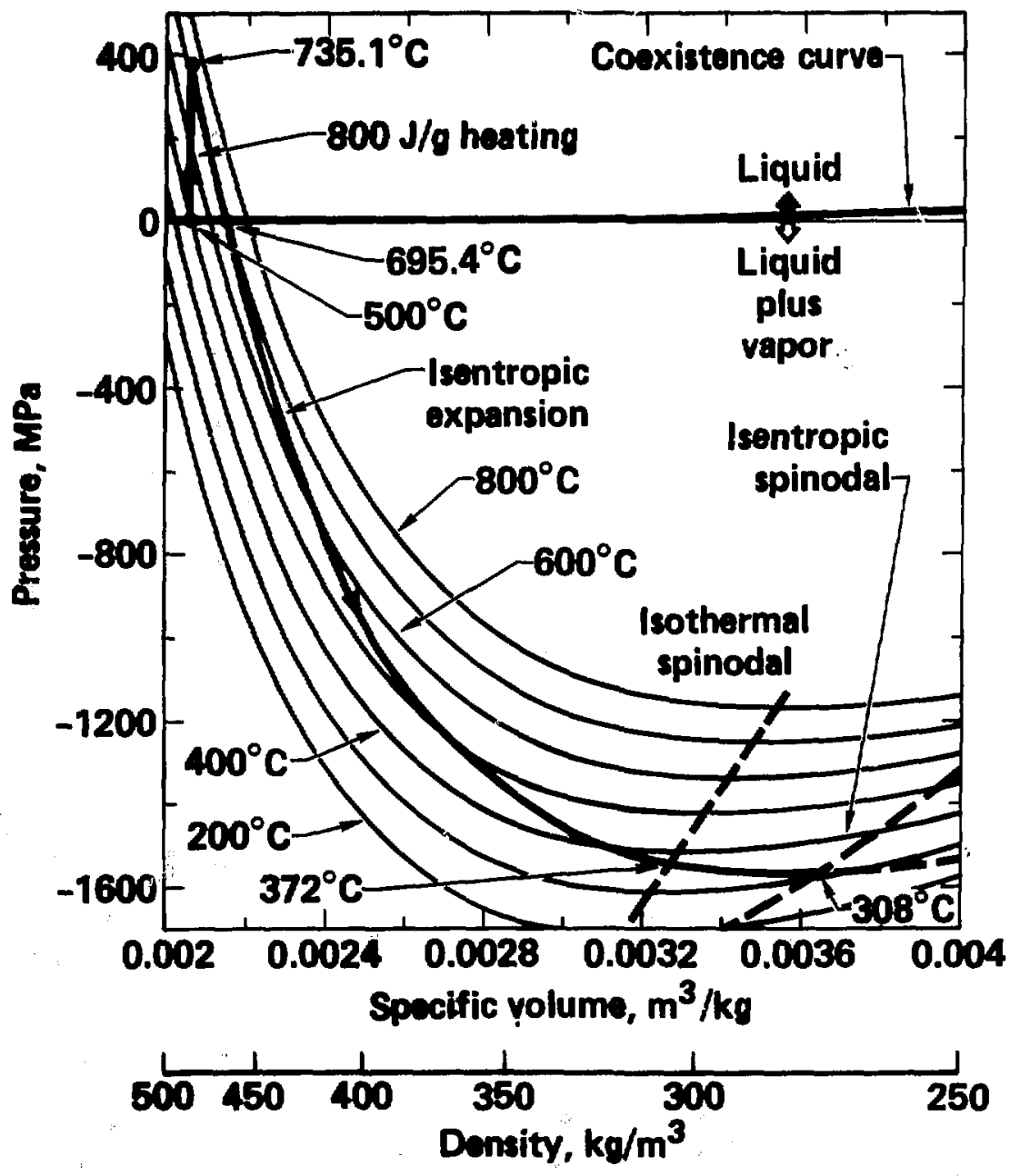


and corrosion products provide an abundance of potential mucleation sites. Vapor bubble growth (fron nucleation and growth) is also Ineffective from a kinetic standpoint since the growth times (milliseconds) are much longer than the liquid's hydrodynamic expansion time. Hence, it is concluded that hydrodynamic fracture is the fragmentation mechanism and that phase separation occurs later to fill the consequent voids.

\section{A.4. Molncular Dyneulics Model of Fromentation}

The well-characterized Lenriard-Jones 6-12 potential was used

$$
\underset{c}{\varphi(r)}=4\left[\left(\frac{\sigma}{r}\right)^{12}-\left(\frac{\sigma}{r}\right)^{6}\right] .
$$

The potential was truncated at $r_{t}=2.5 \sigma$ to save computer time. The truncation has negliglble effect on the evolution of the system, but it does allow early identification of fragment sizes. In addiliun, Thompson's nelghbor listing procediture ${ }^{55}$ was used to reduce computer time, at some memory expense. The largest run (14491 atoms) required a menory of 212,000 words and 6.3 Cray CPU sec for each D.003 ps time step. In implementing Thompson's scheme, a 11st was conslructed (for each atom) that Included all atoms within some range $r_{l}>2.5 \sigma$. After the list was complied, the cumulative scalar distances traveled by each atom were computed at each time step, and the maximum was compared to a test quantity, $0.95\left[1-1 /\left(n_{l}+1\right)\right]\left(r_{l}-2.50\right)$ wara $n_{l}$ is the number of time steps the list has been used. The test quantity anticipated whether or not the next step would move an atom outside the list range. The phase diegrem for two-dimensional Lennard Jonesium was 
eslublished by Barker ${ }^{2 ?}$ using perturbation theory for the fluld phases and a self-consistent cell model for the sollo phase. Monte Carlu calculations by Abrahan 23 confirmed Barker's phase bourdaries. Extensive equation of state data were compiled by Heivlerson ${ }^{24}$ using Monte Carlo calculations. These data were used to compute the pressure and sound speed in the liquid as function of tempurature at densities near $p^{*}=p \sigma^{2} \sim 0.6$ (roughly midway between the critical and Ilquid triple point densitles). Because no datu for the surface tension were avallable, molecular dynemics was used lo calculate the size-dependent surface tension, as discussed in Secliun A.5.

The inltial conditions for 13 fragmentation runs are shown in Flg. A.2 and tabulated in Table A.1. The four system sizes (169, 721, 2611 and 14491 atoms) correspond to crystals in the shape of regular hexugural crystals with $8,16,30$ and 70 rows of particles on a side. These configurations were used to initiate the runs. (Each crystal was melted and equllibrated to approximately the desired temperature and density within a confining wall; the fragientations runs began when the wall was removed).

Verlet's algorithm, with a time step, $\Delta t$, of $\sim 0.02$ in units of $\left(n r^{2} / x\right)^{1 / 2}$, was used,

$$
\vec{r}_{+}=2 \vec{r}_{0}-\vec{r}_{-}+\vec{F}_{0}(\Delta t)^{2} / m
$$

where $\vec{r}_{-}, \vec{r}_{0}$, and $\vec{r}_{+}$are the atom locations at three succesive times, and $\vec{F}_{0}$ is the total force from all atoms within 2.50. Velocities mre obtained from the coordinates, 
F1g. A.2. Initial zonditions for the 13 molecular dynemics fragmentation runs.

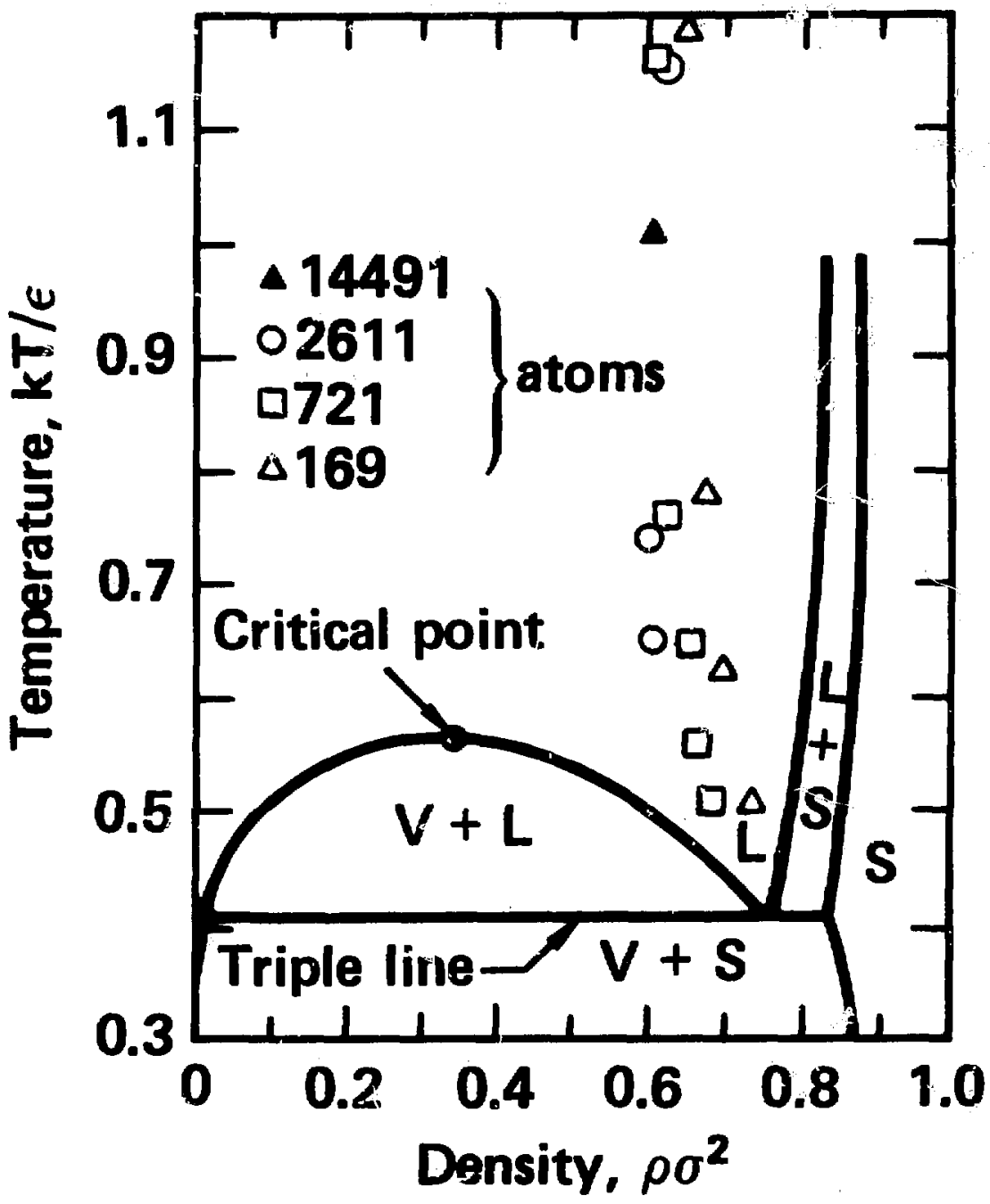


Tole A.1. Inltinl andilems on realts frem the two-diensional Dleceiter ameles runs. The cluster papulotions are Indicated by $N_{c}$ in is the ment of menemars; end $N$ is the orlolinal system mouletion:

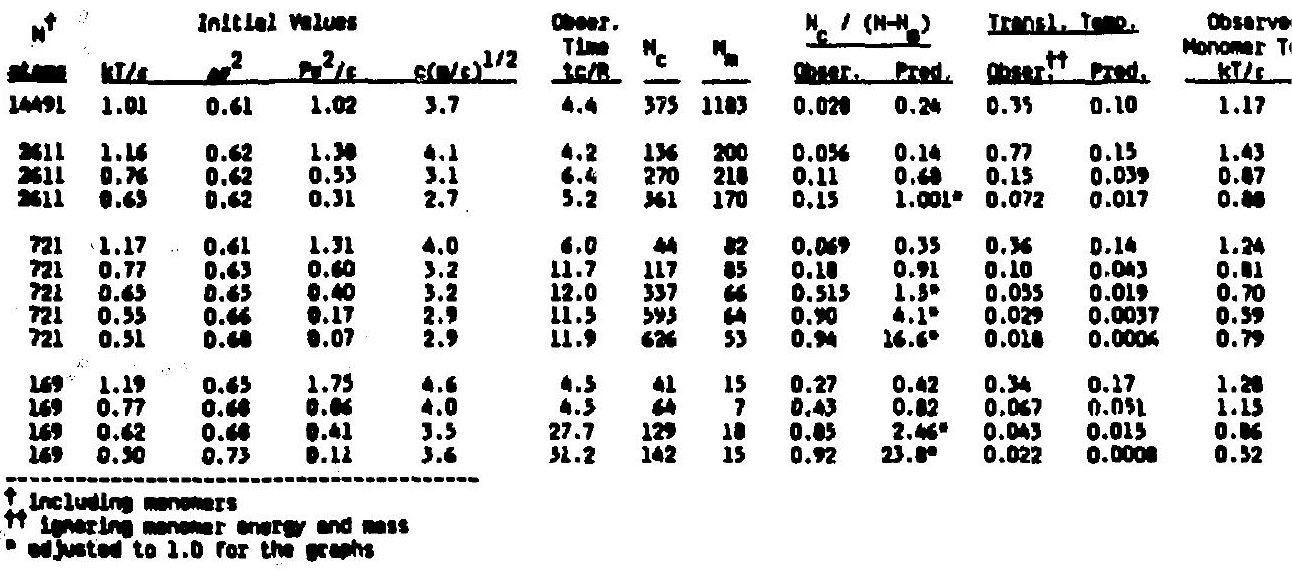




$$
\vec{v}_{0}=\left(\vec{r}_{+}-\vec{r}_{-}\right) / 2 \Delta t
$$

The pulcntlal, translational, rotational and thermal energies and the fragment sizes were tabulated. Fragment translational timporatures were defined as the fragments' center-of-mass kinetic energles in the fixed frame of reference divided by the the product of Boltzman's constant, $k$, and the corresponding fragment's size (in atoms). Fragment rotational temperatures were dafined is $L^{2} / K_{c} I$ whare $L$ is the angular momentum around the fragient center and I is the moment of inertia. Fragment thermal temperatures were given by remaining kinetic energy divided by the product of $k$ and the corresponding fragnent's size.

\section{A.5. Results}

\section{A.5.A. Imo Dimensional surface Tension}

Recenlly, Thompson, et. al. ${ }^{29}$ published an extensive molecular dynamics study of three dimensional surface tension that used time averuges of up to $t^{*}=t\left(\mathrm{mo}^{2} / \varepsilon\right)^{1 / 2}=1400$. To extend Thompson's study to two dimensions, a series of molecular dynamics calculations was performed on systems ranging from 70 to 700 atoms at reduced temperatures $\left(T^{*}=k T / \varepsilon\right)$ below, near, and above the triple point. Density and (Irving-KIrkwood) pressure tensar tine averages were laken for up to $t^{*}=1450$ in a few cases, with typical averaging periods being for $t^{*}=400$ to 700 . Analysis of the results indicates that the runs below the triple point did not reach equilibrium. (Low densilies and regative pressures typical of the two-phase solio-vepor region were measured.) For the higher temporature runs (T* $=k T / \varepsilon$ 
$\sim 0.45)$, fluctuations and occasional drop fragmentation obscured the results. Only near the triple point were reasonable time averaged pressure tensor profiles obtained.

The surface tension measured at the proper radius, $R_{s}$ (which is significently different then the equimoler radius for small drops), is

$$
r=\int_{0}^{\infty}\left(P_{N}-P_{T}\right)\left(\frac{T}{R_{S}}\right)^{D-1} d r
$$

where $D$ is again the dimensionality ( 2 or 3 ), $P_{N}$ and $P_{T}$ are the normal and tengential components of the pressure tensor, and the zero of I is within the bulk of the fluid (where $P_{N}=P_{T}$ ). The integrand is non-zero only near the interface. The normal and tangential components are related by a force balance, leading to

$$
P_{N}-P_{T}=-\left(\frac{T}{D-1}\right)\left(\frac{\partial P_{N}}{\partial T}\right)
$$

In 2 or 3 dimensions. Finally, the Laplace equation is used to elininate $R_{\mathbf{s}^{\prime}}$

$$
\Delta P=P_{N}(\text { liquid })-P_{N}(\text { vapor })=\frac{(D-1) Y}{R_{S}},
$$

The result is

$$
r=-\left[\frac{\Delta P^{D-1}}{(D-1)^{D}}\right] \int_{0}^{\infty} \frac{\partial P_{N}}{\partial r} r d r
$$

in 2 or 3 dimensions. The resulting surface tension at $T^{*}=0.35$ is plotted in Flg. A.3 as a function of $1 / M_{2}$, where $M_{2}$ is the number of liquid atoms in the drop. For interpolation, we assume that the surfece tension decreases linearly with temperature to zero at the 
Fig. A.3. Two dimensional surface tension at $k T / c=0.35$ from molecular dynamics calculations.

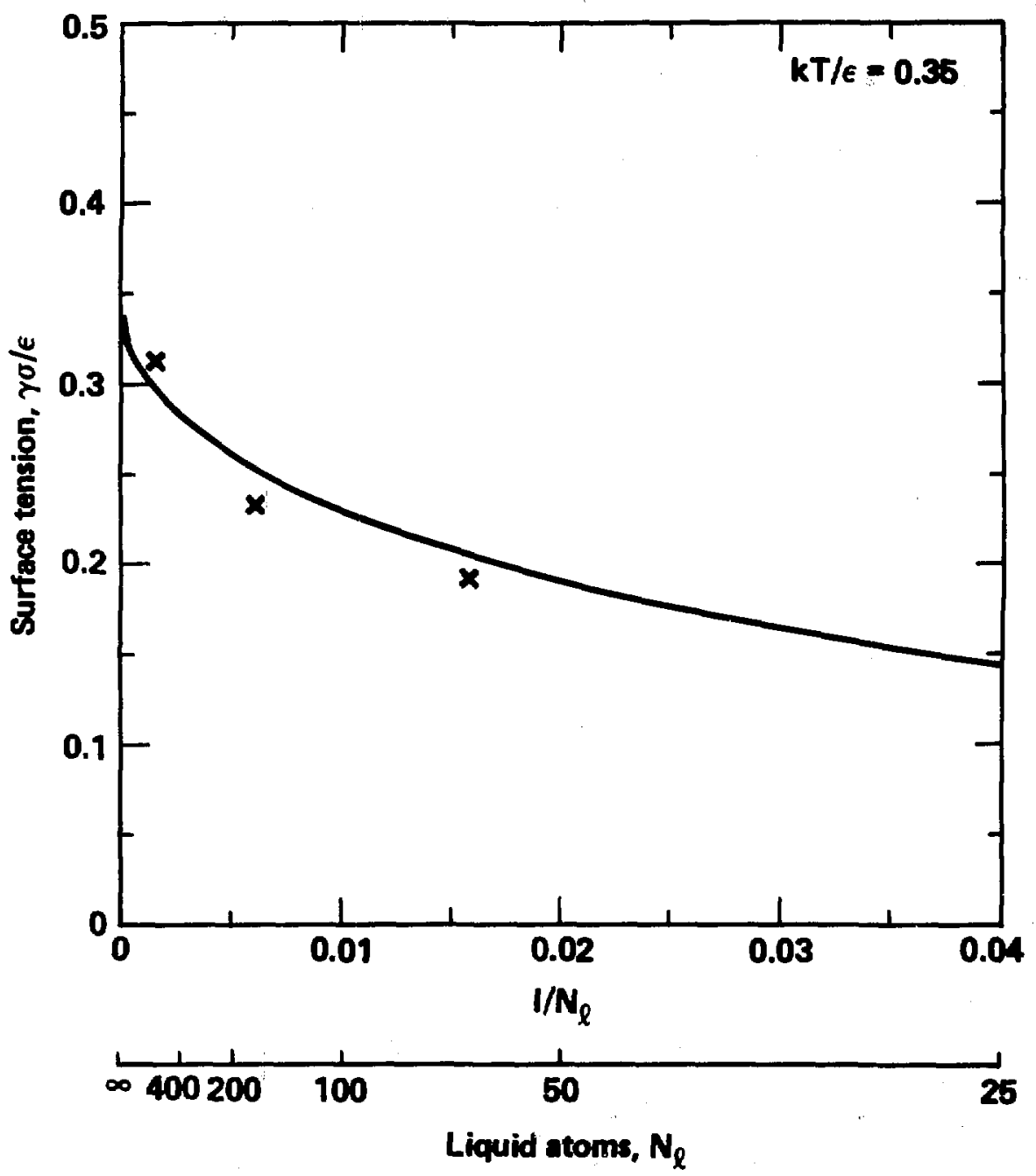


critical point, and that it also falls to zero for a single atom system,

$$
\gamma=0.35\left[1-\left(N_{l}\right)^{-1 / 2}\right]^{4}\left[1-\left(\frac{T^{*}-0.35}{0.21}\right)\right](\varepsilon / 0) .
$$

A simple scaling model based on excess energy due to missing bonds for surfuce atoms produced reasonable agreament between the two-tlimensional data and Thompson's three-dimensional results.

\section{A.5.B. Ernoment Slze as a Function of System S1ze and Pressure}

Frument sizes were usually tabulated after only a few sound traversal times in order to minimize the influence of vapor pressure formalion (Table A.1). In cases where fragmentation was marginal, much more tine elapsed before fragmentation. Examination of the atomic position plots for such cases indicates that some reglons osclllated (changed overall shape) several tlmes before fragmenting.

The fragment sizes (in atoms) from two 2611 atom runs are shown in Fig. A.4. The largest fragnent, the average of the ten largest fragments, and the average of the largest fragments conprising $30 \%$ of the iwn-monomer population are shown. In each case, the raw data have baen slightly adjusted (using the energy minimization model, "model 3") to unlform initial conditions (2611 non-monomer atoms, $p^{*}=0.6, c^{*}=c(n / c)^{1 / 2}=4.0$, and $\left.\gamma^{*}=0.32\right)$. The only remaining varieble in modal 3 is the initial pressure. The slopes of the three frowint size curvas are $-1.03,-0.86$, end -1.42 , respectively; these values bracket the predicted slope of $-4 / 3$ from model 3 reasonably mil. To 1llustrate the sensitivity of the fragmentation to 
Fig. A.4. Observed and predicted fragment sizes (atoms) as a function of Initial pressure, for two 2611 atom runs. The ota and model predictions have been slightly adjusted to constant initial conditions. The model numbers refer to Eqs. A.1 through A.4. The three observed values for each run are for the largest fragment, the average of the ten largest fragments, and the average of the largest fragments conprising $30 \%$ of the system population (less monomers).

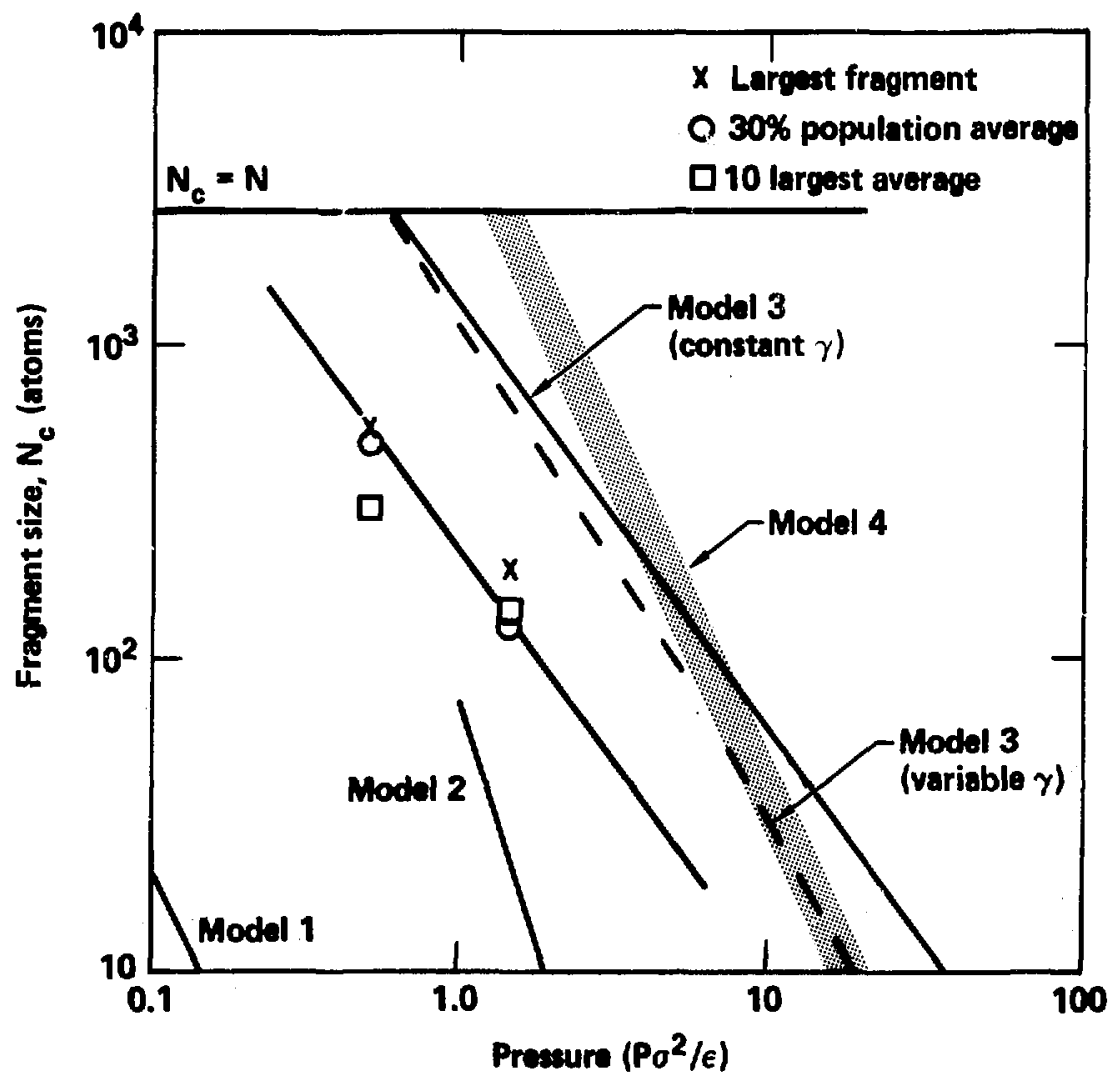


fragment-size dependent surface tension, the prediction curve is also showl using the lower surface tensions at the actual fragment sizes; the "observed" curves would be simflarly steeper when the surface tension correction (to $\gamma^{*}=0.32$ ) is elininated. Finally, the other model predictiens are shown. The slopes of models 1,2 , and $4(-2$, -4 , and -2 , respect $(k e i y)$ are much steeper then the molecular dynamics results.

The fragnent sizes from the highest pressure run for each system size (14491, 2611, 721, and 169 atoms) are shown in Fig. A.5. The nomernilature is identical to the previous figure. As in Fig. A.4, the data wre slightly adjusted to uniform initlal condit lons $\left(P^{*}=\right.$ $P \sigma^{2} / c=1.4, p^{*}=0.6, c^{*}=4.0$, and $\left.\gamma^{*}=0.32\right)$. The only remulining variable in the models is the system size (total number of parlicles), $N$. The slopes of the mlddle section of the three fragment slze curves are $0.75,0.69$, and 0.64 , respectively. These observed values bracket the predicted slope of $2 / 3$ from model 3 . The 169 atom data were not used to compute the slope because of minimal fracmentation. The 14491 atom fragnent sizes are probably a bit low. Posilion snapshots and density profiles Indicate that a sloshing molion was present in the systen at the time the confining boundary was rumoved. However, If the slopes are computed using line segments between the 721 and 14491 data, the results are $0.54,0.50$, and 0.41 , respexclively. Thus, the molecular dynanics results indicate a possible system stze dependence of the 2/3 power (the illustrated line), but support that slope with en uncertainty of \pm 0.15 . This result equin is consistent with the energy minimization model (model 3). Models 1 and 2 include no system size dependence and accordingly 
Fig. A.5. Observed and predicted fregment sizes (atom) as a function of system size, for simllar heating levels. The nomenclature is Identical to Flg. A.4.

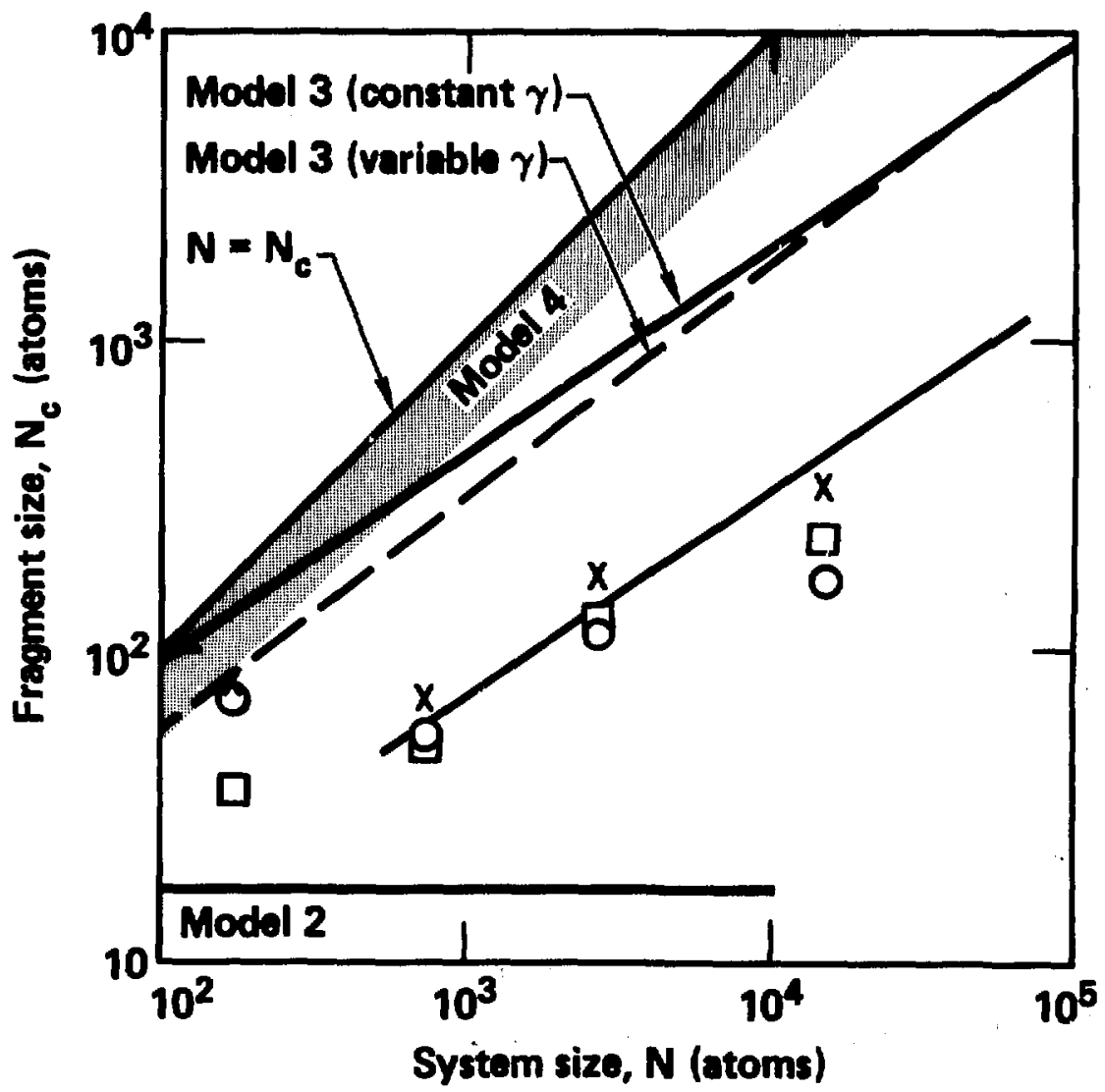


prodict very low fragment sizes for this initial condition 0.1 and 18 atoms, respactively). Model 4 predicts a much steeper slope (1.0) then found in the molecular dynamics runs.

Although the data in Figs. A.4 and A.5 support the energy minimization model's power law dependence of fragment size on system sizo and lsochoric heating level (initlal pressure), the magnitudes of the ubserved sizes (In atoms) are about ten times lower than the model 3 prediction. we therefore suggest that the predictions of that model be raduced tenfold in applications.

\section{A.5.C. Time Evolution of the System}

As time evolved in the thirteen numerical experiments, snapshots of the atomic positions were used to visualize the fragmentation; a semple sequence is shown In Fig. A.6. Inspection of this figure indicules that the average fragment size is smallest near the system center, in agreenent with model 3 when the variation of velocity diveruence with radius is considered.

A more quantitative description of the fragmentation process is contained in Table A.I and in Figs. A.7 and A.B which show the trwislational and thermal temperatures and the largost cluster size as funclions of tim, system population and initlal temperature. The abselsse of each plot, tc/R, is the time divided by the sound truversal time. The Initial temperature corresponds to the $y$-intercepts in Fig. A.7. To decrease the influence of vapor pressure formation on the results, both thernal and translational temperatures are based on the total mumber of non-monomer atoms in the system. The rotational temperature was not plotted because it was always 
F1g. A.6. Snapshots of the atomic configurations at four tims diring the fragnentation process of 14491 atom system $(k T / c \sim 1.0)$.

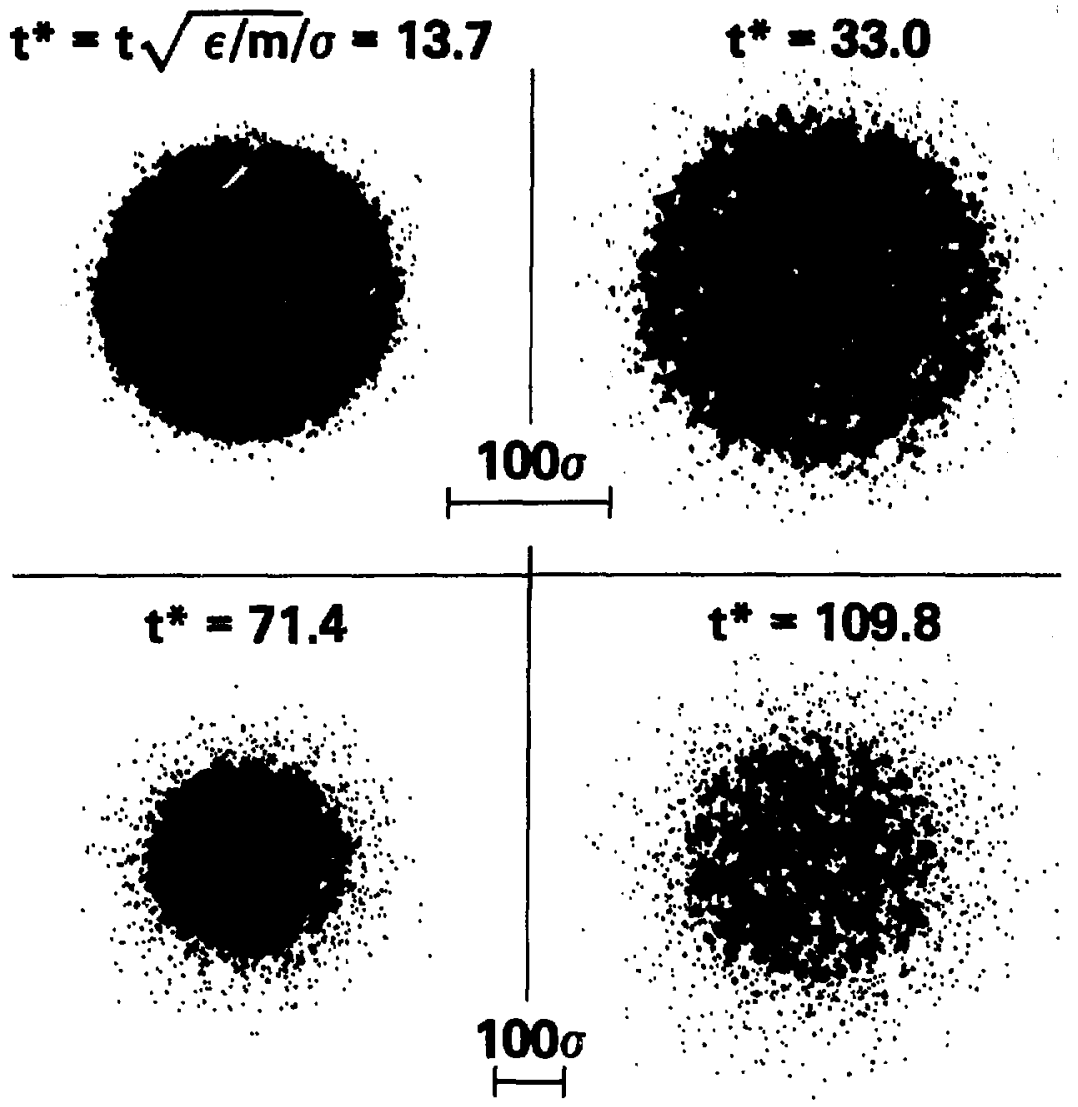


Fig. A.7. Thermal and translational temperature evolution in the fragmentation runs.

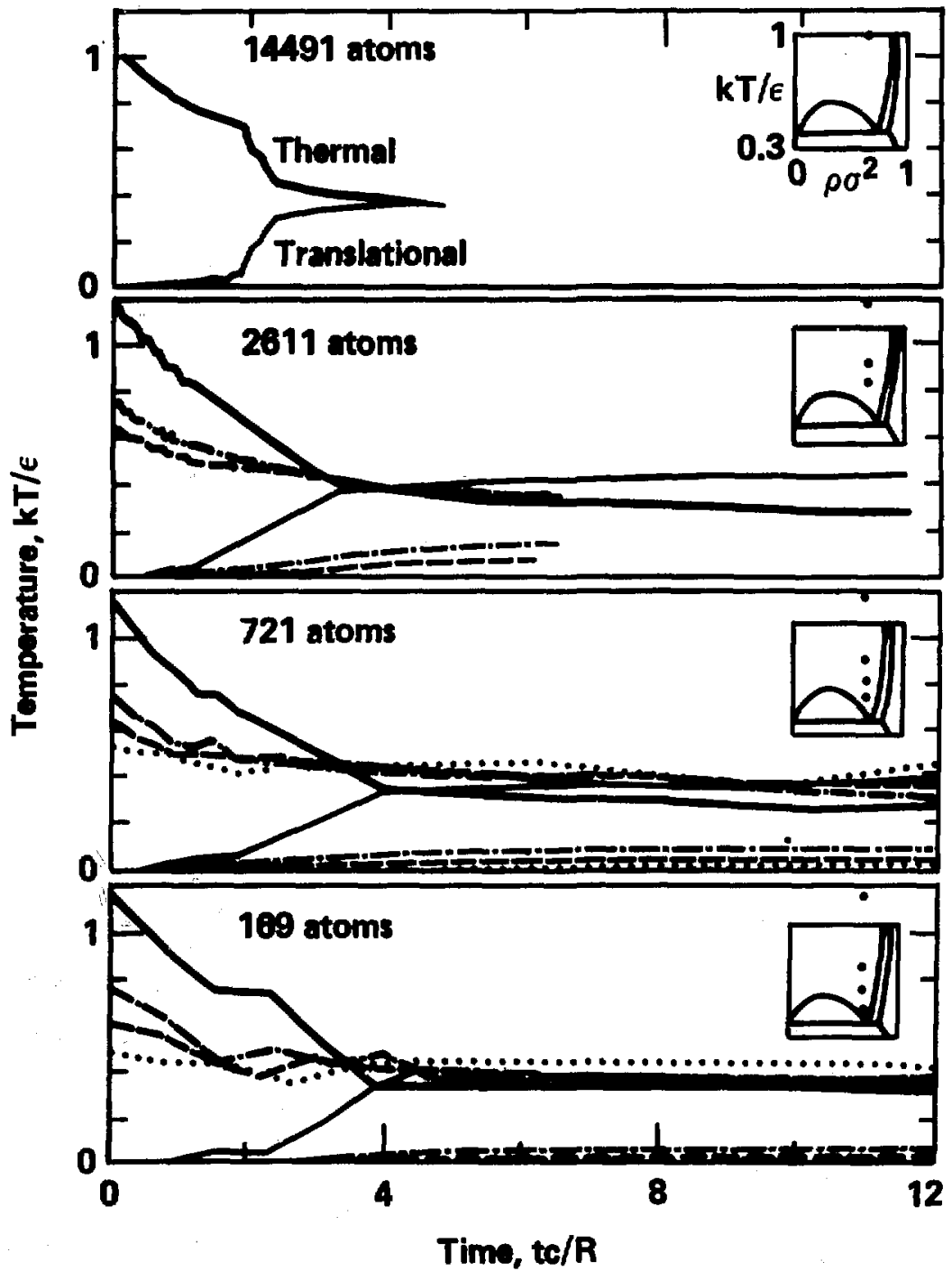


F1g. A.B. S1ze of the largest cluster as a function of time in the 2-D fragmentation runs. The ordinate is scaled according to modit 3 to ellminate the system size dependence.

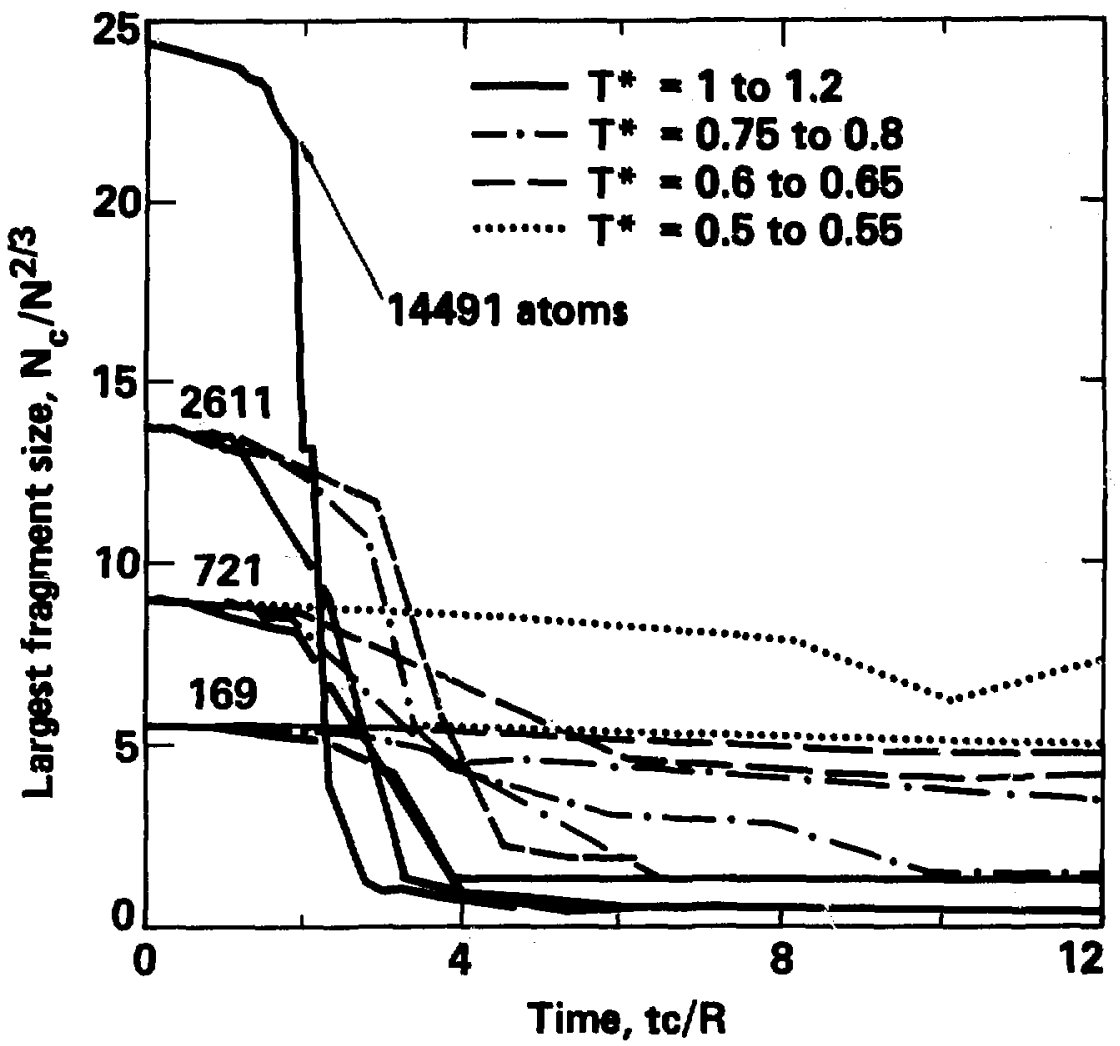


nogligibly small.

The thermal temperature typically relaxes to a "post-fragmentation" level of about 0.35 to 0.4 (near the triple point) after two or three pressure relief times (R/C) (Fig. A.7). The translational temperature reaches its "post-fragmentation" value at about the same tine, but this value depends on the initial temperature. For the highest initlal temperature lovel $\left(T^{*} \sim 1.15\right.$ ), the final trenslationel temperature was $\sim 0.4$, somewhat higher than the finel thermal temperature. For the lower Initial temperatures, the final translational temperature was 0.1 or lower. As predicted by model 3, there is an observable system size dependence for the translational temperature, with the larger systems attaining higher values. The total of the final translational and thermal temperatures was always less than the initial thermal temperature; the remaining energy was elther carried as kinetlc energy by monomer atoms in the vepor, or stored as potential energy in the fragments. The system potential energy increases due to bond breaking during vaporization and surface area growth (fragnentation), and decreases slightly due to the increase in bulk density caused by the lower liquid temperature. The evolution of the largest cluster size is shown in Fig. A.8. The ordinate is scaied by $1 / N^{2 / 3}$ to remove the system size dependunce predicted by model 3 . When it was widespread, frequeritation typically occured at about two to four sound traversal tIns. The $T^{*} \sim 0.75$ runs appear to be borderline for the three smllest system sizes, with fragmentation occurring in steps over an extended period of time much longer than a few sound traversal times. The paths follownd on T-p diagrem are shown in Fig. A.9 for 
Fig. A.9. Pressure-density paths followed by two fragmentation run's. The densitles were calculated in an average sense; actual local density in the fluid did not reach such low values.

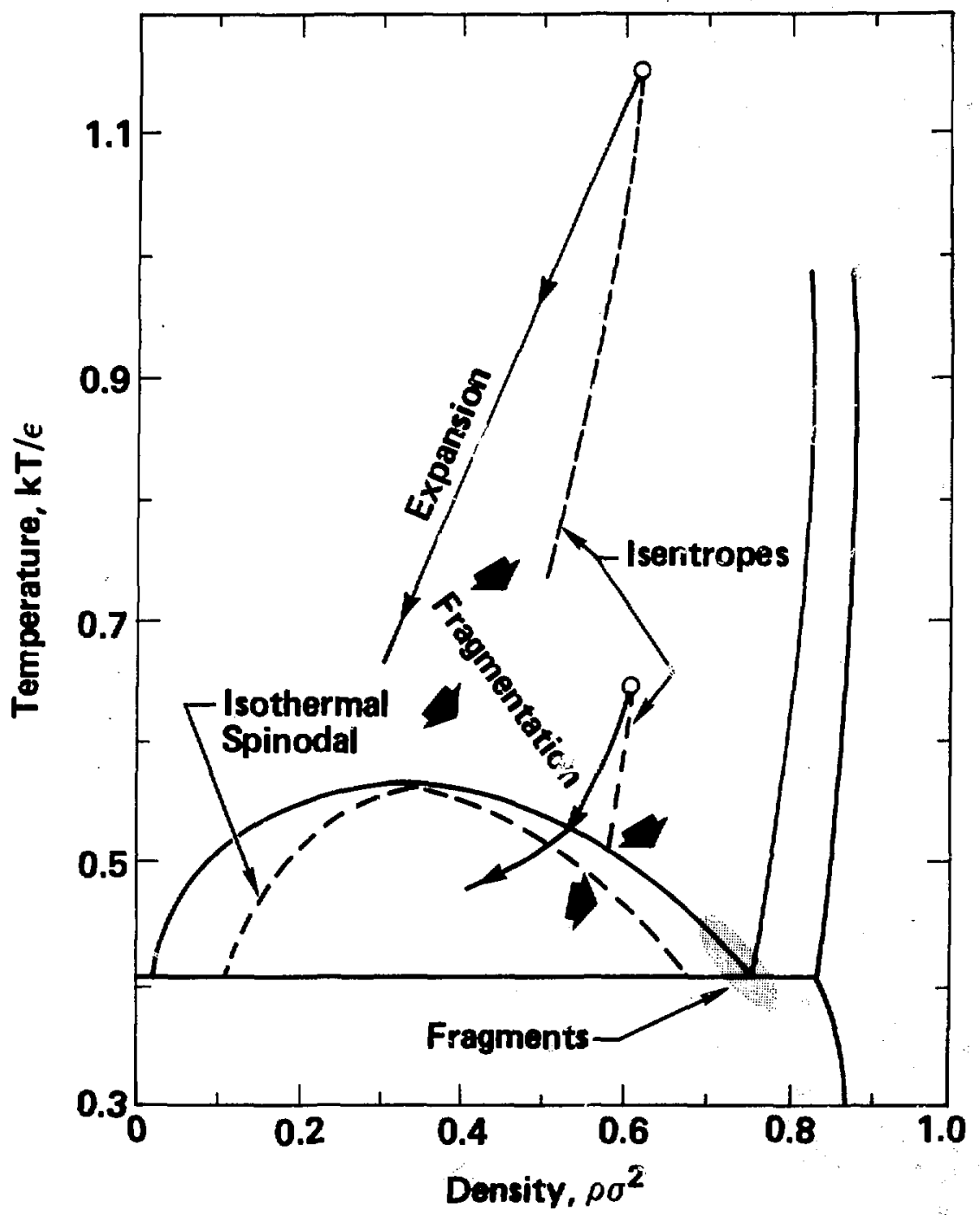


two 2611 atom runs. The paths shown use spatially averaged densities Up to the fregwentation point, but inspection of the position plots shows marked haterogeneity much earlier. (The definition of frequentation used here requires a minimum fragment separation of 2.5..) Internal voids which eventually become fragment surfaces eppear much tearlier. The internel volds decrease the "pre-fragment" densily; hence, density appears to increase upon fragnentation. Temperature also decreases; as a new daughter fragnent is born, its "center-of-mass translational energy" is instantaneously subtracted from the "internal" energy of Its parent cluster. Most of the fragment temperatures 110 along the saturated liquid phase line. Finally, although the low temperature run appeared to penetrate the unstable region under the 1sothermal spinodal (and may have reached the isentrcpic spinodal which lies below the isothermal spinodal), the regular density patterns ${ }^{64,65}$ characterlstic of "spinodal docomposition" ware not observed. This is because the fragmentation expunsion is inhomogeneous. If the internal voids that precede fragmentation were not included in the density calculation, then the spinodal lines would not be reached.

\section{A.S.D. Frequant Size Distribution}

The integrated (cumulative) population distributions for three runs with sinilar heating levels and varying system slzes are show in F1g. A.10. The smallest (721 atom) system had a sionlficent portion of the population concentrated in en intermediate fragment size range (indlated by the steep portion of the curve). The larger systems' 
Fig. A.10. Integrated population distributions as a function of fragment size for three fragmentation runs with sinilar heating levels but different system slzes. The dashed lines give integrals as functions of the fractional fragient size, $N_{c} / N$, and the solld lines are normalized to the largest fragment size, 1.e. the abscissa is $\mathrm{N}_{\mathrm{c}} / \mathrm{N}_{\mathrm{c}} \max$. The fragment data were taken just as fragment sizes becume well defined.

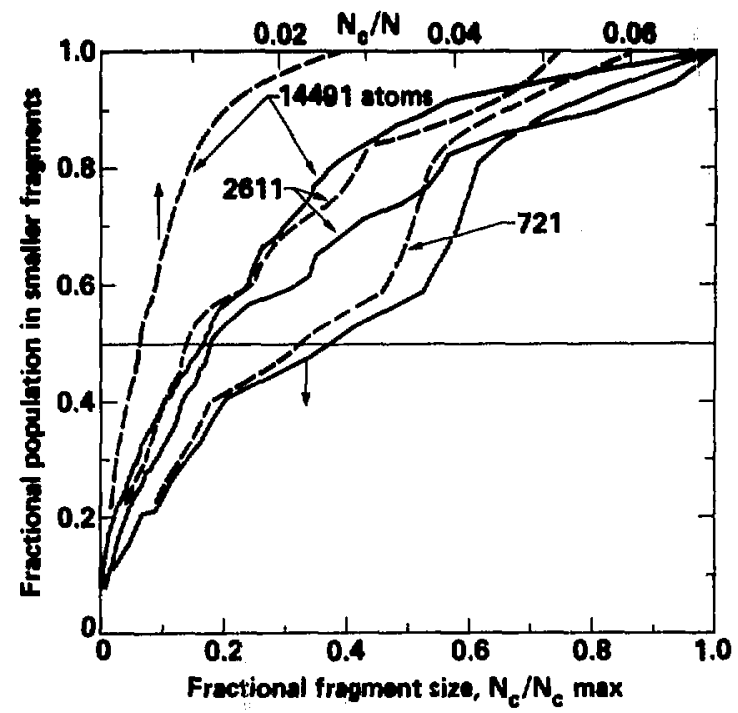


exhlbited decreasing population density with increasing fragnent size. The overall effect of increasing system size was to concentrate population in smiler frectional fragment sizes; 50\% of the population we in fregments smaller then $36 \%, 18 \%$, and $17 \%$ of the largest fragment size for the 721,2611 , and 14491 atom systems, respectively.

\section{A.5.E. Irensintional Eneroy of the Froments and Monomars}

The trenslational temporatures (Ignoring nonomor energy and mass) are shown in Fig. A.1l as function of system size and inltial pressure. The Inset shows tha observed trenslational tomperatures which properly increase with hating level (Initial pressure). All the values exceed $m v^{2} / 2 k$, where $v$ is the jump-off velocity, (dashed line). In two dimensions, modol 3 prodicts

$$
T_{t r}=\left(\frac{r^{2}}{2 k}\right)\left[1-\frac{1}{2} \frac{N_{c}}{N}\right]
$$

For extreme fraguentation $\left(N_{c} \rightarrow 0\right)$, Eq. A.13 produces the correct uppar bound, but for no fragwentation Eq. A.13 predicts 1/2 the upper bound, rachor then zero. The failure at no-fragwentation is a consequence of the assumption of a homogeneous expansion in model 3 .

The min portion of the figure shows the ratio of the observed to the predicted (Eq. A.13) trenslational temperatures. The additional kinatic enoroy is from two sources: vepor pressure formation (which is IInited in Fig. A.11 by the elimination of monomers from the calculation), and fragantation dus to fluctuations (a phenomenon chearved for sall systens on the saturation line with no isochoric heating). It is also possible that additional kinetic energy is 
Fig. A.11. Translational temperatures (scaled to the prediction of model 3) for the 13 molecular dynamics runs.

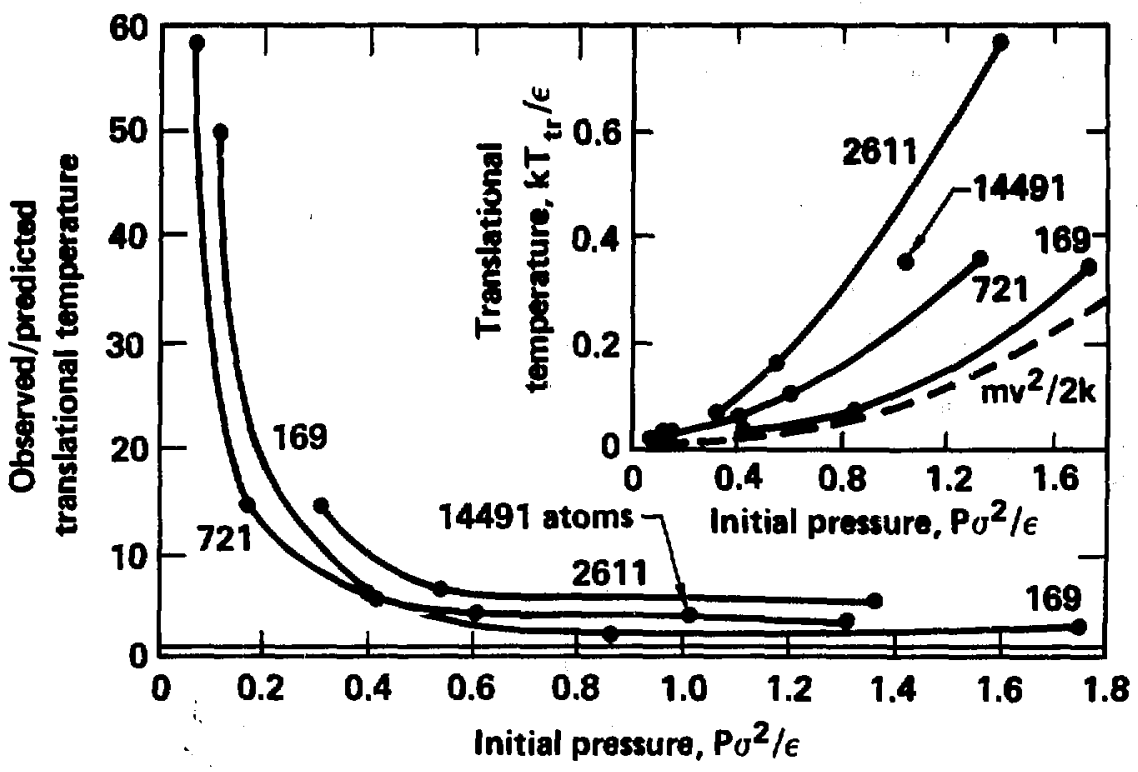


produced by the recoll of the stretched material when it fragments.

For macroscopic systens, model 3 suggests that both vapor pressure formalion and fracture due to "equilibrium" fluctuations are negligible. Glem's three dimensional (macroscopic) hydrodynamic code calculations ${ }^{46}$ confirmed the upper bound of mpv ${ }^{2} / 2 k$ wten no tensiun is allowed in the materlal. Our molecular dynanics calculations (F1g. A.11) show that the trenslational temperature tends to Increase with system size, at least for systems of a few thousand atoms or less. However, the 14491 aton result shows a decreased trenslational temperature with increasing system size (even though its initial sloshing motion would be expected to produce more trensiational ervergy than an initially quiescent system). Thus, these linited molecular dynemics results are not inconsistent with model 3. Finally, we note that the peak in the excess translational temperature is in the region of the critical nucleus size. Systems smaller than the critical size are naturally unstable, leading to fragmentation even in saturated systens.

The monomar temperatures at the time of fragmentation for each run are listed in the last column of Table A.l. The average liquid temperature during the monomer formation period lies somewhere between the pack listed in the second colum of the table and the saturation temperature $(\sim 0.5)$. The monomer temperature exceeds both the everuge and the peak liquid temperatures in every case. Similar results have been found by Vicentinl, Jacucci and Pantharipande 63 using three dimensional molecular dynamics for systens of 250 atoms. Vicentini, at. al. also found that the thermal temperatures of most of the fregents are similar and essentially indspendent of the initiel 
heating level, in agreement with our results.

\section{A.6. Conclusions}

Two dimensional nolecular dynamics fregmentation simulations were run using five levels of heating and four system sizes. The numerical experiments produced agreement with only one of four proposed models (the energy minimization nodel, model 3). The nodel's power law dependence of fragment size (atoms) on system size (2/3) and initlal pressure $(-4 / 3)$ were both supported by the data. The larger fragmant sizes were about an order of magnitude smaller than the model 3 predictions. There was an unexpectedly large level of translational energy generated In these molecular dynemlcs runs, probably due to the combination of small system size (fluctuations) and near-critical saturation temperature (vaporization). In macroscopic systams, model 3 (with the 0.1 correction factor) predicts

$$
r=\left[\frac{D+2}{p} Y\left(\frac{R c}{\Gamma e}\right)^{2}\right]^{1 / 3} 10^{1 / 0}
$$

where $r$ is the fragment radius, $R$ is the system radius, $D$ is the number of dimensions ( 2 or 3 ), $\Gamma$ is the Gruneisen parameter, $e$ is the added heat per unit mass, and $p_{m}$ is the mass density. When Eq. A.14 is applied to the HYLIFE reactor initial conditions $0.1 \mathrm{~m}$ redius lithium jets, $800 \mathrm{~kJ} / \mathrm{kg}$ added energy, $485 \mathrm{~kg} / \mathrm{m}^{3}$ density, 1.0 Grüneisen paraneter, $0.35 \mathrm{~J} / \mathrm{m}^{2}$ surface tension, and $4500 \mathrm{~m} / \mathrm{s}$ sound speed, the predicted fragment radius is $2.1 \mathrm{~mm}$. Thus, fragmentation wlll produce about two orders of magnitude increase in the surface area, making it plausible that the required pulse rate of $1.5 \mathrm{~Hz}$ can be achieved. The molecular dynamics fragnent sizes yary as a powar of 
system size which could be lower than $2 / 3$, perhaps as low as $2 / 2$. Extrapolation of our results over 13 orders of magnitude in system size (Lo the number of atoms in a HYLIFE fet's cross section) gives a decrease in the predicted fragment cadius by about a factor of 25 . This veviation from model 3 is in the favorable direction from a reactor designer's point of view. 
James Allen Blink was born on Aprll 21, 1948 in Kenosha, Wisconsin. He attended elementary school in Kenosha and funlor. high school in Tucson, Arlizona. In 1966, he graduated as valedictorian of his class at Basic High School in Henderson, Nevada. In 1970, he graduated "With Distinction" from the University of Navada at Reno. In addition to hls Bachelor of Sclence degree in Englneering Science, Jim was comnissioned a Second Lieutenant in the United States Army Corps of Englneers. After spending the summer working as a nuclear englneer at the Nevada Test Site, Jim began his first Army assignment: attending graduate school at the Georgla Inst1tute of Technology under the Army's "Top 5\%" program. In Septenber 1971, he graduated as a Master of Science in Nuclear Engineering. From 1976 to 1978, Jim was a military research associate in the Energy and Military Application Group of Lawrence Livermore National Laboratory's Inertial Confinement Fusion Program. During this perlod, he also earned his license as a Professional Englneer in the Commonwealth of Virglnia. In 1979, he left the Army and rejoined LLNL as a civilian employee. In 1980, Jim enrolled in the University of California at Davis/Livermore to complete his PhD degree through the Department of Applied Science. 